University of Rhode Island

DigitalCommons@URI

Open Access Dissertations

2014

\title{
Lattice Boltzmann Simulation of Multiphase Flows; Application to Wave Breaking and Sea Spray Generation
}

Amir Banari

University of Rhode Island, amir_banari@my.uri.edu

Follow this and additional works at: https://digitalcommons.uri.edu/oa_diss

\section{Recommended Citation}

Banari, Amir, "Lattice Boltzmann Simulation of Multiphase Flows; Application to Wave Breaking and Sea Spray Generation" (2014). Open Access Dissertations. Paper 282.

https://digitalcommons.uri.edu/oa_diss/282

This Dissertation is brought to you for free and open access by DigitalCommons@URI. It has been accepted for inclusion in Open Access Dissertations by an authorized administrator of DigitalCommons@URI. For more information, please contact digitalcommons-group@uri.edu. 
LATTICE BOLTZMANN SIMULATION OF MULTIPHASE FLOWS; APPLICATION TO WAVE BREAKING AND SEA SPRAY GENERATION BY

AMIR BANARI

A DISSERTATION SUBMITTED IN PARTIAL FULFILLMENT OF THE REQUIREMENTS FOR THE DEGREE OF DOCTOR OF PHILOSOPHY

IN

OCEAN ENGINEERING 
DOCTOR OF PHILOSOPHY DISSERTATION

$\mathrm{OF}$

AMIR BANARI

APPROVED:

Dissertation Committee:

Major Professor Stephan T. Grilli

Tetsu Hara

Jason Dahl

Nasser H. Zawia

DEAN OF THE GRADUATE SCHOOL

\section{UNIVERSITY OF RHODE ISLAND}

2014 


\begin{abstract}
Simulating complex fluid flow have always been one the most challenging problem in Computational Fluid Dynamics (CFD). Most of these difficulties come from the deficiencies of Classical CFD method in computational time and boundary implementations. Recently the Lattice Boltzmann Method (LBM) has been recognized as an alternative to the classical CFD methods for its advantages such as , easy boundary implementation, suitability for parallel simulation and no need for the Poisson pressure solver.

The LBM is based on the Boltzmann equation, which governs the dynamics of molecular probability distribution functions, from a microscopic scale point of view. The primary variable of the Boltzmann equation is the particle distribution function $f(\boldsymbol{x}, t, \boldsymbol{\xi})$ which describes the probability to find a particle with microscopic velocity $\boldsymbol{\xi}$ at point $\boldsymbol{x}$ and time t. The macroscopic fluid properties (velocities, pressure) are computed as moments of these particle distribution functions.

This dissertation investigates LBM for complex multiphase fluid simulation in three manuscripts. In the first manuscript a novel LBM scheme is developed for simulating multiphase flow simulation with high density ratio. In previous multiphase flow simulations the maximum fluids density ratio achievable in computations was limited by the occurrence of instabilities for high density ratio values (typically larger than 10-20). Overcoming this limitation is one of the most challenging current issues in the LBM modeling of multiphase flows and the subject of the first manuscripts, as we aim at modeling complex flows at an air-water interface, whose density ratio is about 1,000 .

It should be pointed out that although the numerical scheme in manuscript one is able to simulate large density ratio multiphase flow for moderate Reynolds numbers it is not stable for high Reynolds numbers. Eliminating this deficiency
\end{abstract}


of the first manuscript for simulating of multiphase flows with high density ratios and high Reynolds numbers is the subject of second manuscript. In the second manuscript a novel LBM method is introduced which is able to simulate multiphase flow with arbitrary Reynolds and density ratios. The resulting algorithm is applied to several test cases, such as droplet splash, rising bubble and wave braking. The good agreement between numerical results and existing data demonstrate that the newly developed model is a useful tool for simulating complex multiphase flows.

In the third manuscript we study the effects of point-wise particles on turbulent channel flow. Investigating particle-laden turbulent flows is an important fluid mechanics problem as it occurs frequently in nature. This work has been done in collaboration with Professor Tetsu Hara and Yackar Mauzole in Graduate School of Oceanography at the University of Rhode Island to study the sea spray generation effects of turbulent layer above see surface in high wind condition. In this work the Lattice Boltzmann method is coupled to a Lagrangian particle tracking approach for simulating a system of fluid-particle flow.

In all three manuscripts the numerical schemes are developed in nVIDIA CUDA framework, which made it possible to efficiency implemented on General Purpose Graphical Processing Unit (GPGPU) to numerously increase the computational speed of the numerical code. 


\section{ACKNOWLEDGMENTS}

I would like to express my gratitude to my adviser, Professor Grilli, for his support, guidance and more importantly for trusting me for doing this research. Without his supervision and constant help this dissertation would not have been possible.

I also would like to thank my committee members, Professor Hara, Professor Dahl, Professor Licht and Professor Donohue. I specially want to thanks professor Hara for his great advice during this research.

I would like to express the deepest appreciation to Gail Paolino, for her kindness, for her help whenever I needed it and for being the best secretary ever.

I thank my office-mates and friends, Reza Aghajani, Kevin Hopkins, Christian Janßen, Sean Davis, John Montgomery, Sina Hamian, Teyebeh Tajalli Bakhsh, Alesandra, Morales, Yaurel Guadalupe Torres, Tania Lado, Ivan Blanco Rubio, Telli Davoodi, Akbar Bagri, Sara Kadkhodaie, Asie Ahani, Hossein Saheb Ekhtiari, Maryam Rouhi, Golnaz Rouhi, Arash Bigdeli, Amir Shirazi, Arash Shirazi, Amin Mivehchi, Mohsen Zayernouri, Jeff Harris and others for making these four years easier and enjoyable.

This study would not have been possible without the contribution and funding

of the National Science Foundation (OCE-09-27014 Physical Oceanography Program, the University of Rhode Island).

And Finally a special thanks to my family. Words cannot express how grateful I am to my parents, for their great kindness, their support and for believing in me. I also would like to thanks my brothers and sisters, Reza, Sara, Nasir and Asal for their love and encouragement. 


\section{PREFACE}

This dissertation is organized in manuscript format and is comprised of three main manuscripts focusing on three different topics. The first manuscript published in Computer and Fluids 93 (2014) 1-17. The second manuscripts has been submitted to Computers and Mathematics with Applications and is under review. The third manuscript will be submitted to Journal Computers and Mathematics

with Applications. This dissertation is followed by three appendices which provide additional details about proof of the equations and the numerical schemes. 


\section{TABLE OF CONTENTS}

ABSTRACT ............................ ii

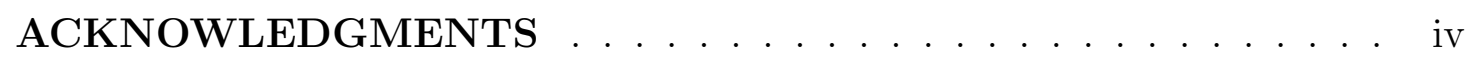

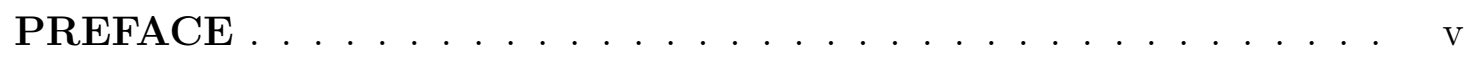

TABLE OF CONTENTS ................. vi

HIST OF FIGURES . . . . . . . . . . .

LIST OF TABLES $\ldots \ldots \ldots \ldots \ldots \ldots \ldots \ldots \ldots \ldots \ldots \ldots \ldots$ xvii

\section{CHAPTER}

1 Efficient GPGPU implementation of a lattice Boltzmann model for multiphase flows with high density ratios . . . . . 1

1.1 Introduction . . . . . . . . . . . . . . . . . 3

1.2 Diffusive interface models. . . . . . . . . . . . . . . . . 8

1.3 Lattice Boltzmann Model . . . . . . . . . . . . . . . . . . . 11

$1.3 .1 \quad$ Lattice Bolzmann solution of momentum equation . . . . 13

1.3 .2 LBM for solving Cahn-Hilliard equation . . . . . . . 20

$1.3 .3 \quad$ Boundary conditions $\ldots \ldots \ldots \ldots \ldots \ldots$

1.3 .4 Computation of spatial derivatives . . . . . . . . . . 22

1.3 .5 Summary of LBM algorithm . . . . . . . . . . . . 23

1.4 GPGPU Implementation of the LBM code $\ldots \ldots \ldots \ldots$

1.4 .1 Topology and grid mapping $\ldots \ldots \ldots \ldots$

1.4 .2 Implementation details $\ldots \ldots \ldots \ldots \ldots$

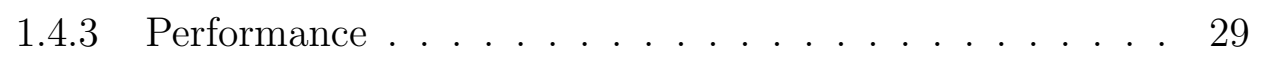

$1.5 \quad$ Applications . . . . . . . . . . . . . . . . . . . . 31

1.5 .1 Definitions . . . . . . . . . . . . . . . 32 


\section{Page}

1.5 .2 Two-fluid Poiseuille flow . . . . . . . . . . . . . . . . 33

1.5.3 Stationary bubble in quiescent fluid . . . . . . . . . . . . 37

1.5.4 Rising bubble in quiescent fluid . . . . . . . . . . . . . . 41

1.5.5 Rayleigh-Taylor instability . . . . . . . . . . . . . . . . 44

1.5 .6 Breaking wave . . . . . . . . . . . . . . . . . 45

1.6 Conclusions and outlook . . . . . . . . . . . . . . . . . 46

List of References . . . . . . . . . . . . . . . . . . . . . . . . . . . 51

2 An efficient lattice Boltzmann multiphase model for 3D flows with large density ratios at high Reynolds numbers . . . . . . 58

$2.1 \quad$ Introduction . . . . . . . . . . . . . . . . . . . . . . 59

2.2 Governing equations and numerical implementation . . . . . . . 64

2.2 .1 Lattice Boltzmann Methods . . . . . . . . . . . . . . . 64

2.2.2 Lattice Boltzmann solution of NS equations . . . . . . . 65

$2.2 .3 \quad$ Lattice Boltzmann scheme for interface capturing . . . . 68

$2.2 .4 \quad$ Numerical implementation and performance . . . . . . 71

2.3 Validation . . . . . . . . . . . . . . . . . . . . . . . 72

2.3 .1 Droplet impact on a thin wet surface . . . . . . . . . . . 74

2.3 .2 Rising bubble in quiescent fluid . . . . . . . . . . . . . 83

$2.3 .3 \quad$ Breaking wave . . . . . . . . . . . . . . . . . 88

2.4 Conclusions and Outlook . . . . . . . . . . . . . . . . . 100

2.5 Acknowledgment . . . . . . . . . . . . . . . . . 103

List of References . . . . . . . . . . . . . . . . . . . . . . . . 105

3 The simulation of turbulent particle-laden channel flows by the Lattice Boltzmann method] . . . . . . . . . . . . . . . . . . . 107 
$3.1 \quad$ Introduction $\ldots \ldots \ldots \ldots$

3.2 Direct Numerical Simulation of fluid flows with a Lattice Boltzmann Method . . . . . . . . . . . . . . . . . . . . . 112

$3.2 .1 \quad$ LBM governing equations $\ldots \ldots \ldots$

$3.2 .2 \quad$ Boundary condition on solid walls $\ldots \ldots \ldots \ldots \ldots$

$3.2 .3 \quad$ Definition of parameters for DNS of turbulent channel flows 117

$3.3 \quad$ Modeling of particle-laden flows $\ldots \ldots \ldots$. . . . . . . . 122

$3.3 .1 \quad$ Key physical concepts . . . . . . . . . . . . . . . 122

$3.3 .2 \quad$ Numerical algorithm for particle tracking and volume forces 126

3.4 Results . . . . . . . . . . . . . . . . . . . . . . . . . . . . . . . 129

3.4.1 Validation of turbulent channel flow simulations . . . . . 129

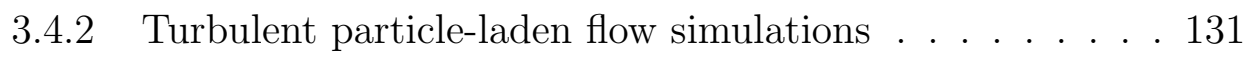

$3.4 .3 \quad$ Mean and r.m.s. flow velocity . . . . . . . . . . . . . 132

3.4 .4 Streamwise force balance . . . . . . . . . . . . . . . 134

$3.4 .5 \quad$ Production and dissipation of mean TKE . . . . . . . . 139

3.4 .6 Turbulent coherent structures . . . . . . . . . . . . . 141

$3.5 \quad$ Summary and conlusions $\ldots \ldots \ldots \ldots$. . . . . . . . 147

\section{APPENDIX}

A Chapman-Enskog expansion for pressure-less NS equations . 151

B Chapman-Enskog expansion for Cahn-Hilliard equation . . . 155

C Chapman-Enskog expansion for pressure Poisson equation . 157

D Chapman-Enskog expansion to LB equations for solving multiphase flow with high density ratio and high Re number flows to retrieve Navier Stokes equation $\ldots \ldots \ldots$ 
E Lattice Boltzmann method with non-uniform grid . . . . . . . 169 


\section{LIST OF FIGURES}

$1.1 \quad$ D2Q9 Lattice for definition of particle velocities . . . . . . . 13

1.2 Definition sketch of a two-fluid Poiseuille flow between infinite plates, with typical analytical solution for the horizontal velocity profile $u(y) \ldots \ldots \ldots \ldots \ldots \ldots \ldots \ldots \ldots$

$1.3 \quad$ Non-dimensional velocity profiles $u^{\prime}=u / u_{1}^{\max }$ in two-fluid Poiseuille flow (Fig. 1.2), for $\mathrm{Re}=100, \mathrm{Ma}=0.01, \rho_{1} / \rho_{2}=100$, and $\nu_{1} / \nu_{2}=0.1:(-)$ analytical; (o) LB results (only $33 \%$ of nodes are plotted for clarity), for different grid resolutions $h^{\prime}$ (see, Table 1.2$) \ldots \ldots \ldots \ldots \ldots \ldots \ldots \ldots$

1.4 $L_{2}$-norm error (between analytical and LB results) as a function of LBM discretization size $h^{\prime}$, for the two-fluid Poiseuille flow test case (Fig. 1.2): (•) data from Table 1.2; ( $\square$ ) power curve fit $L_{2} \propto\left(h^{\prime}\right)^{-1.008}\left(R^{2}=0.99\right) \ldots \ldots \ldots \ldots \ldots$

1.5 Two-fluid Poiseuille flow (Fig. 1.2). Non-dimensional velocity profiles $u^{\prime}=u / u_{1}^{\max }$ for $\mathrm{Re}=1000$ and $\mathrm{Ma}=0.005$ : $(-)$ analytical; (o) LB results for $N=300$ LBM nodes (only $33 \%$ of nodes are plotted for clarity). (a) $\rho_{1}^{\prime} / \rho_{2}^{\prime}=1$ and $\nu_{1} / \nu_{2}=0.1$, (b) $\rho_{1}^{\prime} / \rho_{2}^{\prime}=100$ and $\nu_{1} / \nu_{2}=1$, (c) $\rho_{1}^{\prime} / \rho_{2}^{\prime}=1000$ and $\nu_{1} / \nu_{2}=0.0667$. 39

1.6 Time evolution of the shape of an initially rectangular droplet towards a circle of radius $R^{\prime}=68\left(\rho_{1}^{\prime}=600, \rho_{1} / \rho_{2}=100\right)$, as a result of Laplace law; $t^{\prime}$ is non-dimensional time of computations (i.e., number of time steps). . . . . . . . . . . . . . . 41

1.7 Velocity vectors computed around the bubble interface at four different times equal or larger than the largest time in Fig. 1.6. 
1.8 Case of Fig. 1.6 (f) (steady-state). (o) LBM simulation of nondimensional density and pressure profiles across the domain mid-

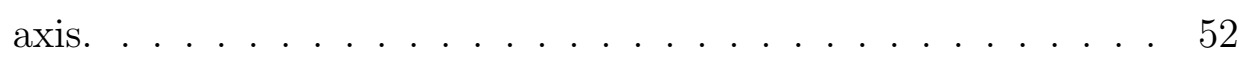

1.9 Terminal shape and flow velocity vectors for a bubble of density $\rho_{2}^{\prime}=6$ rising under buoyancy in a fluid with density $\rho_{1}^{\prime}=6000$, $\mu_{1}^{\prime} / \mu_{2}^{\prime}=1000$ : (rightward panels) VOSET results of [33]; (leftward panels) present LB results (see Table 1.4). . . . . . . . . 53

1.10 Time evolution of a rising bubble shape for $\rho_{1}^{\prime} / \rho_{2}^{\prime}=1000$, Bo $=$ 100, Mo $=1000$ (case of Fig. 1.9 (c)). From left to right, for $t^{\prime}=0,10^{3}, 2.10^{3}, 4.10^{3}$. Note, only half the domain height is plotted. . . . . . . . . . . . . . . 54

1.11 Rayleigh-Taylor instability problem for $\rho_{1} / \rho_{2}=3, A=0.5$, $\operatorname{Re}=256$. Time evolution of the two-fluid interface for four dimensionless times $t^{\prime}=t / \sqrt{L / g}$ : (leftward panels) results of [8]; (rightward panels) present LB results. . . . . . . . . . . 55

1.12 Definition sketch for initial interface profile of a large amplitude sinusoidal wave. . . . . . . . . . . . . . . . . 56

1.13 Space-periodic wave breaking (Fig. 1.12). Time evolution of an overturning breaking wave with $H / L=0.13$ and $h / L=0.25$ (dimensionless time $\left.t^{\prime}=t / \sqrt{L / g}\right) . \ldots \ldots \ldots \ldots$

2.1 Time evolution of the 3D simulation of a droplet impacting a thin layer of fluid, at four different times $t$, for $\rho_{1} / \rho_{2}=1000$, $\mu_{1} / \mu_{2}=100, R e=200$ and $W e=8000 . \ldots \ldots \ldots \ldots$ 
2.2 Spread factor $r / 2 R$ as a function of $U t / 2 R$ in the 3D simulation of a droplet impacting a thin layer of fluid, for $\rho_{1} / \rho_{2}=1000$, $\mu_{1} / \mu_{2}=100, R e=200$ and $W e=8,000$. The straight line corresponds to the power law $r=\sqrt{2 U t R} \ldots \ldots \ldots 78$

2.3 Domain and parameter definition for the quasi-2D simulation of a droplet impacting a thin fluid layer, following Lee et al. [8]. 79

2.4 Time evolution in quasi-2D simulations of a droplet impacting a thin fluid film for $\mathrm{Ut} / 2 \mathrm{R}=0.0,0.1,0.2,0.4,0.8,1.6$. $R e=20$, $W e=8000, \rho_{1} / \rho_{2}=1000$ and $\nu_{1} / \nu_{2}=40$; top: Lee et al.'s results [8], bottom: current LBM model. . . . . . . . . . . 79

2.5 Time evolution in quasi-2D simulations of a droplet impacting a thin fluid film for $\mathrm{Ut} / 2 \mathrm{R}=0.0,0.1,0.2,0.4,0.8,1.6$. $R e=100$, $W e=8000, \rho_{1} / \rho_{2}=1000$ and $\nu_{1} / \nu_{2}=40$; top: Lee et al.'s results [8], bottom: current LBM model. . . . . . . . . . . 80

2.6 Time evolution in quasi-2D simulations of a droplet impacting a thin fluid film for $\mathrm{Ut} / 2 \mathrm{R}=0.0,0.1,0.2,0.4,0.8,1.6$. $R e=500$, $W e=8000, \rho_{1} / \rho_{2}=1000$ and $\nu_{1} / \nu_{2}=40 ;$ top: Lee et al.'s results [8], bottom: current LBM model. . . . . . . . . . . . 8

2.7 Time evolution in quasi-2D simulations of a droplet impacting a thin fluid film, for $R e=2000, W e=8000, \rho_{1} / \rho_{2}=1000$ and $\nu_{1} / \nu_{2}=40$, using current LBM model. . . . . . . . . . 82

2.8 Time evolution of a rising bubble of fluid 2 into fluid 1 , for case (C), with $E t=243, M o=266, t^{*}=\sqrt{g / D} t, \rho_{1}^{\prime} / \rho_{2}^{\prime}=1000$, and $\mu_{1}^{\prime} / \mu_{2}^{\prime}=100 \ldots \ldots \ldots \ldots \ldots \ldots \ldots$ 
2.9 Rising bubble of fluid 2 into fluid 1: non-dimensional rising velocity $U^{*}=u / \sqrt{g D}$ as a function of non-dimensional time $t^{*}=\sqrt{g / D} t: 3 \mathrm{D}$ LBM results $(\cdot)$; experimental results of $[24]$ $(-) \ldots \ldots \ldots \ldots \ldots \ldots$. . . . . . . . . . . . .

2.10 Definition sketch in the vertical plane $(x, z)$ for the initial interface profile of a large amplitude sinusoidal wave (left), used to initialize the 3D-LBM domain (right). . . . . . . . . . . . 91

2.11 Time evolution of a 3D breaking sinusoidal wave with initial characteristics: $H / L=0.13$ and $h / L=0.13, R e=32,400$. Comparison of pre-breaking surface profiles in the main vertical cross-section $(y=0$; Figure 2.10), at $t / T=0.17,0.3,0.38$, and 0.46 (top to bottom) in : (left, right) 3D-LBM results in grids 1 and 2, respectively; (middle) Lubin et al.'s 3D-NS-VOF results

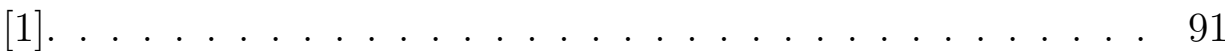

2.12 Convergence study for the case of Fig. 2.11. Geometry of the air-water interface in the main cross-section $(y=0)$ for four different resolutions in a grid with dimension ratios 8:4:1 and number of cells in the $x$ direction: (1; blue; grid 2) 512; (2; red) 400; (4; black) 256; and (6; green) 128. . . . . . . . . . . 94

2.13 Case of Fig. 2.12. $L_{2}$ relative error norm, between surface profiles computed for resolution (1) and those for resolutions (2-6), as a function of resolution in the $x$ direction . . . . . . . . 95 
2.14 Case of Fig. 2.11. Time evolution of a 3D overturning breaking wave in the middle lateral cross section $(y=0$; Figure 2.10). Comparison of details of splash-up generation in the main vertical cross-section $(y=0$; Figure 2.10) at $t / T=0.48,0.5,0.52$, $0.56,0.64,0.68$ (top to bottom) in : (left, right) 3D-LBM results in grids 1 and 2, respectively; (middle) Lubin et al.'s 3D-NSVOF results $[1] \ldots \ldots \ldots \ldots$

2.15 Time evolution of a 3D periodic breaking wave for the same case as in Figure 2.11, for grid 1 with $256 \times 128 \times 64$ cells in the $x, y$ and $z$ directions. . . . . . . . . . . . . . . 96

2.16 Time evolution of a 3D periodic breaking wave for the same case as in Figure 2.11, for grid 2 with $512 \times 64 \times 128$, in the $x, y$ and $z$ directions. . . . . . . . . . . . . . . . 97

2.17 Time evolution of a 3D periodic breaking wave for the same case as in Figure 2.11, for grid 3 with $400 \times 100 \times 100$, in the $x, y$ and $z$ directions. . . . . . . . . . . . . . . . . . . . . 98

3.1 D3Q19 sub-lattice model used in the present LBM, where the blue vectors indicate the 18 possible vectors of motion (both direction and length) for a particle to move on the lattice according to Eq. (3.2). . . . . . . . . . . . . . . 114

3.2 No-slip boundary condition at solid walls: sketch of LBM bounce back on link scheme. . . . . . . . . . . . . . . . . 118

3.3 Sketch of computational domain for 3D-DNS LBM modeling of turbulent channel flows. . . . . . . . . . . . . . . . . 118 
3.4 Sketch of half the computational domain in the wall-normal direction $z$, using the bounce back on link boundary condition. Total number of nodes for the half channel is $\delta / \Delta x=\delta \ldots . .121$

3.5 Validation of turbulent channel flow simulations. Mean streamswise velocity: KMM (solid-blue line), case A (dotted-black line), case B (dashed-blue line), case C (dashed-red line). . . . . . . 128

3.6 Validation of turbulent channel flow simulations. Reynolds shear stress $-\overline{u^{\prime} u^{\prime}}$, normalized by the wall shear velocity: KMM (solidblue line), case A (dotted-black line), case B (dashed-blue line), case C (dashed-red line). . . . . . . . . . . . . . . . . . 129

3.7 Validation of turbulent channel flow simulations. Root-meansquare velocity fluctuations normalized by the wall shear stress, : KMM (solid-blue line), case A (dotted-black line), case B (dashed-blue line), case C (dashed-red line). . . . . . . . . 130

3.8 Turbulent particle-laden flow simulations. Particle effects on

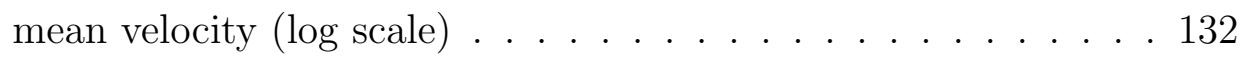

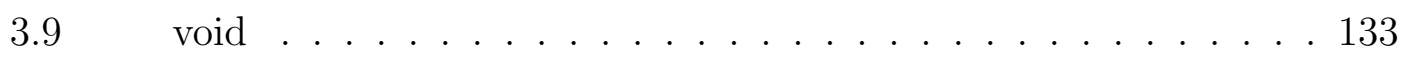

3.10 Velocity fluctuations . . . . . . . . . . . . . 135

3.11 Turbulent particle-laden flow simulations. Particle force and force balance . . . . . . . . . . . . . . . . . . 139

3.12 Production terms . . . . . . . . . . . . . . . . . . 142

3.13 Schematics of an array of quasi-streamwise vortices (QSV) (from

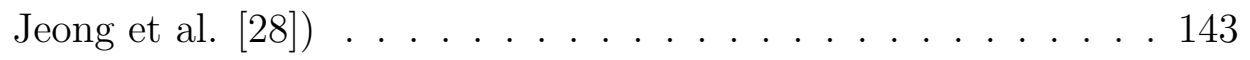

3.14 Instantaneous streamwise velocity and vorticity $\ldots \ldots \ldots . .144$ 
3.15 Turbulent particle-laden flow simulations. Snapshots of velocity fluctuations in horizontal $(x-y)$ planes. Clean channel flow (left plots), case IV (right plots) . . . . . . . . . . . . . 146

E.1 Poiseuille flow, blue lines current result, red line, analytical profile172

E.2 Velocity profiles at different times of unsteady Couette flow and the computational mesh ............... 173

E.3 Profiles of normalized velocity component $u$ through the geometric center of the cavity at $\mathrm{Re}=400$ circle-result of Lee et all 2001 blue line-current result . . . . . . . . . . . . . . . . 173 


\section{LIST OF TABLES}

1.1 Performance for different test cases presented in the application

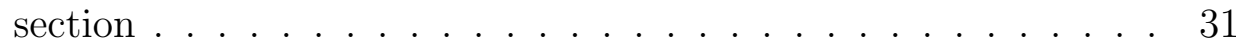

$1.2 \quad L_{2}$-norm error (between analytical and LB results) and body force magnitude $g^{\prime}$, as a function of LBM discretization size $h^{\prime}$, for the two-fluid Poiseuille flow test case of Fig. 1.3 (see, Fig. 1.4 for error plot). . . . . . . . . . . . . . . . . 37

1.3 Stationary bubble case. Comparison of computed and analytical $\left(0.4 \mathrm{~N} / \mathrm{m}^{2}\right)$ pressure jumps $\Delta p$ for stationary circular droplets, as a function of LBM discretization. . . . . . . . . . . . . 40

1.4 LBM parameters for rising bubble computations. . . . . . . . . 43

2.1 Rise of a 3D bubble of a light fluid 2 in a heavy fluid $1\left(\rho_{1}^{\prime} / \rho_{2}^{\prime}=\right.$ $\left.1000 ; \mu_{1}^{\prime} / \mu_{2}^{\prime}=100\right)$. Comparison of terminal Reynolds number between experiments [22] and 3D-LBM numerical simulations.

2.2 Comparison of the computed terminal shapes of a rising bubble of fluid 2 into fluid 1, with the experimental results of [22] and the numerical simulations of Hua et al.[24], for $\rho_{1}^{\prime} / \rho_{2}^{\prime}=1000$ and $\mu_{1}^{\prime} / \mu_{2}^{\prime}=100 \ldots \ldots \ldots \ldots \ldots$. . . . . . . . . . 85

$3.1 \quad$ Particle properties . . . . . . . . . . . . . 127

3.2 DNS runs legend . . . . . . . . . . . . . . . 128 


\title{
CHAPTER 1
}

\section{Efficient GPGPU implementation of a lattice Boltzmann model for multiphase flows with high density ratios}

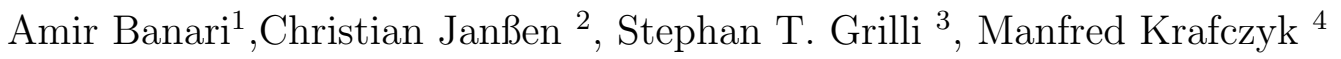 \\ Published in Computer and Fluids 93 (2014) 1-17.
}

\footnotetext{
${ }^{1}$ Ocean Engineering Department, University of Rhode Island, E-mail: amir_banari@my.uri.edu

${ }^{2}$ Institute for Fluid Dynamics and Ship Theory, Hamburg University of Technology, Germany, E-mail: mail@christian-janssen.de

${ }^{3}$ Ocean Engineering department, University of Rhode Island, E-mail: grilli@oce.uri.edu

${ }^{4}$ Institute for Computational Modeling in Civil Engineering, Technische Universitaet Braunschweig, Germany, E-mail: kraft@irmb.tu-bs.de
} 


\section{Abstract}

We present the development of a Lattice Boltzmann Method (LBM) for the numerical simulation of multiphase flows with high density ratios, such as found in ocean surface wave and air-sea interaction problems, and its efficient implementation on a massively parallel General Purpose Graphical Processing Unit (GPGPU). The LBM extends Inamuro's et al.'s [T. Inamuro, T. Ogata, S. Tajima and N. Konishi, A lattice Boltzmann method for incompressible two-phase flows with large density differences, J. Comp. Phys., 198 (2004) 628-644.] multiphase method by solving the Cahn-Hilliard equation on the basis of a rigorously derived diffusive interface model. Similar to Inamuro et al., instabilities resulting from high density ratios are eliminated by solving an additional Poisson equation for the fluid pressure. We first show that LBM results obtained on a GPGPU agree well with standard analytic benchmark problems for: (i) a two-fluid laminar Poiseuille flow between infinite plates, where numerical errors exhibit the expected convergence as a function of the spatial discretization; and (ii) a stationary droplet case, which validates the accuracy of the surface tension force treatment as well as its convergence with increasing grid resolution. Then, simulations of a rising bubble simultaneously validate the modeling of viscosity (including drag forces) and surface tension effects at the fluid interface, for an unsteady flow case. Finally, the numerical validation of more complex flows, such as Rayleigh-Taylor instability and wave breaking, is investigated. In all cases, numerical results agree well with reference data, indicating that the newly developed model can be used as an accurate tool for investigating the complex physics of multiphase flows with high density ratios. Importantly, the GPGPU implementation proves highly efficient for this type of models, yielding large speed-ups of computational time. Although

only two-dimensional cases are presented here, for which computational effort is 
low, the LBM model can (and will) be implemented in three-dimensions in future work, which makes it very important using an efficient solution. Lattice Boltzmann method, multiphase flows, high density ratio, GPGPU parallel implementation

\section{$1.1 \quad$ Introduction}

The numerical simulation of multiphase and multi-component fluid flows is a challenging problem in Computational Fluid Dynamics (CFD), both for conventional macroscopic and mesoscopic methods, such as the Lattice-Boltzmann Method (LBM). In classical CFD methods, multiphase flows are simulated by coupling a Navier-Stokes (NS) equation solver to an advection or advection-diffusion equation scheme, for the updating of interfaces between fluids [1]. In earlier work, advection equations have been used in combination with either sharp or diffuse interface models (although this may seem less adequate in the latter case), whereas advection-diffusion equations have mostly been used with diffusive interface models. The interface itself is typically represented by a capturing method such as the widely used Volume Of Fluid (VOF) method [2], or an interface tracking method. Most of the interface tracking methods assume a sharp interface, i.e., they consider the phase transition to be clearly defined and thus the interface between two fluids to be infinitely thin. By contrast, the interface capturing methods allow for both sharp or diffusive interface representations, depending on the equation solved. An additional challenge when using a sharp interface method is the accurate computation of the interface curvature and related surface tension forces. This has encouraged many researchers to use diffusive interface methods, in which surface tension forces at interfaces are modeled as a continuum, by distributing them over thin but numerically resolved layers [3]. Such models have recently attracted much interest, owing to their computational advantages [4, 5]. Because of these various options, when developing and implementing a free surface or multi- 
phase CFD model, one has to make a priori decisions regarding using: (i) a sharp or diffusive interface method; (ii) an advection or advection-diffusion equation for free surface updating; and (iii) a tracking or a capturing method for the interface representation. In our proposed two-phase model, detailed below, interface motion is modeled by a Cahn-Hilliard's (CH) interface capturing, advection-diffusion equation [6], using a scalar order parameter $\phi_{\alpha}(\alpha=1,2)$ to identify each phase. The interface between the two phases is then defined as a smooth transition from $\phi_{1}$ to $\phi_{2}$ and vice versa.

Recently, the LBM has matured into a powerful alternative to classical NS solvers, both for simulating single phase, and multiphase and multi-component flows [7, 8, 9, 10]. The LBM discretizes the Boltzmann equation, which governs the dynamics of molecular probability distribution functions from a microscopic scale point of view, based on a discrete velocity set. This yields a numerical method for computing macroscopic distribution functions on a Cartesian grid (the lattice). Macroscopic hydrodynamic quantities, such as pressure and velocity, are obtained as low-order moments of these distribution functions. The resulting formulation can be shown to converge towards the solution of classical macroscopic equations such as NS, with second order in space and first order in time [11]. The LBM has several solver-specific features, which allow taking full advantage of recent advances in massively parallel General Purpose Graphical Processing Units (GPGPU) [12], such as a an operator locality and fairly regular algorithms, which yield significantly more efficient parallel codes than those of more traditional CFD solvers.

While there have been numerous applications of classical CFD solvers to multiphase flows, whose exhaustive review is beyond the scope of this paper, over the past two decades, several noteworthy methods have been developed for simulating multiphase flows in the context of the LBM. These are: Rothman and Keller's 
color method [13], the Shan and Chen model (SC) [14], Swift's free energy method [7], and the method of He et al. [8]. In the $\mathrm{SC}$ method, separate probability distribution functions are introduced for each phase, and these are modified by a forcing term that models "molecular" interactions with neighboring lattice nodes in the other phase. Swift et al. used a free energy concept, in which the stress tensor is modified by adding the effects of surface tension forces. In their method, two sets of particle distribution functions are required, one for solving NS equations and one for solving the approximate $\mathrm{CH}$ interface capturing equation. He et al. transformed the classical discrete Boltzmann equation for a single phase, from a mass and momentum to a pressure and momentum formulation. This transformation helps reducing potential instabilities due to high gradients in fluid density near the interface. Similar to Swift et al., a second set of particle distribution functions is used to track the interface.

While all of the above methods successfully solved multiphase flows, the maximum fluid density ratio achievable in computations was limited by the occurrence of instabilities for high density ratios (typically larger than 10-20). Developing methods to overcome this limitation is challenging and represents an active research area in LBM. In this work, we aimed at developing an accurate and efficient LBM method for investigating the complex physics of ocean wave and air-sea interaction processes. Hence, our method must deal with large density ratios of about 1,000. Our proposed approach builds and improves on some recent progress achieved in the LBM modeling of multiphase flows. In particular:

- Lee et al. [9] used an approach similar to that of He et al. [8] to solve discrete Boltzmann equations for the pressure and momentum in multiphase flows. In those, they split up intermolecular forces for non-ideal gas into hydrodynamic pressure, thermodynamic pressure, and surface tension force 
contributions. They reported that "parasitic currents" affected numerical results at interfaces, due to the imbalance between thermodynamic pressure and surface tension forces resulting from truncation errors, particularly, in relation to curvature computations. They nearly eliminated this problem by using a thermodynamic identity to recast the intermolecular forcing term from a stress to a potential form. Furthermore, they used different discretization patterns (i.e., centered, staggered, and mixed differences) at different steps of the simulation, to make their numerical scheme stable for large density ratios. With this discretization scheme, they were able to simulate two phase flows with density ratio of up to 1,000. However, their method was only valid for low Reynolds and Mach numbers. Additionally, numerical efficiency seemed to be quite low, due to the need for calculating various forms of first- and second-order derivatives of the macroscopic variables.

- Inamuro et al.'s [10] LBM method overcame numerical instabilities resulting from high density ratios by removing density from the advection part of the equilibrium distribution functions, resulting in the absence of a pressure gradient in the momentum equation (referred to in the following as "pressureless" NS equations). They then corrected the velocity field by solving a Poisson equation for the pressure. Unlike in classical LBMs, in their model, the fluid viscosity is not related to the relaxation time, because of the absence of pressure and density in the equilibrium distribution functions. Therefore, viscous effects are modeled by adding: (i) an extra term to the equilibrium distribution functions, which removes the dependency of relaxation time on viscosity; (ii) the viscous stress tensor as a body force to the collision operator. However, specifying viscous effects this way in the model yields additional nonphysical terms in the corresponding momentum equation, which 
decrease the model accuracy.

In this work, we developed and implemented a LBM based in part on Inamuro et al.'s [10, 15] approach of removing the pressure gradient from the momentum equation. However, in our model we use a modified primary set of equilibrium functions for the "pressureless" NS equations, in which viscosity is still present and related to the relaxation time as in a classical LBM. Thus in our method, the corresponding NS equations do not have the undesired terms that appear in Inamuro et al.'s model. Additionally, Inamuro et al. used a convection-diffusion equation in their interface capturing method, whose theoretical derivation did not seem to be fully rigorous. By contrast, to this effect, we use the standard CahnHilliard $(\mathrm{CH})$ equation, in which surface tension and equivalent body forces are rigorously derived, and we solve it using an LBM scheme, by way of a second set of equilibrium functions; this yields a more accurate and efficient solution than in earlier implementations, particularly on a GPGPU. Finally, we similarly correct the "pressureless" velocity field by solving a Poisson equation for the pressure, but here this is done by way of a third set of LBM equilibrium functions, again providing an efficient scheme when implemented on a GPGPU.

More specifically, it has been demonstrated in various publications [16, 12, 17, 2, 18] that LBM methods can be made very efficient when implemented on massively parallel GPGPUs (single and multiple units). Hence, as indicated above, our proposed model's algorithm, which uses 3 separate sets of LB equilibrium functions and related collision-propagation operators is optimally formulated for such an implementation. Accordingly, we developed our LBM code in the nVIDIA CUDA framework, which made it possible efficiently implementing and validating it on the latest generation GPGPUs (e.g., nVIDIA Tesla C2070, which provide up to 448 cores, $6 \mathrm{~GB}$ of main memory, and a double precision computing capability). 
For all applications presented herein, this GPGPU implementation led to computational speedups of about two orders of magnitude, as compared to a single-core CPU implementation of the same model. However, because the Poisson equation must be (iteratively) solved over the entire computational domain for each time step of the solution, these are still very demanding computations and only twodimensional (2D) problems have been solved so far, on a single GPGPU, although the method could be quite easily extended to three dimensions and to multiple GPGPUs.

The paper is organized as follows. Section 1.2 provides an introduction to the free energy method, applied to diffusive interfaces modeled using the $\mathrm{CH}$ equation. Our proposed LBM for multiphase flows with high density ratio is detailed in Section 1.3, where we separately describe the LB solution of the momentum, CH advection-diffusion, and pressure Poisson equations. The GPGPU implementation is briefly described in Section 1.4. In Section 1.5, the method is validated by comparing 2D numerical results to reference solutions for two-component Poiseuille flows, stationary droplets of one fluid embedded in another, a bubble of a lighter fluid rising in another fluid, the Rayleigh Taylor instability, and breaking ocean surface waves. Finally, Section 1.6 offers conclusions and perspectives for future work.

\subsection{Diffusive interface models}

As mentioned in the introduction, numerical schemes based on a sharp interface representation, while usually more accurate, may require addressing additional numerical problems in their implementation, as compared to diffusive interface models. In particular, although not strictly necessary, sharp interface models may use a moving numerical grid, whereas diffusive interface models naturally accommodate fixed grids (such as used in the LBM). Sharp interface models also face 
difficulties for accurately computing the interface curvature and the related surface tension forces. This often leads to the appearance of "parasitic currents" in the numerical solution along the interface. These problems disappear when using a diffusive-interface representation based on the continuous variation of an order parameter (such as density or a function of density), in a way that is physically consistent with microscopic theories of interfacial processes. Three main types of diffusive-interface models have been proposed in the literature: (i) tracking force models [4]; (ii) continuum surface force models [5]; and (iii) phase-field models [3].

In the current work, we use the latter approach, in which the total free energy $\mathcal{F}$ of a two-fluid system is specified to be minimum for the equilibrium interface profile $\phi(\zeta)$, where $\phi$ denotes a continuously varying order parameter (with values $\phi_{1}$ and $\phi_{2}$ referring to fluid 1 and 2 on either side of the interface, respectively; and $\left.\phi_{1}>\phi_{2}, \phi \in\left[\phi_{1}, \phi_{2}\right]\right)$, and $\zeta$ is a coordinate normal to the interface (positive when pointing from fluid 1 to 2). More specifically, Cahn [6] expressed the free energy density of an isothermal two-phase/fluid system as,

$$
f=\frac{k}{2}|\nabla \phi|^{2}+\beta \Psi(\phi)
$$

The first term in this equation is related to the energy gradient and the second one to the bulk free-energy density $\Psi(\phi)$. In the following, we will express the two parameters $\beta$ and $k$ in Eq. (1.1) as a function of the standard surface tension coefficient $\sigma_{12}$ of the two fluid system and an assumed interface thickness $W$.

The existence of two phases is possible if $\Psi$ has two minima, such as when posing, $\Psi(\phi)=\left(\phi-\phi_{2}\right)^{2}\left(\phi-\phi_{1}\right)^{2}$. Based on Eq. 1.1), the total free-energy of the two-phase system in domain $\Omega$ reads,

$$
\mathcal{F}=\int_{\Omega} f(\phi, \nabla \phi) d \Omega=\int_{\Omega}\left\{\frac{k}{2}|\nabla \phi|^{2}+\beta \Psi(\phi)\right\} d \Omega .
$$

The chemical potential $\mu_{\phi}$ is then defined as the functional derivative of the free energy with respect to $\phi$, which can be easily derived from the Euler-Lagrange 
equation as,

$$
\mu_{\phi}=\frac{\delta \mathcal{F}}{\delta \phi}=\frac{\partial f}{\partial \phi}-\nabla\left(\frac{\partial f}{\partial(\nabla \phi)}\right)=\beta \Psi^{\prime}-k \nabla^{2} \phi
$$

As indicated, the equilibrium state of the interface is defined such that the variation of $\phi$ across the interface minimizes $\mathcal{F}$, hence, this corresponds to $\mu_{\phi}=0$ or,

$$
k \frac{d^{2} \phi}{d^{2} \zeta}=\beta \frac{d \Psi}{d \phi}
$$

since $\phi$ is only a function of $\zeta$. Multiplying both sides of this equation by $d \phi / d \zeta$ and integrating with respect to $\zeta$ yields,

$$
k\left(\frac{d \phi}{d \zeta}\right)^{2}=2 \beta \Psi
$$

which, combined with the above definition of $\Psi(\phi)$, can be solved for $\phi$ as,

$$
\phi(\zeta)=\frac{\phi_{1}+\phi_{2}}{2}+\frac{\phi_{1}-\phi_{2}}{2} \tanh \left(\frac{2 \zeta}{W}\right),
$$

where the equivalent interface thickness has been defined as,

$$
W=\frac{4}{\phi_{1}-\phi_{2}} \sqrt{\frac{k}{2 \beta}} .
$$

This equation predicts that, at a distance $W / 2$ on either side of the interface, the order parameter reaches $76 \%$ of its value in each fluid, $\phi_{1}$ or $\phi_{2}$, respectively.

Jacqmin [3] further expressed surface tension forces as a function of the variation of the order parameter across the interface (such as in Eq. (1.6), by assuming that the rate of change of $\mathcal{F}$ due to convection is equal and opposite to the rate of change of the kinetic energy due to surface tension forces $\boldsymbol{F}$, i.e.,

$$
\int_{\Omega} \boldsymbol{F} \cdot \boldsymbol{u} d \Omega=\int_{\Omega} \mu_{\phi} \nabla \cdot(\phi \boldsymbol{u}) d \Omega=-\int_{\Omega}\left(\phi \nabla \mu_{\phi}\right) \cdot \boldsymbol{u} d \Omega
$$

where $\boldsymbol{u}$ denotes the interface velocity. Using Eq. (1.3) and noting that surface tension forces only exist within the plane tangent to the interface, yields,

$$
\boldsymbol{F}=-\phi \nabla \mu_{\phi}=k \phi \nabla\left(\nabla^{2} \phi\right) .
$$


Eq. (1.9) represents surface tension by an equivalent volume force, which could be directly inserted into the governing momentum (NS) equations (see, e.g., [3]).

Introducing a stress representation of surface tension forces into Eq. (1.9), Jacqmin showed that, for a plane interface, the equivalent surface tension coefficient for the two-fluid system can be calculated as,

$$
\sigma_{12}=k \int_{-\infty}^{+\infty}\left(\frac{d \phi}{d \zeta}\right)^{2} \mathrm{~d} \zeta
$$

Combining Eqs. (1.10) and (1.6), we find,

$$
\sigma_{12}=\frac{\left(\phi_{1}-\phi_{2}\right)^{3}}{6} \sqrt{2 k \beta}
$$

Hence, in a given application, once the interface thickness $W$ and the surface tension coefficient $\sigma_{12}$ are specified, the two governing parameters of the diffusive interface model, $\beta$ and $k$, can be calculated with Eqs. (1.7) and (1.11).

Finally, the motion of the diffusive interface is modeled, following Jacqmin [3], as a function of the order parameter by extending the Cahn-Hilliard $(\mathrm{CH})$ equation [6] to include convection, as,

$$
\frac{\partial \phi}{\partial t}+\nabla \cdot(\phi \boldsymbol{u})=M \nabla^{2} \mu_{\phi}
$$

where the right hand side represents the interface diffusive transport, expressed as a function of a mobility coefficient $M$ and the chemical potential $\mu_{\phi}$, defined above.

\subsection{Lattice Boltzmann Model}

In this work, two-dimensional (2D) multiphase flows are simulated by solving two sets of equations: (i) the NS equations, which provide the flow fields, based on the conservation of mass and momentum, with the addition of the volumetric surface tension force term of Eq. (1.9); (ii) the extended Cahn-Hilliard Eq. (1.12), which describes the interface motion. We solve these equations using a new Lattice 
Boltzmann method (LBM), which is an extension of Inamuro et al.'s [10] method, developed to accurately simulate multiphase flows with large density ratios (such as air-water). As will be detailed in the following, our algorithms significantly differ from Inamuro et al.'s in several aspects.

To develop our LBM equations, we first introduce two sets of LB particle distribution functions, one for each equation (i) and (ii), and then find the corresponding mesoscopic equilibrium distribution functions, which reproduce the desired macroscopic equations. To discretize the 2D-LBM equations, we use the so-called D2Q9 set of particle velocities (Fig. 1.1), which is based on 9 discrete particle velocities in directions $\mathbf{e}_{i}$ defined as [19],

$$
\begin{array}{ll}
\boldsymbol{e}_{0}=(0,0) & \\
\boldsymbol{e}_{i}=c(\cos ((i-1) \pi / 4), \sin ((i-1) \pi / 4)) ; & \mathrm{i}=1,3,5,7 \\
\boldsymbol{e}_{i}=\sqrt{2} c(\cos ((i-1) \pi / 4), \sin ((i-1) \pi / 4)) ; & \mathrm{i}=2,4,6,8
\end{array}
$$

with $\Delta x$ and $\Delta t$ the lattice constant mesh and time step sizes, respectively, and $c=\Delta x / \Delta t$ defining the particle propagation speed on the lattice.

In the LBM, it is customary to use non-dimensional lattice variables (here denoted by a prime) scaled on the basis of a length scale $\lambda$, time scale $\tau$ and mass scale $\varpi$; thus, for the mesh parameters, $\Delta x^{\prime}=\Delta x / \lambda, \Delta t^{\prime}=\Delta t / \tau$ and $c^{\prime}=c \tau / \lambda$. It is also customary to assume that $c^{\prime}=1$, which is akin to having the mesh Courant number be unity. If the length scale is further defined as $\lambda=\Delta x$, we then have $\Delta x^{\prime}=1$, which requires $\tau=\Delta t$ and $\Delta t^{\prime}=1$ as well. Hence, with these definitions, in lattice variables, we always have $c^{\prime}=\Delta x^{\prime}=\Delta t^{\prime}=1[19]$. 


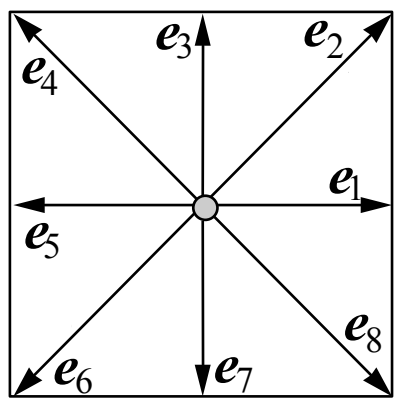

Figure 1.1: D2Q9 Lattice for definition of particle velocities

\subsubsection{Lattice Bolzmann solution of momentum equation Classical LBM solution of NS equations}

The macroscopic continuity and momentum (i.e., NS) equations for compressible isothermal fluids read (using tensor notations),

$$
\begin{gathered}
\frac{\partial \rho}{\partial t}+\rho \frac{\partial u_{\alpha}}{\partial x_{\alpha}}=0 \\
\rho\left\{\frac{\partial u_{\alpha}}{\partial t}+u_{\beta} \frac{\partial u_{\alpha}}{\partial x_{\beta}}\right\}=\frac{\partial \sigma_{\alpha \beta}}{\partial x_{\beta}}+B_{\alpha}
\end{gathered}
$$

where $\rho(\phi)$ is the local density of the two-fluid system, $B_{\alpha}$ is a body force (e.g., gravity: $B_{\alpha}=\rho g_{\alpha}$ ), and $\sigma_{\alpha \beta}$ denotes the stress tensor, which, for two-phase flow problems, can be decomposed into three parts [9],

$$
\sigma_{\alpha \beta}=-p \delta_{\alpha \beta}+\sigma_{\alpha \beta}^{v i s c}+\sigma_{\alpha \beta}^{S T}
$$

where $p$ is pressure, $\sigma_{\alpha \beta}^{\text {visc }}$ the viscous stress tensor, and $\sigma_{\alpha \beta}^{S T}$ a stress tensor representing the volumetric effects of surface tension forces at the two fluid interface. For Newtonian fluids, these tensors read,

$$
\begin{aligned}
\sigma_{\alpha \beta}^{v i s c} & =\mu\left(\frac{\partial u_{\alpha}}{\partial x_{\beta}}+\frac{\partial u_{\beta}}{\partial x_{\alpha}}\right) \\
\sigma_{\alpha \beta}^{S T} & =\left(\frac{k}{2} \frac{\partial \phi}{\partial x_{\gamma}} \frac{\partial \phi}{\partial x_{\gamma}}+k \phi \frac{\partial^{2} \phi}{\partial x_{\gamma} \partial x_{\gamma}}\right) \delta_{\alpha \beta}-k \frac{\partial \phi}{\partial x_{\alpha}} \frac{\partial \phi}{\partial x_{\beta}},
\end{aligned}
$$

where $\mu(\phi)$ denotes the local dynamic viscosity, and the second equation directly follows from the definition of surface tension forces in Eq. (1.9) (i.e., $\left.F_{\alpha}=\partial \sigma_{\alpha \beta}^{S T} / \partial x_{\beta}\right)$ 
Lee et al. 9], however, reported that numerical schemes where the stress tensor is directly based on Eqs. (1.16) to (1.18) are often unstable, as a result of an imbalance of the pressure and surface tension terms due to truncation errors, yielding parasitic currents near the phase interface. To alleviate this problem, they introduced a modified pressure $p^{m}$, which includes parts of the surface tension effects,

$$
p^{m}=p+\frac{k}{2} \frac{\partial \phi}{\partial x_{\gamma}} \frac{\partial \phi}{\partial x_{\gamma}}-k \phi \frac{\partial^{2} \phi}{\partial x_{\gamma} \partial x_{\gamma}},
$$

and a tensor $\sigma_{\alpha \beta}^{S T m}$ also modified accordingly. They showed in applications that the variation of the modified pressure is now smooth across the phase interface, compared to that of pressure $p$, which greatly improves the stability of the numerical model in the case of large surface tension forces.

With these new definitions the total stress tensor is reformulated as,

$$
\sigma_{\alpha \beta}=-p^{m} \delta_{\alpha \beta}+k\left(\frac{\partial \phi}{\partial x_{\gamma}} \frac{\partial \phi}{\partial x_{\gamma}} \delta_{\alpha \beta}-\frac{\partial \phi}{\partial x_{\alpha}} \frac{\partial \phi}{\partial x_{\beta}}\right)+\mu\left(\frac{\partial u_{\alpha}}{\partial x_{\beta}}+\frac{\partial u_{\beta}}{\partial x_{\alpha}}\right) .
$$

We now introduce a set of particle distribution functions $g_{i}(\boldsymbol{x}, t)$ to satisfy the equations of conservation of mass (1.14) and momentum (1.15), with the stress tensor defined by Eq. (1.20). In a standard LBM ansatz, the time evolution of these particle distribution functions is computed as (assuming a single relaxation time (SRT) formulation [20] $)(i=0, \ldots, 8)$,

$$
g_{i}\left(\boldsymbol{x}+\boldsymbol{e}_{i} \Delta t, t+\Delta t\right)=g_{i}(\boldsymbol{x}, t)-\frac{\Delta t}{\tau_{g}}\left(g_{i}(\boldsymbol{x}, t)-g_{i}^{(e q)}(\boldsymbol{x}, t)\right)+\Delta t B_{i},
$$

where $g_{i}^{(e q)}$ denotes the equilibrium state, to which the particle distribution functions are locally driven, and $B_{i}$ represents the effects of body forces $B_{\alpha}$ in Eqs. 1.15). Following Buick and Greated 21] the latter can be expressed as $B_{i}=w_{i} e_{i \alpha} B_{\alpha} / c_{s}^{2}$, where $w_{i}$ is a weight factor (defined later). The relaxation time $\tau_{g}$ is related to the fluid viscosity and the assumed speed of sound in the medium, $c_{s}$ (also detailed later). 
With a proper definition of the equilibrium distribution functions, such LBM schemes converge to the solution of NS equations [11]. In standard LBMs used for two-phase flows with low density ratios, the macroscopic values of fluid density, momentum, and stresses are obtained from the moments of the particle distribution functions [20]. Assuming no other body forces besides gravity, we have, respectively,

$$
\begin{aligned}
& \sum_{i=0}^{b} g_{i}^{e q}=\rho \\
& \sum_{i=0}^{b} g_{i}^{e q} e_{i \alpha}=\rho u_{\alpha} \\
& \sum_{i=0}^{b} g_{i}^{e q} e_{i \alpha} e_{i \beta}=\left(\rho c_{s}^{2}-k \frac{\partial \phi}{\partial x_{\gamma}} \frac{\partial \phi}{\partial x_{\gamma}}\right) \delta_{\alpha \beta}+k \frac{\partial \phi}{\partial x_{\alpha}} \frac{\partial \phi}{\partial x_{\beta}}+\rho u_{\alpha} u_{\beta}
\end{aligned}
$$

\section{Modified LBM for two fluids with high density ratio}

When using the classical LBM equations to simulate two fluid flows with high density ratio, the large density gradient near the interface between fluids will usually cause large truncation errors that could trigger numerical instabilities. To eliminate this problem, following Inamuro et al., we eliminate the bulk density from the previous equations, leading to modified NS equations, which no longer have a pressure gradient term (so-called pressureless NS equations). The velocity field $\boldsymbol{u}^{*}$ found as solution of these equations, however, will have to be corrected by solving an additional Poisson equation. This is detailed in the following.

First, we define a new equilibrium state where the moments of $g_{i}$ 's or $g_{i}^{(e q)}$ 's 
are defined without the fluid density $\rho$ as,

$$
\begin{aligned}
& \sum_{i=0}^{b} g_{i}^{(e q)}=1, \\
& \sum_{i=0}^{b} g_{i}^{(e q)} e_{i \alpha}=u_{\alpha}^{*}, \\
& \sum_{i=0}^{b} g_{i}^{(e q)} e_{i \alpha} e_{i \beta}=\left(c_{s}^{2}-\frac{k}{\rho} \frac{\partial \phi}{\partial x_{\gamma}} \frac{\partial \phi}{\partial x_{\gamma}}\right) \delta_{\alpha \beta}+\frac{k}{\rho} \frac{\partial \phi}{\partial x_{\alpha}} \frac{\partial \phi}{\partial x_{\beta}}+u_{\alpha}^{*} u_{\beta}^{*},
\end{aligned}
$$

and the new equilibrium distribution functions for a D2Q9 model, which both modify and extend the classical formulation (e.g., [22]), read $(i=0, \ldots, 8)$,

$$
g_{i}^{(e q)}=w_{i}\left\{1+\frac{e_{i \alpha} u_{\alpha}^{*}}{c_{s}^{2}}+\frac{\left(e_{i \alpha} u_{\alpha}^{*}\right)^{2}}{2 c_{s}^{4}}-\frac{\left|\boldsymbol{u}^{*}\right|^{2}}{2 c_{s}^{2}}\right\}+w_{i} \frac{k}{\rho} G_{\alpha \beta} e_{i \alpha} e_{i \beta}-v_{i} \frac{k}{2 \rho}|\nabla \phi|^{2} .
$$

where the summation is performed over indices $\alpha$ and $\beta$ (but not on $i$ ), $c_{s}$ is the speed of sound defined, for a D2Q9 lattice as, $c_{s}=c / \sqrt{3}$ [19], and $w_{i}$ and $v_{i}$ are weighting functions defined as,

$$
\begin{aligned}
& w_{0}=\frac{4}{9} ; w_{1,3,5,7}=\frac{1}{9} ; w_{2,4,6,8}=\frac{1}{36}, \\
& v_{0}=-\frac{5}{3 c^{2}} ; v_{i}=\frac{3}{c^{2}} w_{i}(i=1,2, \ldots, 8),
\end{aligned}
$$

and

$$
G_{\alpha \beta}(\phi)=\frac{9}{2 c^{4}} \frac{\partial \phi}{\partial x_{\alpha}} \frac{\partial \phi}{\partial x_{\beta}}-\frac{9}{4 c^{4}} \frac{\partial \phi}{\partial x_{\gamma}} \frac{\partial \phi}{\partial x_{\gamma}} \delta_{\alpha \beta}
$$

[Note, these values of $v_{i}$ and $G_{\alpha \beta}$ are similar to those of Inamuro et al.'s [10] and Swift et al.'s [23] model.]

It should be pointed out that, since the zeroth-order moments of equilibrium functions determine the pressure gradient in the resulting NS equations, setting these moments to an arbitrary constant (rather than to one as in the right-hand side of Eq. (1.25) ) would still lead to pressure-less momentum equations. The constant 
one in Eq. 1.25) is used here both for simplicity and for being consistent with classical LBMs, in which the first zeroth-order moment of $g_{i}^{(e q)}$ is set to the density, which usually fluctuates around a value of one (in LBM variables).

The convergence of the solution of these modified LBM equations to that of NS equations (without a pressure gradient term) is verified by applying the ChapmanEnskog expansion [11] to Eq. (1.21), with the equilibrium distribution functions of Eqs. 1.28 to 1.31. This leads to:

$$
\begin{aligned}
\frac{\partial u_{\alpha}^{*}}{\partial t}+u_{\beta}^{*} \frac{\partial u_{\alpha}^{*}}{\partial x_{\beta}} & =\frac{k}{\rho} \frac{\partial}{\partial x_{\beta}}\left\{\frac{\partial \phi}{\partial x_{\gamma}} \frac{\partial \phi}{\partial x_{\gamma}} \delta_{\alpha \beta}-\frac{\partial \phi}{\partial x_{\alpha}} \frac{\partial \phi}{\partial x_{\beta}}\right\} \\
& +\frac{\partial}{\partial x_{\beta}}\left\{c_{s}^{2}\left(\tau_{g}-\frac{1}{2} \Delta t\right)\left(\frac{\partial u_{\alpha}^{*}}{\partial x_{\beta}}+\frac{\partial u_{\beta}^{*}}{\partial x_{\alpha}}\right)\right\}+\frac{B_{\alpha}}{\rho},
\end{aligned}
$$

whose right-hand-side should be the gradient of the stress tensor defined in Eq. (1.20) without a pressure gradient term. As there is no density in the second term in the right-hand-side, however, the relaxation time $\tau_{g}$ cannot immediately be related to the fluid kinematic viscosity $\nu$, by contrast with a classical LBM [20]. To do so, we first have to rewrite the governing pressureless NS Eqs. 11.15 and 1.20 as,

$$
\begin{aligned}
\frac{\partial u_{\alpha}^{*}}{\partial t}+u_{\beta}^{*} \frac{\partial u_{\alpha}^{*}}{\partial x_{\beta}}= & \frac{k}{\rho} \frac{\partial}{\partial x_{\beta}}\left\{\frac{\partial \phi}{\partial x_{\gamma}} \frac{\partial \phi}{\partial x_{\gamma}} \delta_{\alpha \beta}-\frac{\partial \phi}{\partial x_{\alpha}} \frac{\partial \phi}{\partial x_{\beta}}\right\} \\
& +\frac{\partial}{\partial x_{\beta}}\left\{\frac{\mu}{\rho}\left(\frac{\partial u_{\alpha}^{*}}{\partial x_{\beta}}+\frac{\partial u_{\beta}^{*}}{\partial x_{\alpha}}\right)\right\} \\
& -\mu\left(\frac{\partial u_{\alpha}^{*}}{\partial x_{\beta}}+\frac{\partial u_{\beta}^{*}}{\partial x_{\alpha}}\right) \frac{\partial}{\partial x_{\beta}}\left(\frac{1}{\rho}\right)+\frac{B_{\alpha}}{\rho} .
\end{aligned}
$$

Now, Eq. (1.33) can be made identical to Eq. 11.32 if one defines $\mu / \rho=\nu=$ $c_{s}^{2}\left(\tau_{g}-\frac{1}{2} \Delta t\right)$, which is the standard relationship in classical LBMs (e.g., [20]), and adds the next to last term of its right-hand-side to the LBM evolution Eq. (1.21) as an equivalent body force. According to Buick and Greated's formulation, this 
reads,

$$
\begin{aligned}
g_{i}\left(\boldsymbol{x}+\boldsymbol{e}_{i} \Delta t, t+\Delta t\right) & =g_{i}(\boldsymbol{x}, t)-\frac{\Delta t}{\tau_{g}}\left(g_{i}(\boldsymbol{x}, t)-g_{i}^{(e q)}(\boldsymbol{x}, t)\right) \\
& +\frac{3}{c^{2}} w_{i} e_{i \alpha} \Delta t\left\{\frac{B_{\alpha}}{\rho}-\sigma_{\alpha \beta}^{v i s c, *} \frac{\partial}{\partial x_{\beta}}\left(\frac{1}{\rho}\right)\right\},
\end{aligned}
$$

where $\sigma_{\alpha \beta}^{v i s c, *}$ is given by Eq. (1.17), when using the pressureless velocity $\boldsymbol{u}^{*}$. It is noted that the last term in Eq. (1.34) is Buick and Greated's classical body force divided by density.

Based on the above Chapman-Enskog expansion, the relaxation time is thus expressed as,

$$
\tau_{g}=\frac{\nu}{c_{s}^{2}}+\frac{1}{2} \Delta t
$$

Note, if one introduces the non-dimensional LBM kinematic viscosity $\nu^{\prime}=\nu \tau / \lambda^{2}$ and speed of sound $c_{s}^{\prime}=c^{\prime} / \sqrt{3}=1 / \sqrt{3}$, Eq. 1.35 yields the standard LBM relaxation time, $\tau_{g}^{\prime}=\nu^{\prime} / c_{s}^{\prime 2}+\Delta t^{\prime} / 2=3 \nu^{\prime}+1 / 2$.

\section{Correction of velocity field based on a Poisson equation}

Due to the absence of a pressure gradient term in the modified NS Eq. (1.33), the velocity field, $\boldsymbol{u}^{*}$, which is calculated at every time step with Eq. (1.26) based on the modified distribution functions, computed as a function of time with Eq. 1.34 in the LBM, is only an approximation of the actual velocity field $\boldsymbol{u}$. One additional step is thus required to both compute the pressure field and a corresponding correction $\Delta \boldsymbol{u}$ of the velocity, in order to satisfy the full NS Eqs. (1.14) and (1.15). Following Inamuro et al. [10] we define,

$$
\boldsymbol{u}=\boldsymbol{u}^{*}+\Delta \boldsymbol{u} \quad \text { with } \quad \Delta \boldsymbol{u} \simeq-\Delta t \frac{\nabla p}{\rho} .
$$

Thus, for the actual velocity field to satisfy continuity equation (i.e., $\nabla . \boldsymbol{u}=0$, assuming an incompressible fluid), $\boldsymbol{u}^{*}$ must satisfy the following Poisson equation, 


$$
\nabla \cdot\left(\frac{\Delta t \nabla p}{\rho}\right)=\nabla \cdot \boldsymbol{u}^{*}
$$

This Poisson equation 1.37 could be discretized and solved by various methods. Here, we iteratively solve it in the LBM framework, using the following evolution equation and a second set of particle distribution functions $h_{i}(i=0, \ldots, 8)$,

$$
h_{i}^{n}\left(\boldsymbol{x}+\boldsymbol{e}_{i} \Delta t, t+\Delta t\right)=h_{i}^{n}(\boldsymbol{x}, t)-\frac{\Delta t}{\tau_{h}}\left(h_{i}^{n}-h_{i}^{(e q, n)}\right)-\Delta t w_{i}\left(\nabla \cdot \boldsymbol{u}^{*}(t)\right),
$$

where $n$ denotes the $n$-th iteration in the solution. The equilibrium distribution functions are simply defined as,

$$
h_{i}^{(e q, n)}=\frac{w_{i} p^{n}(\boldsymbol{x}, t)}{\rho_{0} c^{2}},
$$

where $\rho_{0}$ is a reference density. The relaxation time $\tau_{h}$ is related to the density by,

$$
\tau_{h}=\Delta t\left\{\frac{1}{2}+\frac{\rho_{0} c^{2}}{\rho c_{s}^{2}}\right\}
$$

or in LBM variables, $\tau_{h}^{\prime}=1 / 2+\rho_{0}^{\prime} / \rho^{\prime}$, with $\rho^{\prime}=\rho \lambda^{3} / \varpi$ and $\rho_{0}^{\prime}=\rho_{0} \lambda^{3} / \varpi$. In this LBM scheme, pressure is simply calculated as the zero-th order moment of the particle distribution functions as,

$$
p^{n+1}=\rho_{0} c^{2} \sum_{i=0}^{b} h_{i}^{n} .
$$

This scheme is iteratively run at a given time $t$, until the pressure field converges to a stable solution. Once this is achieved, the correction to the velocity field is calculated using Eq. (1.36). In our new method, the two previously derived LBM schemes thus solve, at time $t$ : (i) the pressureless NS equations for high density ratios, with surface tension forces partly included in the formulation of the equilibrium distribution functions and in the body forces, which yields $\boldsymbol{u}^{*}$; and (ii) a Poisson equation using the approximate velocity field as an equivalent "volume 
force" to account for pressure gradients, which yields $\boldsymbol{u}$ and $p$. By contrast with sharp interface methods, the calculation of the interface curvature is not necessary in this method, but only the gradients of the phase field function $\phi$ are needed.

\subsubsection{LBM for solving Cahn-Hilliard equation}

The diffusive interface motion is modeled by the Cahn-Hiliard Eq. (1.12), where the left hand side describes the interface advection, and the right hand side the diffusive transport; $M$ denotes the mobility and the chemical potential $\mu_{\phi}$ is defined by Eq. (1.3), as a function of the bulk free-energy density $\Psi$ and the phase field parameter $\phi$. To solve this equation, we also use an LBM and introduce a third set of probability distribution functions, $f_{i}(\boldsymbol{x}, t)$, whose evolution is again governed by a standard LBM scheme,

$$
f_{i}\left(\boldsymbol{x}+\boldsymbol{e}_{i} \Delta t, t+\Delta t\right)=f_{i}(\boldsymbol{x}, t)-\frac{\Delta t}{\tau_{f}}\left(f_{i}(\boldsymbol{x}, t)-f_{i}^{(e q)}(\boldsymbol{x}, t)\right)
$$

This formulation also uses the SRT collision operator where, by analogy with Swift et al. 23], we define the moments of $f_{i}$ to be the phase field parameter, its flux, and a higher-order moment, respectively, as,

$$
\begin{aligned}
& \sum_{i=0}^{b} f_{i}=\phi \\
& \sum_{i=0}^{b} f_{i} e_{i \alpha}=\phi u_{\alpha}, \\
& \sum_{i=0}^{b} f_{i} e_{i \alpha} e_{i \beta}=\frac{M \mu_{\phi} \delta_{\alpha \beta}}{\tau_{f}-\frac{1}{2} \Delta t}+\phi u_{\alpha} u_{\beta} .
\end{aligned}
$$

The equilibrium distribution functions for $f_{i}(\boldsymbol{x}, t)$ are further defined as,

$$
f_{i}^{(e q)}=H_{i} \phi+v_{i} \frac{M}{\tau_{f}-\frac{1}{2} \Delta t} \mu_{\phi}+\phi w_{i}\left\{\frac{e_{i \alpha} u_{\alpha}}{c_{s}^{2}}+\frac{\left(e_{i \alpha} u_{\alpha}\right)^{2}}{2 c_{s}^{4}}-\frac{|\boldsymbol{u}|^{2}}{2 c_{s}^{2}}\right\},
$$

where $w_{i}$ and $v_{i}$ are the weighing functions defined in Eqs. (1.29) and (1.30), and,

$$
H_{0}=1, H_{i}=0 ;(i=1,2, \ldots, 8) .
$$


It can be shown by Chapman-Enskog expansion that with these definitions, the LBM scheme solves the $\mathrm{CH}$ convection-diffusion equation.

Density $\rho$ is assumed to vary smoothly across the two-fluid interface and is calculated throughout the LBM domain as a function of the order parameter $\phi$, as,

$$
\rho(\phi)= \begin{cases}\rho_{2} & \phi \leq \phi_{2} \\ \frac{\phi-\phi_{2}}{\phi_{1}-\phi_{2}}\left(\rho_{1}-\rho_{2}\right)+\rho_{2} & \phi_{2}<\phi<\phi_{1} \\ \rho_{1} & \phi \geq \phi_{1}\end{cases}
$$

Similarly, both kinematic and dynamic viscosities are calculated as a function of density as,

$$
\begin{aligned}
& \nu(\rho)=\frac{\rho-\rho_{2}}{\rho_{1}-\rho_{2}}\left(\nu_{1}-\nu_{2}\right)+\nu_{2}, \\
& \mu(\rho)=\frac{\rho-\rho_{2}}{\rho_{1}-\rho_{2}}\left(\mu_{1}-\mu_{2}\right)+\mu_{2} .
\end{aligned}
$$

respectively.

\subsubsection{Boundary conditions}

Wall boundary conditions are introduced here, for the three LBM distribution functions $f_{i}, g_{i}$, and $h_{i}$.

For $f_{i}$ and $g_{i}$, which are used for the fluid momentum and interface tracking equations, Eqs. (1.23) and (1.44) indicate that the first-order moment is related to the macroscopic velocity, as in standard LBM approaches. Thus, for no-slip boundary conditions along solid walls, velocities are zero and hence the unknown particle distribution functions can be obtained from standard LBM bounce back schemes. In those, particles are specified to reflect back off the wall, into the fluid domain, resulting in a zero fluid velocity at the wall surface [20].

For the boundaries with periodic conditions, the unknown particle distribution functions on one boundary are set equal to the particle distribution functions on the other boundary, where the periodicity condition has been implemented [20]. 
At a stationary wall, the boundary condition of distribution functions $h_{i}$, used in the LBM solution of the pressure Poisson equation, follows from the NS momentum equation. For instance, assuming that the wall is planar and perpendicular to gravity $\boldsymbol{g}=-g \boldsymbol{j}$, Eqs. (1.14), 1.15) and (1.16) yield,

$$
\frac{\partial p}{\partial y}=-\rho g+\mu\left(\frac{\partial^{2} v}{\partial x^{2}}+\frac{\partial^{2} v}{\partial y^{2}}\right)
$$

An approximation of the pressure at the wall $\left(x=x_{w}\right)$ as a function of its values at neighboring lattice points can then be obtained from a Taylor series expansion in the direction perpendicular to the wall as,

$$
p\left(x_{w}, y_{w}, t\right)=p\left(x_{w}, y_{w}-\Delta x, t\right)-\frac{\partial p}{\partial y}\left(x_{w}, y_{w}, t\right) \Delta x+\mathcal{O}\left(\Delta x^{2}\right),
$$

where we can substitute the pressure gradient from its value in Eq. (1.51). Based on this equation, boundary values of the unknown particle distribution functions $h_{i}$ are finally specified at the wall by assuming these are equal to the equilibrium

distribution functions [24], $h_{i}\left(x_{w}, t\right)=h_{i}^{(e q)}\left(p\left(x_{w}, t\right)\right)$, calculated with Eq. 1.39. For more complex geometries, same procedure can be done by finding the pressure value on the neighbour lattice node to the wall. compute the pressure on the wall bye a Taylor series and then use Eq. (1.39) to find unknown distribution functions.

\subsubsection{Computation of spatial derivatives}

The first and second spatial derivatives in the various LBM equations defined above (e.g., Eqs. (1.3), 1.28), 1.31), 1.34) and (1.36) ) are computed using the following centered finite difference schemes, that are typical of standard LBM implementations [9], i.e., for the D2Q9 scheme and $\alpha=1,2$,

$$
\frac{\partial \Phi}{\partial x_{\alpha}}(\boldsymbol{x})=\sum_{i=1}^{9} w_{i} e_{i \alpha} \frac{\Phi\left(\boldsymbol{x}+\boldsymbol{e}_{i} \Delta t\right)-\Phi\left(\boldsymbol{x}-\boldsymbol{e}_{i} \Delta t\right)}{2 c_{s}^{2} \Delta t}+\mathcal{O}\left(\Delta x^{2}\right),
$$

and,

$$
\frac{\partial^{2} \Phi}{\partial x_{\alpha}^{2}}(\boldsymbol{x})=\sum_{i=1}^{9} w_{i} \frac{\Phi\left(\boldsymbol{x}+\boldsymbol{e}_{i} \Delta t\right)-2 \Phi(\boldsymbol{x})+\Phi\left(\boldsymbol{x}-\boldsymbol{e}_{i} \Delta t\right)}{c_{s}^{2} \Delta t^{2}}+\mathcal{O}\left(\Delta x^{2}\right) .
$$


for an LBM cell of coordinate $\boldsymbol{x}$, with $\Phi$ denoting any of relevant flow parameter and $w_{i}$ the weight factors defined in Eq. (1.29). Along the boundary, except when a periodicity condition is specified, we use first-order de-centered finite difference schemes.

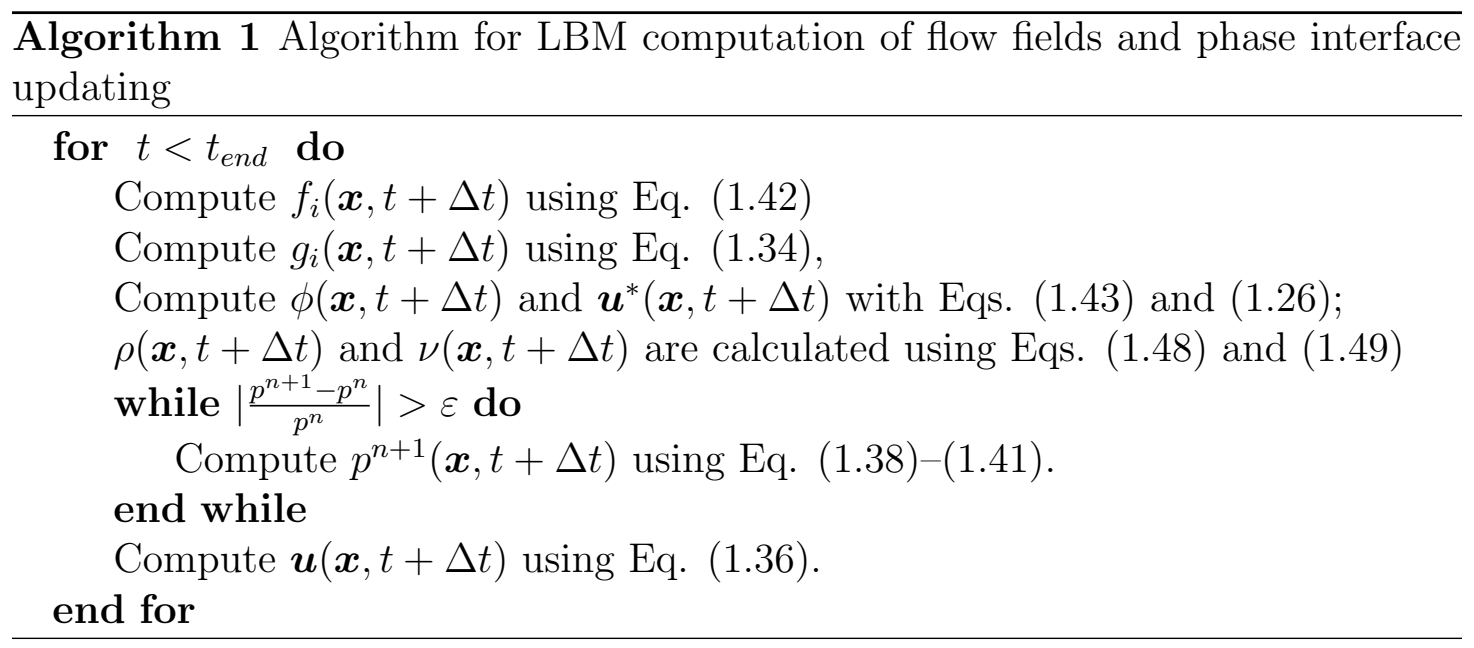

\subsubsection{Summary of LBM algorithm}

The resulting LBM algorithm for the calculation of $2 \mathrm{D}$ flows of two fluids having a high density ratio, and the updating of the phase interface is summarized in 1 .

As indicated in the introduction, although we built our work in part based on Inamuro et al.'s [10] results, there are significant differences between our approach and theirs. These are summarized in the following. First of all, in their work, they solve the following equation for the interface tracking,

$$
\frac{\partial \phi}{\partial t}+\frac{\partial\left(\phi u_{\alpha}\right)}{\partial x_{\alpha}}=\Theta \frac{\partial^{2} P_{\alpha \beta}}{\partial x_{\alpha} x_{\beta}}
$$

where $\Theta$ is a diffusion coefficient and $P_{\alpha \beta}$ is defined as,

$$
P_{\alpha \beta}=\left[\phi \frac{\partial \Psi}{\partial \phi}-\Psi-k_{f} \phi \frac{\partial^{2} \phi}{\partial x_{\gamma} \partial x_{\gamma}}-\frac{k_{f}}{2} \frac{\partial \phi}{\partial x_{\gamma}} \frac{\partial \phi}{\partial x_{\gamma}}\right] \delta_{\alpha \beta}
$$

By comparing this equation with the Cahn-Hilliard Eq. 1.12 used in our model, we see that the right hand sides of each equation are different and, unlike in 
our case, Inamuro et al. did not provide a clear physical interpretation for their equation.

Second, Inamuro et al. defined three separate variables $k_{f}, k_{g}$ and $T$ for calculating surface tension and interface thickness. As showed before, in our method, only two different coefficients $k$ and $\beta$ are used, which can be expressed as a function of interface thickness $W$ and the surface tension coefficient $\sigma_{12}$, using Eqs. (1.7) and (1.11).

Third, in Inamuro et al.'s scheme, the particle distribution functions $g_{i}$ 's used for calculating the hydrodynamic fields are just solving the advection part of the (NS) momentum equation. The effect of the viscous stress tensor is implemented by adding an extra term to the collision part of the $g_{i}$ equation (corresponding to our Eq. (1.21)), and the viscosity effects resulting from this extra term have no clear physical interpretation. By contrast, in our model, dynamic viscosity is rigorously related to relaxation time in a way that is consistent with classical LBM schemes.

Finally, and importantly, unlike Inamuro et al.'s code, our LBM scheme is fully optimized and implemented as a highly efficient parallel code on a GPGPU hardware, as summarized in section 1.4 .

\subsection{GPGPU Implementation of the LBM code}

GPGPUs are computing hardware with a large number of cores (448 on the nVIDIA Tesla C2070), and a shared memory (6MB for the nVIDIA Tesla C2070), that execute a number of computing threads in parallel. To manage these threads, the CUDA programming environment offers two levels of parallelism. First, all the threads are grouped in one thread block where extremely fast memory is shared between the threads, which can be synchronized. Each thread is identified by its three-dimensional thread index, which gives the position in the thread block 
within the hardware. To efficiently use the hardware, the total number of threads per block should be in the range of 64 to 512. This number can be adjusted up or down depending on the size of local and shared memory available on each particular GPGPU. Threads are executed in warps containing 32 threads each, on one of the GPGPU multiprocessors. Second, the thread blocks are bundled into the grid. Unlike threads located within the same thread block, threads in different blocks can only communicate via the GPGPU shared memory and a synchronization is not possible. Blocks are identified by their two-dimensional block index, namely their position within the grid. Further details on the thread processing, grouping in warps, and distribution among the GPGPU multiprocessors can be found in [25].

\subsubsection{Topology and grid mapping}

The main design element in the GPGPU implementation of a numerical method is the mapping of the numerical grid onto the computational hardware, i.e., in our case the mapping of LBM nodes onto the GPGPU processors, blocks, and threads. In earlier GPGPU hardwares, several restrictions existed on memory access patterns that needed to be taken into account in order to achieve maximum performance (see, e.g., [12]). However, recent GPGPUs dedicated to numerical computations offer higher flexibility, so that in this model we decided not to use the earlier shared memory particle propagation pattern but instead to access the GPGPU main memory directly in the propagation step. Hence, by contrast with earlier implementations, the thread blocks can be designed almost arbitrarily.

Specifically, in our grid mapping, we assign one single lattice node to one CUDA thread. The memory is allocated as a one-dimensional array and, as proposed by [16], the memory index is calculated as $\mathrm{k}=\mathrm{nx} * \mathrm{y}+\mathrm{x}$, for a node at position $(x, y)$ and a total of $n_{x} \times n_{y}$ nodes. The dimension of the grid and the 
number of threads are specified in the CUDA kernel call and the coordinates of an LBM node can then be determined via, $\mathrm{x}=$ threadId. $\mathrm{x}$, and $\mathrm{y}=$ blockIdx. $\mathrm{x}$. After the kernel launch, CUDA manages the exact distribution of tasks among the multiprocessors and cores on the GPGPU.

\subsubsection{Implementation details}

The implementation of most of the LBM kernels is straightforward, as demonstrated in [16]. The CUDA interface supports C-style programming, so that standard $\mathrm{C}$ codes written for single processors can be easily transferred. Note that all the computations in this work require double precision variables to ensure accuracy and convergence. On the latest nVIDIA boards, double precision computations are only a factor of 2 slower than single precision ones. Due to the doubled memory requirement of double precision, memory transfers are also a factor of 2 slower, so that for our LBM algorithm, we can approximately estimate that the performance in double precision is half that of single precision.

In general, the performance of our proposed LBM multiphase model highly depends on the number of Poisson iterations performed at each time step, which depends on the problem physics. Hence no generally valid performance value of the multiphase scheme can be given and performance must instead be assessed on a case-by-case basis. Performance details are given below for the applications presented in the validation section.

\section{Memory allocation}

In GPGPU implementations, data transfer between the host (i.e., the CPU computer controlling the GPGPU hardware) and GPGPU memory, usually significantly penalizes performance and hence must be minimized. To do so, in this LBM, unlike in previous implementations, we do not allocate host memory for the 
full 3 sets of particle distribution functions (PDFs), but instead these are only allocated on the GPGPU. Hence, in the post-processing step, which involves data transfer from the GPGPU to the host, only the macroscopic values, such as pressure, velocity and phase field parameter (i.e., 3-5 double precision variables) are copied to the host memory, instead of copying the full sets of PDFs.

Additionally, in GPGPUs, the memory is accessed as one single vector (with the limitation in CUDA $\mathrm{C}$ codes that the function parameter space of the kernel calls be less than 256 bytes). In a D2Q9 double precision model, $2 \times 9 \times 8=144$ bytes are needed for pointers to the GPGPU memory, to refer to the kernels where data is located. To reduce the number of pointers, the memory for all PDFs is allocated at once, leading to a single linear memory segment. The individual memory locations are then computed within each thread. Hence, as the data layout structure is clearly defined, it is sufficient to only send the start address of the PDF arrays to the kernels. This saves function parameter space, which can thus be used for storing pointers to other variables, such as the macroscopic variables, derivatives, and so on.

\section{Boundary conditions}

Boundary conditions (BCs) disturb the flow of the LBM algorithm, as they require additional operations on a specific subset of nodes; hence this affects model performance. In general, a single LB kernel for all lattice nodes is preferable on a data- and thread-parallel system, for optimal load balancing. However, this is not possible for all the boundary conditions used in this work, which require additional kernel launches to process the particle distribution functions. To optimize parallel LBM computations, in our model, ghost layers of lattice nodes surround the whole computational domain, so that all particle distribution functions can be advected to neighboring nodes (propagation step), even at the domain boundary; this way, 
no logical test is required inside the LBM kernels regarding boundaries. Then, after the standard kernels for collision and propagation have been run, BCs are applied. No-slip BCs for instance are simply specified by bouncing the PDFs, that have been advected into the ghost layer, back into the domain (bounce-back scheme). In extrapolation (or open) boundary conditions, values from the next-to-last fluid node are copied to the last one. Here, the problem of thread synchronization leads to a second justification for using separate BC kernels and launching them after the calculation of the flow field is complete. Such BCs indeed rely on consistent and valid particle distribution functions at the neighboring lattice nodes, so that these nodes need to have terminated their flow field updating before the BCs can be applied. In general, we found that the additional computational overhead related to the BC kernel launches is more than compensated by a higher flexibility in setting up BCs in the model (i.e., allowing to easily switch boundary conditions), and an easier model upgrade (i.e., implementation of further boundary condition types without having to modify the basic LB kernels).

\section{Convergence check for Poisson iteration}

A loop over all lattice nodes is needed to evaluate the maximum error during iterations in the solution of the pressure Poisson equation. To improve performance, this error is only computed on the GPGPU without copying all node results back to the host memory. However, such operations in thread-parallel systems require a careful treatment to avoid "race conditions" among threads, which would lead to inaccuracies. Thus, a first kernel computes the maximum error in one thread block, where local errors are calculated for each computational node and the maximum error in the block is then calculated in a "divide and conquer" strategy, with the help of the thread-global shared memory and thread block synchronization points. Maximum errors of each thread block are stored in the GPGPU memory (as a vec- 
tor of $n_{y}$ values), and the same steps are applied to calculate the global maximum error among blocks, which is finally copied to the memory of the CPU host. The latter is responsible for kernel flow control and stopping the Poisson iterations, once a certain relative error threshold $\varepsilon$ is reached. It should be mentioned that Poisson solution convergence is not necessary to be checked after each iteration. The relative error is checked every 20 iteration to improve the performance of the computations.

\section{Computational algorithm}

The GPGPU computational algorithm is summarized in 2 , including the previously mentioned approach for boundary conditions and the Poisson iteration convergence check. Moreover, additional kernels are introduced for the calculation of derivatives, such as the divergence of the predicted (pressureless) flow field $\nabla \cdot \boldsymbol{u}^{*}$. Before the time loop starts, several iterations of the phase field kernel (with a zero velocity field) are run, to let the interface between the two fluids converge to an initial equilibrium diffusive interface shape. This is of great importance when no analytical solution for the initial interface shape exists.

\subsubsection{Performance}

As indicated before, the performance of our LBM multiphase model highly depends on the number of Poisson iterations performed at each time step, which depends on the problem physics. Hence performance is assessed on a case-by-case basis for each case presented in the application Section 5. Thus, Table (1.1) lists the average value of "Million Node Updates Per Second" (MNUPS) achieved for the different test cases. We see that the best performance (52) is achieved in the first case, which does not require solving the pressure Poisson equation, while the other cases, which require such a solution have their performance reduced by a factor of 


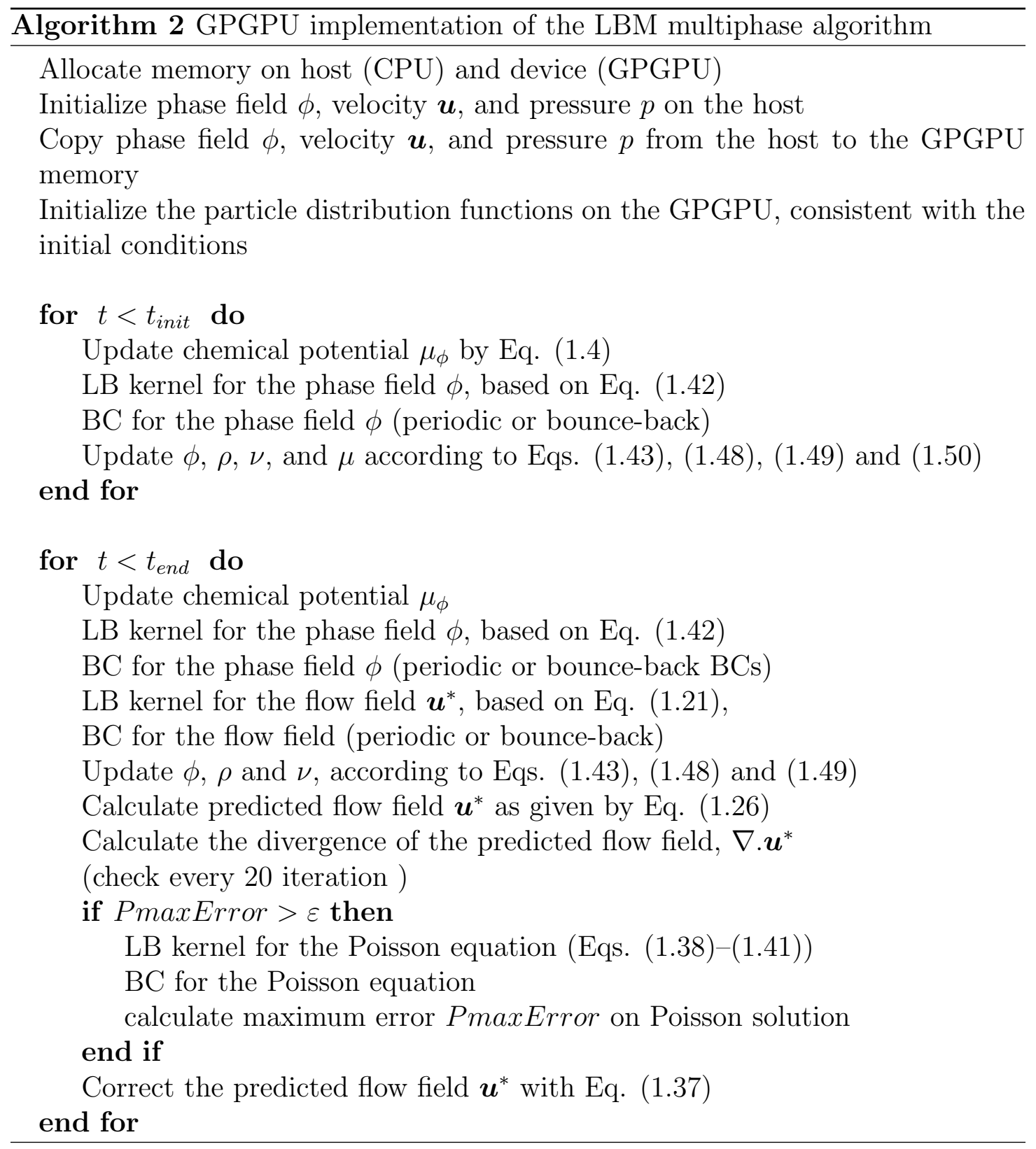


5-6. For comparison, a FORTRAN single-processor (CPU) implementation done earlier for a similar code achieved MNUPS values about 40 times smaller than on the GPGPU.

\begin{tabular}{c||c}
\hline Case & MNDUPS \\
\hline Two-fluid Poiseuille flow & 52 \\
Stationary bubble & 12 \\
Rising bubble (case (a)) & 9.6 \\
Rising bubble (case (b)) & 8.4 \\
Rising bubble (case (c)) & 8.2 \\
Rayleigh-Taylor instability & 11 \\
Breaking wave & 8.1 \\
\hline
\end{tabular}

Table 1.1: Performance for different test cases presented in the application section

\subsection{Applications}

Here, we present applications that validate our newly developed LBM for multiphase flows, by comparing numerical results to the solution of analytical and experimental benchmark problems. First, the LBM scheme is applied to simulating a two-fluid laminar Poiseuille flow between infinite plates, for which there is an analytical solution; this assesses the method's accuracy in the absence of surface tension effects (since the fluid interface curvature is zero) in case of large density gradients. Second, we solve the case of a stationary bubble of a lighter fluid in a non-moving heavier fluid, in the absence of gravity; this validates the computation of surface tension effects, by comparison with Laplace's law. Then, we model a lighter fluid bubble rising in a heavier stationary fluid, and compare LBM results to other numerical results, which provide an independent reference simulation. Finally, we apply the LBM model to more complex two-fluid flows with a large density ratio: (i) a Rayleigh-Taylor instability; and (ii) ocean wave breaking. 


\subsubsection{Definitions}

In all the following simulations, values are provided for non-dimensional lattice variables (denoted by a prime), which are the parameters actually used in LBM computations. Besides having $\Delta x^{\prime}=\Delta t^{\prime}=c^{\prime}=1$, as stated before, in all cases the non-dimensional relaxation time for solving the momentum equations is kept within the limits, $0.5<\tau_{g}^{\prime} \leq 1$, in order to ensure stability of the LBM solution [20]. With Eq. (1.35), this requires $0<\nu^{\prime} \leq 1 / 6$, and hence the time step is defined as, $\Delta t=(\Delta x)^{2} \nu^{\prime} / \nu \leq(\Delta x)^{2} /(6 \nu)$. The non-dimensional surface tension coefficient is further defined as,

$$
\sigma_{12}^{\prime}=\frac{\sigma_{12} \tau^{2}}{\rho_{0} \lambda^{3}}=\frac{\sigma_{12}}{\rho_{0} c^{2} \Delta x}
$$

The non-dimensional relaxation time for solving the Cahn-Hilliard Eq. (1.42) is set to $\tau_{f}^{\prime}=\tau_{f} / \Delta t=1$. The non-dimensional mobility $M^{\prime}=M \varpi /\left(\tau \lambda^{3}\right)$ is set to $M^{\prime}=0.02 / \beta^{\prime}$ to get the most stable results [27]; additionally, we specify $\phi_{1}=0.4$ and $\phi_{2}=0.1$ in all cases, and the interface thickness is assumed to be of 4 lattice meshes: $W=4 \Delta x$ or $W^{\prime}=4$, which by combining Eqs. 11.7) and (1.11) yields the interface lattice parameters,

$$
\beta^{\prime}=\frac{3 \sigma_{12}^{\prime}}{\left(\phi_{1}-\phi_{2}\right)^{4}} \quad \text { and } \quad k^{\prime}=\frac{6 \sigma_{12}^{\prime}}{\left(\phi_{1}-\phi_{2}\right)^{2}}
$$

where $\beta^{\prime}=\beta /\left(\rho_{0} c^{2}\right)$ and $k^{\prime}=k /\left(\rho_{0} c^{2} \lambda^{2}\right)$. Although other values of $W$ (both smaller and larger) were tested in applications, the selected interface thickness was found to yield the most accurate results in applications, as compared to reference results, by ensuring a sufficient sharpness of the interface gradients while not causing numerical instabilities with unnecessary large gradients.

The non-dimensional relaxation time used for solving the pressure Poisson Eq. (1.38) is similarly kept within the range $0.5<\tau_{h}^{\prime} \leq 1$ [24]; with the latter constraint, Eq. 1.40 yields that for either fluid the non-dimensional density 
$\left(\rho_{1}^{\prime}, \rho_{2}^{\prime}\right) \geq 6$. Finally, the non-dimensional reference density is set to $\rho_{0}^{\prime}=1$, which implies that, $\varpi=\rho_{0}(\Delta x)^{3}$ (usually, one will also assume for simplicity, $\rho_{0}=1$ $\left.\mathrm{kg} / \mathrm{m}^{3}\right)$ and $\rho_{i}^{\prime}=\rho_{i} / \rho_{0}$, for fluid $i=1,2$.

\subsubsection{Two-fluid Poiseuille flow}

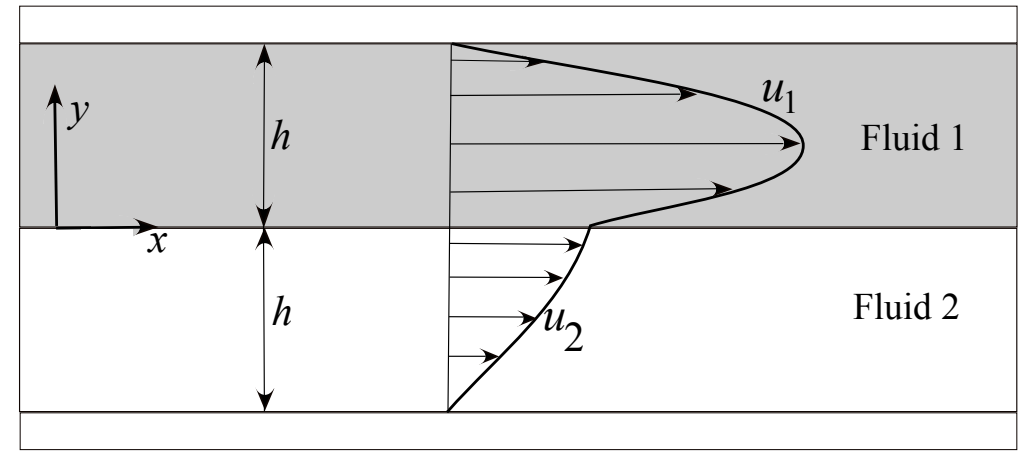

Figure 1.2: Definition sketch of a two-fluid Poiseuille flow between infinite plates, with typical analytical solution for the horizontal velocity profile $u(y)$.

The two-fluid Poiseuille flow, between two infinite plates, inclined at an angle $\alpha$ with respect to the horizontal, is a good analytical test case to validate the method for both high fluid dynamic viscosity and density ratios in the absence of surface tension effects. Two immiscible fluids are accelerated in between the plates by a body force (i.e., projected gravity, $\rho g \sin \alpha$ ) and slowed by the viscous shear along the plate surfaces (Fig. 1.2). At the planar two-fluid interface, the continuity of fluid velocity and stresses has to be satisfied and, in such a case, geometry also implies that surface tensions forces are zero. An analytical solution of the steady state NS equations can be derived for this case, to which the fully developed velocity field computed by the LBM can be compared.

Specifically, the analytical reference solution is derived by solving the following simplified, but exact, one-dimensional momentum equation for the horizontal 
velocity component $u_{i}$ in each fluid $(i=1,2)$ (with $\left.y=x_{2}\right)$,

$$
-\rho g \sin \alpha=\mu \frac{d^{2} u_{i}}{d y^{2}}
$$

which yields [28],

$$
\begin{aligned}
& u_{1}=\frac{g \sin \alpha}{2}\left\{h^{2} \frac{\left(\rho_{1}+\rho_{2}\right)}{\left(\mu_{1}+\mu_{2}\right)}-y h \frac{\left(\mu_{1} \rho_{2}-\mu_{2} \rho_{1}\right)}{\mu_{1}\left(\mu_{1}+\mu_{2}\right)}-y^{2} \frac{\rho_{1}}{\mu_{1}}\right\}, \\
& u_{2}=\frac{g \sin \alpha}{2}\left\{h^{2} \frac{\left(\rho_{1}+\rho_{2}\right)}{\left(\mu_{1}+\mu_{2}\right)}-y h \frac{\left(\mu_{1} \rho_{2}-\mu_{2} \rho_{1}\right)}{\mu_{2}\left(\mu_{1}+\mu_{2}\right)}-y^{2} \frac{\rho_{2}}{\mu_{2}}\right\} .
\end{aligned}
$$

with fluid densities $\rho_{1}$ and $\rho_{2}$, and dynamic viscosities $\mu_{1}$ and $\mu_{2}$, respectively; $g$ denotes the gravitational acceleration and $2 h$ is the distance between the 2 plates. This analytical solution yields velocity profiles that are parabolic in each fluid, with a discontinuity of the vertical velocity gradient at the interface, owing to the identical horizontal stress on either side of the interface, for different viscosity values (Fig. 1.2).

LBM simulations are started from a state of rest in a $2 \mathrm{D}$ channel similar to that sketched in Fig. 1.2. A periodic boundary condition is specified for lateral upstream and downstream boundaries, in the flow direction $x=x_{1}$, and no-slip boundary conditions are specified on the plate surfaces $(y= \pm h)$. The interface parameters of the system are set to $k^{\prime}=0.01$ and $\beta^{\prime}=0.05$. Thanks to the periodicity in flow direction and the laminar nature of the flow, the grid resolution in the $x$ direction can be low, and only 4 grid points were used. The number of grid points in the vertical $y$ direction is $N=2 h^{\prime}=100$ to 300 , with $\Delta x=2 h / N$ (Table 1.2).

A convergence study of LB results accuracy as a function of mesh size $N$ is performed for a series of flows defined by a constant Reynolds number, $\operatorname{Re}=u_{1}^{\max } 2 h / \nu_{1}=100$ (based on maximum velocity in the channel, assuming this occurs in fluid 1, corresponding kinematic viscosity, and channel width); the LBM Mach number in these computations is also fixed at, $\mathrm{Ma}=u_{1}^{\max } / c_{s}=0.01$, 
which provides sound speed $c_{s}$ to use for a given flow. For each flow parameters and LBM discretization $N$ or $h^{\prime}$, the non-dimensional body force magnitude, $g^{\prime}=g \sin \alpha\left(\tau^{2} / \lambda\right)=g \sin \alpha \Delta t / c^{2}$ (with $\left.c^{2}=3 c_{s}^{2}\right)$ is adjusted by varying the channel angle $\alpha$, in order for the maximum flow velocity $u_{1}^{\max }$ (obtained from the analytical solution) to satisfy the desired Reynolds number. In practice, given fluid and geometry parameters $\rho_{1} / \rho_{2}, \nu_{1} / \nu_{2}, h$ and $g$ and combining the expression for $u_{1}^{\max }$ with the definitions of Re and Ma, we find $g^{\prime}$ as a function of $h^{\prime}$ (Table 1.2 ).

Let us first assume, the density of fluid phase 1 is $\rho_{1}^{\prime}=600$ and the density ratio is $\rho_{1}^{\prime} / \rho_{2}^{\prime}=100$ (thus $\rho_{2}^{\prime}=6$ ); the kinematic viscosity ratio is $\nu_{1} / \nu_{2}=0.1$, which yields $\mu_{1} / \mu_{2}=10$. To check the accuracy of numerical results, we calculate the $L_{2^{-}}$ norm relative error (i.e., a RMS error between numerical and analytical velocities $u$, scaled by the RMS of the analytical velocity). LBM simulations were stopped when the difference between the $L_{2}$-norm of two consecutive time steps became less than $10^{-6}$. Fig. 1.3 shows the analytical and numerical (steady-state) velocity profiles for different grid sizes $h^{\prime}$, and the convergence to the analytical reference solution is seen to be quite good. In previous studies of two-phase Poiseuille flows with LBM multiphase models [29, 30], numerical oscillations of the fluid velocity were observed near the phase interface, even for low density ratios. Our LBM multiphase method does not trigger such oscillations and captures very well the slope discontinuity of the velocity profile at the phase interface. Table 1.2 summarizes the $L_{2}$-norm errors for different grid configurations. Convergence can clearly be observed. Fig. 1.4 further shows a first-order convergence of the relative error is achieved, as a function of the number of lattice nodes in the $y$ direction.

Simulations are run next for different viscosity and density ratios, using a fixed LBM grid resolution with $N=300$ in the $y$ direction. Flow parameters are, $\mathrm{Re}=1000$ and $\mathrm{Ma}=0.005$. Fig. 1.5 shows steady-state velocity profiles 
for three different cases: (a) the fluid densities are identical $\left(\rho_{1}^{\prime} / \rho_{2}^{\prime}=1\right)$, and the kinematic viscosity ratio is $\nu_{1} / \nu_{2}=0.1$ (which yields different velocity gradients at the phase interface to satisfy the continuity of shear stress); (b) $\rho_{1}^{\prime} / \rho_{2}^{\prime}=100$ and $\nu_{1} / \nu_{2}=1$ (which yields continuous shear stress and a continuous velocity gradient at the phase interface: zero, due to the symmetry); and (c) $\rho_{1}^{\prime} / \rho_{2}^{\prime}=1000$ and $\nu_{1} / \nu_{2}=0.0667$, as for water and air, which are the fluids used in our final applications. The good agreement of numerical and analytical results, even in the latter case, confirms the accuracy of the LBM scheme and its applicability to practical problems such as at an air-sea interface.
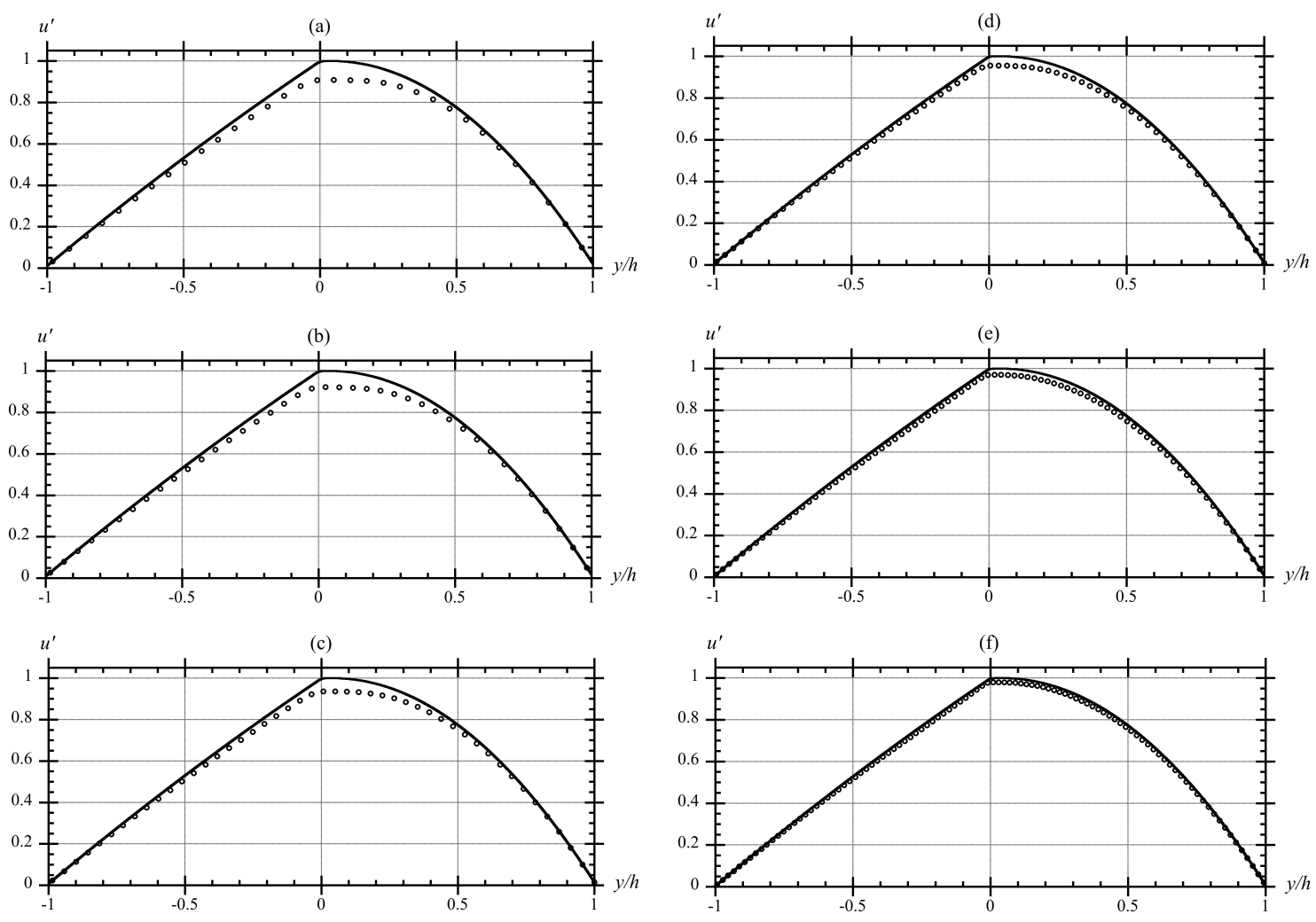

Figure 1.3: Non-dimensional velocity profiles $u^{\prime}=u / u_{1}^{\max }$ in two-fluid Poiseuille flow (Fig. 1.2), for $\mathrm{Re}=100, \mathrm{Ma}=0.01, \rho_{1} / \rho_{2}=100$, and $\nu_{1} / \nu_{2}=0.1:(-)$ analytical; (०) LB results (only $33 \%$ of nodes are plotted for clarity), for different grid resolutions $h^{\prime}$ (see, Table 1.2). 


\begin{tabular}{c||c|c|c}
\hline Case & $h^{\prime}$ & $g^{\prime}$ & $L_{2}$-norm $(\%)$ \\
\hline (a) & 50 & $2.87110^{-08}$ & 6.2 \\
(b) & 60 & $2.39010^{-08}$ & 5.4 \\
(c) & 70 & $2.05110^{-08}$ & 4.4 \\
(d) & 100 & $1.43610^{-08}$ & 3.2 \\
(e) & 125 & $1.14910^{-08}$ & 2.7 \\
(f) & 150 & $9.57210^{-09}$ & 1.9 \\
\hline
\end{tabular}

Table 1.2: $L_{2}$-norm error (between analytical and LB results) and body force magnitude $g^{\prime}$, as a function of LBM discretization size $h^{\prime}$, for the two-fluid Poiseuille flow test case of Fig. 1.3 (see, Fig. 1.4 for error plot).

\subsubsection{Stationary bubble in quiescent fluid}

The exact solution (in the absence of gravity, i.e., volume forces) for a circular stationary bubble of a lighter fluid embedded within a heavier quiescent fluid is used as a benchmark to validate surface tension force computations in our LBM scheme, on the basis of the Cahn-Hilliard equation. Laplace law predicts that, if the two-fluid interface is curved, a pressure jump $\Delta p$ occurs across the interface (with pressure being higher along the concave side); for a two-dimensional circular bubble of radius $R$, we have,

$$
\Delta p=\frac{\sigma_{12}}{R}
$$

A circular droplet of fluid of density $\rho_{2}=10 \mathrm{~kg} / \mathrm{m}^{3}$ and radius $R=0.005 \mathrm{~m}$ is placed in the middle of a square LBM domain with side $d=0.02 \mathrm{~m}$, filled with a fluid of density $\rho_{1}=600 \mathrm{~kg} / \mathrm{m}^{3}$ (hence, the density ratio is $\rho_{1}^{\prime} / \rho_{2}^{\prime}=60$ ). The fluid kinematic viscosities are $\nu_{1}=\nu_{2}=2 \times 10^{-3} \mathrm{~m}^{2} / \mathrm{s}$, and the surface tension coefficient $\sigma_{12}=210^{-3} \mathrm{~N} / \mathrm{m}$. Eq. 1.62 predicts that this situation corresponds to a pressure jump $\Delta p=0.4 \mathrm{~N} / \mathrm{m}^{2}$ across the interface.

In LBM computations, we use all the fixed parameter values specified in the definition section and select a lattice viscosity, $\nu_{1}^{\prime}=\nu_{2}^{\prime}=1 / 6$, i.e., $\tau_{g}^{\prime}=1$. Three grid sizes: $N_{x}=N_{y}=64,128$ and 256 are successively used and periodic boundary conditions are specified on the 4 sides of the square domain. The mesh size is, $\Delta x=$ 


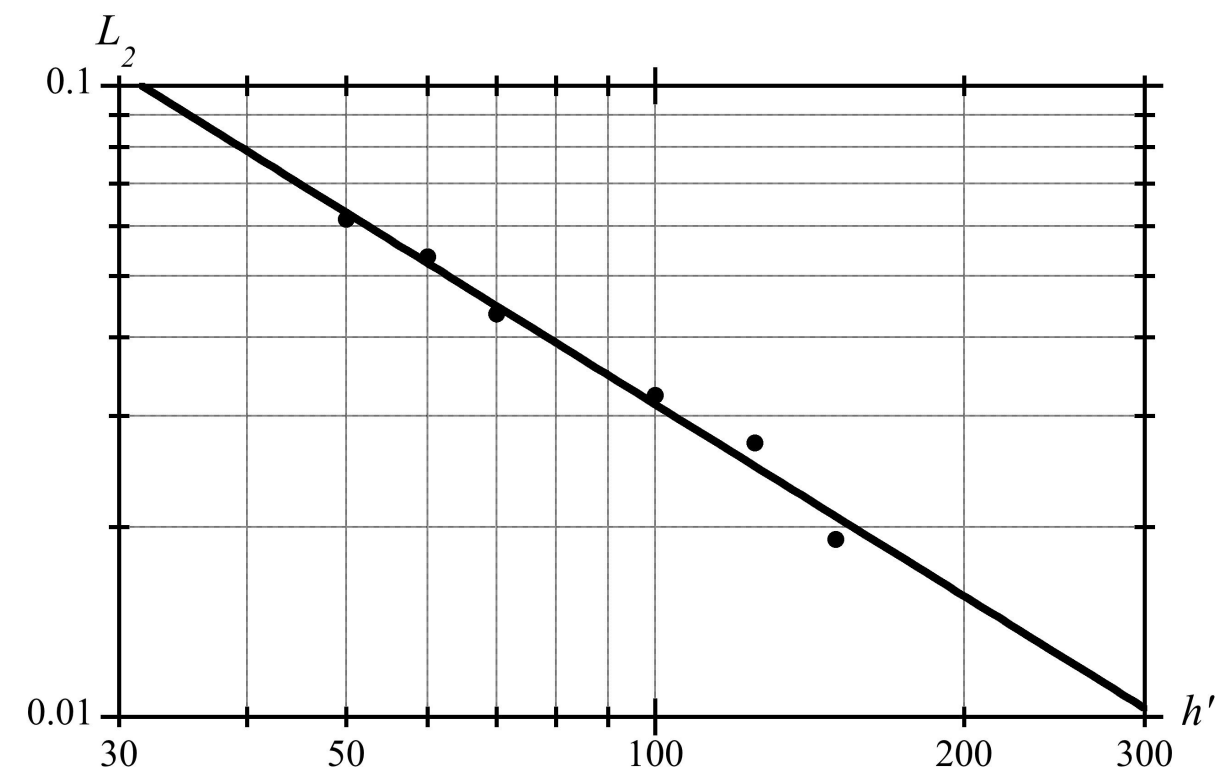

Figure 1.4: $L_{2}$-norm error (between analytical and LB results) as a function of LBM discretization size $h^{\prime}$, for the two-fluid Poiseuille flow test case (Fig. 1.2): $(\bullet)$ data from Table 1.2 $(\longrightarrow)$ power curve fit $L_{2} \propto\left(h^{\prime}\right)^{-1.008}\left(R^{2}=0.99\right)$.

$d / N_{x}$ and time step, $\Delta t=(\Delta x)^{2} /\left(6 \nu_{1}\right)$. The dimensionless lattice surface tension coefficient $\sigma_{12}^{\prime}$ is then given by Eq. (1.57) and the interface lattice parameters $\beta^{\prime}$ and $k^{\prime}$ follow from Eq. (1.58). Table 1.3 lists values of the latter coefficients and gives results for the pressure jump, computed as the difference between the average pressure inside and outside of the droplet, as a function of the discretization size. We see that numerical results are in good agreement with the analytical results, with a $0.72 \% L_{2}$-norm error in the finest discretization. The convergence of numerical errors also appears to be first-order in grid size, as in the previous application.

To further validate the surface tension force computation for a non-stationary case, a similar simulation is repeated for an initially square droplet of a lighter fluid, with side $128 \Delta x$, embedded within the larger square domain of side $d=$ $256 \Delta x=0.02 \mathrm{~m}$ used earlier $\left(\Delta x=7.81310^{-5} \mathrm{~m}\right)$, filled with a heavier fluid. The LBM simulation parameters are, $\rho_{1}^{\prime}=600, \rho_{1} / \rho_{2}=100, \nu_{1}=\nu_{2}=210^{-3} \mathrm{~m}^{2} / \mathrm{s}$, 


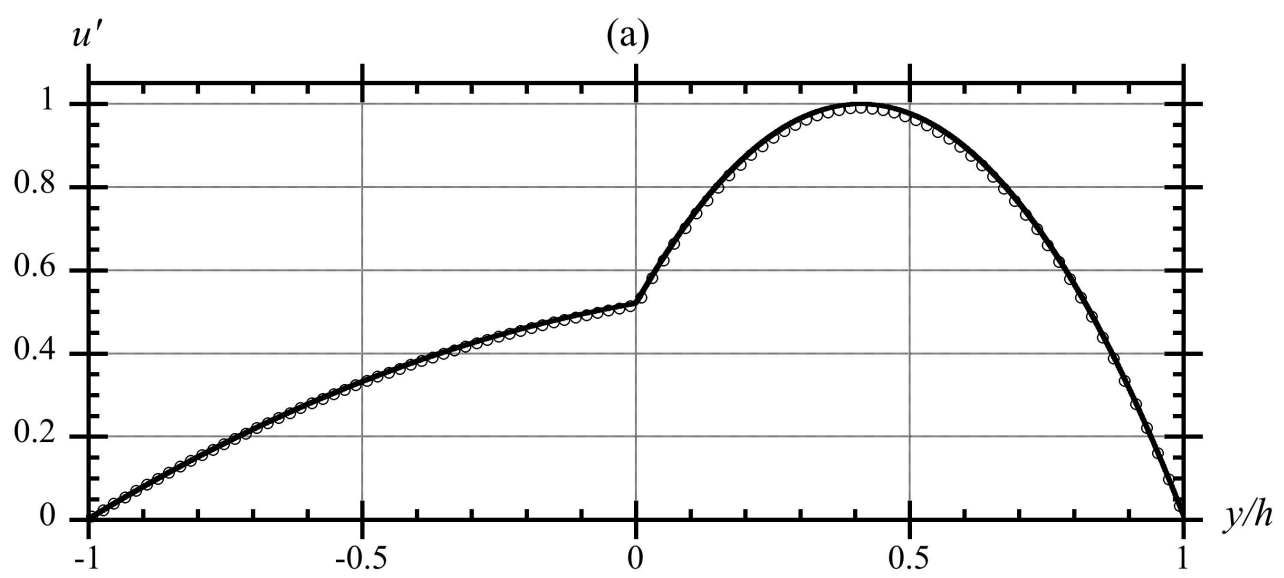

(b)
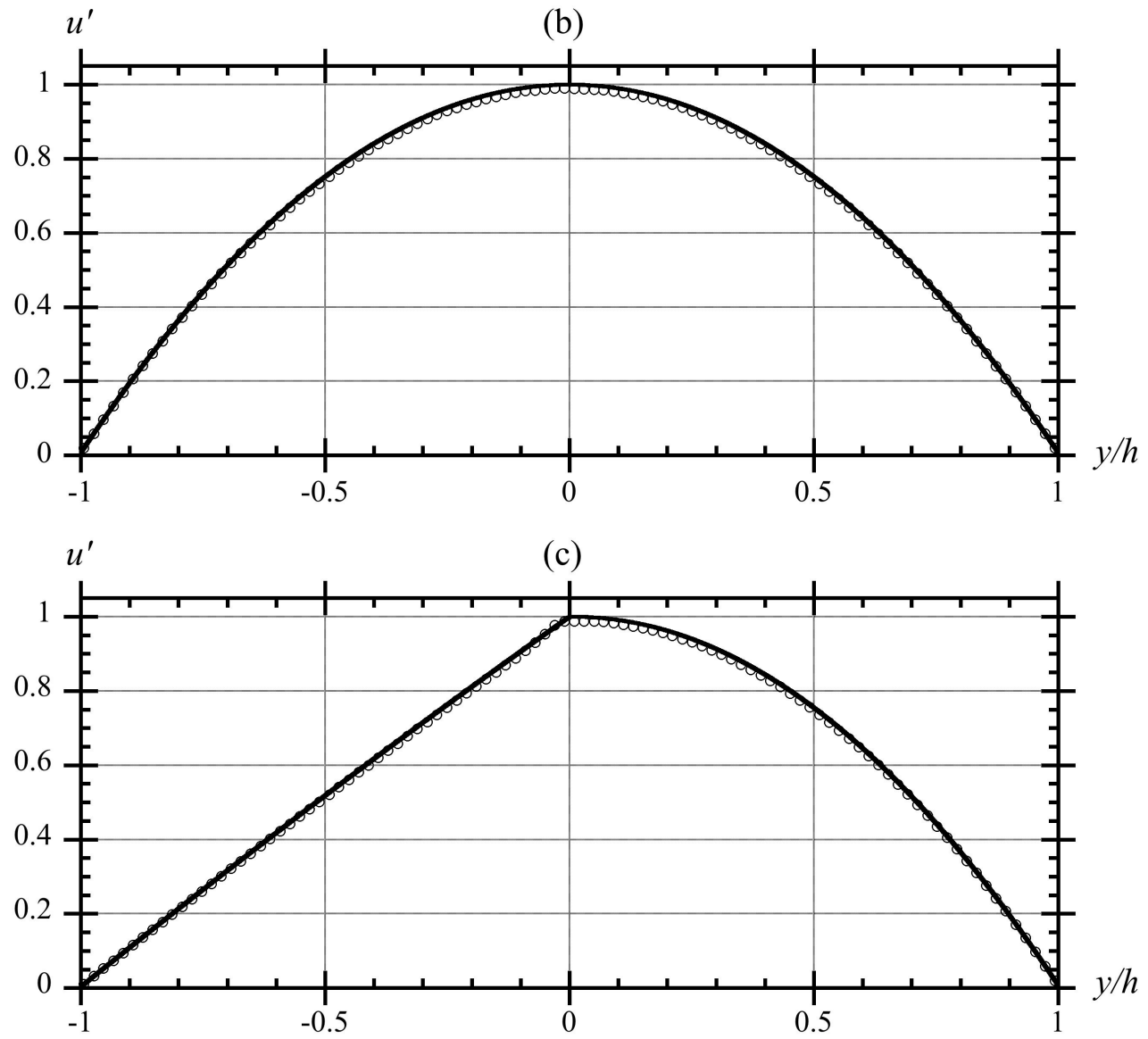

Figure 1.5: Two-fluid Poiseuille flow (Fig. 1.2). Non-dimensional velocity profiles $u^{\prime}=u / u_{1}^{\max }$ for $\mathrm{Re}=1000$ and $\mathrm{Ma}=0.005:(-)$ analytical; (o) LB results for $N=300 \mathrm{LBM}$ nodes (only $33 \%$ of nodes are plotted for clarity). (a) $\rho_{1}^{\prime} / \rho_{2}^{\prime}=1$ and $\nu_{1} / \nu_{2}=0.1$, (b) $\rho_{1}^{\prime} / \rho_{2}^{\prime}=100$ and $\nu_{1} / \nu_{2}=1$, (c) $\rho_{1}^{\prime} / \rho_{2}^{\prime}=1000$ and $\nu_{1} / \nu_{2}=0.0667$. 


\begin{tabular}{c||c|c|c|c|c}
\hline$N_{x}=N_{y}$ & $\sigma_{12}^{\prime}$ & $k^{\prime}$ & $\beta^{\prime}$ & $\Delta p\left(\mathrm{~N} / \mathrm{m}^{2}\right)$ & $L_{2}$-norm $(\%)$ \\
\hline 64 & $4.3410^{-3}$ & 0.28 & 1.6 & 0.389 & 2.7 \\
128 & $2.1710^{-3}$ & 0.14 & 0.8 & 0.395 & 1.23 \\
256 & $1.0910^{-3}$ & 0.07 & 0.4 & 0.397 & 0.72 \\
\hline
\end{tabular}

Table 1.3: Stationary bubble case. Comparison of computed and analytical $(0.4$ $\mathrm{N} / \mathrm{m}^{2}$ ) pressure jumps $\Delta p$ for stationary circular droplets, as a function of LBM discretization.

$\sigma_{12}=410^{-3} \mathrm{~N} / \mathrm{m}$ and $\tau_{g}^{\prime}=1$. Hence, $\Delta t=(\Delta x)^{2} /\left(6 \nu_{1}\right)=5.08610^{-7} \mathrm{~s}, c=153.6$ $\mathrm{m} / \mathrm{s}$, which yields $\sigma_{12}^{\prime}=2.1710^{-3}, \beta^{\prime}=0.8$ and $k^{\prime}=0.14$. Fig. 1.6 shows the time-evolution of the droplet geometry, which as expected gradually relaxes to a circle to both minimize and equalize the interface pressure jump, based on Laplace law. The relaxation time $\tau_{g}^{\prime}=1$ corresponds to a highly viscous fluid, so that the initial droplet relaxes to the equilibrium shape without significant oscillations.

In an accurate two-phase flow model, there should only be negligible flow velocities computed once the bubble reaches its equilibrium shape. In the LBM model, such spurious velocities are eliminated by the correction of the velocity field resulting from the solution of the Poisson equation for the modified pressure in Eq. (1.19). As a verification, Fig. 1.7 shows velocity vectors computed around the bubble interface as a function of time, once its shape has nearly reached equilibrium. We clearly see that spurious velocities gradually decay over time. When the steady state solution is reached, spurious currents have almost completely vanish.

Fig. 1.8 shows the pressure and density profiles computed across the interface, when reaching steady-state. As expected, the density variation is sharp over the interface and occurs over 4 grid cells. At this stage (Fig. 1.6 (f)), the droplet is nearly circular, with a radius $R^{\prime}=68$ lattice nodes or $R=5.310^{-3} \mathrm{~m}$, for which Laplace law predicts an expected pressure jump $\Delta p=0.75 \mathrm{~N} / \mathrm{m}^{2}\left(\Delta p^{\prime}=\right.$ $3.17910^{-5}$ ), whereas on Fig. 1.8 we see a computed average pressure jump of about $0.745 \mathrm{~N} / \mathrm{m}^{2}$ (relative difference $0.67 \%$ ). 


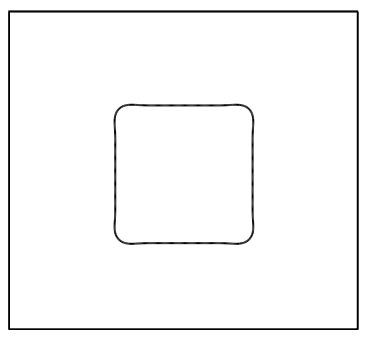

(a) $t^{\prime}=0$

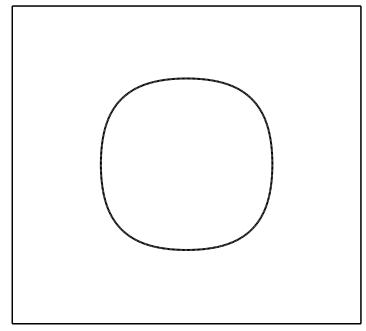

(d) $t^{\prime}=8000$

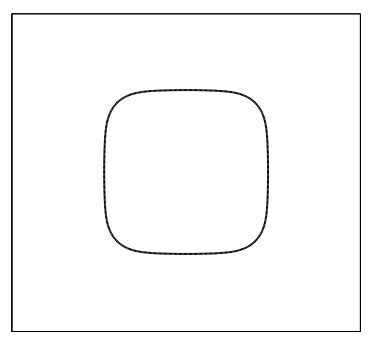

(b) $t^{\prime}=3000$

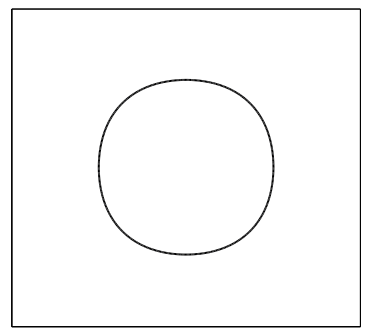

(e) $t^{\prime}=12000$

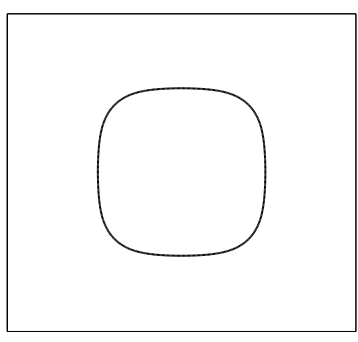

(c) $t^{\prime}=5000$

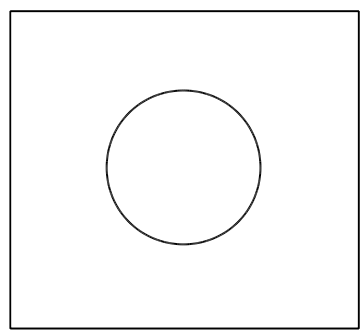

(f) $t^{\prime}=25000$

Figure 1.6: Time evolution of the shape of an initially rectangular droplet towards a circle of radius $R^{\prime}=68\left(\rho_{1}^{\prime}=600, \rho_{1} / \rho_{2}=100\right)$, as a result of Laplace law; $t^{\prime}$ is non-dimensional time of computations (i.e., number of time steps).

\subsubsection{Rising bubble in quiescent fluid}

The dynamic behavior of a lighter fluid $\left(\rho_{2}, \nu_{2}\right)$ bubble rising in a heavier fluid $\left(\rho_{1}, \nu_{1}\right)$ under the buoyancy (gravitational) force is a standard test case extensively used for validating two-phase flow simulations. Although the LBM simulation setup in terms of grid initialization and boundary conditions is straightforward, the flow structure computed around the bubble is quite complex and governed by competing effects of viscosity, buoyancy, and surface tension forces. Several experimental studies have been conducted to measure the rise and deformation of single bubbles in a quiescent fluid [31, 32, which indicate that the bubble shape greatly varies according to various flow regimes defined by values of non-dimensional parameters, such as the Bond number Bo (also known as Eotvos number, the ratio of gravity to surface tension forces), the Reynolds number Re, and the Morton 


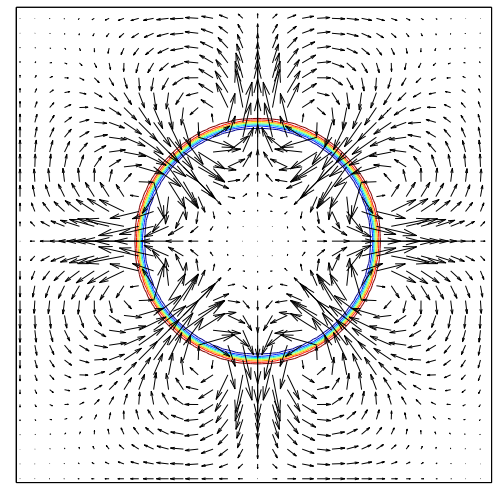

(a) $t^{\prime}=25000$

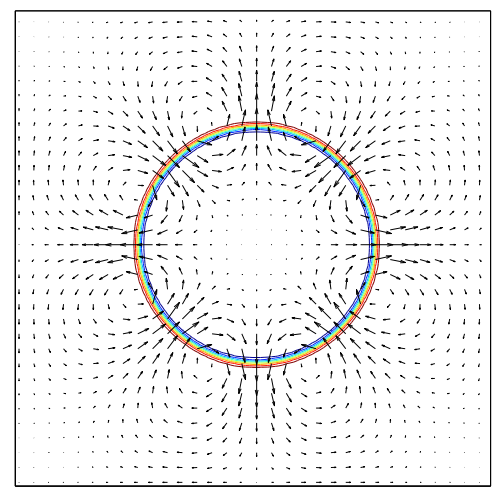

(c) $t^{\prime}=40000$

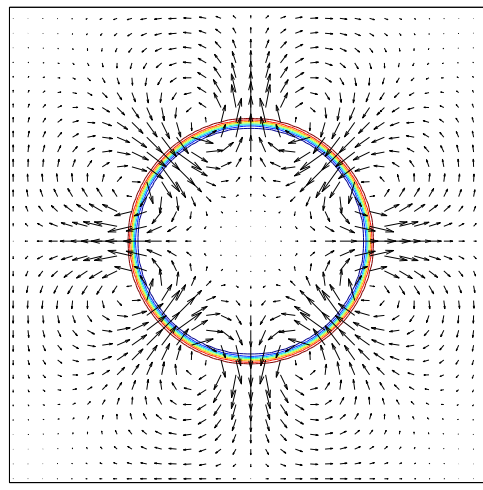

(b) $t^{\prime}=30000$

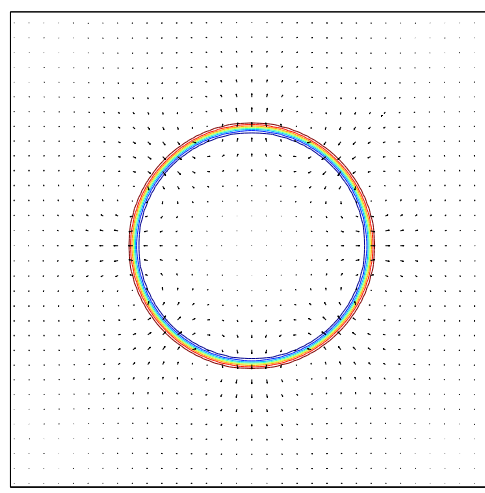

(d) $t^{\prime}=50000$

Figure 1.7: Velocity vectors computed around the bubble interface at four different times equal or larger than the largest time in Fig. 1.6.

number Mo, defined as,

$$
\text { Bo }=\frac{g D^{2}}{\sigma_{12}}\left(\rho_{1}-\rho_{2}\right) \quad ; \quad \operatorname{Mo}=\frac{g \mu_{1}^{4}}{\sigma_{12}^{3} \rho_{1}}\left(1-\frac{\rho_{2}}{\rho_{1}}\right) \quad ; \quad \operatorname{Re}=\frac{U D}{\nu_{1}}
$$

where $U$ is the bubble terminal velocity and $D$ its (equivalent) diameter, $g$ is the gravitational acceleration, and $\nu_{1}$ and $\mu_{1}$ are the kinematic and dynamic viscosities of the heavier fluid, respectively. The terminal shapes of individual rising bubbles were experimentally observed for a range of Reynolds and Bond numbers [31], and can be generally regrouped into the following cap shape regimes: (a) spherical, (b) 
ellipsoidal, (c) curved ellipsoidal. In the spherical regime, for small Bo, surface tension is dominant. The large surface tension force prevents the deformation of the bubble under inertia and viscous forces; consequently, the shape of the bubble remains (nearly) spherical during its rise. When increasing the Reynolds and Bond numbers, the contribution of surface tension gradually becomes less important as compared to inertia, and the terminal shape of the bubble becomes ellipsoidal for moderate Reynolds and Bond numbers $(10<\operatorname{Re}<500$ and $10<$ Bo $<100)$, and spherical for high Reynolds and Bond numbers.

\begin{tabular}{c||c|c|c|c|c|c}
\hline Case & $\nu_{1}^{\prime}$ & $k^{\prime}$ & $\beta^{\prime}$ & $g^{\prime}$ & Bo & Mo \\
\hline (a) & $2.010^{-3}$ & 0.266 & 1.481 & $1.85310^{-11}$ & 0.1 & 0.001 \\
(b) & $2.010^{-3}$ & 0.266 & 1.481 & $1.85310^{-9}$ & 10 & 0.1 \\
(c) & $1.010^{-2}$ & 0.210 & 1.171 & $1.46510^{-8}$ & 100 & 1000 \\
\hline
\end{tabular}

Table 1.4: LBM parameters for rising bubble computations.

In the LBM simulations, a circular fluid bubble of density $\rho_{2}^{\prime}=6$ and initial diameter $D_{0}^{\prime}=60$ is located one bubble diameter above the bottom of a rectangular domain discretized with $256 \times 1024$ LBM cells, filled with a fluid of density $\rho_{1}^{\prime}=$ 6000 (hence $\rho_{1}^{\prime} / \rho_{2}^{\prime}=1000$ ); the fluid viscosity ratio is $\mu_{1}^{\prime} / \mu_{2}^{\prime}=1000$. Both fluids are assumed to be stationary at initial time $t^{\prime}=0$ and we specify a periodic boundary condition on the lateral sides of the domain and a bounce-back condition on the top and bottom boundaries. Simulations are run for 3 test cases (a to c) with different Mo and Bo values, given in Table 1.4 together with LBM and other flow parameters, corresponding to the three flow and bubble shape regimes discussed above.

Since we only solve for a two-dimensional (2D) flow, we cannot compare our LBM simulation results to experiments. However, we can validate results by comparing them to an independent 2D numerical solution, such as that of Sun et al. [33], who used a hybrid volume-of-fluid and level set (VOSET) method to simulate 
incompressible two-phase flows. In Fig. 1.9, the terminal shapes of the bubbles and the velocity fields computed with the LBM for the 3 cases are compared to Sun et al.'s results. We see that both the predicted bubble shape and flow fields agree well with the reference solution.

Fig. 1.10 further shows the computed time evolution of the bubble shape during its rise, for the case of Fig. 1.9 (c). During the early stages of the simulation, buoyancy forces are dominant and the bubble starts rising; as it picks up speed, viscous drag gradually changes the bubble shape, bending it downstream. Eventually, the terminal shape of the bubble is formed when buoyancy, surface tension, and viscous forces are balanced.

\subsubsection{Rayleigh-Taylor instability}

The classical Rayleigh-Taylor instability at the interface between two immiscible fluids, with the heavier fluid being located above the lighter fluid, is used to demonstrate the accuracy of our LBM model to solve more complex two-phase flows, in which the interface becomes extremely deformed. Here, the heavier fluid will gradually sink into the lighter fluid, which is displaced upwards, under the influence of gravity. The dimensionless numbers that are important in this test case are the Atwood number and the Reynolds number, which are defined as,

$$
A=\frac{\rho_{1}-\rho_{2}}{\rho_{1}+\rho_{2}} \quad \text { and } \quad \operatorname{Re}=\frac{\sqrt{L g} L}{\nu},
$$

where $L$ is the width of the channel, and $\rho_{1}$ and $\rho_{2}$ are the densities of the heavy and light fluids, respectively. We set-up this simulation following [8] and specify no-slip boundary conditions along the top and bottom boundaries, and periodic boundary conditions on the lateral boundaries; gravity is chosen to satisfy $\sqrt{L^{\prime} g^{\prime}}=0.04$. The kinematic viscosity is the same for both fluids $\nu=\nu_{1}=\nu_{2}$, and the Atwood and Reynolds numbers are 0.5 and 256, respectively, with accordingly $\rho_{1} / \rho_{2}=3$, $\rho_{2}^{\prime}=6, k^{\prime}=0.07$ and $\beta^{\prime}=0.4$ LBM simulations are carried out in a grid 
with $256 \times 1024$ lattice nodes. Fig. 1.11 compares results of our method to the independent 2D numerical results of [8], for four selected time steps; the agreement between both methods is quite good.

\subsubsection{Breaking wave}

Previous work [34, 35] shows that a periodic sinusoidal wave of large amplitude, with the initial velocities being calculated from linear wave theory, is not stable and rapidly breaks, since the initial velocity field is not in equilibrium with the initial wave profile, for the fully nonlinear flow equations. To limit computational time, as in this earlier work, the simulation is assumed to be $2 \mathrm{D}$ and periodic in the flow direction. This characteristic makes such periodic sinusoidal waves a convenient and efficient way of studying wave breaking [1].

According to linear wave theory, in axes $(x, z)$, the initial wave velocity and interface shape are given by ,

$$
\begin{aligned}
& \eta=\frac{H}{2} \cos (k x) \\
& u=\frac{H}{2} \omega \frac{\cosh k(h+z)}{\sinh (k h)} \cos (k x) \\
& v=\frac{H}{2} \omega \frac{\sinh k(h+z)}{\sinh (k h)} \sin (k x)
\end{aligned}
$$

where $\omega$ is the wave angular frequency, $k$ the wavenumber, and other wave parameters are defined in Fig. 1.12 .

Fig. 1.13 shows the time evolution computed with the LBM, of a high amplitude sinusoidal wave with $H / L=0.13$ in depth $h / L=0.25$. The number of grid nodes used in this simulation is $512 \times 256$. We see that the wave is not stable and rapidly overturns and breaks, after traveling for about one wavelength from initialization. Results obtained for the plunging breaker shape are qualitatively similar to those of earlier numerical solutions [34, 35]. 


\subsection{Conclusions and outlook}

In this paper, we reported on the development, efficient GPGPU implementation, and numerical validation, of an LBM model for the simulation of two-phase flows of fluids with large density ratios (up to at least $\rho_{1} / \rho_{2}=1000$ ), and possibly large viscosity ratios as well. In the model, we introduced three sets of LBM particle distribution functions to solve: (i) the "pressureless" Navier-Stokes equations in both fluids; (ii) the convection-diffusion Cahn-Hilliard equation for the two-fluid interface motion (including surface tension effects); and (iii) a (pressure) Poisson equation for correcting the pressureless velocity field. As a result of this homogeneous LBM formulation, this 2D scheme could be efficiently implemented in a GPGPU framework, owing to both the locality and simplicity of LB operators. This LBM scheme was applied to the simulation of various analytical or numerical benchmark problems, showing both good convergence towards reference results and efficiency in terms of computational time. This good agreement confirmed the prediction of the theoretical Chapman-Enskog expansions, of convergence of the scheme to the above-mentioned macroscopic equations.

While validating the LBM scheme for several benchmark problems, we found a linear convergence rate of the $L_{2}$-norm of numerical errors to the reference solution. This is consistent with the truncation errors of both the Chapman-Enskog expansions and time updating schemes corresponding to the various particle distribution functions. Additionally, some of the numerical errors result from the computation of the $-\mu\left(\partial_{\alpha} u_{\beta}+\partial_{\beta} u_{\alpha}\right) \partial_{\beta}(1 / \rho)$ terms in Eq. 1.33 , which are added to the standard LBM scheme (Eq. (1.21) ) as an equivalent body force, to simulate the complete Navier-Stokes equations. Since these extra forcing terms are highly varying in both space and time near the two-fluid interface, unlike standard body

forces such as gravity, additional terms appear in the Chapman-Enskog expansion 
for the momentum equation, that only linearly vanish with grid size. To obtain more accurate (and faster converging) numerical results, these terms should not be neglected, especially for high fluid density and viscosity ratios. Hence, higherorder LBM schemes for the body force terms should be used (e.g [36]). We are currently addressing this issue and developing such second-order schemes, which will be reported on in a future paper.

Note, a simple temporary solution to decrease this truncation error would have been to increase the interface thickness $W$, thus yielding smaller density gradients across the interface and hence a smaller total body force. While the nature of the real fluid interface is to be diffusive, however, this occurs over a very small length scale, that possibly is below LBM grid resolution, so that the interface thickness should be kept small. Additionally, the analytical reference solutions, such as for the two phase Poiseuille flow, are derived assuming a sharp phase interface. Hence, in order to compare our LBM results to theoretical solutions, we decided to limit the interface thickness to only 4 LBM grid cells.

A second significant model improvement that will be the object of future work, is the derivation of multiple relaxation time (MRT) collision operators. In these, the particle distribution functions are transformed to a well-defined moment space before doing the relaxation step. Several different relaxation rates can thus be used for the collision steps, unlike the single relaxation time used in the currently implemented SRT approach. This typically leads to a more efficient and stable numerical scheme, particularly for low fluid viscosities and high Reynolds numbers.

Finally, once the model has been further improved, such as by using the MRT, the GPGPU implementation will be extended to solving three-dimensional (3D) flows. As the iterations for solving the pressure Poisson equations may become much more computationally demanding in 3D, we are planning to explore using a 
multigrid algorithm to speed up this part of the simulations.

\section{Acknowledgements}

The authors gratefully acknowledge support for this research from the nVIDIA Academic Partnership Program (APP) as well as Grant OCE-09-27014 from the US National Science Foundation. In addition, MK acknowledges support from the Deutsche Forschungsgemeinschaft by funding RTG MUSIS.

\section{List of References}

[1] P. Lubin, S. Vincent, S. Abadie, J.-P. Caltagirone, Three-dimensional large eddy simulation of air entrainment under plunging breaking waves, Coastal Engineering 53 (2006) 631-655.

[2] C. Janßen, S.T. Grilli and M. Krafczyk. On enhanced non-linear free surface flow simulations with a hybrid LBM-VOF approach. Computers and Mathematics with Applications, 65 (2) (2013) 211-229.

[3] D. Jacqmin, Calculation of two-phase Navier-Stokes flows using phase-field modeling, Journal of Computational Physics 155 (1999) 96-127.

[4] S. O. Unverdi, G. Tryggvason, A front-tracking method for viscous, incompressible, multi-fluid flows, Journal of Computational Physics 100 (1992) 2537.

[5] J. Brackbill, D. B. Kothe, C. Zemach, A continuum method for modeling surface tension, Journal of Computational Physics 100 (1992) 335-354.

[6] J. Cahn, J. Hilliard, Free energy of a nonuniform system, Journal of Chemical Physics 28 (1958) 258-267.

[7] M. Swift, W. Osborn, J. Yeomans, Lattice Boltzmann simulation of non-ideal fluids, Physical Review Letters 75 (1995) 830-833.

[8] X. He, S. Chen, R. Zhang, A lattice Boltzmann scheme for incompressible multiphase flow and its application in simulation of Rayleigh-Taylor instability, Journal of Computational Physics 152 (1999) 642-663.

[9] T. Lee, C. Lin, A stable discretization of the lattice Boltzmann equation for simulation of incompressible two-phase flows at high density ratio, Journal of Computational Physics 206 (2005) 16-47. 
[10] T. Inamuro, T. Ogata, S. Tajima, N. Konishi, A lattice Boltzmann method for incompressible two-phase flows with large density differences, Journal of Computational Physics 198 (2004) 628-644.

[11] S. Chapman, T. Cowling, The mathematical theory of nonuniform gases, Cambridge University Press, 1970.

[12] J. Tölke, M. Krafczyk, TeraFLOP computing on a desktop PC with GPUs for 3D CFD, International Journal of Computational Fluid Dynamics 22 (2008) 443-456.

[13] D. Rothman, J. Keller, Immiscible cellular-automaton fluids, Journal of Statistical Physics 52 (1988) 1119-1124.

[14] X. Shan, H. Chen, Lattice Boltzmann model for simulating flows with multiple phases and components, Physical Review E47 (1993) 1815-1819.

[15] T. Inamuro, T. Ogata, F. Ogino, Numerical simulation of bubble flows by the lattice Boltzmann method, Future Generation Computer Systems 20 (2004) 959-964.

[16] J. Tölke, M. Krafczyk, Implementation of a lattice Boltzmann kernel using the compute unified device architecture developed by nVIDIA, Computing and Visualization in Science 1 (2008) 29-39.

[17] C. Janßen, M. Krafczyk, Free surface flow simulations on GPGPUs using LBM, Computers and Mathematics with Applications 61 (12) (2011) 35493563.

[18] Schonherr, M., Kucher, K., Geier, M., Stiebler, M., Freudiger, S., Krafczyk, M., Multi-thread implementations of the lattice Boltzmann method on nonuniform grids for CPUs and GPUs, Computers and Mathematics with Applications, Vol. 61, 12, 3730-3743 (2011).

[19] Y. Qian, d'Humieres, Lallemand, Lattice BGK models for Navier Stokes equation, Europhysical Letters 17 (1992) 479-84.

[20] D. Yu, R. Mei, L. Luo, W. Shyy, Viscous flow computations with the method of lattice Boltzmann equation, Progress in Aerospace Sciences 39 (2003) 329 367.

[21] J. Buick, C. Greated, Gravity in lattice Boltzmann models, Physical Review E61 (2000) 5307-5320.

[22] X. He, L. S. Luo, Theory of the lattice Boltzmann method: From the Boltzmann equation to the lattice Boltzmann equation, Physical Review E56 (1997) 6811-6817. 
[23] M. Swift, W. Osborn, J. Yeomans, Lattice Boltzmann simulation of liquid-gas and binary fluid systems, Physical Review E54 (1996) 5041-5052.

[24] D. Grunan, Lattice Methods for Modeling Hydrodynamics, PhD thesis Colorado State University, 1993.

[25] nVIDIA, http://www.nvidia.com/object/cuda_develop.html, NVIDIA CUDA Programming Guide (2010).

[26] nVIDIA, http://www.nvidia.com/cuda, NVIDIA CUDA (2011).

[27] T.Lee, Effects of incompressibility on the elimination of parasitic currents in lattice Boltzmann method for binary fluids, Computer and mathematics 58 (2009) 987-994.

[28] M. J. South, A. P. Hooper, Linear growth in two fluid plane Poiseuille flow, Journal of Fluid Mechanics 381 (1999) 121-139.

[29] M. Gross, N. Moradi, G. Zikos, F. Varnik, Shear stress in nonideal fluid lattice Boltzmann simulations, Physical Review E83 (2011) 017701.

[30] S. Freudiger, Entwicklung eines parallelen, adaptiven, komponentenbasierten Stroemungskerns fuer hierarchische Gitter auf Basis des Lattice-BoltzmannVerfahrens, PhD thesis, Technischen Universitaet Carolo-Wilhelmina zu Braunschweig, 2009.

[31] R. Clift, J. Grace, M. Weber, Bubbles Drops and Particles, Academic Press, 1978.

[32] D. Bhaga, M. Weber, Bubbles in viscous liquids: shapes, wakes and velocities, Journal of Fluid Mechanics 105 (1981) 61-85.

[33] D. Sun, W. Tao, A coupled volume-of-fluid and level set (VOSET) method for computing incompressible two-phase flows, International Journal of Heat and Mass Transfer 53 (2010) 645-655.

[34] M. S. Longuet-Higgins, E. D. Cokelet, The deformation of steep surface waves on water. I. a numerical method of computation, Proceedings of the Royal Society of London A350 (1976) 1-26.

[35] S. T. Grilli, J. Skourup, I. A. Svendsen, An efficient boundary element method for nonlinear water waves, Engineering Analysis with Boundary Elements 6 (1989) 97-107.

[36] Z. Guo, C. Zhen, B. Shi, Discrete lattice effects on the forcing term in the lattice Boltzmann method, Physical Review A65 (2002) 042102.

[37] J. Huang, Lattice Boltzmann Study of Near-Wall Multi-Phase and MultiComponent Flows, PhD thesis national university of Singapore, 2009. 
[38] X. He, L.-S. Luo, Lattice Boltzmann model for the incompressible navierstokes equation, Journal of Statistical Physics 88 (1997) 927-944.

\section{List of References}




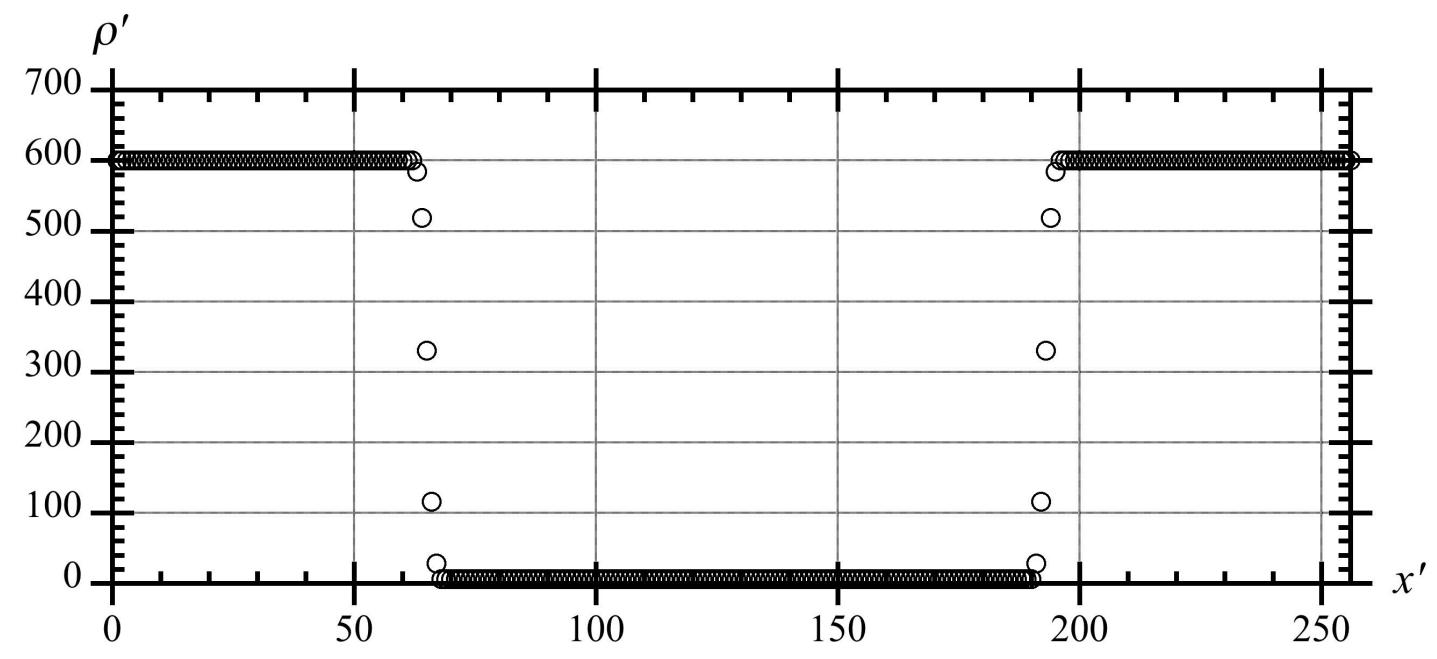

$p\left(\mathrm{~N} / \mathrm{m}^{2}\right)$

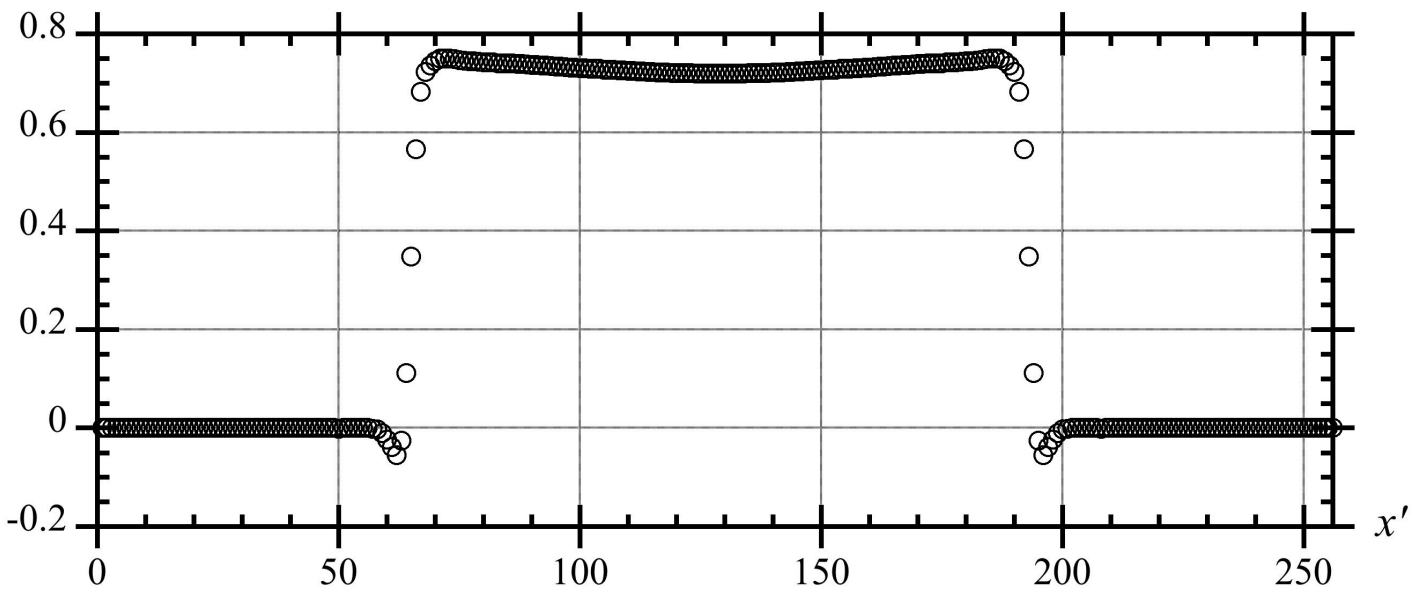

Figure 1.8: Case of Fig. 1.6 (f) (steady-state). (o) LBM simulation of nondimensional density and pressure profiles across the domain mid-axis. 

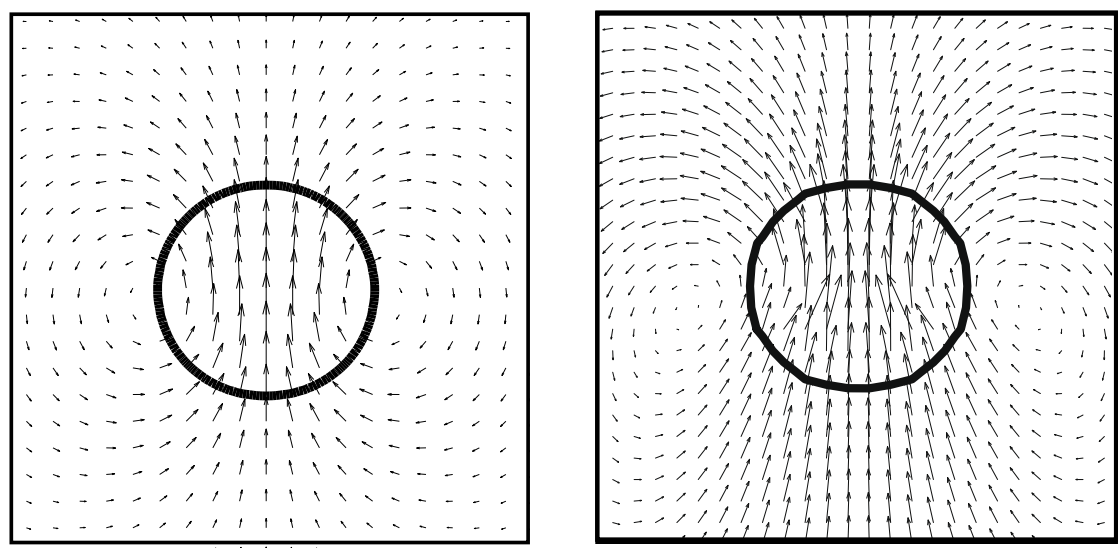

(a) $\mathrm{Bo}=0.1, \mathrm{Mo}=0.001$
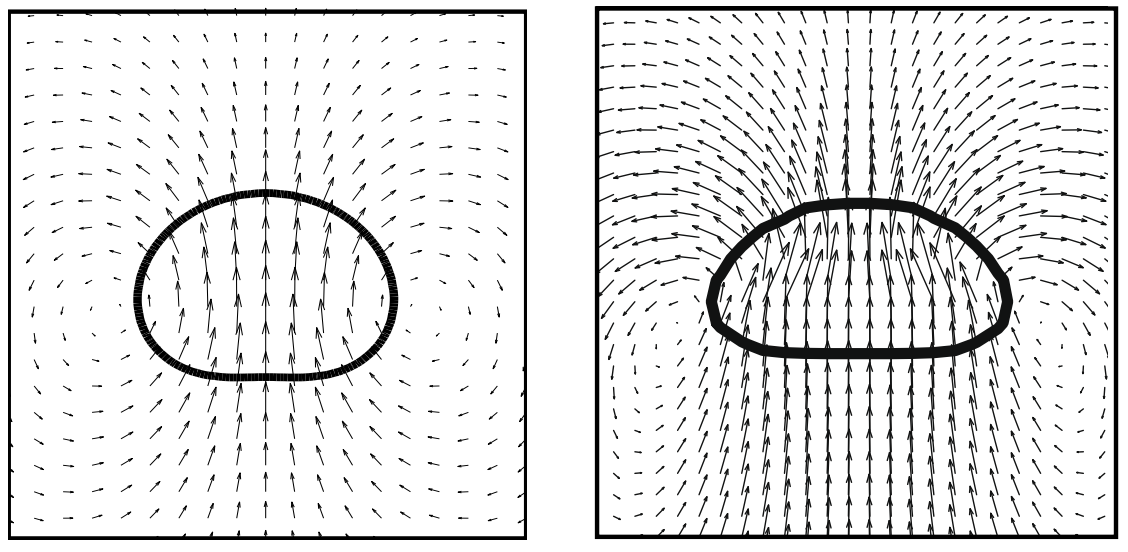

(b) $\mathrm{Bo}=10, \mathrm{Mo}=0.1$
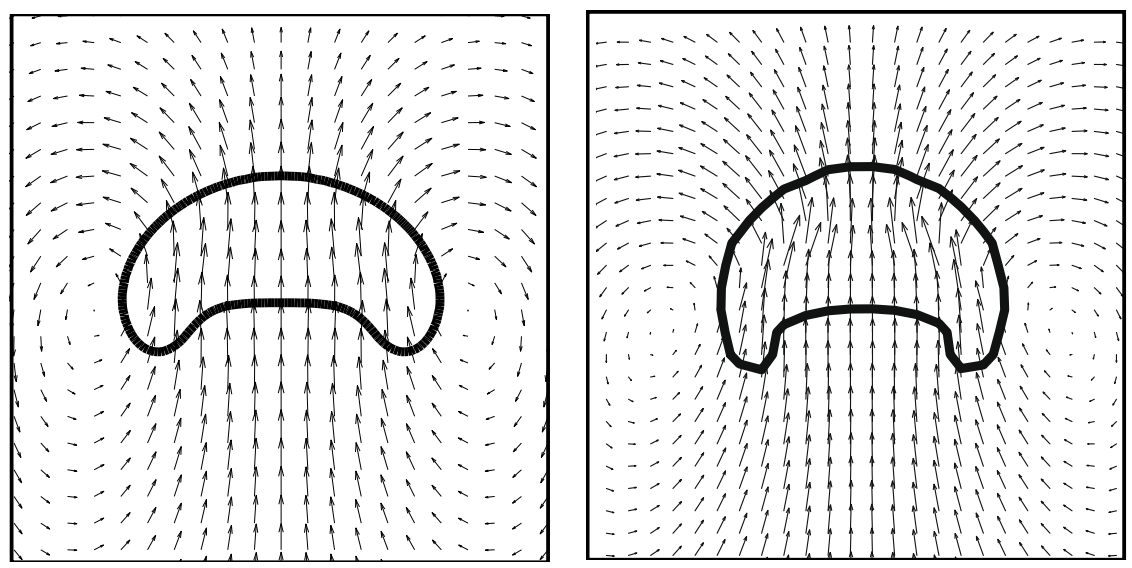

(c) $\mathrm{Bo}=100, \mathrm{Mo}=1000$

Figure 1.9: Terminal shape and flow velocity vectors for a bubble of density $\rho_{2}^{\prime}=6$ rising under buoyancy in a fluid with density $\rho_{1}^{\prime}=6000, \mu_{1}^{\prime} / \mu_{2}^{\prime}=1000$ : (rightward panels) VOSET results of [33]; (leftward panels) present LB results (see Table 1.4). 


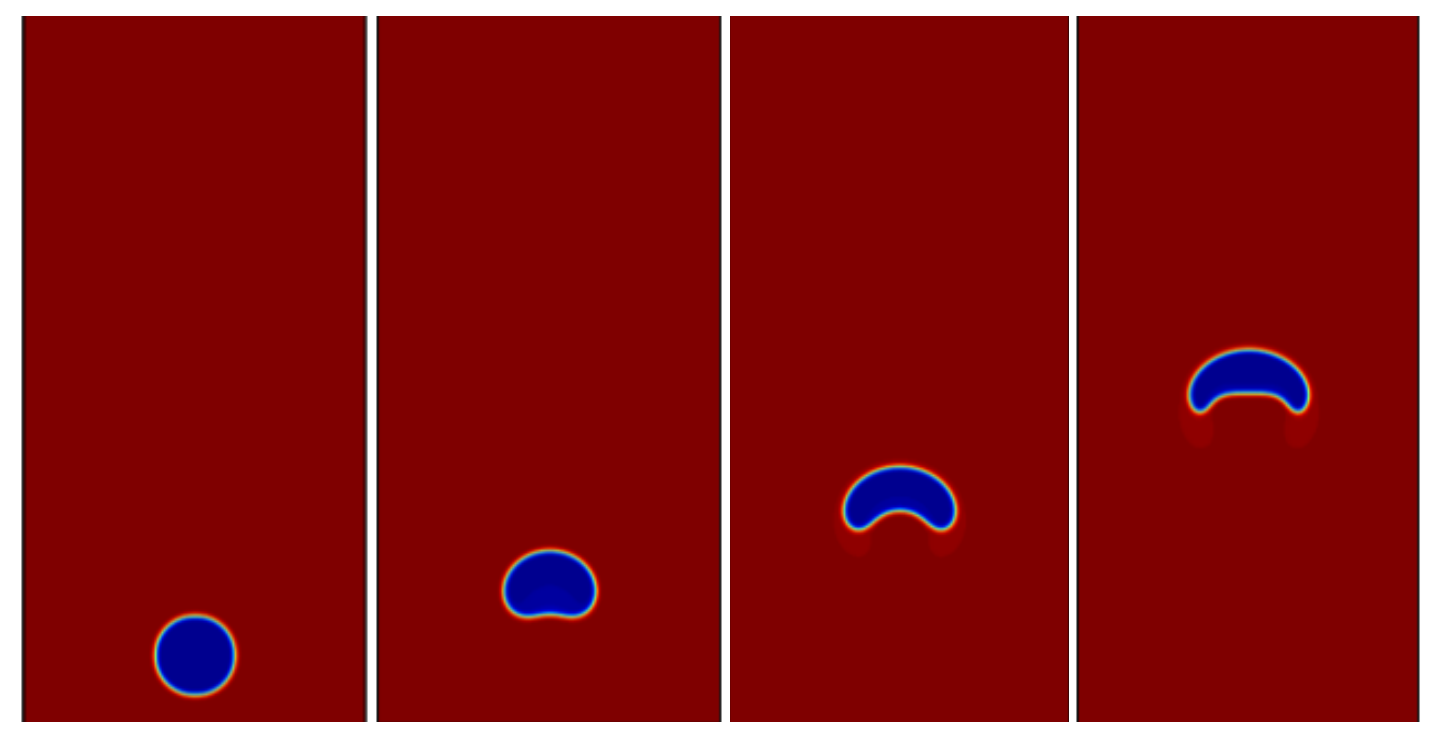

Figure 1.10: Time evolution of a rising bubble shape for $\rho_{1}^{\prime} / \rho_{2}^{\prime}=1000$, Bo $=100$, $\mathrm{Mo}=1000$ (case of Fig. 1.9 (c)). From left to right, for $t^{\prime}=0,10^{3}, 2.10^{3}, 4.10^{3}$. Note, only half the domain height is plotted. 


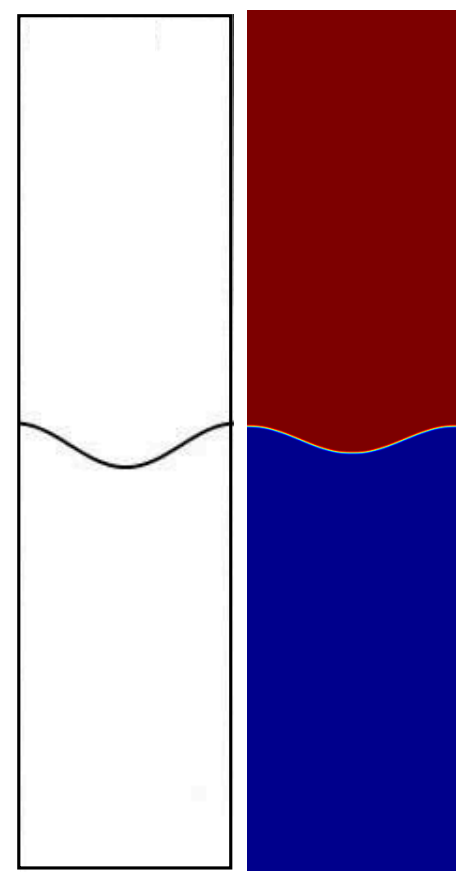

(a) $t^{\prime}=0.0$

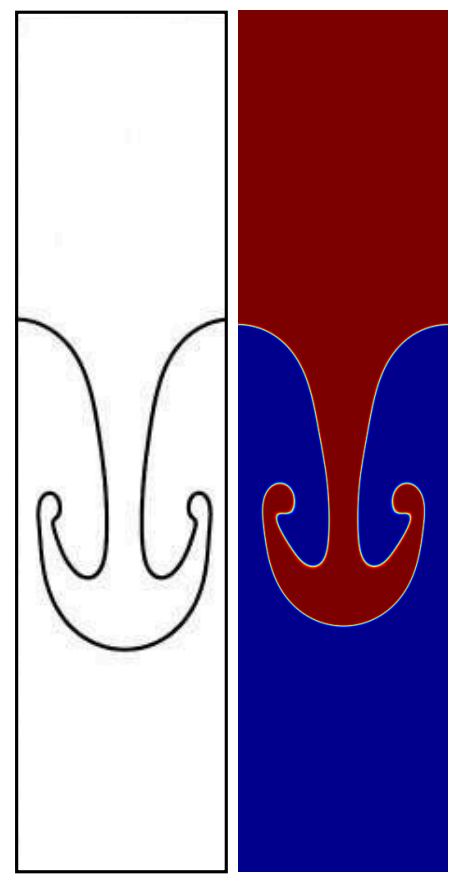

(c) $t^{\prime}=3.0$

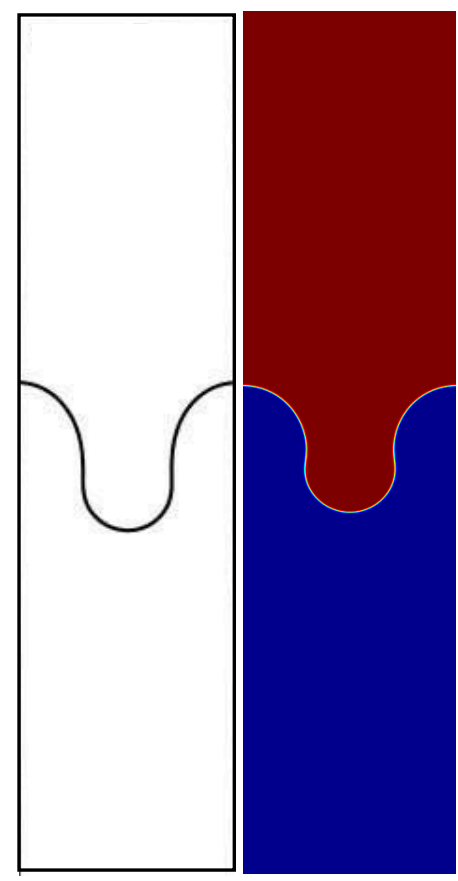

(b) $t^{\prime}=1.5$

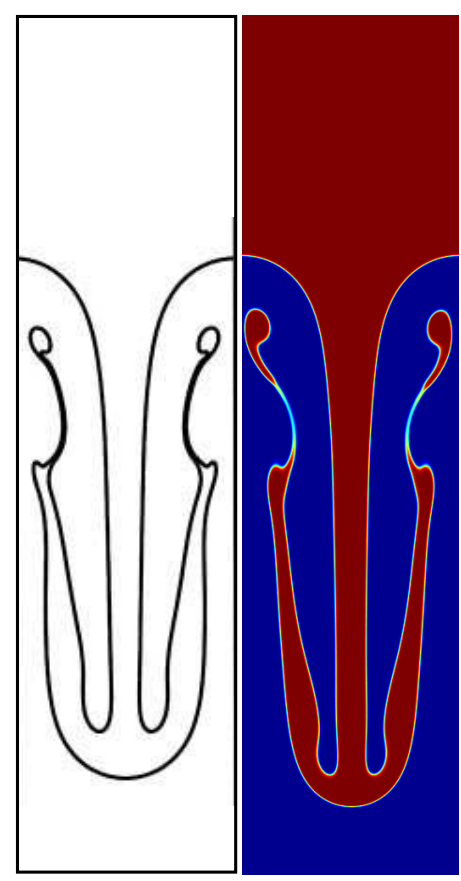

(d) $t^{\prime}=4.5$

Figure 1.11: Rayleigh-Taylor instability problem for $\rho_{1} / \rho_{2}=3, A=0.5, \operatorname{Re}=256$. Time evolution of the two-fluid interface for four dimensionless times $t^{\prime}=t / \sqrt{L / g}$ : (leftward panels) results of [8]; (rightward panels) present LB results. 


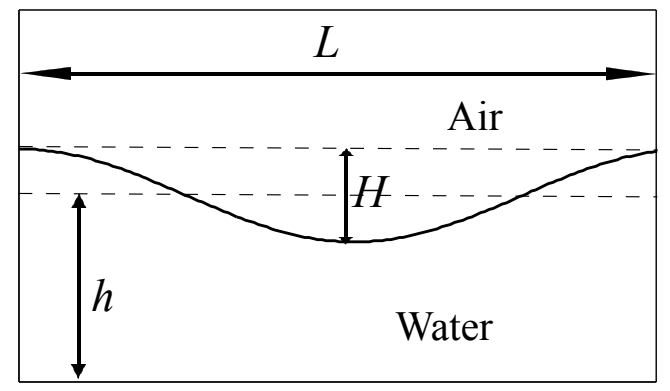

Figure 1.12: Definition sketch for initial interface profile of a large amplitude sinusoidal wave. 


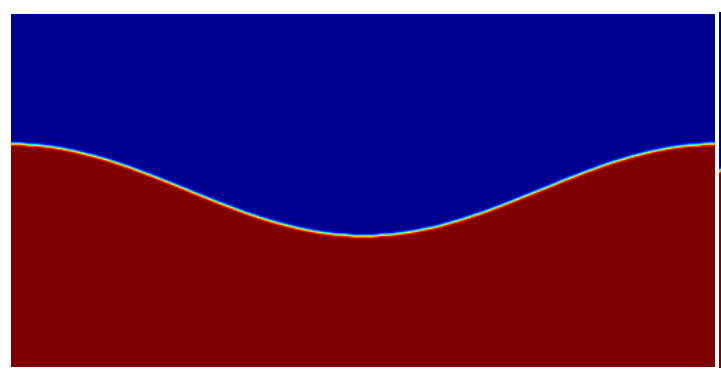

(a) $t^{\prime}=0.0$

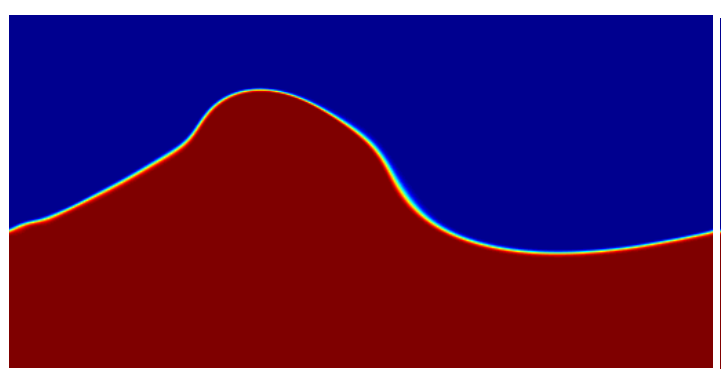

(c) $t^{\prime}=1.33$

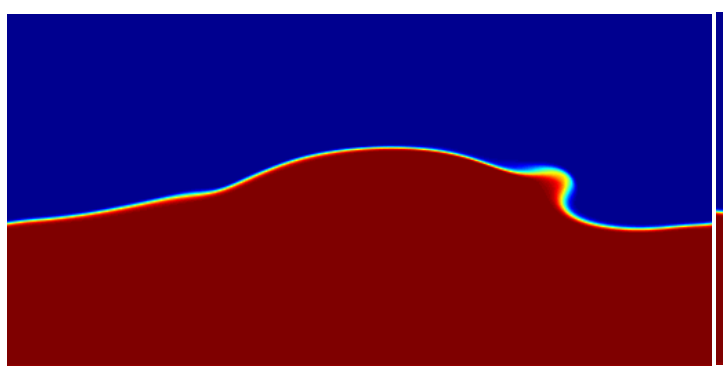

(e) $t^{\prime}=2.0$

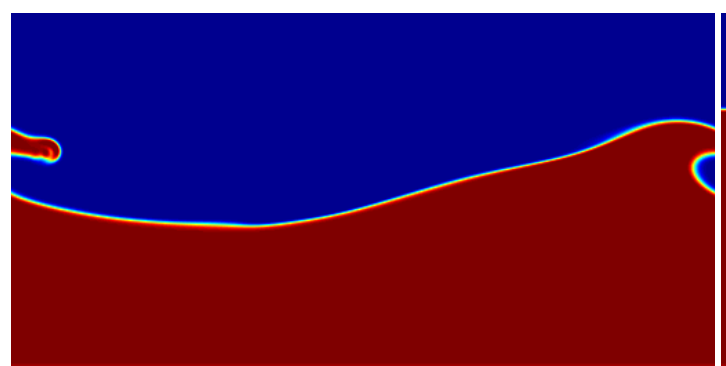

(g) $t^{\prime}=2.66$

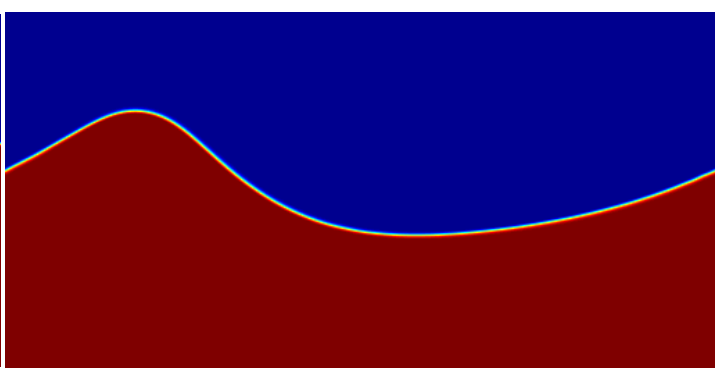

(b) $t^{\prime}=0.33$

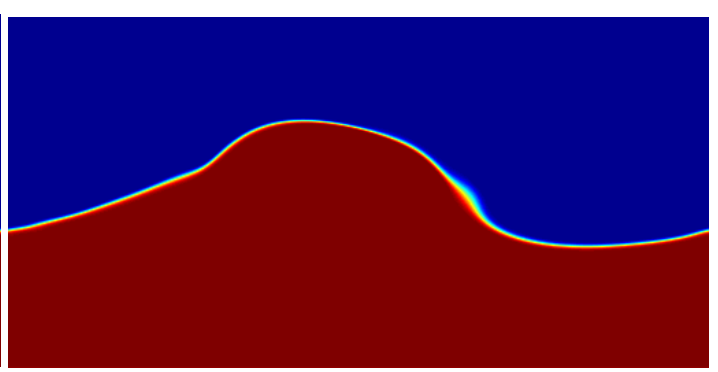

(d) $t^{\prime}=1.66$

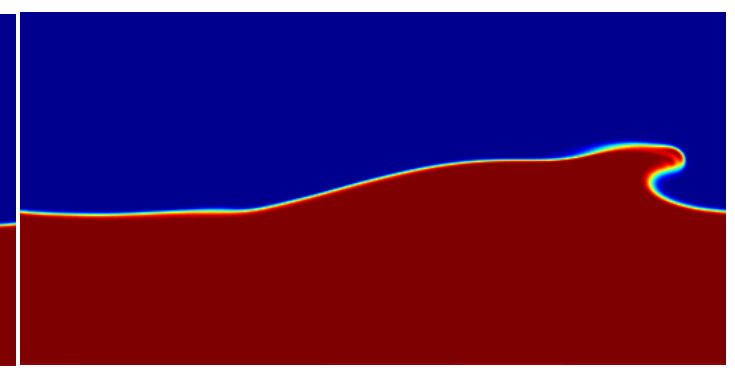

(f) $t^{\prime}=2.33$

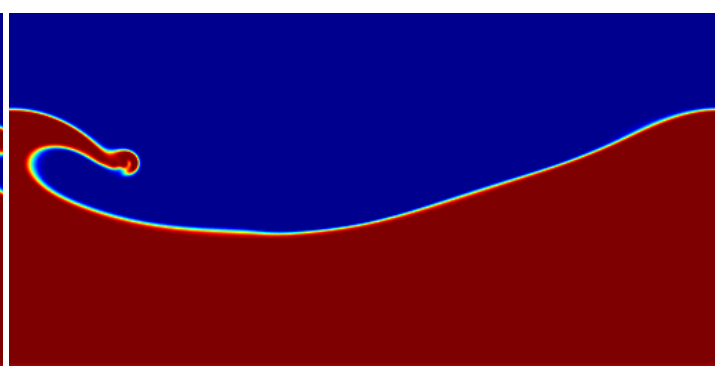

(h) $t^{\prime}=3.0$

Figure 1.13: Space-periodic wave breaking (Fig. 1.12). Time evolution of an overturning breaking wave with $H / L=0.13$ and $h / L=0.25$ (dimensionless time $\left.t^{\prime}=t / \sqrt{L / g}\right)$. 


\title{
CHAPTER 2
}

\section{An efficient lattice Boltzmann multiphase model for 3D flows with large density ratios at high Reynolds numbers}

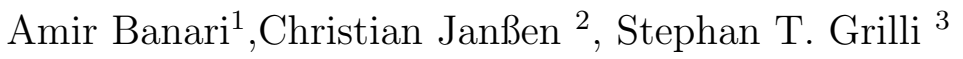 \\ Submitted to Computers and Mathematics with Applications
}

\footnotetext{
${ }^{1}$ Ocean Engineering department, University of Rhode Island, E-mail: amir_banari@my.uri.edu

${ }^{2}$ Institute for Fluid Dynamics and Ship Theory, Hamburg University of Technology, Germany, E-mail: mail@christian-janssen.de

${ }^{3}$ Ocean Engineering department, University of Rhode Island, E-mail: grilli@oce.uri.edu
} 


\begin{abstract}
We report on the development, implementation and validation of a new Lattice Boltzmann method (LBM) for the numerical simulation of three-dimensional multiphase flows (here with only two components) with both high density ratio and high Reynolds number. This method is based in part on, but aims at achieving a higher computational efficiency than Inamuro et al.'s model [T. Inamuro, T. Ogata, S. Tajima and N. Konishi, A lattice Boltzmann method for incompressible two-phase flows with large density differences, J. Comp. Phys., 198 (2004) 628-644.]. Here, we use a LBM to solve both a pressureless Navier-Stokes equation, in which the implementation of viscous terms is improved, and a pressure Poisson equation (using different distribution functions and a D3Q19 lattice scheme); additionally, we propose a new diffusive interface capturing method, based on the Cahn-Hilliard equation, which is also solved with a LBM. To achieve maximum efficiency, the entire model is implemented and solved on a heavily parallel GPGPU co-processor. The proposed algorithm is applied to several test cases, such as a splashing droplet, a rising bubble, and a breaking ocean wave. In all cases, numerical results are found to agree very well with reference data, and/or to converge with the discretization.
\end{abstract}

\title{
keyword
}

Lattice Boltzmann method, multiphase flows, high density ratio, rising bubble, drop impact, breaking wave

\section{$2.1 \quad$ Introduction}

In recent years, the lattice Boltzmann method (LBM) has become an increasingly attractive, fast, and accurate, alternative modeling method to standard continuum mechanics numerical models, for solving a variety of complex single and 
multiple-fluid flow problems [1]. Besides its versatility, this is in part due to the LBM's ability to be efficiently parallelized for implementation on General Purpose Graphical Processor Units (GPGPUs). Specifically, it has been shown in various publications [15, 11, 16] that LBM methods are especially well-suited for a GPGPU implementation, due to the locality of collision and propagation operators and the explicit nature of the method.

The LBM is based on the Boltzmann equation, which governs the dynamics of molecular probability distribution functions from a microscopic point of view. In the standard LBM implementation, the Boltzmann equation is discretized on an Eulerian mesh, a.k.a. the lattice, yielding a numerical method for computing macroscopic distribution functions on the lattice, in which the macroscopic hydrodynamic quantities, such as pressure and velocity, are obtained as low-order moments of these distribution functions [5, 1]. To the limit of small time step and grid spacing, the LBM solution can be shown to converge towards the solution of the governing macroscopic equations of continuum mechanics [10]. Hence, with the proper selection of the LBM collision operator and distribution functions, the LBM solution can be made to converge to that of the Navier-Stokes equations (NS), including in the presence of a free surface (e.g., Janssen et al. [7]). The explicit nature of the method and the linear formulation of advection terms in the LBM collision-propagation equation provide the numerical scheme with several advantages, such as: (i) a relatively easy implementation (as far as uniform grids are concerned); and (ii) the locality of numerical operators, which allows for a more efficient parallel implementation, particularly on GPGPUs, than for more traditional finite volume of finite element algorithms. These characteristics have made the LBM a widely used tool for solving various complex fluid mechanics problems, such as multiphase flows, micro- and nanoscale flows, flows in porous media, and 
other fluid flow types [8, 9, 1 .

Although many studies of multiphase flows using the LBM have been reported [13, 11], most of these had two significant limitations: (1) the maximum density ratio between fluids is typically limited to 5-10, due to the triggering of local instabilities near the fluid-phase interface for larger ratios; (ii) most of the schemes cannot simulate high Reynolds number flows, due to instabilities resulting from the low relaxation times required for high Reynolds numbers (Re). The effects of either one of these problems are sufficient to make simulations unstable, even in the absence of the other problem.

The practical applications that motivated this research deal with air-sea interactions at the ocean surface in high wind conditions, hence with turbulent (i.e., very large Re values around $10^{8}$ ) two-fluid flows with a high density ratio (order 1000). Hence, our main goal has been to develop an efficient LBM model that overcame these two limitations.

Several LBM studies of multiphase flows with a high density ratio have been proposed, however, either for fairly low Reynolds numbers or using an artificial density ratio instead of the actual one [12]. Two promising concepts were proposed by Lee et.al [8] and Inamuro et al. [9]. Lee et.al [8] used an approach similar to that of He et al. [7], in which they transformed the classical single phase discrete Boltzmann equation, from a mass-momentum to a pressure-momentum formulation. This decreased potential instabilities due to large fluid density gradients near the phase interface. Also, they split up the intermolecular forces for a non-ideal gas into hydrodynamic pressure, thermodynamic pressure, and surface tension force contributions. They reported that "parasitic currents" at the phase interface affected the numerical results as a result of an imbalance between thermodynamic pressure and surface tension forces, resulting from truncation errors 
related to curvature computations. They nearly eliminated this problem by using a thermodynamic identity to recast the intermolecular forcing term from a stress to a potential form. Furthermore, to stabilize their numerical scheme for large density ratios, they used different discretization patterns (i.e., central, biased and mixed differences) at different stages of the simulations. With this scheme, they were able to simulate two-phase flows with density ratio up to 1000 . However, they could not achieve high Reynolds numbers, because stability issues related to low relaxation times were not addressed, and in their scheme relaxation time was still a function of the Reynolds number. To eliminate the numerical instabilities resulting from high density ratios, Inamuro et al. [9] removed the density from the advection part of the LBM equilibrium distribution functions. This in effect eliminated the pressure gradient from the corresponding macroscopic momentum equation, which thus became a "pressureless" NS equations. To retrieve the complete momentum equations and satisfy mass conservation, they subsequently corrected the velocity field by solving a Poisson equation for the pressure field. In their method, unlike in classical LBMs, the fluid viscosity is no longer related to the relaxation time and hence results stay more stable at high Reynolds numbers. Finally, in Inamuro et al.'s method, viscous effects are modeled by specifying the viscous stress tensor as a body force in the LBM collision operator. Proceeding this way, however, yields additional non-physical terms in the corresponding momentum equation, which decreases the model accuracy. In earlier work [5], we modified Inamuro et al.'s method to solve two-dimensional (2D) two-phase flows with high density ratio, by removing the non-physical terms from the momentum equation and formulating the phase interface tracking equations in a more rigorous way, based on the CahnHilliard equations [5]. Additionally, we efficiently implemented our model for a massively parallel solution on a GPGPU. In doing so, we solved all the govern- 
ing equations for each fluid, the interface, and the Poisson equation (required for correcting the velocity field) with a LBM scheme, thus achieving an even higher computational efficiency on the GPGPU. Our method, however, only worked for low Reynolds number flows.

In this paper, in light of this earlier work, we develop a new three-dimensional (3D) LBM model, also based on Inamuro et al.'s [9] approach. As before, we introduce new equilibrium distribution functions to both retrieve NS equations and improve the formulation of surface tension and viscous forces. For the interface capturing part, as in [5], we solve the Cahn-Hilliard equation using a LBM scheme with improved equilibrium distribution functions. In this new 3D model, however, we formulate the latter functions to be able to achieve high Reynolds numbers in the applications without suffering from instability problems. The resulting numerical scheme is computationally demanding, as the Poisson equation must be (iteratively) solved for each time step of the solution, in order to obtain the velocity field correction terms. As before, to achieve high computational efficiency, our LBM code is parallelized and implemented on a GPGPU using the nVIDIA CUDA framework. This approach provides access to the latest generation of GPGPU boards, such as the nVIDIA Tesla C2070 that was used in the present work (448 computing cores; 6 GB of memory; and a double precision computing capability).

The paper is organized as follows. We first develop the LBM equations used to solve for multiphase flows with high density ratios and detail their numerical implementation. The method is then validated for a series of applications, by comparing the present numerical results to reference solutions, for the splashing of droplets on a thin fluid layer, for a rising bubble, and for breaking ocean waves. Finally, we draws some conclusions and provide perspectives for future work. 


\subsection{Governing equations and numerical implementation 2.2.1 Lattice Boltzmann Methods}

The numerical simulation of multiphase flow problems typically involves solving the macroscopic governing Navier-Stokes (NS) equations within each fluid region, which directly provides hydrodynamic parameters such as velocity and pressure. Here, as discussed in the introduction, we instead use the lattice Boltzmann method (LBM) to solve a mesoscopic problem, whose solution converges to that of NS equations. Additionally, our proposed LBM approach is able to solve two-fluid flows whose density ratio is nearly 1,000, typically air and water.

For multiphase flows, a separate model must also be implemented for tracking the motion of interfaces between fluids, which can be represented as sharp or diffused. Here, the latter is assumed and interfaces are tracked by the CahnHilliard advection-diffusion (AD) equation. In the latter, a phase order function is used to smoothly vary fluid properties, such as density and viscosity, across the interface (denoted $\phi$ and varying between arbitrary values of $\phi_{1}$ and $\phi_{2}$ set for fluid 1 and 2 , respectively, with here $\phi_{1}>\phi_{2}$; see details in Section 2.2.3.

As in the two-dimensional (2D) model of Banari et al. [5], in our threedimensional (3D) model, both the NS and $\mathrm{AD}$ equations will be solved by two distinct LBM schemes, with separate sets of distributions functions and collision operators. In both cases, the D3Q19 lattice discretization scheme will be used, which introduces 19 discrete particle velocities in directions $\mathbf{e}_{i}$ defined as, $\boldsymbol{e}_{i}=c .\left\{\begin{array}{c|cccccc|cccccccccccc}0 & 1 & -1 & 0 & 0 & 0 & 0 & 1 & -1 & 1 & -1 & 1 & -1 & 1 & -1 & 0 & 0 & 0 & 0 \\ 0 & 0 & 0 & 1 & -1 & 0 & 0 & 1 & -1 & -1 & 1 & 0 & 0 & 0 & 0 & 1 & -1 & 1 & -1 \\ 0 & 0 & 0 & 0 & 0 & 1 & -1 & 0 & 0 & 0 & 0 & 1 & -1 & -1 & 1 & 1 & -1 & -1 & 1\end{array}\right\}$

where $c=\Delta x / \Delta t$ denotes the particle propagation speed on the lattice [15], and $\Delta x$ and $\Delta t$ are the lattice constant mesh size and time step, respectively.

Details of the LBM equations and schemes are given in the following sections. 


\subsubsection{Lattice Boltzmann solution of NS equations}

The macroscopic continuity and momentum (i.e., NS) equations for incompressible Newtonian fluids read (using the index summation convention),

$$
\begin{aligned}
\partial_{\alpha} u_{\alpha} & =0 \\
\partial_{t} u_{\alpha}+\partial_{\beta}\left(u_{\alpha} u_{\beta}\right)= & -\frac{1}{\rho} \partial_{\beta}(p)+\partial_{\beta}\left\{\mu\left(\partial_{\alpha} u_{\beta}+\partial_{\beta} u_{\alpha}\right)\right\} \\
& +\frac{k}{\rho} \partial_{\beta}\left\{\frac{\partial \phi}{\partial x_{\gamma}} \frac{\partial \phi}{\partial x_{\gamma}} \delta_{\alpha \beta}-\frac{\partial \phi}{\partial x_{\alpha}} \frac{\partial \phi}{\partial x_{\beta}}\right\}
\end{aligned}
$$

where the last term in Eq. 2.3) is non-standard and represents surface tension effects at the diffuse interface between both fluids [5] ( $k$ is related to the $\phi_{1}$ and $\phi_{2}$ and the surface tension coefficient $\sigma_{12}$, for fluids 1 and 2 , and will be defined in Section 2.2.3). These equations are solved in a LBM framework using the particle distribution functions $g_{i}$, whose time evolution is governed by,

$$
g_{i}\left(\boldsymbol{x}+\boldsymbol{e}_{i} \Delta t, t+\Delta t\right)=g_{i}(\boldsymbol{x}, t)-\frac{\Delta t}{\tau_{g}}\left(g_{i}(\boldsymbol{x}, t)-g_{i}^{(e q)}(\boldsymbol{x}, t)\right)+\Delta t\left(F_{i}+G_{i}\right)
$$

where $F_{i}$ and $G_{i}$ represent effects of the viscous and gravitational volume forces, respectively, $\tau_{g}$ is a relaxation time, and $g_{i}^{e q}$ are the equilibrium distribution functions defined as,

$$
\begin{aligned}
g_{i}^{(e q)}= & w_{i}\left\{\frac{e_{i \alpha} u_{\alpha}^{*}}{c_{s}^{2}}+\frac{\left(e_{i \alpha} u_{\alpha}^{*}\right)^{2}}{2 c_{s}^{4}}-\frac{\left|\boldsymbol{u}^{*}\right|^{2}}{2 c_{s}^{2}}+\frac{1}{2 c_{s}^{2}}\left(\tau_{g}-\frac{1}{2} \Delta t\right)\left(\frac{\partial u_{\beta}}{\partial x_{\alpha}}+\frac{\partial u_{\alpha}}{\partial x_{\beta}}\right) e_{i \alpha} e_{i \beta}\right\} \\
& +w_{i} \frac{k}{\rho} T_{\alpha \beta} e_{i \alpha} e_{i \beta}-v_{i} \frac{k}{2 \rho}|\nabla \phi|^{2} .
\end{aligned}
$$

where $c_{s}=c / \sqrt{3}$, is the speed of sound and,

$$
\begin{aligned}
w_{0} & =\frac{1}{3} ; w_{1, \ldots, 6}=\frac{1}{18} ; w_{7, \ldots, 18}=\frac{1}{36}, \\
v_{0} & =-\frac{2}{c^{2}} ; v_{i}=\frac{3}{c^{2}} w_{i}(i=1,2, \ldots, 18),
\end{aligned}
$$

are LBM weights used for the D3Q19 scheme, and,

$$
T_{\alpha \beta}(\phi)=\frac{9}{2 c^{4}} \frac{\partial \phi}{\partial x_{\alpha}} \frac{\partial \phi}{\partial x_{\beta}}-\frac{3}{2 c^{4}} \frac{\partial \phi}{\partial x_{\gamma}} \frac{\partial \phi}{\partial x_{\gamma}} \delta_{\alpha \beta} .
$$


expresses surface tension effects at the two-fluid interface, together with the last term in Eq. (2.5). The last term in the curly bracket in Eq. (2.5) removes the dependency of $\tau_{g}$ on viscosity, which makes the proposed method extremely stable for the simulation of high Reynolds number flows. For this reason, viscous effects are added back as a volume force in the evolution Eq. (2.4), together with the effect of the gravity force. The volume forces expressing the contributions of viscous effects and gravity force in the LBM governing equation read,

$$
\begin{aligned}
F_{i} & =\left(1-\frac{\Delta t}{2 \tau_{g}}\right) w_{i}\left[\frac{\boldsymbol{e}_{i}-\boldsymbol{u}}{c_{s}^{2}}+\frac{\boldsymbol{e}_{i} \cdot \boldsymbol{u}}{c_{s}^{4}} \boldsymbol{e}_{i}\right] \cdot \boldsymbol{F} \\
G_{i} & =\left(1-\frac{\Delta t}{2 \tau_{g}}\right) w_{i}\left[\frac{\boldsymbol{e}_{i}-\boldsymbol{u}}{c_{s}^{2}}+\frac{\boldsymbol{e}_{i} \cdot \boldsymbol{u}}{c_{s}^{4}} \boldsymbol{e}_{i}\right] \cdot \boldsymbol{G}
\end{aligned}
$$

with $\boldsymbol{G}$ the gravitational acceleration vector, and $\boldsymbol{F}=\frac{1}{\rho} \partial_{\beta}\left(\mu\left(\frac{\partial u_{\beta}}{\partial x_{\alpha}}+\frac{\partial u_{\alpha}}{\partial x_{\beta}}\right)\right)$ the viscous stress per unit mass. Note that the extended body force formulation of Guo et al. [16] has been used in these equations, since body forces vary in time and space.

The presence of density in the standard equilibrium distribution functions used for solving NS equations is the main source of instability identified in various earlier LBM solutions of multiphase flows with high density ratios [13, 17]. Here, to eliminate these instabilities, following Banari et al. [5], density was removed from the first part of the equilibrium functions in Eq. (2.5). This will require adding a correction to the velocity field to satisfy mass conservation, as discussed next. It can be shown by applying a Chapman-Enskog expansion that Eq. (2.4) with the equilibrium distribution functions defined in Eq. 2.5 converges to the NS equations (Eqs. 2.3), 2.2) , without the pressure gradient term (referred to as "pressureless" NS equations). The details of the Chapman-Esnkog expansion are shown in Appendix A. The fluid velocity corresponding to these pressureless NS 
equations is found from Eq. (2.5) as,

$$
u_{\alpha}^{*}=\sum_{i=0}^{b} g_{i} e_{i \alpha}+\frac{\Delta t}{2}(\boldsymbol{F}+\boldsymbol{G}) \text {, }
$$

However, this velocity is not divergence free and hence needs to be corrected. Thus, similar to [5, 9], the corrected velocity $\boldsymbol{u}$ is obtained as,

$$
\boldsymbol{u}=\boldsymbol{u}^{*}+\Delta \boldsymbol{u}
$$

with the correction $\Delta \boldsymbol{u}$ defined as,

$$
\Delta \boldsymbol{u} \simeq-\Delta t \frac{\nabla p}{\rho}
$$

as a function of the gradient of the pressure $p$, which is obtained by solving the following Poisson equation,

$$
\nabla \cdot\left(\frac{\Delta t \nabla p}{\rho}\right)=\nabla \cdot \boldsymbol{u}^{*}
$$

Similar to [5], this equation is solved iteratively in a LBM framework. For this purpose, an additional set of particle distribution functions $h_{i}(i=0, \ldots, 18)$ is introduced, whose time evolution is governed by,

$$
h_{i}^{n}\left(\boldsymbol{x}+\boldsymbol{e}_{i} \Delta t, t+\Delta t\right)=h_{i}^{n}(\boldsymbol{x}, t)-\frac{\Delta t}{\tau_{h}}\left(h_{i}^{n}-h_{i}^{(e q, n)}\right)-\frac{1}{3} w_{i}\left(\nabla \cdot \boldsymbol{u}^{*}(t)\right)
$$

where $n$ denotes the $n$-th iteration in the Poisson equation solution (see [9] for details). In this second LBM scheme, the equilibrium distribution functions are defined as,

$$
h_{i}^{(e q, n)}=\frac{w_{i} p^{n}(\boldsymbol{x}, t)}{\rho_{0} c^{2}}
$$

with $\rho_{0}$ denoting a reference density and the relaxation time $\tau_{h}$ being related to the density by,

$$
\tau_{h}=\Delta t\left(\frac{\rho_{0} c^{2}}{\rho c_{s}^{2}}+\frac{1}{2}\right) .
$$


Once the equations 2.15) to 2.17) are solved for a given iteration $n$, the pressure is obtained as the zero-th order moment of the particle distribution functions as,

$$
p^{n+1}=\rho_{0} c^{2} \sum_{i=0}^{b} h_{i}^{n} .
$$

This scheme is solved iteratively until convergence is achieved on the pressure for a given time step. The velocity correction is calculated using Eq. (2.13).

As shown in the Chapman-Enskog expansion (Appendix A), the complete set of LBM equations (2.4) to (2.18) solves the complete set of NS equations (2.3). Unlike the classical LBM, however, the relaxation time $\tau_{g}$ can be chosen arbitrarily, independently from the Reynolds number, and the scheme is stable for

high density ratios between fluids. It should be pointed out that, incorporating viscous forces as volume forces creates additional spurious terms in the macroscopic NS equation. These terms, however, scale with viscosity and hence are negligible for high Reynolds number flows [5].

\subsubsection{Lattice Boltzmann scheme for interface capturing}

In this 3D model, similar to the 2D model of Banari et al. [5], the position of the interface between the 2 fluids is tracked using the Cahn-Hilllard advectiondiffusion equation,

$$
\partial_{t}(\phi)+\partial_{\alpha}\left(\phi u_{\alpha}\right)=M \nabla^{2} \mu_{\phi}
$$

where $M$ is the mobility parameter between two components, and $\mu_{\phi}$ is the chemical potential (see details below). Diffuse interface schemes offer some advantages as compared to sharp interface schemes, for which fluid parameters such as density and viscosity vary discontinuously across the interface. Such discontinuities often cause numerical noise, which can potentially trigger instabilities, especially for high density and viscosity ratios. By contrast, in diffuse interface schemes, fluid 
properties continuously and smoothly vary over a short distance across the interface. Additionally, for sharp interface schemes, the accurate computation of the interface curvature and related surface tension forces is very challenging, whereas surface tension effects are intrinsically included in diffuse interface models. Here, similar to [5], the motion of the diffusive interface is modeled by the Cahn-Hilliard equation,

In the model, the chemical potential is defined as,

$$
\mu_{\phi}=\frac{d \Psi}{d \phi}-k \nabla^{2} \phi \quad \text { with } \quad \Psi(\phi)=\left(\phi-\phi_{2}\right)^{2}\left(\phi-\phi_{1}\right)^{2}
$$

the bulk free-energy density. Coefficients $k$ and $\beta$ are related to the surface tension coefficient $\sigma_{12}$ and interface thickness $W$ by,

$$
W=\frac{4}{\phi_{1}-\phi_{2}} \sqrt{\frac{k}{2 \beta}} \quad \text { and } \quad \sigma_{12}=\frac{\left(\phi_{1}-\phi_{2}\right)^{3}}{6} \sqrt{2 k \beta}
$$

A more detailed derivation and discussion of this model can be found in [5].

Equations 2.19 to 2.21), are solved with a LBM using a third set of probability distribution functions, $f_{i}(x, t)$, whose time evolution is governed by,

$$
f_{i}\left(\boldsymbol{x}+\boldsymbol{e}_{i} \Delta t, t+\Delta t\right)=f_{i}(\boldsymbol{x}, t)-\frac{\Delta t}{\tau_{f}}\left(f_{i}(\boldsymbol{x}, t)-f_{i}^{(e q)}(\boldsymbol{x}, t)\right) .
$$

The corresponding equilibrium distribution functions $f_{i}^{(e q)}$ read,

$$
f_{i}^{(e q)}=H_{i} \phi+v_{i} \frac{M}{\tau_{f}-\frac{1}{2} \Delta t} \mu_{\phi}+\phi w_{i}\left\{\frac{e_{i \alpha} u_{\alpha}}{c_{s}^{2}}+\frac{\left(e_{i \alpha} u_{\alpha}\right)^{2}}{2 c_{s}^{4}}-\frac{|\boldsymbol{u}|^{2}}{2 c_{s}^{2}}\right\},
$$

with,

$$
H_{0}=1 \quad \text { and } \quad H_{1,2, \ldots, 18}=0
$$

and $w_{i}$ and $v_{i}$ defined as in Eqs. 2.6 and 2.7). The order parameter $\phi$ is computed from the first moment of the distribution functions as,

$$
\phi=\sum_{i=0}^{b} f_{i}
$$


Once the order parameter computed, the spatial distribution of fluid properties (density $\rho$ and dynamic viscosity $\mu$ ) in the entire domain, and in particular across the interface can be expressed as,

$$
\rho(\phi)= \begin{cases}\rho_{2} & \phi \leq \phi_{2} \\ \frac{\phi-\phi_{2}}{\phi_{1}-\phi_{2}}\left(\rho_{1}-\rho_{2}\right)+\rho_{2} & \phi_{2}<\phi<\phi_{1} \\ \rho_{1} & \phi \geq \phi_{1} .\end{cases}
$$

Rather than directly expressing the kinematic viscosity as a function of $\phi$, it is defined as a function of density as,

$$
\mu(\rho)=\frac{\rho-\rho_{2}}{\rho_{1}-\rho_{2}}\left(\mu_{1}-\mu_{2}\right)+\mu_{2} .
$$

This was found by Banari et al. [5] to yield more accurate results. 


\subsubsection{Numerical implementation and performance}

The numerical algorithm for this multi-component LBM model is given in Algorithm 3. The entire model is implemented for a parallel solution on a GPGPU, using the CUDA language in the NVIDIA environment. In the algorithm, after initializing all the fluid properties and LBM variables, these are adjusted through initial iterations performed on the interface shape or the field variables to fulfill the governing equations. Specifically, the initially sharp interface between the two fluids specified at time step zero is smoothed out by solving the advection-diffusion equation, for the interface capturing, for a fixed number of initial iterations (e.g., 5000), while assuming a zero velocity field and adjusting interface thickness based on Eq. 2.21). Once physically relevant initial conditions are achieved, the main computational time loop is started. Note that, in the latter, to increase the efficiency of the computations, the convergence of the iterative solution of the Poisson equation is only checked every 20 iterations.

The performance of our new 3D LBM multiphase model highly depends on the number of Poisson iterations performed at each time step, which depends upon the problem physics that is tackled. For a given relative error threshold $\varepsilon$, we find that the number of iterations required to solve the Poisson equation is initially larger, in the first few time steps of calculations, while the field variables are still dynamically adjusting to the governing equations, but then decreases later on. After this early phase of computations we can evaluate the model efficiency in terms of the usual metric used in LBM models, the "Million of Node Updates Per Second" (MNUPS). On a NVIDIA TESLA 2070 GPGPU (448 CUDA cores), the performance of the current scheme is found to be around 20 MNUPS, using the largest possible grid that can fit in he 6Gb RAM, with about $40 \%$ of the 
Algorithm 3 Algorithm for the LBM computation of two-fluid flow fields and phase field interface update

Set initial conditions, $\phi=\phi_{i}, \rho=\rho_{i}, \mu=\mu_{i}$ and $p=0$

Initialize particle distribution functions, i.e. set

$f_{i}\left(\boldsymbol{x}, t_{0}\right)=f_{i}^{e q}\left(\boldsymbol{x}, t_{0}\right), g_{i}\left(\boldsymbol{x}, t_{0}\right)=g_{i}^{e q}\left(\boldsymbol{x}, t_{0}\right), h_{i}\left(\boldsymbol{x}, t_{0}\right)=h_{i}^{e q}\left(\boldsymbol{x}, t_{0}\right)$

Run initial iterations to improve the initial conditions for the phase field

for $t<t_{\text {end }}$ do

Compute $f_{i}(\boldsymbol{x}, t+\Delta t)$ using Eq. 2.22

Compute $g_{i}(\boldsymbol{x}, t+\Delta t)$ using Eq. (2.4),

Compute $\phi(\boldsymbol{x}, t+\Delta t)$ and $\boldsymbol{u}^{*}(\boldsymbol{x}, t+\Delta t)$ with Eqs. (2.25) and (2.11);

$\rho(\boldsymbol{x}, t+\Delta t)$ and $\mu(\boldsymbol{x}, t+\Delta t)$ are calculated using Eqs. (2.26) and (2.27).

For every 20 Poisson iterations:

if $\left|\frac{p^{n+1}-p^{n}}{p^{n}}\right|>\varepsilon$ then

Compute $p^{n+1}(\boldsymbol{x}, t+\Delta t)$ using Eq. (2.15)-(2.18).

end if

Compute $\boldsymbol{u}(\boldsymbol{x}, t+\Delta t)$ using Eq. 2.13).

end for

computational time spent for solving the Poisson equation. While this is less than for standard LBM schemes applied to a single fluid, which may achieve over $100 M N U P S$, this is still a very respectable performance considering the higher complexity of solving large Reynolds number flows, for two fluids with a high density ratio, while tracking their 3D interface, which may have a very complex geometry in some applications (e.g., ocean breaking waves).

\section{$2.3 \quad$ Validation}

A few increasingly complex validation/benchmarking applications of our LBM scheme are presented in the following, to assess the accuracy and convergence of the presented multiphase model and confirm its relevance from investigating new physics in multi-fluids flows, particularly in the context of air-sea interactions, which is the motivation for this research. All simulations are carried out in a dimensionless framework, using non-dimensional lattice variables (here denoted 
by a prime) scaled on the basis of a length scale $\lambda$, time scale $\tau$ and mass scale $\varpi$. Thus, for the mesh parameters, $\Delta x^{\prime}=\Delta x / \lambda, \Delta t^{\prime}=\Delta t / \tau$ and $c^{\prime}=c \tau / \lambda$. It is also customary in LBM to assume that $c^{\prime}=1$, which is akin to having the mesh Courant number be unity. If the length scale is further defined as $\lambda=\Delta x$, we then have $\Delta x^{\prime}=1$ and this requires $\tau=\Delta t$ and $\Delta t^{\prime}=1$ as well. Hence, with these definitions, in lattice variables, we always have $c^{\prime}=\Delta x^{\prime}=\Delta t^{\prime}=1$ and $c_{s}^{\prime}=c^{\prime} / \sqrt{3}=1 / \sqrt{3}$ [15. Accordingly, the dimensionless relaxation times are defined as,

$$
\tau_{g}^{\prime}=\frac{\tau_{g}}{\Delta t} \quad \text { and } \quad \tau_{h}^{\prime}=\frac{1}{2}+\frac{\rho_{0}^{\prime}}{\rho^{\prime}}
$$

for distribution functions $g_{i}$ and $h_{i}$, respectively, with $\rho^{\prime}=\rho \lambda^{3} / \varpi$ and $\rho_{0}^{\prime}=$ $\rho_{0} \lambda^{3} / \varpi$. The non-dimensional reference density is set to $\rho_{0}^{\prime}=1$, which implies that, $\varpi=\rho_{0}(\Delta x)^{3}$ (usually, one will also assume for simplicity, $\rho_{0}=1 \mathrm{~kg} / \mathrm{m}^{3}$ ) and $\rho_{i}^{\prime}=\rho_{i}$, for fluid $i=1,2$. The non-dimensional surface tension coefficient is further defined as,

$$
\sigma_{12}^{\prime}=\frac{\sigma_{12} \tau^{2}}{\rho_{0} \lambda^{3}}=\frac{\sigma_{12}}{\rho_{0} c^{2} \Delta x}
$$

For the phase order function, we specify $\phi_{1}=1.0$ and $\phi_{2}=0.0$ in all cases, and the interface thickness is assumed to span 4 lattice meshes, i.e., $W=4 \Delta x$ or $W^{\prime}=4$, which with Eq. 2.21 yields the interface lattice parameters,

$$
\beta^{\prime}=\frac{3 \sigma_{12}^{\prime}}{\left(\phi_{1}-\phi_{2}\right)^{4}} \quad \text { and } \quad k^{\prime}=\frac{6 \sigma_{12}^{\prime}}{\left(\phi_{1}-\phi_{2}\right)^{2}}
$$

where $\beta^{\prime}=\beta /\left(\rho_{0} c^{2}\right)$ and $k^{\prime}=k /\left(\rho_{0} c^{2} \lambda^{2}\right)$. The error threshold for the Poisson equation is set to $\varepsilon=10^{-5}$ for all simulations. Additionally, the non-dimensional relaxation time for solving the Cahn-Hilliard Eq. 2.22 is set to $\tau_{f}^{\prime}=\tau_{f} / \Delta t=1$, and the non-dimensional mobility coefficient, $M^{\prime}=M \varpi /\left(\tau \lambda^{3}\right)$ is set to $M^{\prime}=$ $0.001 / \beta^{\prime}$. It should be pointed out that Lee et al. [11] suggested $M^{\prime}=0.02 / \beta^{\prime}$ 
to get the most stable results, but such a large mobility would lead to additional diffusion in the fluid. Hence, we selected a lower mobility coefficient to prevent such an unrealistic diffusion. The relaxation time $\tau_{g}^{\prime}$ is set to unity in all cases, because this is a suitable choice to ensure that LBM simulations are numerically stable. In the following, our LBM model will be successively applied to the simulation of: (i) a droplet impacting a thin wet surface; (ii) a bubble rising in a quiescent heavier fluid; and (iii) a breaking ocean wave.

\subsubsection{Droplet impact on a thin wet surface}

Simulating the impact of a droplet on a wet surface is important for a wide variety of engineering and industrial design problems, such as turbine blades, ink-jet printing, and internal combustion engines. Worthington was the first to study the droplet splash in 1908 [18]. However, more than a century later, this phenomenon is still far from being fully understood, due to the complexity of the problem. The important parameters physical this problem are: (i) the direction of impact (normal or oblique); (ii) the depth of the fluid layer; (iii) the viscosity and density ratios of the two fluids; (iv) whether these are Newtonian or non-Newtonian fluids; (v) the droplet velocity at the time of impact; and (vi) the importance of gravitational effects. Here, for simplicity, we investigate the impact of a single droplet on a thin layer of the same fluid, only up to a short time after the impact. Earlier experi-

mental work [19, 20] shows that the impact of a droplet on a thin liquid film may follow two patterns: (i) splashing; and (ii) spreading (deposition). In the spreading pattern, the impact velocity is low and the droplet spreads over the wall, taking the shape of lamella, whereas in the splashing pattern, the impact velocity is high and the lamella eventually takes the shape of a crown consisting of a thin liquid sheets with an unstable free rim at the top, from which numerous small secondary droplets are ejected (Rayleigh-Plateau instability). The important dimensionless 
numbers relevant for the droplet impact problem are the Weber number and the Reynolds number, which based on, e.g., the properties of fluid 1, read,

$$
\mathrm{We}=\frac{2 R U^{2} \rho_{1}}{\sigma_{12}} \quad \text { and } \quad \operatorname{Re}=\frac{2 R U}{\nu_{1}}
$$

where $U$ is the impact velocity (velocity when the droplet touches the fluid film), $\rho_{1}$ and $\nu_{1}$ are the density and kinematic viscosity of fluid 1, respectively, $R$ is the initial radius of the droplet and $\sigma_{12}$ is the surface tension for the two fluids. Dimensional analysis shows that the gravity force is negligible in the droplet impact and therefore this term $\left(G_{i}\right)$ is not kept in the LBM equations for this application.

We simulate the fall of a 3D droplet made of fluid 1 in a second lighter fluid (fluid 2), concluded by the droplet impact on a thin layer of fluid 1. Fig. 2.1 shows the computed time evolution of the droplet impact where, owing to symmetry in the $x$ direction, the solution in only half of the domain was calculated. The grid size is $128 \times 256 \times 64$ in the $x, y$, and $z$ directions, respectively. The density ratio is $\rho_{1} / \rho_{2}=1000$ and the viscosity ratio is $\mu_{1} / \mu_{2}=100$ (such as for water (1) and air (2)). The Reynolds number is $R e=200$, with $U=0.01 \Delta x / \Delta t$ and the Weber number is $W e=8000$.

Earlier work [19, 20] indicates that the droplet spreading radius $r$ should vary with time as $\sqrt{2 R U t}$ during the splashing process (power law). To confirm this, the dimensionless spreading factor $(r / 2 R)$ is plotted versus the non-dimensional time $(U t / 2 R)$ in Figure 2.2. Our numerical results are found to follow the power law, except for a short time after the impact, which could result from the impulsive start of the collision, which does not occur in experiments.

A further validation of our numerical results is performed for the impact of a droplet on a thin layer of fluid by comparing with Lee et al.'s [8] numerical results, which were $2 \mathrm{D}$. In our $3 \mathrm{D}$ model, a grid with $1024 \times 512 \times 2$ points is used in the numerical simulations, where the 2 points across the width are aimed at 
representing $2 \mathrm{D}$ conditions. The domain set-up is shown in Fig. 2.3, symmetry boundary conditions are specified on the leftward and rightward boundaries, and no-slip boundary conditions on the top and bottom walls; periodic boundary conditions are specified along the third dimension. As in Lee et al., the density ratio is set to $\rho_{1} / \rho_{2}=1,000$ and the viscosity ratio is adjusted to $\mu_{1} / \mu_{2}=40$. The Weber number is set to $W e=8,000$, the droplet diameter is $R=60 \Delta x$, the layer thickness is such that $H / R=0.25$, and the impact velocity is $U=.005 \Delta x / \Delta t$. Four different simulations were performed for four different Reynolds numbers, $R e=20,100,500,2000$.

Figs. 2.4, 2.5, 2.6) show the computed time evolution, for a few nondimensional times $U t / 2 R$ values, of the droplet shape after impact in the first three cases, compared to Lee et al.'s [8] results. Overall, we observe a very good agreement for all three configurations with Lee et al.'s results. In Figure 2.4, the Reynolds number is low and deposition patterns occur. Viscous forces dominate the initial phase of the impact and the droplet gently spreads on the surface without splashing at all. Immediately after impact, a certain amount of the fluid 1 is trapped within fluid 2, and small bubbles are formed, due to effects of surface tension. The smaller bubbles diffuse into fluid 2 and eventually disappear, after they become smaller than the grid resolution, while the larger ones (Figure 2.4. e/f) persist and advect with the flow.

In Figs. (2.5) and (2.6) the impact process occurs for higher Reynolds numbers $R e=100,500$, for which droplet inertia is sufficient to overcome the viscous forces, causing the creation of a finger of fluid 1 into fluid 2. This finger is not stable and eventually breaks up into smaller droplets. This process, also referred to as Rayleigh-Plateau instability, can be clearly observed in Figs. 2.5 and 2.6 (f), whereas this instability was not observed in the work of Lee et al. 
Finally, results for a droplet impacting a fluid layer at an even higher Reynolds number, $\operatorname{Re}=2,000$, are shown in Fig. 2.7. Note that the method of Lee et al. was not able to go beyond $R e=100$ (based on the grid size they used), because of the dependence of relaxation time on viscosity, which has been removed in the current work. 


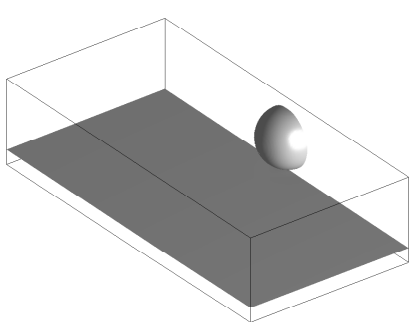

(a) $U t / 2 R=0.0$

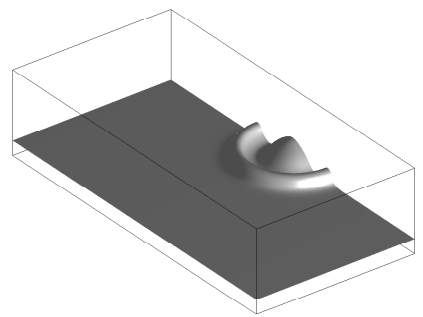

(c) $U t / 2 R=1.125$

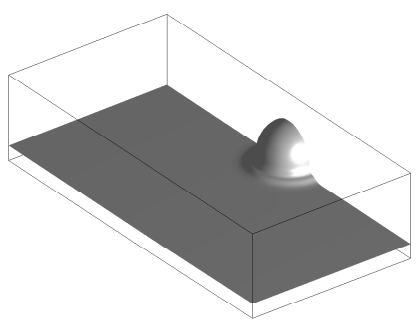

(b) $U t / 2 R=0.5$

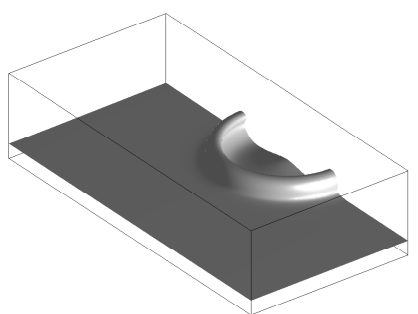

(d) $U t / 2 R=1.8$

Figure 2.1: Time evolution of the 3D simulation of a droplet impacting a thin layer of fluid, at four different times $t$, for $\rho_{1} / \rho_{2}=1000, \mu_{1} / \mu_{2}=100, R e=200$ and $W e=8000$.

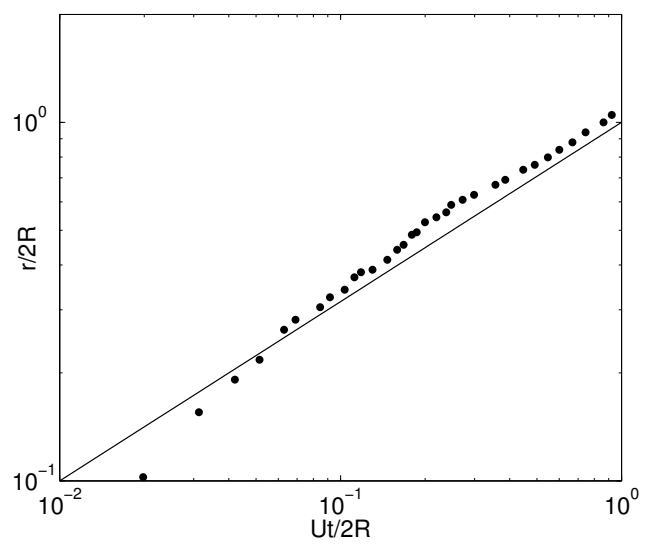

Figure 2.2: Spread factor $r / 2 R$ as a function of $U t / 2 R$ in the $3 \mathrm{D}$ simulation of a droplet impacting a thin layer of fluid, for $\rho_{1} / \rho_{2}=1000, \mu_{1} / \mu_{2}=100, R e=200$ and $W e=8,000$. The straight line corresponds to the power law $r=\sqrt{2 U t R}$. 


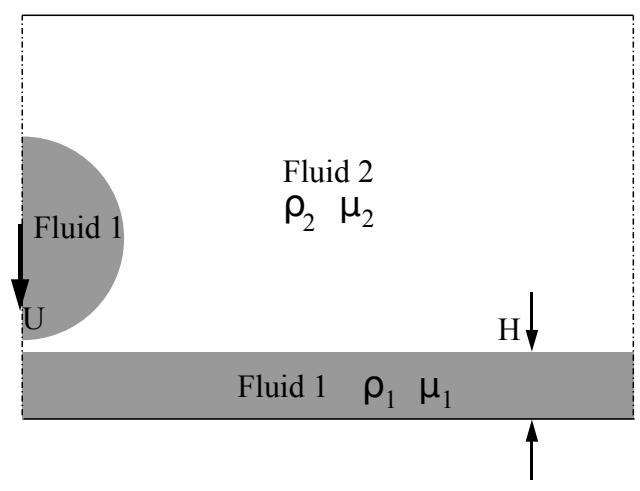

Figure 2.3: Domain and parameter definition for the quasi-2D simulation of a droplet impacting a thin fluid layer, following Lee et al. [8].
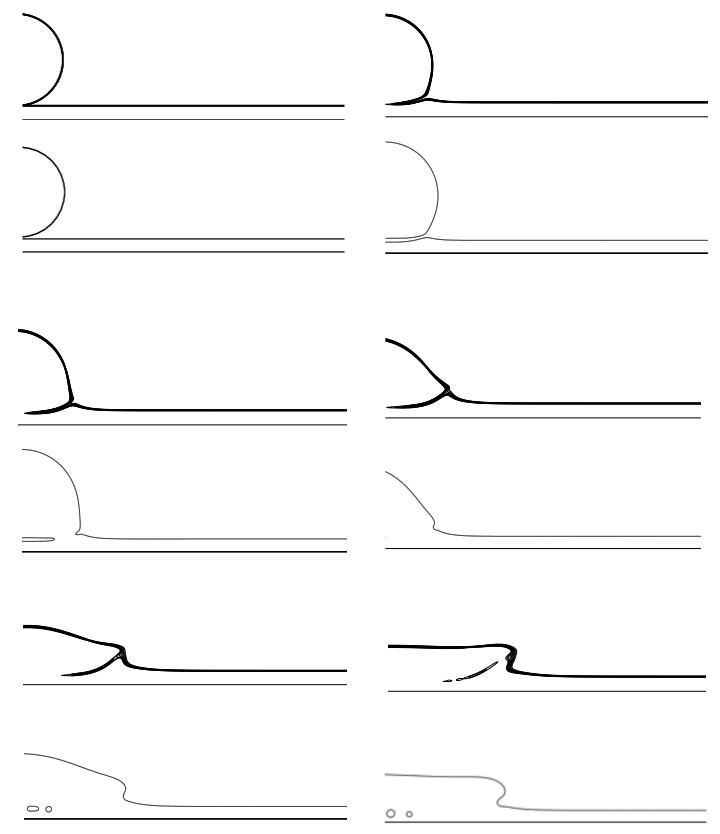

Figure 2.4: Time evolution in quasi-2D simulations of a droplet impacting a thin fluid film for $\mathrm{Ut} / 2 \mathrm{R}=0.0,0.1,0.2,0.4,0.8,1.6 . R e=20, W e=8000, \rho_{1} / \rho_{2}=1000$ and $\nu_{1} / \nu_{2}=40$; top: Lee et al.'s results [8], bottom: current LBM model. 

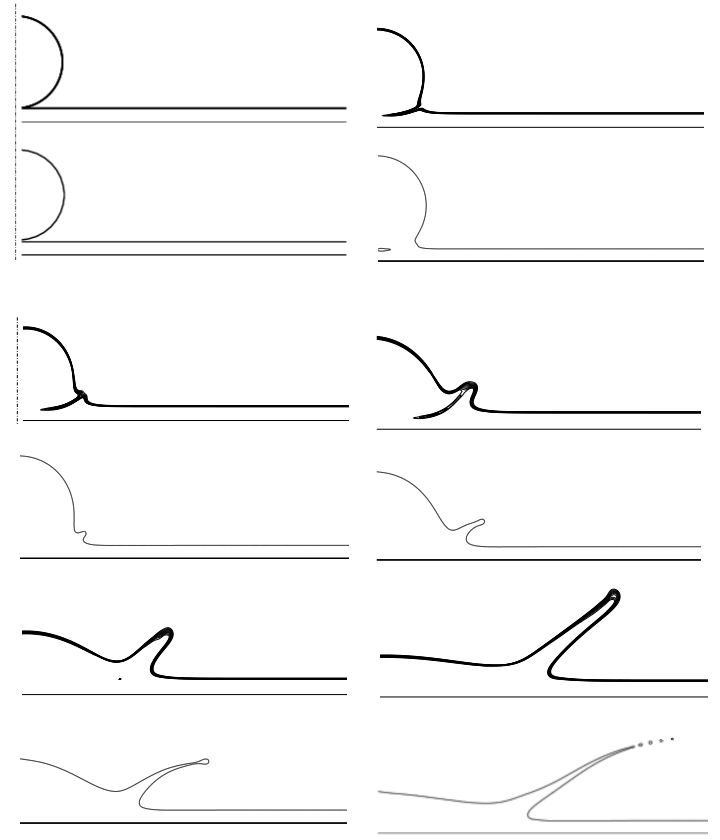

Figure 2.5: Time evolution in quasi-2D simulations of a droplet impacting a thin fluid film for $\mathrm{Ut} / 2 \mathrm{R}=0.0,0.1,0.2,0.4,0.8,1.6 . R e=100, W e=8000, \rho_{1} / \rho_{2}=$ 1000 and $\nu_{1} / \nu_{2}=40$; top: Lee et al.'s results [8], bottom: current LBM model. 


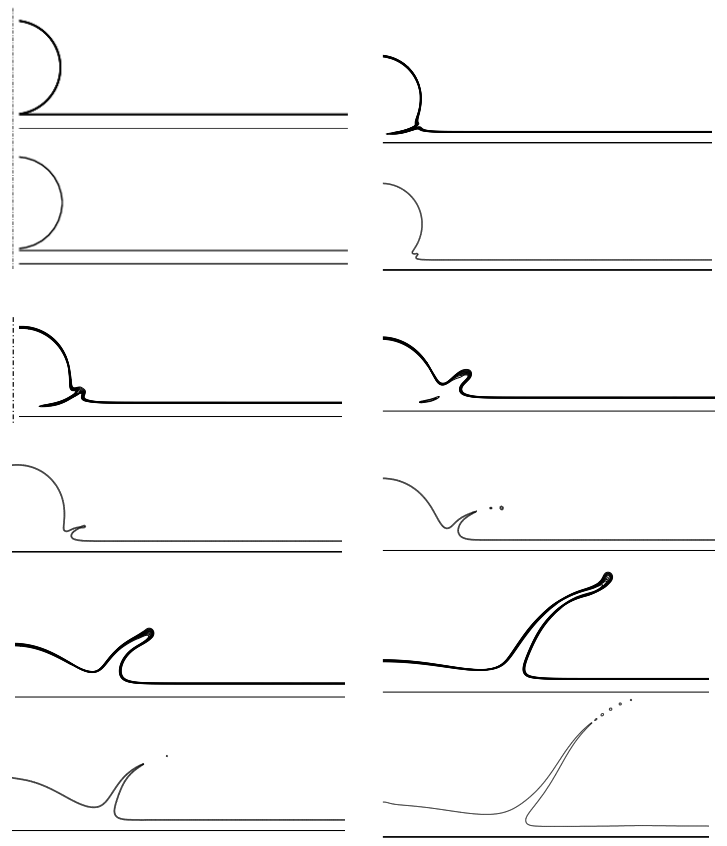

Figure 2.6: Time evolution in quasi-2D simulations of a droplet impacting a thin fluid film for $\mathrm{Ut} / 2 \mathrm{R}=0.0,0.1,0.2,0.4,0.8,1.6 . R e=500, W e=8000, \rho_{1} / \rho_{2}=$ 1000 and $\nu_{1} / \nu_{2}=40$; top: Lee et al.'s results [8], bottom: current LBM model. 


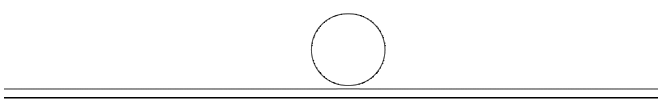

(a) $U t / 2 R=0.0$

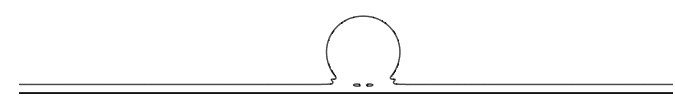

(b) $U t / 2 R=0.1$

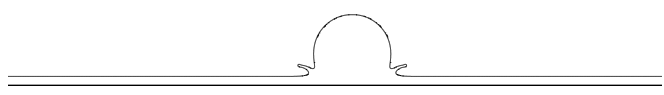

(c) $U t / 2 R=0.2$

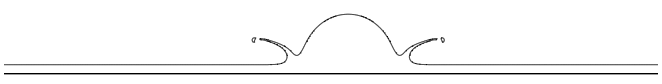

(d) $U t / 2 R=0.4$

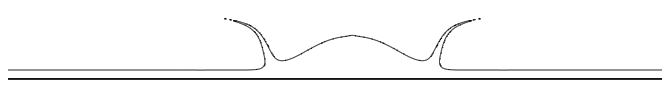

(e) $U t / 2 R=0.8$

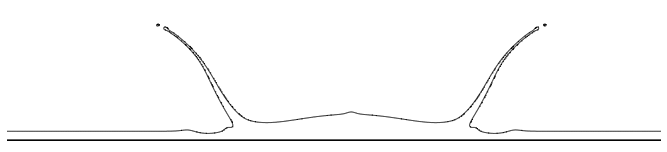

(f) $U t / 2 R=1.6$

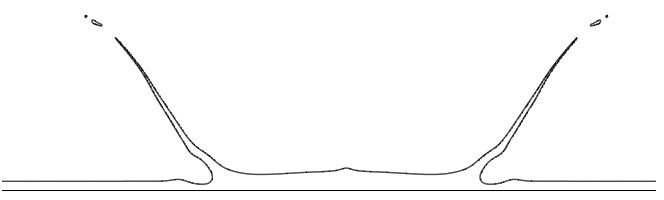

(g) $U t / 2 R=2.4$

Figure 2.7: Time evolution in quasi-2D simulations of a droplet impacting a thin fluid film, for $R e=2000, W e=8000, \rho_{1} / \rho_{2}=1000$ and $\nu_{1} / \nu_{2}=40$, using current LBM model. 


\subsubsection{Rising bubble in quiescent fluid}

Here, we model the dynamic behavior of a 3D bubble of a light fluid $\left(\rho_{2}, \nu_{2}\right)$ rising in a heavier fluid $\left(\rho_{1}, \nu_{1}\right)$, due to the buoyancy force. Although the LBM simulation setup in terms of grid initialization and boundary conditions is straightforward, the flow structure around the bubble is quite complex and governed by competing effects of viscosity, buoyancy, and surface tension forces. Several experimental studies have been conducted to measure the rise and deformation of single bubbles in a quiescent fluid [21, 22]. These showed that the bubble shape greatly varies according to various flow regimes defined by non-dimensional parameters, such as the Bond number Bo (also known as Eotvos number, the ratio of gravity to surface tension forces), the Reynolds number Re, and the Morton number $M o$, defined as,

$$
B o=\frac{g D^{2}}{\sigma_{12}}\left(\rho_{1}-\rho_{2}\right) \quad ; \quad M o=\frac{g \mu_{1}^{4}}{\sigma_{12}^{3} \rho_{1}}\left(1-\frac{\rho_{2}}{\rho_{1}}\right) \quad ; \quad R e=\frac{\rho_{1} U D}{\mu_{1}}
$$

with the bubble terminal velocity $U$ and diameter $D$, the gravitational acceleration $g$, and the dynamic viscosity of the heavier fluid $\mu_{1}$. The terminal shapes of individual rising bubbles were experimentally measured for a broad range of Reynolds and Bond numbers [21], and can be categorized into three shape regimes: (a) spherical, (b) ellipsoidal, (c) spherical cap. In the spherical regime, for small $B o$ values, surface tension is dominant and prevents the deformation of the bubble to occur under inertia and viscous forces; consequently, the shape of the bubble remains (almost) spherical during its rise. When increasing the Reynolds and Bond numbers, the contribution of surface tension gradually becomes less important as compared to inertia, and the terminal shape of the bubble becomes ellipsoidal for moderate Reynolds and Bond numbers $(10<R e<500$ and $10<B o<100)$, and spherical cap for high Reynolds and Bond numbers.

In our 3D LBM simulations, a spherical bubble of density $\rho_{2}^{\prime}=1$ and initial 
diameter $D_{0}^{\prime}=32$ is placed at one bubble diameter above the bottom of a domain of size $4 D^{\prime} \times 4 D^{\prime} \times 8 D^{\prime}$, filled with a fluid of density $\rho_{1}^{\prime}=1000$ (hence the $\rho_{1}^{\prime} / \rho_{2}^{\prime}=$ 1000); the fluid viscosity ratio is $\mu_{1}^{\prime} / \mu_{2}^{\prime}=100$, which approximates the air-water case. Amaya et al. [23] reported that wall effects are negligible when a domain size of $4 D^{\prime}$ or larger in the radial direction is used. Also, the vertical domain size has to be large enough to allow the bubble to adjust to its final steady shape during its rise. The required distance of this to occur is different for each case and depends on the Reynolds number, which directly controls the bubble rising velocity. For cases up to $R e \sim 100$, a domain of height $8 D^{\prime}$ was found to be sufficient.

Initially, both fluids are stationary; free slip boundary conditions are prescribed on the lateral sides of the domain and a bounce-back condition on the top and bottom boundaries. Simulations are run for four distinct test cases (A to D) with different $M o$ and $B o$ values. In Table 2.1, the terminal Reynolds number is compared with experimental data and a reasonable agreement between numerical and experimental results can be observed, but there are larger discrepancies for case A. Similar larger errors for this case were reported in the work of Hua et al. [24], which they interpreted as being due the extremely low rising velocity leading to higher relative errors.

\begin{tabular}{cccccc}
\hline Test case & Mo & Bo & Simulated Re & Experimental Re & Relative error on Re (\%) \\
\hline A & 711 & 17.7 & 0.189 & 0.232 & 18.53 \\
B & $8.2 \times 10^{-4}$ & 32.2 & 55.1 & 54.6 & 1.26 \\
C & 266 & 243 & 7.51 & 7.77 & 3.34 \\
D & 43.1 & 339 & 17.74 & 18.3 & 3.06 \\
\hline
\end{tabular}

Table 2.1: Rise of a 3D bubble of a light fluid 2 in a heavy fluid $1\left(\rho_{1}^{\prime} / \rho_{2}^{\prime}=1000\right.$; $\left.\mu_{1}^{\prime} / \mu_{2}^{\prime}=100\right)$. Comparison of terminal Reynolds number between experiments [22] and 3D-LBM numerical simulations.

The computed terminal shapes of the bubble for cases (A)-(D) are compared in Table 2.2 to experimental results [22] and to independent numerical results [24]. 


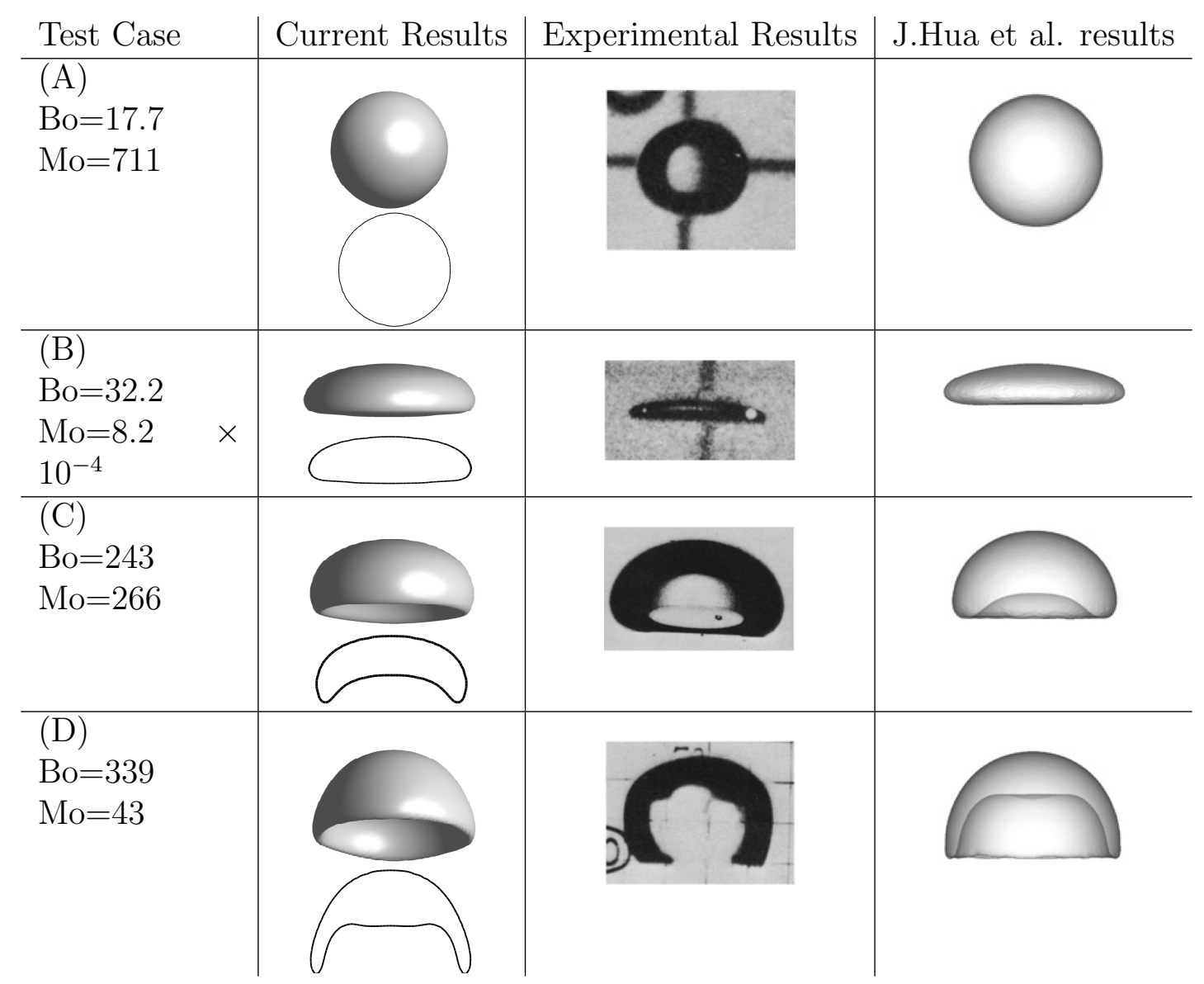

Table 2.2: Comparison of the computed terminal shapes of a rising bubble of fluid 2 into fluid 1, with the experimental results of [22] and the numerical simulations of Hua et al.[24], for $\rho_{1}^{\prime} / \rho_{2}^{\prime}=1000$ and $\mu_{1}^{\prime} / \mu_{2}^{\prime}=100$.

We see that our computed shapes agree very well with the reference data and that spherical, ellipsoidal, and spherical caps have been formed depending on the case.

Figure 2.8 further shows the computed time evolution of the bubble shape during its rise, for case $(\mathrm{C})$. During the early stages of the simulation, buoyancy forces are dominant and accelerate the bubble. As the bubble picks up speed, viscous drag gradually changes its shape, bending it downstream. Eventually, the terminal shape of the bubble is formed when buoyancy, surface tension, and viscous force contributions are balanced.

Finally, in Figure 2.9, the computed bubble rising velocity as a function of 
time is compared to the numerical results of Hua et al. [24]. The agreement between both sets of numerical results is very good.

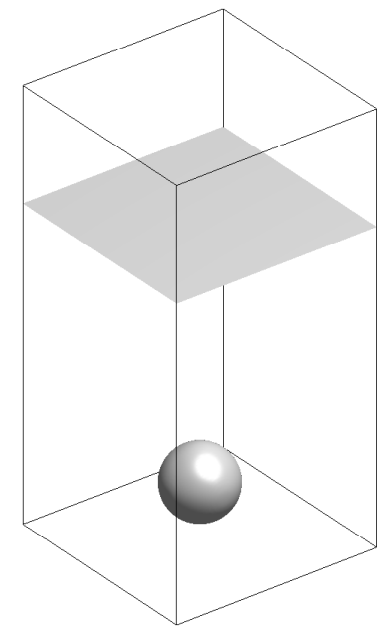

(a) $t^{*}=0$

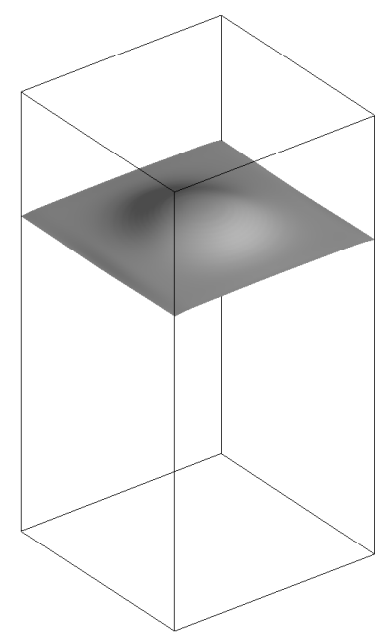

(d) $t^{*}=8$

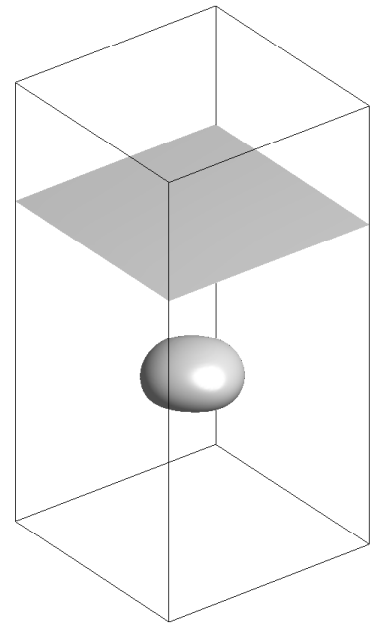

(b) $t^{*}=4$

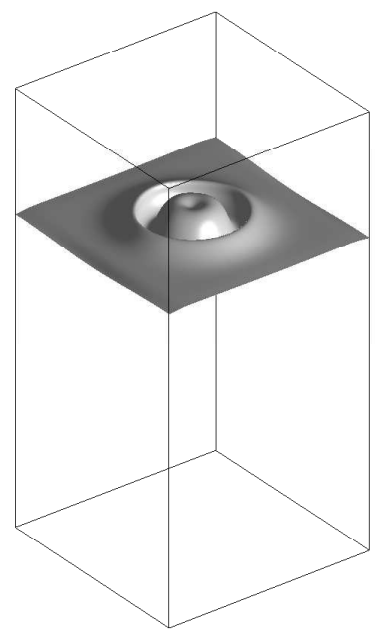

(e) $t^{*}=8.8$

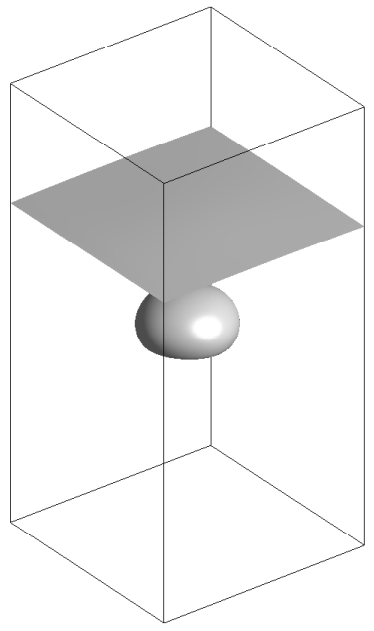

(c) $t^{*}=5$

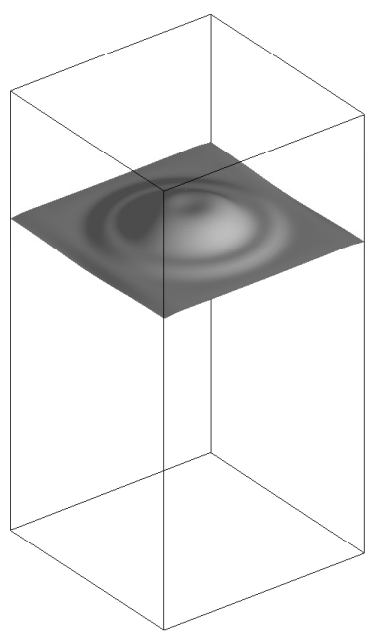

(f) $t^{*}=10.4$

Figure 2.8: Time evolution of a rising bubble of fluid 2 into fluid 1, for case (C), with $E t=243, M o=266, t^{*}=\sqrt{g / D} t, \rho_{1}^{\prime} / \rho_{2}^{\prime}=1000$, and $\mu_{1}^{\prime} / \mu_{2}^{\prime}=100$. 


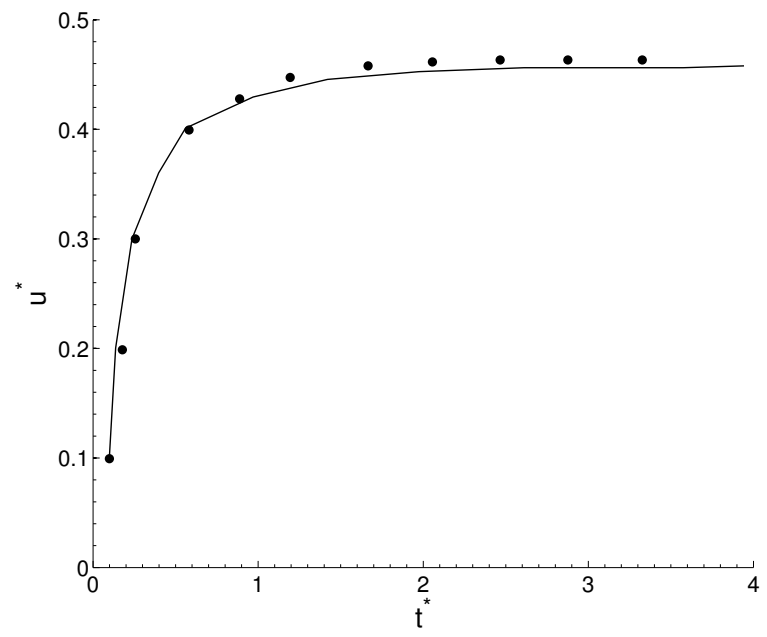

Figure 2.9: Rising bubble of fluid 2 into fluid 1: non-dimensional rising velocity $U^{*}=u / \sqrt{g D}$ as a function of non-dimensional time $t^{*}=\sqrt{g / D} t$ : 3D LBM results $(\cdot)$; experimental results of [24] (-) 


\subsubsection{Breaking wave}

In this last application, we solve the demanding test case of a 3D breaking ocean wave at an air-water interface. Earlier numerical simulations based on fully nonlinear potential flow theory [25, 26] showed that a periodic sinusoidal wave of large amplitude, with initial velocities specified from linear wave theory, is not stable in a fully nonlinear model and rapidly overturns and breaks, when used as an initial condition. This type of initialization has thus often been used to rapidly create a breaking wave, when validating both single or multiple fluid NS models [1]. To limit computational time, simulations typically assume spatial periodicity in the the main flow direction. Hence, this problem is still quasi-2D in the vertical plane, although 3D turbulent flow structures appear in the transverse direction to that of wave propagation.

The initial velocity field and interface shape for a 2D linear wave of height $H$ in depth $h$, specified in a 3D domain, are given by [1] (with $z$ denoting the vertical direction and $z=0$ at the undisturbed air-water interface),

$$
\begin{aligned}
& \eta=\frac{H}{2} \cos (k x) \\
& u=\frac{H}{2} \sigma \frac{\cosh k(h+z)}{\sinh \left(k h^{*}\right)} \cos (k x) \\
& w=\frac{H}{2} \sigma \frac{\sinh k(h+z)}{\sinh \left(k h^{*}\right)} \sin (k x) \\
& v=0
\end{aligned}
$$

where $\sigma=2 \pi / T$ is the wave angular frequency, $k=2 \pi / L$ is the wave number Figure 2.10, and $h^{*}=h+\epsilon$, where $\epsilon(y)=0.08 y$ is a small linear perturbation of the seafloor geometry, denotes a perturbed depth used in the model for triggering unsymmetrical flows and vortices in the transverse direction to the main flow, thus ensuring that turbulent structures will rapidly develop at high Reynolds numbers [1]. 
Because the finest LBM mesh resolution (i.e., largest grid) achievable on a single GPGPU in this application will not be fine enough to capture all the turbulent scales, as in [1], we use a Large Eddy Simulation (LES) model as a subgrid scale turbulence model. In LES, a spatial filter is applied to the velocity field, which should be fine enough that the largest turbulent structures of the flow are not filtered out [16]; in LBM-LES, $\Delta x$ is typically used as the filter length (see Krafczyk et al. 28] for details). In the Smagorinsky LES model, a turbulent eddy viscosity $\mu_{T}$ is added to the molecular viscosity $\mu$ for calculating total forces (viscous and turbulent sub-scale forces) in Eq. (2.9), which is defined as,

$$
\mu_{T}=\rho\left(C_{s} \Delta x\right)^{2}\|\boldsymbol{S}\|
$$

with $C_{s}$ the Smagorinsky constant and $\boldsymbol{S}$ the strain rate tensor being defined by,

$$
S_{\alpha \beta}=\frac{1}{2}\left(\frac{\partial u_{\alpha}}{\partial x_{\beta}}+\frac{\partial u_{\beta}}{\partial x_{\alpha}}\right)
$$

Since in our LBM scheme, spatial derivatives of the velocity field have already been calculated using Eq. (2.5), there is no need to recalculate them for computing the strain rate tensor, which makes using a LES quite efficient in our model.

The key dimensionless flow parameters for the periodic breaking wave problem are the Reynolds number, $R e=\rho_{w} c L / \mu_{w}$, the density ratio, $\rho_{w} / \rho_{a}$, and the viscosity ratio, $\mu_{w} / \mu_{a}$ (where $w$ and $a$ denote water and air respectively). Dimensionless wave parameters are the steepness $H / L$, which quantifies wave nonlinearity, and the relative depth, $h / L$, which quantifies dispersive effects. In the following application, the fluids characteristics are set to the properties of air and fresh water, i.e., $\mu_{w} / \mu_{a}=55, \rho_{w} / \rho_{a}=855$ with $\rho_{w}=1000 \mathrm{~kg} / \mathrm{m}^{3}$ and $\mu_{w}=10^{-3} \mathrm{Ns} / \mathrm{m}^{2}$.

Lubin et al. [1] solved this problem with a 3D-NS-VOF model, using a LES subgrid scheme, in a $250 \times 25 \times 75$ grid in the $x, y$, and $z$ directions $(z$ denoting the vertical direction), using a uniform grid size of $0.0004 \mathrm{~m}$. In the following we 
compare results of our 3D-LBM model to the latter study, while assessing their sensitivity to grid resolution. As in Lubin et al., we specify as initial condition a steep sinusoidal wave, with $H / L=0.13$ and $h / L=0.13$ (i.e., an intermediate depth wave). Based on linear wave theory, the wave celerity is $c=0.324 \mathrm{~m} / \mathrm{s}$ for a wave period of $T=0.308 \mathrm{~s}$ and a wavelength $L=0.1 \mathrm{~m}$, which results in a Reynolds number $R e=32,400$. Note that because of the short wavelength, surface tension effects are expected to play a significant role in the interface dynamics. In the LBM model, free slip boundary conditions are specified on the top and bottom boundaries of the computational domain, and periodic boundary conditions are applied on the four vertical sides (Figure 2.10).

We first solve the problem in a $256 \times 128 \times 64 \mathrm{LBM}$ grid (i.e., with $N=2.1$ million grid cells; referred to as "grid 1"), which has a resolution in the vertical plane similar to that of Lubin et al. and is wide enough in the lateral direction to ensure fully developed 3D turbulent flow structures. Then, to improve the resolution of breaker jets and better match Lubin et al.'s results in the vertical plane, we use a $512 \times 64 \times 128 \mathrm{LBM}$ grid (i.e., with $N=4.2$ million grid cells; referred to as "grid 2"). This larger grid, however, is narrower (one-eight of the length compared to half for grid 1) because of memory limitations on a single GPGPU (owing to the 3 sets of LBM distribution functions and related physical fields that need to be discretized), which will prevent fully turbulent structures from developing in the transverse direction. A convergence study of breaker shape to grid resolution, while maintaining a 4:1 ratio of horizontal to vertical grid size, finally shows that a 400x100 resolution in the vertical plane is sufficient to obtain converged breaker shapes. This allows increasing the width to a quarter the length, yielding 400x100x100 cells (4 million), for a grid referred to as grid 3 .

Figure 2.11 first shows the time evolution of the free surface profile, before 

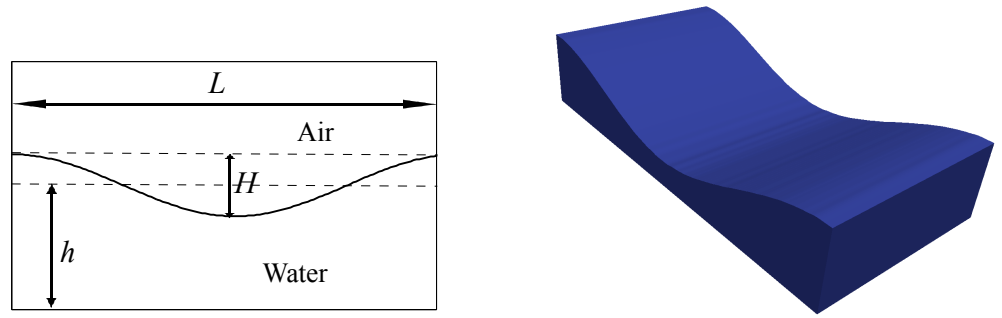

(a) $U t / D=0$

Figure 2.10: Definition sketch in the vertical plane $(x, z)$ for the initial interface profile of a large amplitude sinusoidal wave (left), used to initialize the 3D-LBM domain (right).

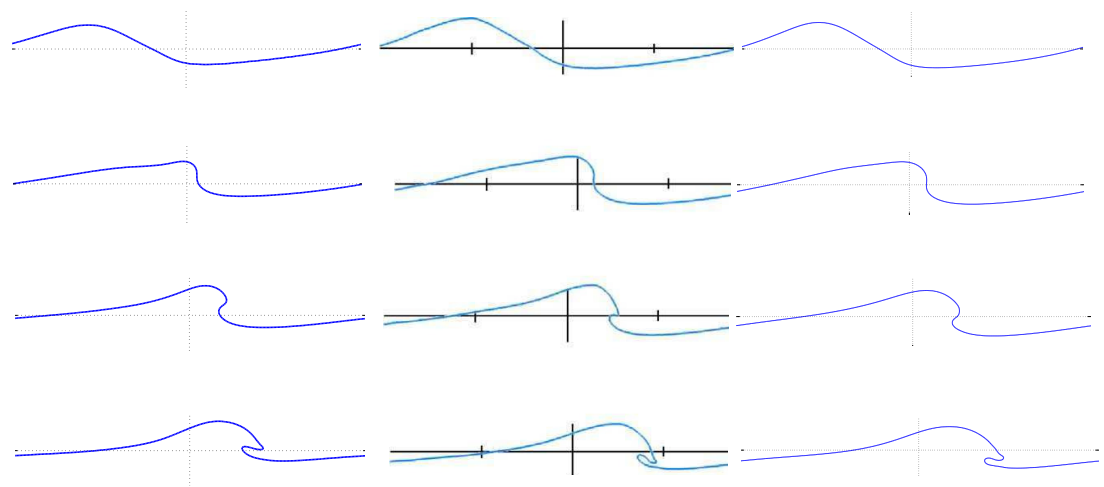

Figure 2.11: Time evolution of a 3D breaking sinusoidal wave with initial characteristics: $H / L=0.13$ and $h / L=0.13, R e=32,400$. Comparison of prebreaking surface profiles in the main vertical cross-section $(y=0$; Figure 2.10), at $t / T=0.17,0.3,0.38$, and 0.46 (top to bottom) in : (left, right) 3D-LBM results in grids 1 and 2, respectively; (middle) Lubin et al.'s 3D-NS-VOF results [1]. 
breaking occurs, computed in the middle lateral cross section ( $y=0$; Figure 2.10), at four times $t / T=0.17,0.3,0.38$ and 0.46 , in our 3D model for both grids 1 and 2, compared to Lubin et al.'s results [1]. As expected, the wave quickly overturns and develops a plunging jet. The agreement of our results with Lubin et al.'s results is good for both grids, although the shape of the plunging jet appears to be in better agreement in the finest resolution grid 2 .

To assess the convergence of LBM results with grid resolution, in Fig. 2.12, we compare results of LBM simulations up to impact of the breaker jet on the free surface, for 6 different grid resolutions, of which the first one is identical to grid 2 and the second one has the same resolution in the vertical plane as grid 3: (1) $512 \times 64 \times 128(N=4.2$ million); (2) $400 \times 50 \times 100(N=2$ million $) ;(3)$ $320 \times 40 \times 80(N=1$ million $) ;(4) 256 \times 32 \times 64(N=0.5$ million $) ;(5) 200 \times 25 \times 50$ $(N=0.25$ million $) ;$ and $(6) 128 \times 16 \times 32(N=0.066$ million $)$. All these grids have a mesh ratio of $8: 1: 2$, in order to study the convergence of the numerical scheme in similar conditions (i.e., their width is one-eigth their length and their height one quarter their length). Fig. 2.12 compares the computed air-water interface geometry, in the main-cross-section $(y=0)$ of the 3D-LBM domain, for clarity, only for four different resolutions (1, 2, 4 and 6). While large differences are observed between the last 3 coarsest resolutions 2, 4 and 6, these differences become quite small between the two finest resolutions 1 and 2 . Hence, both grid 2 and 3, seem to ensure convergence on the breaker shape in the vertical plane. To quantify convergence of the simulations, in the absence of detailed reference data, we compute the $L_{2}$ relative error norm of the air-water interface location between results in the 5 coarsest discretizations (2-6) and those in the finest one (1; grid 2). This error norm is plotted in Fig. 2.13) as a function of the resolution, expressed as the number of grid cells in the $x$ direction. Convergence clearly occurs and we 
see that it is approximately second-order in the log-log plot. [Note, however, while the proof of convergence is conclusive in such a grid-to-grid comparison, the rate of convergence is only indicative as one would have to use an independent converged solution as a reference to accurately calculate it.]

Fig (2.14) shows details of the splash-up generation after the plunging jet impacts the water surface. Our results for grid 1 and 2 are again compared with those reported by Lubin et al. [1], at 6 different times. [Note that no times were provided in Lubin et al.'s results, so we tried to find similar phases in our computations to perform this qualitative comparison.] In all sets of results, after the initial jet impact, a secondary rebound jet occurs while an air pocket is enclosed in the water. Later on, the rebound jet also impacts the free surface, enclosing an even larger air mattress in the process. While there are differences in the details of the computed free surface shapes, which can be attributed to the different free surface tracking/capturing methods, surface tension force computation, and discretization, overall, our results in both grids are in good qualitative agreement with those of Lubin et al. As for the pre-breaking results, however, we find that the agreement is much better for grid 2 results. The converged shape of the initial plunging jet was also much better modeled in this grid (Figure 2.12) and, as we see, this applies to the subsequent splash-up and flow structures in the vertical plane during post-breaking processes.

Finally, Figs. 2.15, 2.16, and 2.17 show snapshots of 3D-LBM results computed in grids 1,2 and 3, at eight different times, the first two being the same $(t / T=0.38$ and 0.46$)$ as for the overturning free surface profiles shown in Figure 2.11, while the last $\operatorname{six}(t / T=0.48$ to $t / T=1)$ illustrating the wave breaking and post-breaking decay processes up to one wave period, with the 3rd to 6th time levels matching some of the times shown in Figure 2.14. Overall, as expected from 


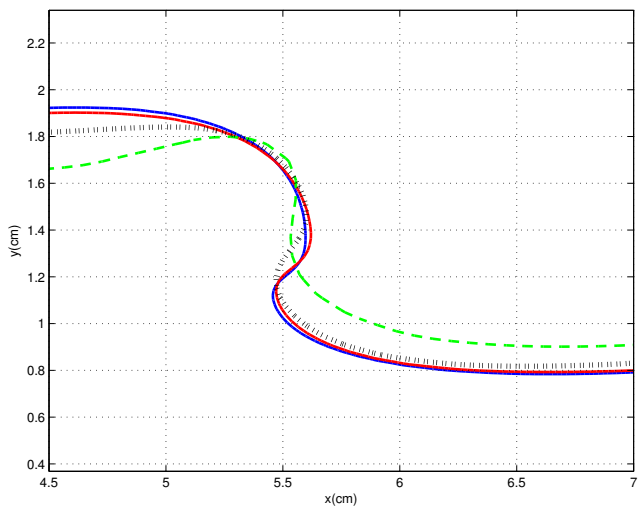

(a) $t=0.38 T$

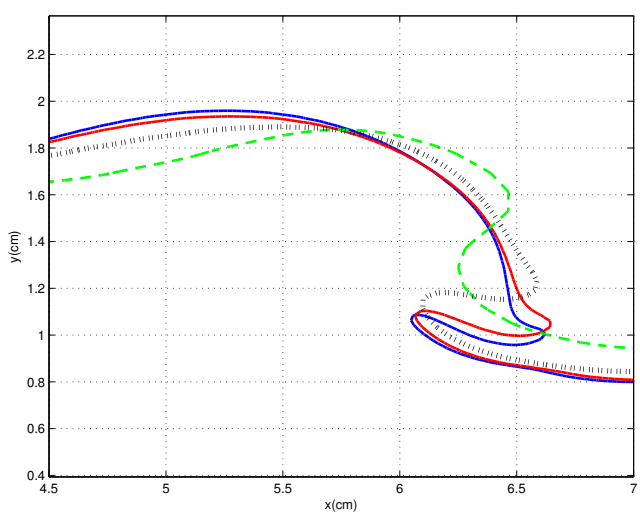

(b) $t=0.46 T$

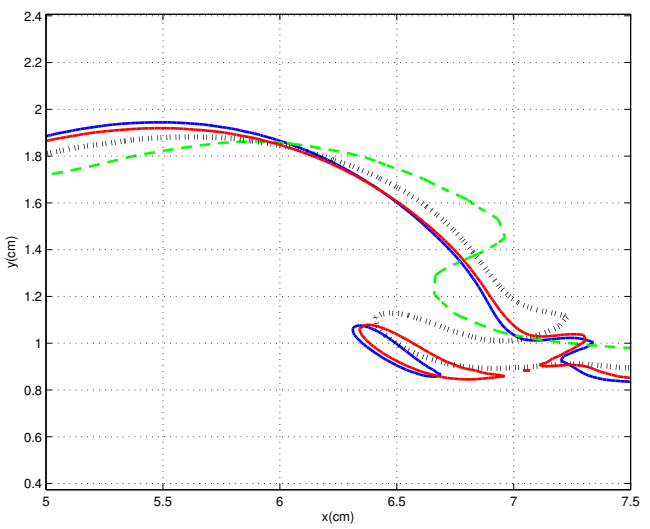

(c) $t=0.48 T$

Figure 2.12: Convergence study for the case of Fig. 2.11. Geometry of the airwater interface in the main cross-section $(y=0)$ for four different resolutions in a grid with dimension ratios 8:4:1 and number of cells in the $x$ direction: (1; blue; grid 2) 512; (2; red) 400; (4; black) 256; and (6; green) 128. 


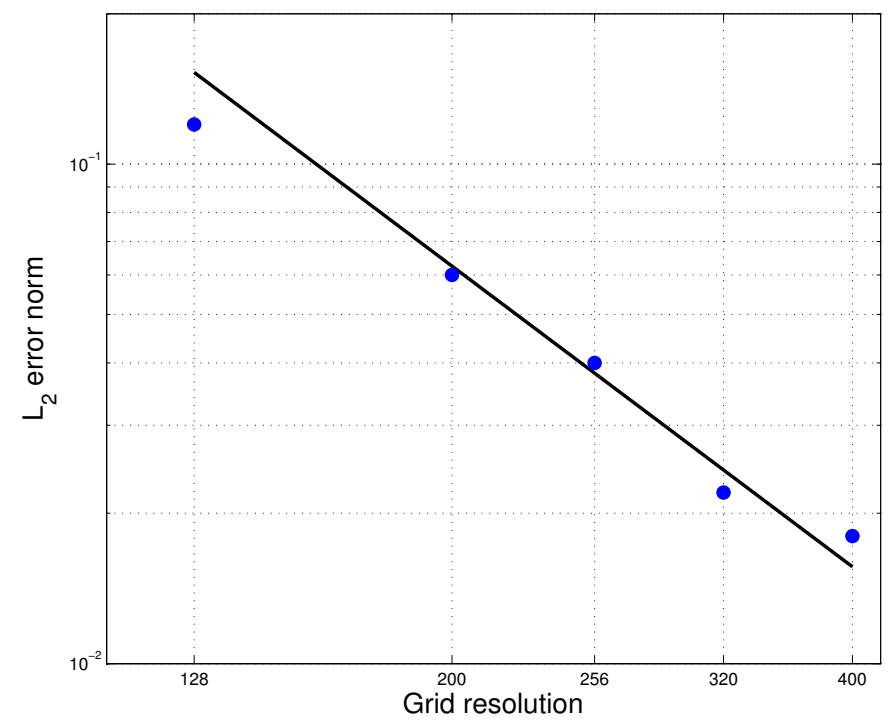

Figure 2.13: Case of Fig. 2.12, $L_{2}$ relative error norm, between surface profiles computed for resolution (1) and those for resolutions (2-6), as a function of resolution in the $x$ direction

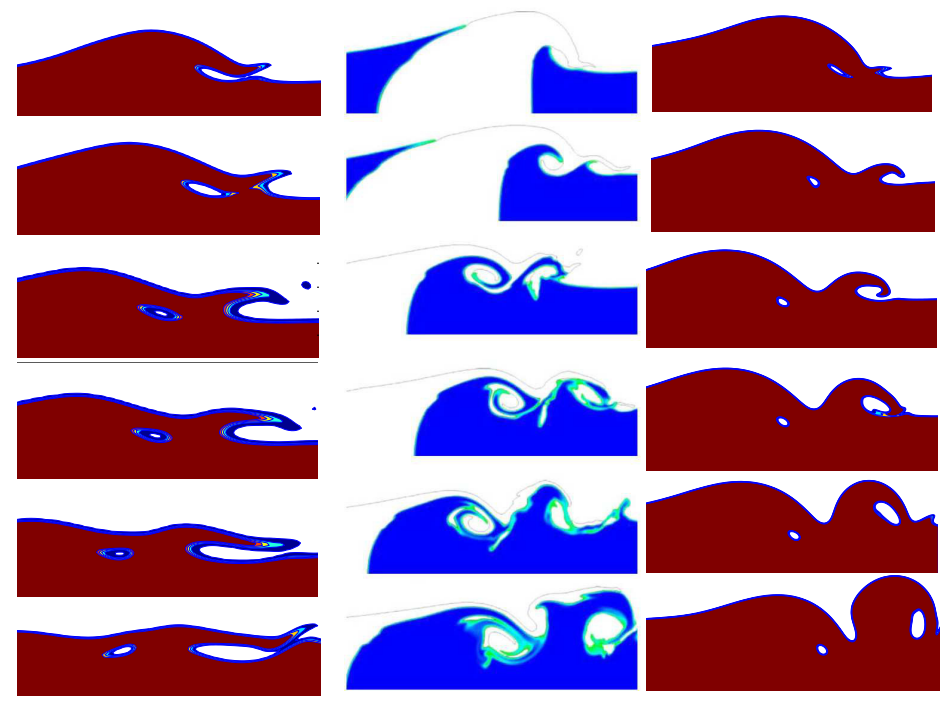

Figure 2.14: Case of Fig. 2.11. Time evolution of a 3D overturning breaking wave in the middle lateral cross section $(y=0$; Figure 2.10). Comparison of details of splash-up generation in the main vertical cross-section $(y=0$; Figure 2.10) at $t / T=0.48,0.5,0.52,0.56,0.64,0.68$ (top to bottom) in : (left, right) 3D-LBM results in grids 1 and 2, respectively; (middle) Lubin et al.'s 3D-NS-VOF results [1]. 


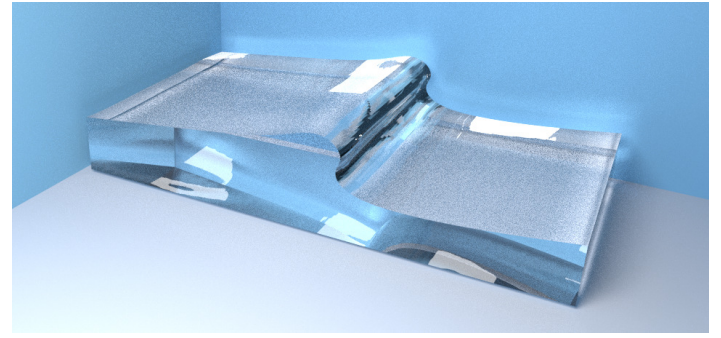

(a) $t / T=0.38$

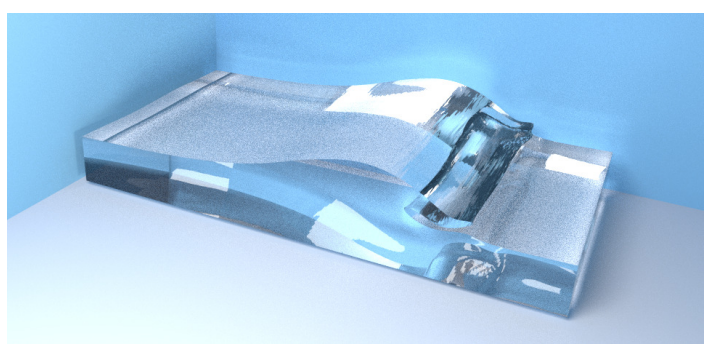

(c) $t / T=0.48$

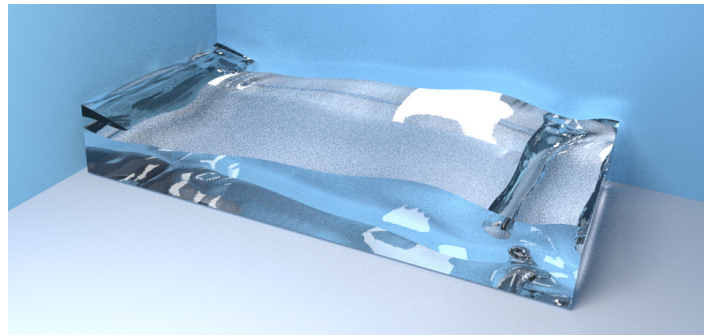

(e) $t / T=0.64$

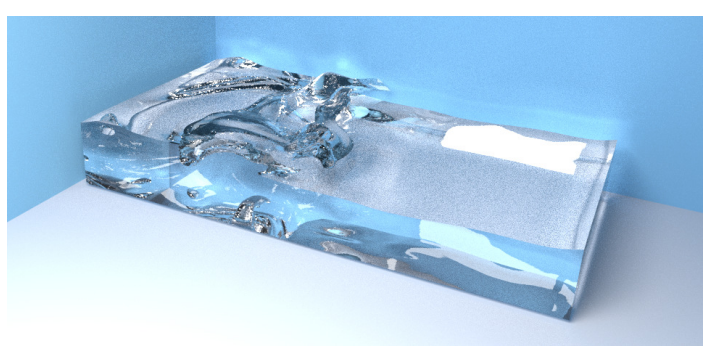

(g) $t / T=0.85$

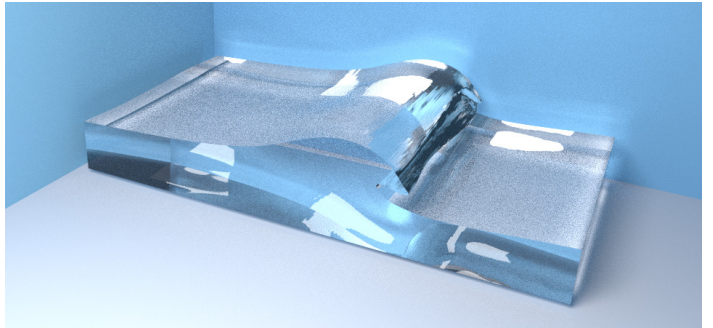

(b) $t / T=0.46$

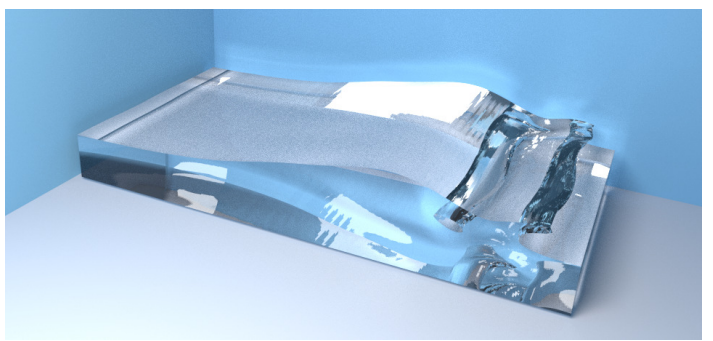

(d) $t / T=0.56$

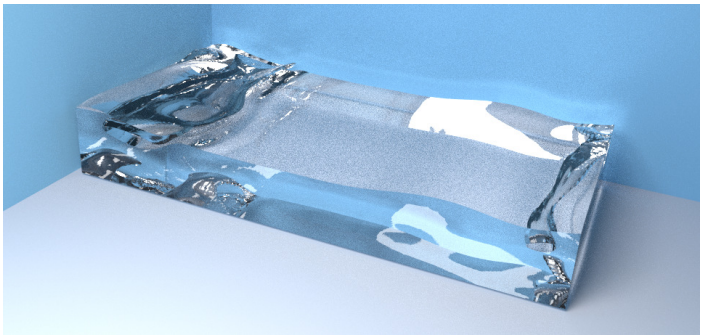

(f) $t / T=0.68$

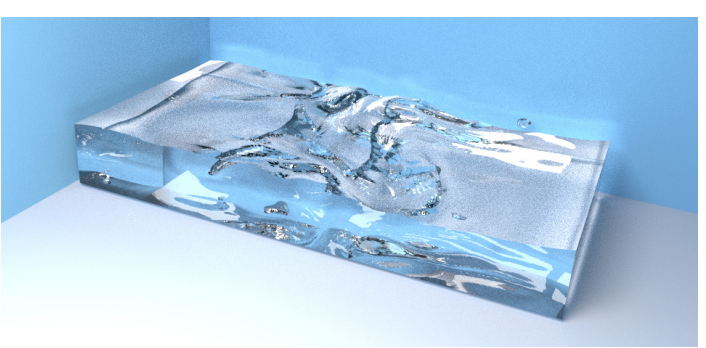

(h) $t / T=1$

Figure 2.15: Time evolution of a 3D periodic breaking wave for the same case as in Figure 2.11, for grid 1 with $256 \times 128 \times 64$ cells in the $x, y$ and $z$ directions. 


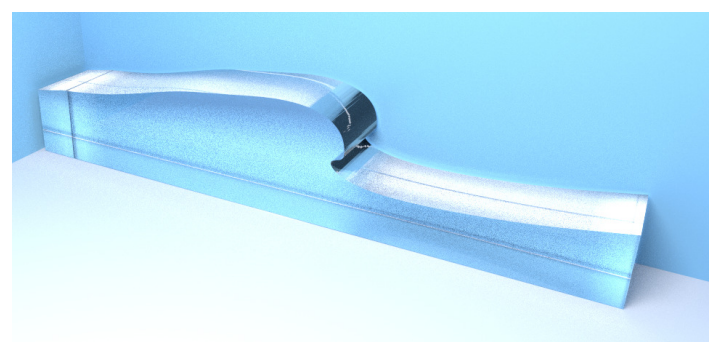

(a) $t / T=0.38$

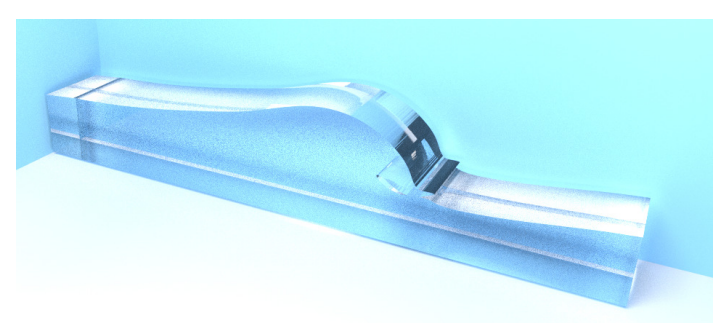

(c) $t / T=0.48$

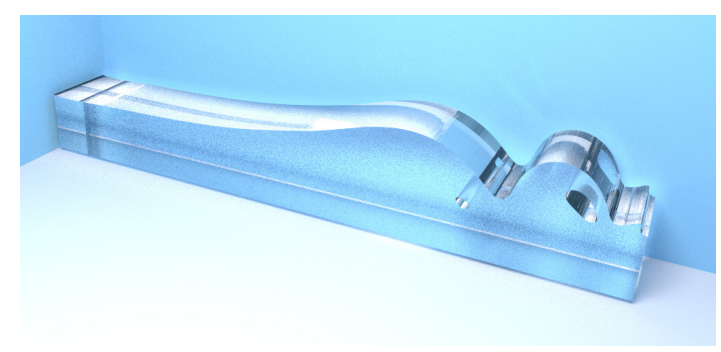

(e) $t / T=0.64$

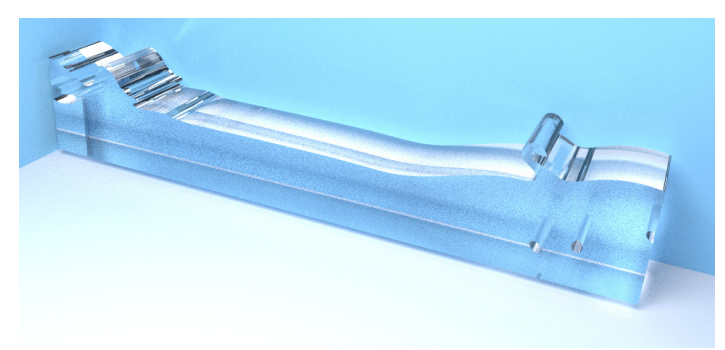

(g) $t / T=0.85$

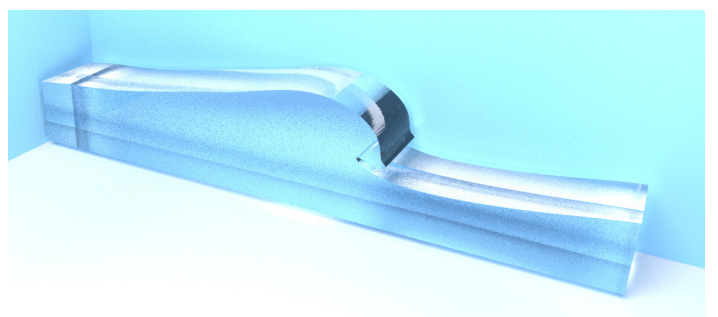

(b) $t / T=0.46$

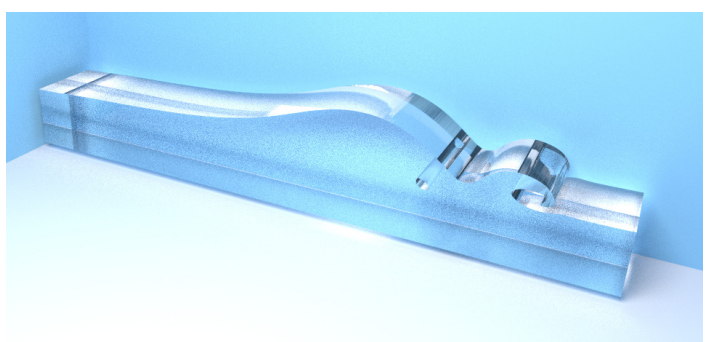

(d) $t / T=0.56$

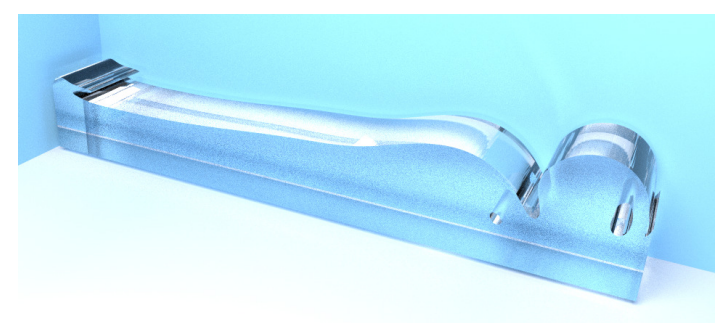

(f) $t / T=0.68$

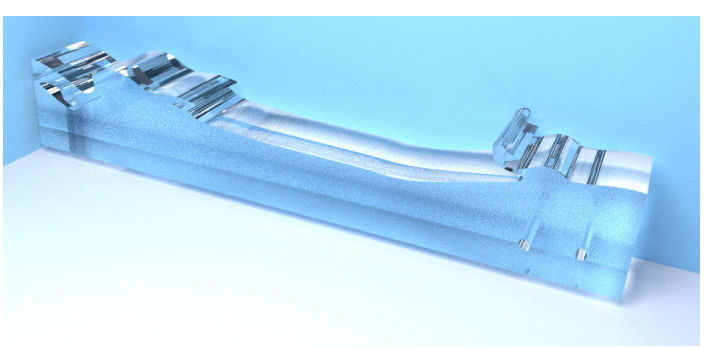

(h) $t / T=1$

Figure 2.16: Time evolution of a 3D periodic breaking wave for the same case as in Figure 2.11, for grid 2 with $512 \times 64 \times 128$, in the $x, y$ and $z$ directions. 


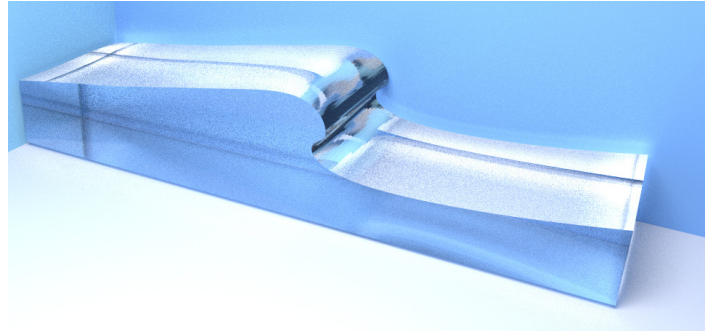

(a) $t / T=0.38$

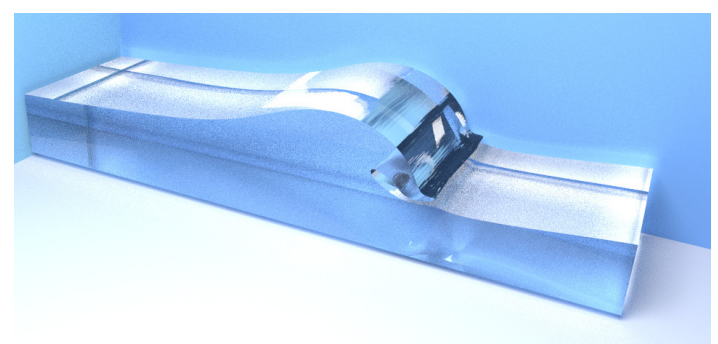

(c) $t / T=0.48$

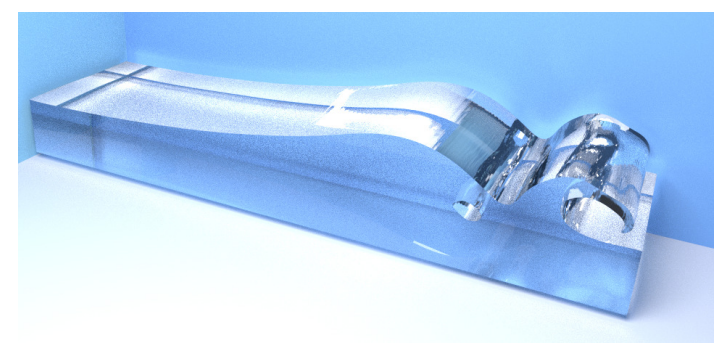

(e) $t / T=0.64$

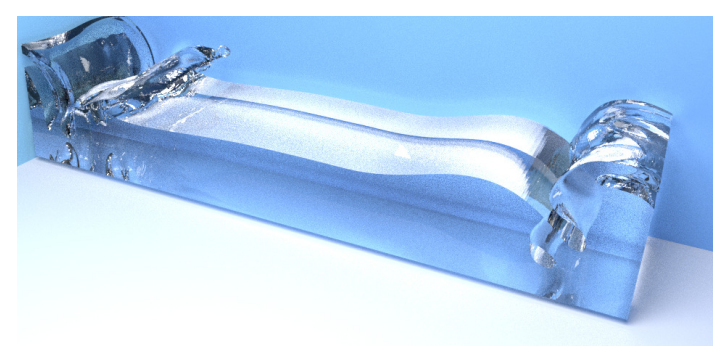

(g) $t / T=0.85$

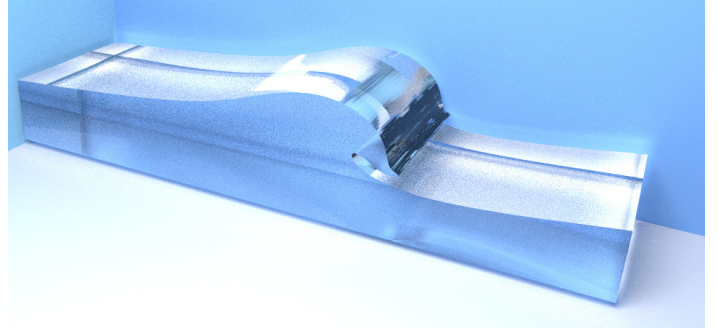

(b) $t / T=0.46$

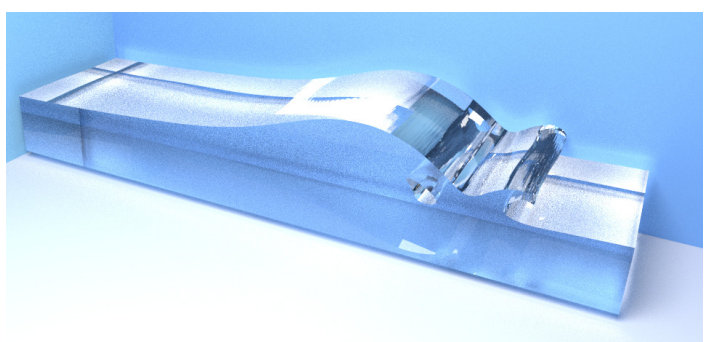

(d) $t / T=0.56$

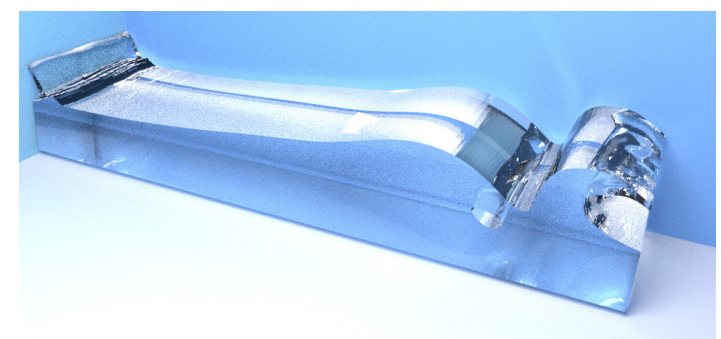

(f) $t / T=0.68$

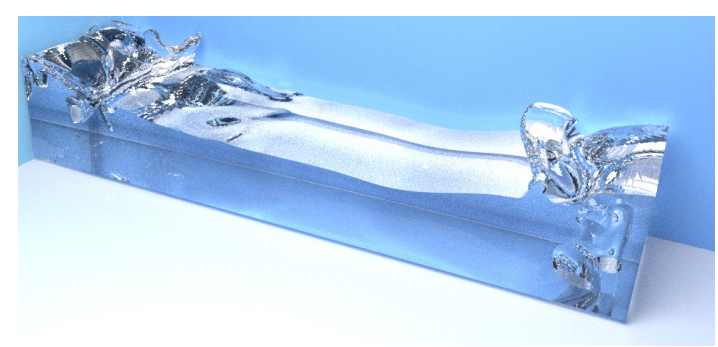

(h) $t / T=1$

Figure 2.17: Time evolution of a 3D periodic breaking wave for the same case as in Figure 2.11, for grid 3 with $400 \times 100 \times 100$, in the $x, y$ and $z$ directions. 
previous results in vertical cross-sections, both the wave and breaking processes are much better simulated (and resolved) in grids 2 and 3 than in grid 1 . However, as could also have been expected from the much larger width of grid 1 (four times that of grid 2 and twice that of grid 3), for the same length to height ratio 4:1 in all grids, the flow structure becomes much more 3D in grid 1, with larger turbulent structures appearing in the transverse direction towards the end of the simulations. While almost no turbulent structures appear in grid 2, however, grid 3 seems to offer a reasonable compromise, with a good much of breaker shapes in the vertical plane and some turbulent structures in the transverse direction.

More specifically, in the first two snapshots, while the wave is not broken yet and hence is still fairly 2D in all grids, one can already see in grid 1 the appearance of lateral flow perturbations triggered by the perturbation in seafloor bathymetry. Later on, at $t / T=0.48,0.56$ and 0.64 , shortly after the plunging jet has impacted the free surface, as in Figure 2.14, a sizable splash up jet is generated in all 3 grids, but much more so in grids 2 and 3, which creates a secondary rebound jet, with the enclosure by the plunging water jet of a cylindrical air cushion. At this stage, the flow is still fairly laterally uniform, although some transverse instabilities are clearly starting to grow, particularly in grid 1. Later on, in the last three snapshots, the rebound jet impacts the free surface and the wave gradually dissipates. In grids 1 and 3, the flow at this stage clearly becomes turbulent and 3D, with large lateral variations. We also see sizable pockets of air enclosed in the water at a few locations. In grid 2, however, lateral turbulence does not significantly develop and, likely as a result of this quieter dynamics, the wave dissipates more slowly than in grids 1 and 3. Clearly, one would need to use a finer lateral discretization in grid 2, to achieve a fully realistic simulation, with sufficient turbulence.

As indicated above, we find that grid 3 results offer a good comprise between 
the better resolved breakers of grid 2 and the more intense lateral turbulence

of grid 1. Nevertheless, all these simulations still lack the stronger splash up seen in many observations of breaking ocean waves, with many water parcels being ejected in the air and widespread air bubble generation in the water. The likeliest reason for this is that our 3D-LBM discretization is not fine enough to properly resolve such phenomena, due to the memory/grid size limitation when using a single GPGPU. This could only be improved with a significant increase in grid size, which would require implementing our numerical code on a large GPUGPU-CPU cluster, where one could perhaps achieve on the order of 100 million grid cells (i.e., an improvement in resolution of nearly a factor of 3 in each direction, as compared to grid 2). In view of the maximum of 4.2 million cells we were able to achieve here on a single GPGPU, this would require using on the order of 25 GPGPUs. As an indication, recent NS-VOF two-fluid flow simulations by Lubin and Glockner [29], implemented on thousands of CPUs, have shown that very fine flow structures can be resolved in breaking wave simulations, when using tens of millions of grid cells. This extension of our 3D-LBM model to an implementation on large GPGPU clusters, which is hardware already available, will be left for future work.

\subsection{Conclusions and Outlook}

In this paper, we presented the development, numerical implementation and validation of an efficient 3D Lattice Boltzmann model (LBM) for the simulation of multiple-fluid flows with high density ratios, at high Reynolds number. This model both extends our earlier 2D model [5] to 3D but, importantly, to high Reynolds number flows. While some of the principles for our method were first proposed by Inamuro et al. [9], we improved the method by using: (i) a 3D D3Q19 
lattice operator; (ii) a more accurate and rigorous formulation of body forces and surface tension terms; and (iii) a new interface capturing method based on solving a convection-diffusion Cahn-Hilliard equation. Additionally, our model relies entirely on the LBM, using three different sets of LBM particle distribution functions for solving (pressureless) Navier-Stokes (NS) equations, a Poisson equation for the pressure field in order to correct the velocity field to satisfy mass conservation, and the Cahn-Hilliard equation. This complete LBM approach makes our numerical scheme particularly well-suited, and thus efficient, for an implementation on heavily parallel GPGPU boards (NVIDIA TESLA 20170). Here, with an implementation in double precision on a single GPGPU, we could achieve up to $20 N U P S$ for discretizations of up to 4.2 million grid cells in the breaking wave application. The latter simulation was run in a 512x64x128 grid for one wave period, or 101136 time steps, yielding a total computational time of about 6 hours. By contrast, the simulation run in the 400x100x100 grid (4 million nodes) had a larger time step and only took $3.5 \mathrm{hrs}$ to compute one wave period.

To achieve higher NUPS values, the Poisson equation solution should be further accelerated, since it must be performed at each time step and represents a large fraction of the computing time. This could be achieved by using a multigridtype method, which would require smaller numbers of iterations to compute the velocity field correction terms.

The new 3D LBM model was applied to the simulation of various benchmark problems, for which numerical results were found to agree well with the reference data in most cases; convergence of numerical results with grid size was also assessed, for the demanding application of a breaking ocean wave. This latter case, however, revealed the limitations of the present single GPGPU implementation of the model, due to maximum grid size/resolution that can be achieved. This 
limitation could be overcome by implementing the model on multiple GPGPUs, by way of a parallel MPI algorithm. Except for a small overhead in computational time, due to communication and data exchange between various GPGPUs and the CPUs, one would expect solving problems in larger grids in nearly the same total time on a GPGPU cluster. For instance, the last application for a breaking wave could be solved in a grid 25 times larger (110 million nodes; improvement by a factor of nearly 3 in resolution) using 25 GPGPUs, perhaps in as little as 7 hrs of computations (assuming a 15\% overhead). This will be left for future work.

We will also note that the convergence and comparison study made for the 3D breaking wave application seemed to indicate that a finer discretization (perhaps by a factor of 1.5-2 in each direction) is required in the 3D-LBM, to produce results comparable to those of the 3D-NS-VOF model of Lubin et al. [1]. Indeed, in their work, they used a 250x25x75 grid while our grids 2 and 3 that matched their results in the vertical plane were 512x64x128 and 400x100x100, respectively. Such differences in grid size, however, are to be expected. While they directly discretize and solve the NS equations, we solve a set of discrete Boltzmann equations, whose solution converges to that of the NS equation. In doing so, we discretize the LBM distribution functions and not the physical fields (i.e., velocity and pressure). Nevertheless, we expect that the present efficient GPGPU implementation of our 3D-LBM model, should achieve much smaller computational times, even with larger discretizations, for similar accuracy.

On the basis of the present results, we are confident that the multi-GPGPU implementation in combination with an acceleration of the pressure Poisson equation, would make our numerical model a powerful tool for simulating complex 3D two-fluid flows, in order to explore new physics or solving complex engineering problems, with high fluid density ratios at a high Reynolds number. 


\section{Acknowledgment}

The authors wish to acknowledge support from grant OCE-09-27014 of the

US National Sciences Foundation (NSF) Physical Oceanography Program.

\section{List of References}

[1] D. Yu, R. Mei, L. Luo, W. Shyy, Viscous flow computations with the method of lattice Boltzmann equation, Prog. Aerospace Sc. 39 (2003) 329-367.

[2] J. Tölke, M. Krafczyk, Implementation of a Lattice Boltzmann kernel using the Compute Unified Device Architecture developed by nVIDIA, Comp. Visualization in Sc. 1 (2008) 29-39.

[3] J. Tölke, M. Krafczyk, Teraflop computing on a desktop PC with GPUs for 3D CFD, Intl. J. Comp. Fluid Dyn. 22 (2008) 443-456.

[4] C. Janßen, M. Krafczyk, Free surface flow simulations on GPGPUs using LBM, Computer and Mathematics. with Applications 61 (12) (2011) 35493563.

[5] A. Banari, C. Janßen, S. T. Grilli, M. Krafczyk, Efficient GPGPU implementation of a lattice Boltzmann model for multiphase flows with high density ratios, Computer and Fluids 93 (2014) 1-17.

[6] S. Chapman, T. Cowling, The mathematical theory of nonuniform gases, Cambridge University Press, 1970.

[7] C. Janßen, S.T. Grilli, M. Krafczyk, On enhanced non-linear free surface flow simulations with a hybrid LBM-VOF model, Comp. Math. with Applications, $65(2), 211-229$.

[8] T. Lee, C. Lin, A stable discretization of the lattice Boltzmann equation for simulation of incompressible two-phase flows at high density ratio, J. Comp. Phys 206 (2005) 16-47.

[9] T. Inamuro, T. Ogata, S. Tajima, N. Konishi, A lattice Boltzmann method for incompressible two-phase flows with large density differences, J. Comp. Phys. 198 (2004) 628-644.

[10] X. Shan, H. Chen, Lattice Boltzmann model for simulating flows with multiple phases and components, Phys. Rev E47 (1993) 1815-1819.

[11] T. Lee, Effects of incompressibility on the elimination of parasitic currents in lattice Boltzmann method for binary fluids, Comp. and Math. 58 (2009) 987-994. 
[12] H.W. Zheng, C. Shu, Y.T. Chew, A lattice Boltzmann model for multiphase flows with large density ratio, J. Comput. Phys 218 (2006) 353-371.

[13] X. He, S. Chen, R. Zhang, A lattice Boltzmann scheme for incompressible multiphase flow and its application in simulation of Rayleigh-Taylor instability, J. Comp. Phys. 152 (1999) 642-663.

[14] J. Cahn, J. Hilliard, Free energy of a nonuniform system, J. Chem. Phys 28 (1958) 258-267.

[15] Y. Qian, D. d'Humieres, P. Lallemand, Lattice BGK models for Navier-Stokes equation, Europhys. Lett. 17 (1992) 479-84.

[16] Z. Guo, C. Zhen, B. Shi, Discrete lattice effects on the forcing term in the lattice Boltzmann method, Phys. Rev. E 65 (4)(2002) 046308.1-046308.6 .

[17] M. Swift, W. Osborn, J. Yeomans, Lattice Boltzmann simulation of liquid-gas and binary fluid systems, Phys. Rev. E54 (1996) 5041-5052.

[18] A.M. Worthington, A Study of Splashes, London Longmans Green, 1908.

[19] D.A. Weiss, A.L. Yarin, Single drop impact on liquid film: Neck distortion, jetting, tiny bubble entrainment, and crown formation, J. Fluid. Mech. 385 (1999) 229.

[20] R. Rioboo, C. Bauthier, J. Conti, M. Voue, J. De Coninck, Experimental investigation of splash and crown formation during single drop impact on wetted surfaces, Exp. in Fluids 35 (2003) 648-652.

[21] R. Clift, J. Grace, M. Weber, Bubbles, drops, and particles, Academic Press. 1978.

[22] D. Bhaga, M. Weber, Bubbles in viscous liquids: shapes, wakes and velocities, J. Fluid Mech. 105 (1981) 61-85.

[23] L. Amaya-Bower, T. Lee, Single bubble rising dynamics for moderate Reynolds number using lattice Boltzmann method, Comp. and Fluids 39 (2010) 1191-1207.

[24] J. Hua, J.F. Stene, P. Lin, Numerical simulation of 3D bubbles rising in viscous liquids using a front tracking method, J. Comp. Phys. 227 (2008) 3358-3382.

[25] M.S. Longuet-Higgins, E.D. Cokelet, The deformation of steep surface waves on water: a numerical method of computation, Proc. Royal Soc. A350 (1976) $1-26$.

[26] S. T. Grilli, J. Skourup, I.A. Svendsen, An efficient boundary element method for nonlinear water waves, Engng. Anal. Boundary Elements 6 (1989) 97-107. 
[27] P. Lubin, S. Vincent, S. Abadie, J. Caltagirone, Three-dimensional large eddy simulation of air entrainment under plunging breaking waves, Coast. Engng. 53 (2006) 631-655.

[28] M. Krafczyk, J. Tölke, L.S. Luo, Large-eddy simulations with a multiplerelaxation-time LBE model, Intl. J. Modern Phys. B17(01-02) (2003) 33-39.

[29] P. Lubin, S. Glockner. Detailed numerical investigation of the threedimensional flow structures under breaking waves Proc. 7th Conf. on Coast. Dynam. (Arcachon, France) (2003) 1127-1136.

[30] X. He, L.S. Luo, Lattice Boltzmann model for the incompressible NavierStokes equation, J. Stat. Phys. 88 (1997) 927-944.

\section{List of References}

[1] P. Lubin, S. Vincent, S. Abadie, and J.-P. Caltagirone, "Three-dimensional large eddy simulation of air entrainment under plunging breaking waves," Coastal Engineering, vol. 53, pp. 631-655, 2006.

[2] D. Jacqmin, "Calculation of two-phase Navier-Stokes flows using phase-field modeling," Journal of Computational Physics, vol. 155, pp. 96-127, 1999.

[3] S. O. Unverdi and G. Tryggvason, "A front-tracking method for viscous, incompressible, multi-fluid flows," Journal of Computational Physics, vol. 100, pp. 25-37, 1992.

[4] J. Brackbill, D. B. Kothe, and C. Zemach, "A continuum method for modeling surface tension," Journal of Computational Physics, vol. 100, pp. 335-354, 1992.

[5] J. Cahn and J. Hilliard, "Free energy of a nonuniform system," Journal of Chemical Physics, vol. 28, pp. 258-267, 1958.

[6] M. Swift, W. Osborn, and J. Yeomans, "Lattice Boltzmann simulation of non-ideal fluids," Physical Review Letters, vol. 75, pp. 830-833, 1995.

[7] X. He, S. Chen, and R. Zhang, "A lattice Boltzmann scheme for incompressible multiphase flow and its application in simulation of Rayleigh-Taylor instability," Journal of Computational Physics, vol. 152, pp. 642-663, 1999.

[8] T. Lee and C. Lin, "A stable discretization of the lattice Boltzmann equation for simulation of incompressible two-phase flows at high density ratio," Journal of Computational Physics, vol. 206, pp. 16-47, 2005.

[9] T. Inamuro, T. Ogata, S. Tajima, and N. Konishi, "A lattice Boltzmann method for incompressible two-phase flows with large density differences," Journal of Computational Physics, vol. 198, pp. 628-644, 2004. 
[10] S. Chapman and T. Cowling, The mathematical theory of nonuniform gases. Cambridge University Press, 1970.

[11] J. Tölke and M. Krafczyk, "TeraFLOP computing on a desktop PC with GPUs for 3D CFD," International Journal of Computational Fluid Dynamics, vol. 22, pp. 443-456, 2008.

[12] D. Rothman and J. Keller, "Immiscible cellular-automaton fluids," Journal of Statistical Physics, vol. 52, pp. 1119-1124, 1988.

[13] X. Shan and H. Chen, "Lattice Boltzmann model for simulating flows with multiple phases and components," Physical Review, vol. E47, pp. 1815-1819, 1993.

[14] T. Inamuro, T. Ogata, and F. Ogino, "Numerical simulation of bubble flows by the lattice Boltzmann method," Future Generation Computer Systems, vol. 20, pp. 959-964, 2004.

[15] J. Tölke and M. Krafczyk, "Implementation of a lattice Boltzmann kernel using the compute unified device architecture developed by nVIDIA," Computing and Visualization in Science, vol. 1, pp. 29-39, 2008.

[16] C. Janßen and M. Krafczyk, "Free surface flow simulations on GPGPUs using LBM," Computers and Mathematics with Applications, vol. 61, no. 12, pp. 3549-3563, June 2011. 


\section{CHAPTER 3}

The simulation of turbulent particle-laden channel flows by the Lattice Boltzmann method

To be submitted with co-authors: Stephan T. Grilli, Tetsu Hara, Christian Janßen and Yackar Mauzole (alphabetical order). 


\begin{abstract}
We perform Direct Numerical Simulation (DNS) of three-dimensional (3D) turbulent flows in a rectangular channel, with a Lattice Boltzmann Method (LBM), efficiently implemented on heavily parallel General Purpose Graphical Processor Units (GPGPUs). The channel is made of two solid plates in between which the fluid flows under the effect of a pressure gradient; spatial periodicity conditions are specified in the two along-plate directions. After validating the method for a single fluid, for standard boundary layer problems, we study changes in mean and turbulent properties of particle-laden flows, as a function of particle size and concentration. The problem of physical interest for this application is the effect of water droplets on the turbulent properties of a high speed air flow near a solid surface. To do so, we use a Lagrangian tracking approach for a large number of rigid spherical point particles, whose motion is forced by drag forces caused by the fluid flow; particle effects on the latter are in turn represented by distributed volume forces in the LBM. Results suggest that, while mean flow properties are only slightly affected, unless a very large concentration of particles is used, the turbulent vortices present near the boundary are significantly damped and broken down by the turbulent motion of the heavy particles, and both turbulent Reynolds stresses and the production of turbulent kinetic energy are decreased due to the particle effects. We also find that the streamwise component of turbulent velocity fluctuations is increased, while the spanwise and wall-normal components are decreased, as compared to the single fluid channel case. Additionally, the streamwise velocity of the carrier (air) phase is slightly reduced in the logarithmic boundary layer near the solid walls.
\end{abstract}




\subsection{Introduction}

The study of turbulent particle-laden flows is important for various fluid mechanics problems of scientific and practical interest. Such flows indeed frequently occur in nature and in industrial applications, e.g., as a result of the release of sea sprays in the lower atmospheric turbulent boundary layer above the ocean, dust removal, pollutants in the atmosphere and the ocean. Although turbulent particle-laden flows have been extensively studied in past two decades, the full understanding of the modifications they induce to turbulent properties is still lacking. The complexity of such fluid flows and the numerous governing parameters that must be considered in their numerical simulations, for instance, particle size and mass fraction, Reynolds number, and particles collisions, make it a challenging problem of which many aspects are still poorly understood and thus actively researched.

The behavior of dispersed multiphase flows can be classified according to the level of interactions between the carrier (fluid) and the dispersed (particle) phase. The latter have been graphically illustrated by Elghobashi [1], in a diagram where the different interactions that can be observed in such flows are represented in terms of two parameters, the particle: (1) Stokes number St, which is the ratio of particle response time $\tau_{p}$, a measure of their inertia, normalized by the turbulence time scale $\tau_{e}$ or the Kolmogorov time scale $\tau_{K}=\sqrt{\nu / \varepsilon}$ (where $\nu$ is the fluid kinematic viscosity and $\varepsilon$ the rate of dissipation of turbulent kinetic energy). and (2) volume fraction $\Phi_{p v}$. In particular, Elghobashi indicates that, when $\Phi_{p v}<10^{-6}$, the flow governs the particle trajectories, but the dispersed phase has a negligible effect on flow turbulence: this is a case where the interactions between the two phases are a one-way coupling only (i.e., from fluid to particles). For intermediate volume fractions, ranging in $10^{-6} \leq \Phi_{p v} \leq 10^{-3}$, the momentum 
transfer from the particles to the flow turbulence is large enough to alter the turbulent structures [1]: this is a case where the interactions between the two phases are a two-way coupling (i.e., from fluid to particles, and from particles to fluid). In the case of very dense suspensions, when $\Phi_{p v}>10^{-3}$, strong interactions (i.e., collisions) between particles become important, and the interactions are called a four-way coupling (i.e., between carrier fluid and particles, and among particles). While one-way and two-way coupled systems have been extensively investigated, both numerically and experimentally [2], the four-way coupling implies that the dynamics of droplets, such as break-up or coalescence, should be taken into account, which represents a much larger computational challenge than simply considering rigid particles, which is adequate for the first two less dense cases. Hence, few studies have been devoted to this topic. Considering our targeted application to water sprays in airflow, for which the particle volume fraction will stay below the threshold for dense suspension, this latter case will be considered outside the scope of the present work, which will be devoted to the middle situation of two-way coupling.

In the following, we discuss some of the key findings of earlier work performed for moderately dense suspensions. Rashidi et al. [3] experimentally studied wall turbulence with polystyrene particle of various sizes. Results showed that the larger particles enhance Reynolds shear stresses and also increase the number of wall ejections, while the smaller particles lead to opposite effects on the turbulent fields. Squires and Eaton [4], in their two-way coupled direct numerical simulations of isotropic turbulence, observed that heavy particles collect preferentially in regions of low vorticity and high strain rate. Pan et al. [5] numerically investigated effects of near-neutral density solid particles on a turbulent channel flow. They reported 
that particles smaller than the dissipative length scale reduce the turbulence intensity and Reynolds stresses, whereas particles that are somewhat larger increase intensity and stresses. Li et al. [6] numerically studied the behavior of particleladen gases in a small Reynolds number vertical downward channel flow. Effects of particle-particle collisions are considered in their work. They reported that particle feedback forces cause the turbulent structures to become more anisotropic as the particle concentration is increased. For small mass fractions, the particles cause an increase in the gas flow rate. Also, particles tend to increase the characteristic length scale of the fluctuations in the streamwise components of velocity.

Most recently, Richter and Sullivan [7, 8] performed a series of direct numerical simulations to highlight the role of sea sprays in the momentum transfer between the atmosphere and the ocean. Sea spray droplets were modeled as heavy inertial particles suspended in a turbulent airflow, and droplets were represented by solid pointwise particles, whose individual trajectory was tracked over time in a Lagrangian way. They reported that, for typical diameters of sea spray droplets (typically between $10 \mu \mathrm{m}$ and $1 \mathrm{~mm}$ ), mechanical effects dominate over the thermal effects. By studying the momentum budget, they introduced a "spray" stress, which compensates for the decrease in Reynolds stresses by providing a feedback effect on the turbulence. They showed that the drag coefficient based on the total stress remains almost unchanged in the presence of sea sprays, while the drag coefficient based on the turbulent stress is reduced. Zhao et al. [9] also performed two-way coupled direct numerical simulations of turbulent channel flows, with a Lagrangian point particle approach. They pointed out that for sufficiently large particle response times (a measure of the particle inertia that will be defined later), the Reynolds shear stresses and the turbulence intensity in the spanwise and wallnormal directions were attenuated, whereas velocity fluctuations were increased in 
the streamwise direction.

Here, similar to some of these recent studies, we perform direct numerical simulations to study in detail the impact of point particles on properties of turbulent channel flows, in the particle mass fraction range that is relevant to the water (sea) spray particles in a high velocity gas (air) flow. To do so, we implement and use a three-dimensional Lattice Boltzmann Method (LBM) to solve Navier-Stokes (NS) equations and use a two-way coupling approach, between particles and the fluid carrier phase, in which the particle feedback forces are expressed as distributed volume forces in the LBM. In particular, the effects of particle volume fraction and size are studied on a series of mean and turbulent channel flow properties.

This article is organized as follows: the LBM used for solving the fluid flow in the carrier phase is first described in section 3.2. The Lagrangian particle tracking method is then explained in section 3.3. Results for a series of relevant simulations are presented next in section 3.4 and conclusions and a discussion of the main results are finally given in section 3.5.

\subsection{Direct Numerical Simulation of fluid flows with a Lattice Boltz- mann Method}

A three-dimensional (3D) model based on the Lattice Boltzmann Method (LBM) is used in this study for performing Direct Numerical Simulations (DNS) of the fluid flow for the carrier phase in particle-laden flows. The LBM is used here, because 3D-DNS on a large grid are computationally demanding problems that require using High a Performance Computer (HPC) platform and for such problems the LBM can be very efficiently implemented on heavily parallel General Purpose Graphical Processing Units (GPGPUs) [10, 13]. Instead of solving the macroscopic NS momentum equations, LBM models solve discretized mesoscopic Boltzmann equations, which are collision-propagation equations, for fluid particles 
moving on a regular lattice. It can be shown that for sufficiently small values of space and time steps, Mach (Ma) and Knudsen (Kn) numbers, the LBM solution converges to that of the NS equations. The Boltzmann equation thus governs particle distribution functions, $f(\boldsymbol{x}, t, \boldsymbol{\xi})$, which specify the probability of finding a fluid particle at position, $\boldsymbol{x}$ at time $t$, with particle velocity $\boldsymbol{\xi}[11,12$. The fundamental Boltzmann equation reads,

$$
\frac{\partial f(\boldsymbol{x}, t, \boldsymbol{\xi})}{\partial t}+\boldsymbol{\xi} \cdot \frac{\partial f(\boldsymbol{x}, t, \boldsymbol{\xi})}{\partial \boldsymbol{x}}+\boldsymbol{B} \cdot \frac{\partial f(\boldsymbol{x}, t, \boldsymbol{\xi})}{\partial \boldsymbol{\xi}}=\Omega
$$

where $\boldsymbol{B}$ denotes the external body forces. The left hand side of the Boltzmann equation is of advection type, while the right hand side contains the collision operator $\Omega$, which describes the interaction of particles.

\subsubsection{LBM governing equations}

For flows in a continuum, with a low Knudsen number, discretized velocities $\boldsymbol{e}_{i}$ are introduced to yield a model of reduced computational cost. In this discretized formulation, a particle is only allowed to move from a given grid (lattice) point, in a limited number of directions and for specific distances. With these assumptions, Eq 3.1 transforms into a set of discrete Boltzmann equations,

$$
\frac{\partial f_{i}(\boldsymbol{x}, t)}{\partial t}+\boldsymbol{e}_{i} \cdot \frac{\partial f_{i}(\boldsymbol{x}, t)}{\partial \boldsymbol{x}}+\boldsymbol{B} \cdot \frac{\partial f_{i}(\boldsymbol{x}, t)}{\partial \boldsymbol{e}_{i}}=\Omega_{i}
$$

In this work the so-called D3Q19 regular sub-lattice model is used for the discretization of the velocity space around one grid point (Fig. 3.1). The discrete velocities $e_{i}$ for this model are $(i=0, \ldots, 18)[13$,

$$
\boldsymbol{e}_{i}=c .\left\{\begin{array}{r|rrrrrr|rrrrrrrrrrrr}
0 & 1 & -1 & 0 & 0 & 0 & 0 & 1 & -1 & 1 & -1 & 1 & -1 & 1 & -1 & 0 & 0 & 0 & 0 \\
0 & 0 & 0 & 1 & -1 & 0 & 0 & 1 & -1 & -1 & 1 & 0 & 0 & 0 & 0 & 1 & -1 & 1 & -1 \\
0 & 0 & 0 & 0 & 0 & 1 & -1 & 0 & 0 & 0 & 0 & 1 & -1 & -1 & 1 & 1 & -1 & -1 & 1
\end{array}\right\}
$$




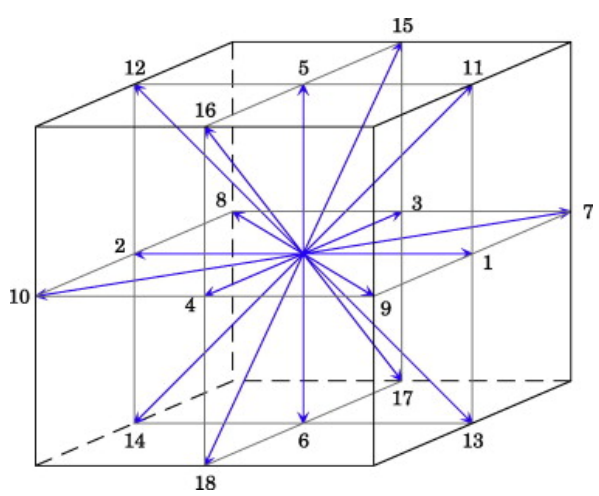

Figure 3.1: D3Q19 sub-lattice model used in the present LBM, where the blue vectors indicate the 18 possible vectors of motion (both direction and length) for a particle to move on the lattice according to Eq. (3.2).

where $c=\Delta x / \Delta t$ is the lattice speed, with $\Delta x$ the regular lattice space step and $\Delta t$ the time step, which usually takes a value of unity. This means that a particle with velocity $c$ will travel one lattice grid cell over one time step.

A finite difference discretization in space and time over a grid cell yields the Lattice Boltzmann (LBM) equation,

$$
f_{i}\left(\boldsymbol{x}+\boldsymbol{e}_{i} \Delta t, t+\Delta t\right)=f_{i}(\boldsymbol{x}, t)+\Delta t \Omega_{i}+\Delta t B_{i}
$$

where $B_{i}$ represents the discretized effects of body forces $B_{\alpha}$ in Eqs. 3.23 .22 . Following Buick and Greated [14] the latter can be expressed as $F_{i}=w_{i} \rho_{0} e_{i j} B_{j} / c_{s}^{2}$, where $c_{s}=c / \sqrt{3}$, is the speed of sound, and $w_{i}$ is lattice weight factor, which for the D3Q19 lattice is,

$$
w_{0}=\frac{1}{3} ; w_{1, . .6}=\frac{1}{18} ; w_{7, \ldots, 18}=\frac{1}{36}
$$

Eq. 3.4 may be split up into a collision step, during which the particle distribution functions change from equilibrium state due to the collision of the particles, and a propagation step, where the evolved particle distribution functions are moved to the respective neighboring grid points.

$$
\bar{f}_{i}(\boldsymbol{x}, t)=f_{i}(\boldsymbol{x}, t)+\Omega_{i}+\Delta t B_{i} \quad \text { Collision step }
$$




$$
f_{i}\left(\boldsymbol{x}+\boldsymbol{e}_{i} \Delta t, t+\Delta t\right)=\bar{f}_{i}(\boldsymbol{x}, t) \quad \text { Propagation step }
$$

For the collision operator, a so-called multiple relaxation time (MRT) has been used instead of the more widely used single relaxation time (SRT) collision operator, which is not stable for high Reynolds number flows [12]. In the MRT model, the particle distribution functions are transformed into moment space before relaxation. The moments $m=M f$ are labeled as,

$$
m=\left(\rho, e, \epsilon, j_{x}, q_{x}, j_{y}, q_{y}, j_{z}, q_{z}, 3 p_{x x}, 3 \pi_{x x}, p_{w w}, \pi_{w w}, p_{x y}, p_{y z}, p_{x z}, m_{x}, m_{y}, m_{z}\right)
$$

and denote the mass density $m_{0}=\rho$, the part of the kinetic energy independent of density $\left(m_{1}=e\right)$, the part of kinetic energy square independent of density and kinetic energy $\left(m_{2}=\epsilon\right)$, and the momentum $\left(m_{3,5,7}=j_{x, y, z}\right) ; m_{4,6,8}=q_{x, y, z}$ are related to heat fluxes, $m_{9,11,13,14,15}$ are related to the symmetric traceless viscous stress tensor, $m_{16,17,18}$ are third-order moments and $m_{10,12}$ are two fourth-order moments [15, 16].

The collision operator for MRT reads,

$$
\Omega_{i}=\boldsymbol{M}^{-1} \boldsymbol{S}\left(\boldsymbol{M f}-m_{i}^{e q}\right)
$$

$M$ is the transformation matrix from distribution functions to moment space $(m=$ $M f$ and $f=M^{-1} m$ ) and is given in [16]; $m_{i}^{e q}$ are the equilibrium moments given 
by,

$$
\begin{array}{lr}
m_{0}^{e q}=\rho, & m_{1}^{e q}=e^{e q}=\rho_{0}\left(u_{x}^{2}+u_{y}^{2}+u_{z}^{2}\right) \\
m_{3}^{e q}=j_{x}^{e q}=\rho_{0} u_{x}, & m_{5}^{e q}=j_{y}^{e q}=\rho_{0} u_{y} \\
m_{7}^{e q}=j_{z}^{e q}=\rho_{0} u_{z}, & m_{9}^{e q}=3 p_{x x}^{e q}=\rho_{0}\left(2 u_{x}^{2}-u_{y}^{2}-u_{z}^{2}\right) \\
m_{11}^{e q}=p_{z z}^{e q}=\rho_{0}\left(u_{y}^{2}-u_{z}^{2}\right), & m_{13}^{e q}=p_{x y}^{e q}=\rho_{0} u_{x} u_{y} \\
m_{14}^{e q}=p_{y z}^{e q}=\rho_{0} u_{y} u_{z}, & m_{15}^{e q}=p_{x z}^{e q}=\rho_{0} u_{x} u_{z} \\
m_{2,4,6,8,10,12,16,17,18}^{e q}=0 &
\end{array}
$$

where $\rho_{0}$ is a constant reference density and $\rho$ is the variable density. The velocities are derived from the represented momenta: $u_{\alpha}=j_{\alpha} / \rho_{0} . \quad S$ is a diagonal matrix given by,

$$
\boldsymbol{S}=\operatorname{diag}\left(0, s_{a}, s_{b}, 0, s_{c}, 0, s_{c}, 0, s_{c}, s_{d}, s_{e}, s_{d}, s_{e}, s_{d}, s_{d}, s_{d}, s_{f}, s_{f}, s_{f}\right)
$$

with $s_{d}=-\Delta t / \tau$, where $\tau$ is a relaxation time computed as a function of the fluid viscosity and the time step as,

$$
\tau=3 \frac{\nu}{c}+\frac{1}{2} \Delta t
$$

Parameters $s_{a}, s_{b}, s_{c}, s_{e}, s_{d}, s_{f}$ can be chosen freely in the range of [-20]. For the D3Q19 MRT model, d'Humieres et al. [16] set these relaxation rates for optimal stability to,

$$
s_{a}=-1.19 s_{b}=-1.4 s_{c}=-1.2 s_{e}=-1.4 s_{f}=-1.98 \text {. }
$$

These values will be used here.

Finally, both the velocity and pressure fields are obtained from their respective momenta as,

$$
u_{\alpha}=\frac{j_{\alpha}}{\rho_{0}}
$$




$$
p=\rho c_{s}^{2}
$$

It can been shown with a Champan-Enskog expansion that the LBM solution converges to that of the incompressible Navier-Stokes equation up to some orders of small parameters,

$$
\begin{gathered}
\frac{\partial \rho}{\partial t}+\rho \frac{\partial u_{j}}{\partial x_{j}}=0+\mathcal{O}\left(\Delta t^{2}\right)+\mathcal{O}\left(\mathbf{M a}^{2}\right) \\
\rho\left\{\frac{\partial u_{j}}{\partial t}+u_{k} \frac{\partial u_{j}}{\partial x_{k}}\right\}=\frac{\partial \sigma_{j k}}{\partial x_{k}}+\rho B_{j}+\mathcal{O}\left(\Delta t^{2}\right)+\mathcal{O}\left(\mathbf{M a}^{2}\right)+\mathcal{O}\left(\mathbf{K n}^{2}\right)
\end{gathered}
$$

\subsubsection{Boundary condition on solid walls}

To simulate the no-slip conditions at a solid boundaries in the LBM, we specify a so-called "bounce-back" boundary condition, which requires the fluid velocity to be zero at stationary walls. Bounce back on link is used in this work, which gives a second-order of accuracy in space for straight walls [17]. In this scheme, the wall is placed half way between two lattice nodes and the calculation is only performed for nodes inside the domain. After the propagation step, the unknown particle distribution functions are set equal to the opposite post collision distribution functions at the same node (see Fig. 3.2,

$$
f_{i}\left(x_{b}, t+\Delta t\right)=\bar{f}_{\bar{i}}\left(x_{b}, t\right)
$$

\subsubsection{Definition of parameters for DNS of turbulent channel flows}

The computational domain used in the 3D-LBM model for the DNS of turbulent channel flows is sketched in Fig 3.3. Although no gravity will be used in the present applications, the effect of gravity will be simulated in future work No-slip boundary conditions are specified along the lower and upper wall boundaries in the $z$ direction. These boundaries represent two solid walls separated by a distance 


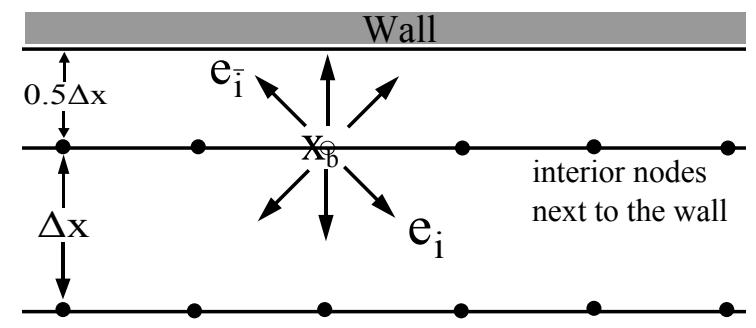

Figure 3.2: No-slip boundary condition at solid walls: sketch of LBM bounce back on link scheme.

$L_{z}=2 \delta$. Periodic boundary conditions are specified in both the streamwise $(x)$ and spanwise $(y)$ directions, which expresses that turbulence is homogeneous in these directions.

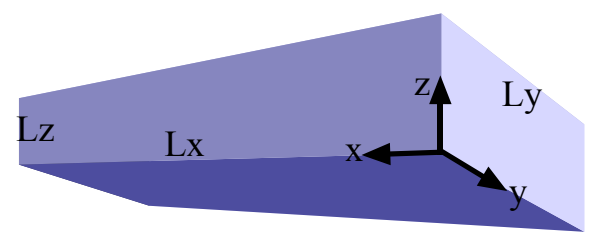

Figure 3.3: Sketch of computational domain for 3D-DNS LBM modeling of turbulent channel flows.

Next, we specify the grid resolution, in the form of the space step $\Delta x$, as a function of the desired Reynolds number. Near the wall, two important standard parameters are defined as the friction velocity,

$$
u_{\tau}=\sqrt{\frac{\tau_{w}}{\rho_{0}}}
$$

where $\tau_{w}$ is wall shear stress, and the viscous length scale,

$$
\delta_{\nu}=\nu \sqrt{\frac{\rho_{0}}{\tau_{w}}}=\frac{\nu}{u_{\tau}}
$$

The friction velocity can be used to define the friction Reynolds number [18],

$$
\boldsymbol{R e}_{\tau}=\frac{u_{\tau} \delta}{\nu}
$$

where $\delta$, as defined above, is half of channel width. Here, to compare with earlier seminal work on DNS of single fluid channel flows, we use $\mathbf{R e}_{\tau}=180$ in all the 
applications. Additionally, we found that this value was nearly the lowest possible Reynolds number that can be used to achieve turbulent conditions, which will allow using the largest possible domain on a given GPGPU.

According to Kim et al. [19, one of the requirements to achieve turbulent DNS is that the grid resolution be fine enough to resolve the smallest length turbulent scales. In particular, grid size should be on the order of the Kolmogorov length scale, which is estimated to be twice the viscous length scale, $\eta_{K} \simeq 2 \delta_{\nu}$; one also defines $\eta_{K}^{+}=\eta_{K} / \delta_{\nu} \simeq 2$ (where the superscript + denotes a quantity normalized by the viscous layer scale). In turbulent channel flows, the grid resolution should be at least equal to the normalized Kolmogorov length scale in the vertical (wall normal) direction. We therefore impose the normalized grid resolution to be $\Delta z^{+}=\eta_{K}^{+}=2$ in our configuration. The grid resolution in the other directions does not need to be as small as the Kolmogorov length scale. However, LBM models normally use regular lattices, where time, space and velocities are uniformly discretized in all directions, i.e., $\Delta x^{+}=\Delta y^{+}=\Delta z^{+}$. As a result, in the present case, the flow is over-resolved in the horizontal directions, while being appropriately resolved in the wall-normal direction [20]. This situation can be improved upon by using a varying grid resolution in different directions, as will be attempted in future work.

It should be pointed out that in the LBM, the length, time, and mass scales are usually defined in non-dimensional lattice units. Here, the symbols $L, T$ and $M$ are defined to characterize the length, time and mass scales, respectively, used in the lattice units. In particular, the grid spacing $\Delta x=1 L$ is set to unity in lattice length scale and time step $\Delta t=1 T$ to unity in lattice time scale. Thus, lattice speed $c=\Delta x / \Delta t$ is equal to $1 L / T$ in lattice unit. Though the LBM is for incompressible flows (Ma value lower than 0.3) the Mach number must be specified as a parameter. The Mach number of the present simulations will be based on the 
mean centerline velocity in the channel flow, which is the maximum mean velocity in the simulation. The speed of sound is $c_{s}=c / \sqrt{3}=0.577 L / T$ in lattice unit. Hence, to yield a low mean Mach number $\mathbf{M a}=0.2$, the mean centerline velocity was set to $U_{0}=0.115 L / T$ in lattice unit. The standard theoretical velocity profile for a wall boundary layer can be expressed as [18],

$$
U_{0}^{+}=\frac{1}{\kappa} \ln \left(\mathbf{R e}_{\tau}\right)+A
$$

with $A=5.5$ and $\kappa=0.4$, which can be used to express the relationship between centerline and friction velocity as, $U_{0}^{+}=U_{0} / u_{\tau}$.

In the applications, given the Mach and friction Reynolds number values, we get the mean velocity $U_{0}$, and Eq. 3.27 can be used to calculate the friction velocity. For $\mathbf{M a}=0.2$ and $\mathbf{R e}_{\tau}=180$, the friction velocity obtained from this equation is, $u_{\tau}=0.00622 L / T$ in lattice unit. With $\Delta x=1 L$, and the minimum requirement $\Delta x^{+}=2$, the viscous length scale is set to, $\delta_{\nu}=0.5 L$. Then, the kinematic viscosity of the simulation can be computed from the definition of the viscous length scale (Eq. 3.25); here, we find, $\nu=0.00311 L^{2} / T$, which based on Eq. 3.17) yields the relaxation time in LBM simulations, $\tau=0.5093 T$.

Based on the friction Reynolds number definition Eq. (3.26), we find the half width between walls to be $\delta=90 \mathrm{~L}$. Hence, the total number of lattice nodes in the wall normal direction $z$ is $2 \delta / \Delta x=180$ (Fig. 3.4). Note, from the implementation of the bounce back on link boundary condition, the first nodes next to the wall are placed at $0.5 \Delta x\left(\Delta x^{+}=1\right)$ away from the wall. Due to the limitation of using a single GPGPU memory (here 6Gb) in the numerical solution, the maximum lattice grid size that could be achieved was, $260 \times 260 \times 180$ (12.2 million nodes), in the $x, y$ and $z$ directions, respectively, which corresponds to a domain size of $(2.9 \delta, 2.9 \delta, 2 \delta)$

The mean wall shear stress can be computed from the definition of the friction 


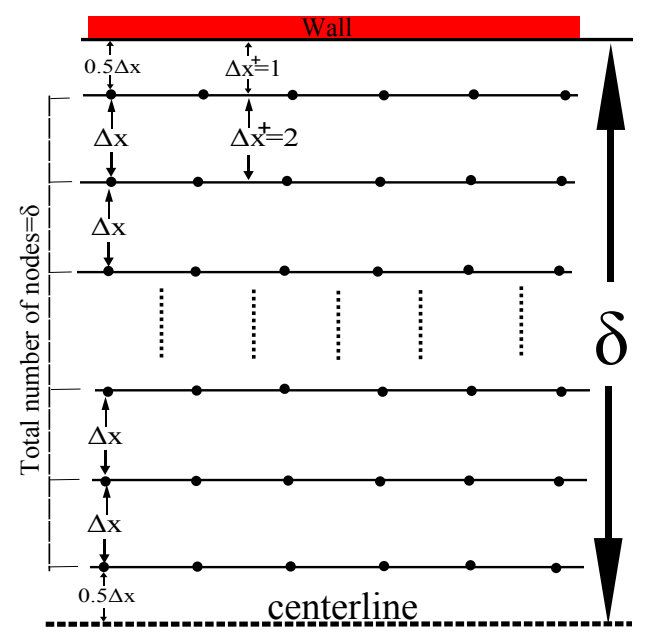

Figure 3.4: Sketch of half the computational domain in the wall-normal direction $z$, using the bounce back on link boundary condition. Total number of nodes for the half channel is $\delta / \Delta x=\delta$

velocity in Eq. (3.24), where the fluid reference density in lattice unit is $\rho_{0}=$ $1 M L^{-3}$. For the selected parameters, we find, $\tau_{w}=3.87 \times 10^{-5} M L^{-1} T^{-2}$. Finally, the mean pressure gradient required to force the fluid flow balances this wall shear stress integrated over the wall, and can thus be computed simply from a force balance (considering there are two walls),

$$
\tau_{w}=-\delta \frac{\partial p}{\partial x}
$$

This yields a pressure gradient, $\partial p / \partial x=4.30 \times 10^{-7} M L^{-2} T^{-2}$. In the LBM, this pressure gradient is actually implemented as a body force (as part of the $B_{i}$ term), as if the mean flow was forced by the $x$ component of gravity, $-\partial p / \partial x=\rho g_{x}$.

To summarize, the main parameters used for this DNS of turbulent channel flows in the 3D-LBM model, without particles, are (in lattice units):

- the viscous length scale $\delta_{v}=0.5 L$,

- the friction velocity $u_{\tau}=6.22 \times 10^{-3} L . T^{-1}$, 
- the kinematic viscosity $\nu=3.11 \times 10^{-3} L^{2} . T^{-1}$,

- the friction Reynolds number $\mathbf{R e}_{\tau}=180$.

\subsection{Modeling of particle-laden flows}

\subsubsection{Key physical concepts}

Here we perform the DNS modeling of turbulent channel flows, for a homogeneous fluid of density $\rho_{f}$, kinematic viscosity $\nu$, and velocity $u_{j}$ (using index notation; $j=1,2,3)$, in the presence of a dispersed phase made of a large number of small spherical particles, of density $\rho_{p}$ and velocity $v_{j}$ (here we assume $\rho_{p} \gg \rho_{f}$ ). Such flows were described by Maxey and Riley [23], who introduced the following assumptions that will also be made in our study:

- Particles are spherical with diameter $d_{p}$ and volume $V_{p}=\pi d_{p}^{3} / 6$, i.e., for simplicity, no shape effects are considered.

- Particles are smaller than the Kolmogorov length scale, i.e., $d_{p} \ll \eta_{K}$.

- Particles are rigid, i.e., no deformation is allowed and their diameter remains constant.

- The particle Reynolds number is small: $\mathbf{R e}_{p}=\frac{d_{p}\left|u_{j}-v_{j}\right|}{\nu}<1$, so that Stokes law of drag applies to compute their drag force.

Here, because the ratio of densities between the (water) "particles" and the fluid (air) is large, the particles are referred to as, heavy. For such cases, as a first-order, the force $F_{p j}$ exerted by the fluid on the particles, and vice versa, will be assumed to result only from the drag contribution. Hence, added-mass and lift effects are neglected. In this case, the equation of motion of each particle of mass $m_{p}=\rho_{p} V_{p}$, in the presence of a gravitational acceleration $g_{j}$ reads:

$$
m_{p} \frac{\mathrm{d} v_{j}}{\mathrm{~d} t}=\left(\rho_{p}-\rho_{f}\right) V_{p} g_{j}+F_{p j}
$$


The time derivative in this equation is a material derivative, following the particle motion. The external forces applied on each particle, in the right hand side, are weight minus buoyancy and drag forces. The latter is based on the instantaneous relative velocity of the flow at each particle location, and reads,

$$
F_{p j}=\frac{1}{2} C_{D} A_{p} \rho_{f}\left(u_{j}-v_{j}\right)\left|u_{j}-v_{j}\right|
$$

where $A_{p}=\pi d_{p}^{2} / 4$ is the particle cross-section and $C_{D}\left(\mathbf{R e}_{p}\right)$ is the drag coefficient. Using Stokes law of drag (i.e., a laminar flow search each particle), we have,

$$
C_{D}=\frac{24}{\mathbf{R e}_{p}}
$$

Combining Eqs. (3.30) and (stokes) we find the drag force applied by the fluid on each spherical particle located at $x_{p j}$,

$$
F_{p j}=3 \pi d_{p} \nu \rho_{f}\left(u_{j}-v_{j}\right)
$$

which, by reaction, is also the force applied to the fluid by each particle at the same location.

In the absence of gravity, which is an assumption also made in this work, the drag force exactly balances the particle inertia force and Eq. (3.29) yields,

$$
\frac{\mathrm{d} v_{j}}{\mathrm{~d} t}=\frac{F_{p j}}{m_{p}}=a_{j}
$$

with $a_{j}$ the particle acceleration. Combining Eqs. 3.30 and (3.33), the particle equation of motion reads,

$$
\frac{\mathrm{d} v_{j}}{\mathrm{~d} t}=\frac{\left(u_{j}-v_{j}\right)}{\tau_{p}} \quad \text { with } \quad \tau_{p}=\frac{\rho_{p} d_{p}^{2}}{18 \rho_{f} \nu}
$$

where $\tau_{p}$ is a new time scale referred to as particle response time.

The particle response time is the key characteristic time scale of multiphase flows and physically represents the time necessary for the dispersed phase to respond to fluctuations in flow velocity [1]. The larger $\tau_{p}$, the more inertia the 
particles will display. To the limit, when $\tau_{p}$ is very large, the particle inertia is so large that they behave like ballistic projectiles with their trajectory being nearly unaffected by the fluid flow. By contrast, when $\tau_{p}$ is close to zero, particles act as passive tracers for the flow, because they have so little inertia that they are able to freely follow the fluid motion, everywhere in the domain. In the present context, we will see that the magnitude of $\tau_{p}$ is a crucial factor for determining the behavior of the dispersed phase, by comparing it to a typical time scale of the turbulent flow.

In the following, we further discuss the key dimensionless parameters that govern fluid flows with a dispersed phase made of point particles, i.e., the : (1) particle Stokes number St; (2) particle volume fraction $\Phi_{p v} ;(3)$ fluid-particle density ratio $\rho_{f} / \rho_{p}$; and (4) particle Reynolds number $\mathbf{R} \mathbf{e}_{p}$. The first two parameters are the two most important ones identified by Elghobashi [1], and the fourth one was already discussed above.

(1) Particle Stokes number This parameter, defined as the ratio of the particle response time to the characteristic time scale of the turbulent flow, expresses the interactions between the turbulence structures and the dispersed phase. Several definitions of the Stokes number can be found in the literature, for DNS on wallbounded turbulence, which usually differ in the selected time scale of the flow. Indeed, the Kolmogorov (turbulent) time scale $\tau_{K}$ and the viscous time scale $\tau_{\nu}=$ $\delta_{\nu} / u_{\tau}$ can be used to define two different Stokes numbers,

$$
\mathbf{S t}_{K}=\frac{\tau_{p}}{\tau_{K}} \quad \text { and } \quad \mathbf{S t}^{+}=\frac{\tau_{p}}{\tau_{\nu}}=\frac{\tau_{p} u_{\tau}^{2}}{\nu}
$$

A review of the computational fluid dynamics literature indicates that, in the past 30 years, $\mathbf{S t}_{K}$ has been more widely used to characterize interactions between particle motions and flow structures, mainly because it applies to both isotropic and 
wall-bounded flows, making it a more "universal" parameter. However some recent studies have questioned its relevance for channel flows, in which the dissipation rate is not constant in the wall-normal direction, since $\varepsilon$ is larger near the walls. This implies a smaller Kolmogorov time scale near the walls, for a constant fluid viscosity, than at mid-channel width. Howewer, because it is hard to accurately quantify the variation of the dissipation rate across the channel width, most studies have used the time scale calculated at mid-channel width and assumed that it remains unchanged across the channel. By contrast, the viscous time scale does not present this problem as it does not depend on the distance from the wall, since the friction velocity is calculated from the wall stress and the fluid density. Hence, for wall-bounded turbulent flows, it appears more relevant to compare the particle response time to the viscous time scale, rather than to the Kolmogorov time scale. For instance, Richter and Sullivan [7, 8] and Zaho et al. [9] used $\mathbf{S t}^{+}$in their recent studies.

Particle volume fraction The volume fraction, $\Phi_{p v}$, quantifies how much of the dispersed phase is present in the flow. It is defined as the ratio of the volume occupied by the particles to the total volume (fluid plus particles),

$$
\Phi_{p v}=\frac{N_{p} \pi d_{p}^{3} / 6}{V_{f}+N_{p} \pi d_{p}^{3} / 6} .
$$

where $N_{p}$ denotes the number of particles and $V_{f}$ the fluid volume. Alternatively, the particle mass fraction can be used to specify the global concentration of particles in the flow. In a similar way to the volume fraction, it is defined as the ratio of the mass of all the dispersed particles $M_{p}=N_{p} m_{p}$ to the total mass of the system $M_{t}$

$$
\Phi_{p m}=\frac{N_{p}\left(\rho_{p} \pi d_{p}^{3} / 6\right)}{\left(\rho_{f} V_{f}+N_{p}\left(\rho_{p} \pi d_{p}{ }^{3} / 6\right)\right)}=\frac{N_{p} D_{\rho}}{\left(\frac{6 V_{f}}{\pi d_{p}{ }^{3}}+N_{p} D_{\rho}\right)}
$$


where $D_{\rho}$ is the the density ratio discussed below.

As discussed in the introduction, our work focuses on cases with intermediate

volume fractions, $10^{-6} \leq \Phi_{p v} \leq 10^{-3}$ corresponding to a two-way coupling between fluid and particle motions. This is the relevant regime to describe interactions occurring between the lower atmospheric turbulence and sea spray droplets at the ocean surface (Elghobashi [1]). In this regime, the particle suspension is not so diluted as to prevent particles from affecting flow turbulence or so concentrated that particles would strongly interact with each other (e.g., collisions, coalescence, break up).

Density ratio This is the ratio of the dispersed phase (particles) density over the the carrier phase (fluid) density (using Eq. (3.34),

$$
D_{\rho}=\frac{\rho_{p}}{\rho_{f}}=\frac{18 \nu}{d_{p}^{2}} \tau_{p} .
$$

When considering the forces applied by the carrier fluid onto the dispersed phase, the density ratio helps sorting out forces that can be neglected form those that are relevant to the problem (Maxey and Riley [23]). For instance, as seen in Eq. 3.38, the particle inertia characterized by the particle response time, becomes predominant when $D_{\rho}$ is very large. In our targeted application, the density ratio is on the order of 1000 , which means that particles can be qualified as heavy. In applications, the value of $D_{\rho}$ will remain unchanged unless specified otherwise.

\subsubsection{Numerical algorithm for particle tracking and volume forces}

This algorithm solves the particle equation of motion (3.34) by first-order finite difference (owing to the very small time steps), to compute the velocity $v_{j}$ of individual particles, as a function of time,

$$
v_{j}^{t+\Delta t}=v_{j}^{t}+\frac{\Delta t}{\tau_{p}}\left(u_{j}^{t}-v_{j}^{t}\right)+\mathcal{O}\left(\Delta t^{2}\right)
$$


The new position of each particle is then obtained via a second-order Taylor series expansion,

$$
x_{j p}^{t+\Delta t}=x_{j p}^{t}+\Delta t v_{j}^{t+\Delta t}+\frac{1}{2} \Delta t^{2} a_{j}^{t}+\mathcal{O}\left(\Delta t^{3}\right)
$$

where the particle velocity is obtained from Eq. 3.39 and the acceleration is obtained from Eq. (3.34).

As this is a Lagrangian time integration following each particle trajectory, the flow velocity $u_{j}$ must be computed at each particle instantaneous location. In the LBM model, however, velocities are only available at lattice nodes, which requires interpolating the velocity field. To do so, we use the accurate and efficient tricubic interpolation scheme of Lekien and Marsden [24. Once identified the grid cell in which the particle resides, the method interpolates velocities from neighboring lattice nodes. Because this is a regular lattice, a single set of coefficients $(64 \times 64$ matrix) is computed and repeatedly used in the interpolations, which saves both time and computational resources. In applications, to reduce computational time, particles are released in the fluid flow, once the latter is fully established (i.e., with proper turbulent structures). In this initial stage, particles are uniformly distributed and given the flow velocity at their release location.

The two-way coupling between the fluid and particle phases is operated in the LBM model by way of the volume force term $B_{j}$ in Eq. 3.22 . For a given lattice cell of volume $V_{c}$ containing $M_{p}$ particles, the total volume force per unit mass exerted in the $x_{j}$ direction is expressed as the opposite of the sum of all the drag forces applied to the particles, as,

$$
B_{j}=-\frac{1}{\rho V_{c}} \sum_{p=1}^{M_{p}} F_{p j},
$$

with $F_{p j}$ the drag force given by Eq. 3.32 for a single particle. In the LBM model, the contribution $B_{j}$ to the body force is uniformly distributed to the lattice 
nodes that are the vertices of the considered cell.

To summarize, in this particle-tracking/volume force approach, five steps are taken at each time step:

- the tricubic interpolation of the fluid velocity $u_{j}$ to find its value at the particle positions $x_{j p}$,

- the computation of the particle velocities $v_{j}$ from the fluid velocity field using Eq. (3.39),

- the time integration to find the new position of each particle using Eq. (3.40,

- the computation of the forcing terms $F_{p j}$ using Eq. 3.32 and contribution $B_{j}$ using Eq. (3.41), and its distribution to the affected LBM nodes,

- updating the fluid velocity after taking into account the feedback effect of the particles by solving the complete LBM equations for the fluid phase.

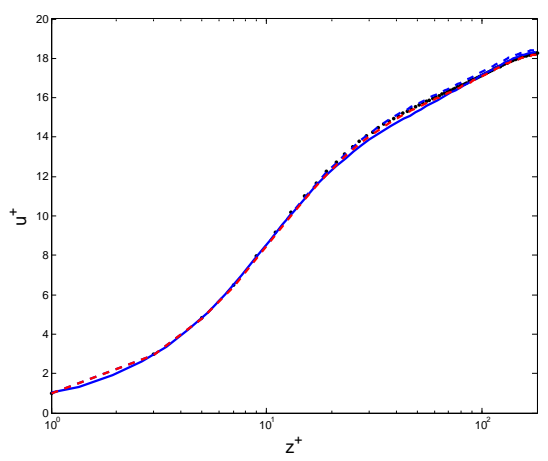

Figure 3.5: Validation of turbulent channel flow simulations. Mean streamswise velocity: KMM (solid-blue line), case A (dotted-black line), case B (dashed-blue line), case $\mathrm{C}$ (dashed-red line). 


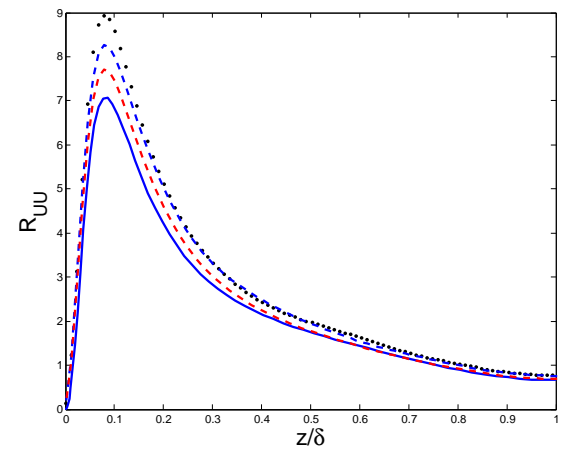

Figure 3.6: Validation of turbulent channel flow simulations. Reynolds shear stress $-\overline{u^{\prime} u^{\prime}}$, normalized by the wall shear velocity: KMM (solid-blue line), case A (dotted-black line), case B (dashed-blue line), case C (dashed-red line).

\subsection{Results}

\subsubsection{Validation of turbulent channel flow simulations}

Before modeling particle-laden turbulent channel flows, we first assess the accuracy and convergence of LBM results for a single fluid phase by solving the case, already parameterized above, corresponding to the reference DNS study of Kim et al. [19] (KMM). Because they used a variable grid in their finite volume model, KMM solved the flow in a larger channel, $8 \delta \times 4 \delta \times 2 \delta$, while in our case, when solving for particle motions, the memory limitation of using a single GPGPU yielded a maximum size $2.9 \delta \times 2.9 \delta \times 2 \delta(260 \times 260 \times 180$ lattice nodes; referred to as case A). Larger sizes/grids, however can be solved in the absence of particles.

Thus, to investigate the convergence of flow fields towards KMM's results, we ran three DNSs for a single fluid, using varying grid sizes, while maintaining the distance between walls to $2 \delta$ as in KMM. Besides case A, we modeled a larger channel in case $\mathrm{B}$, with dimensions of $3.9 \delta \times 3.4 \delta \times 2 \delta(350 \times 300 \times 180$ lattice nodes; 18.9 million), which is about the largest size achievable on one GPGPU 

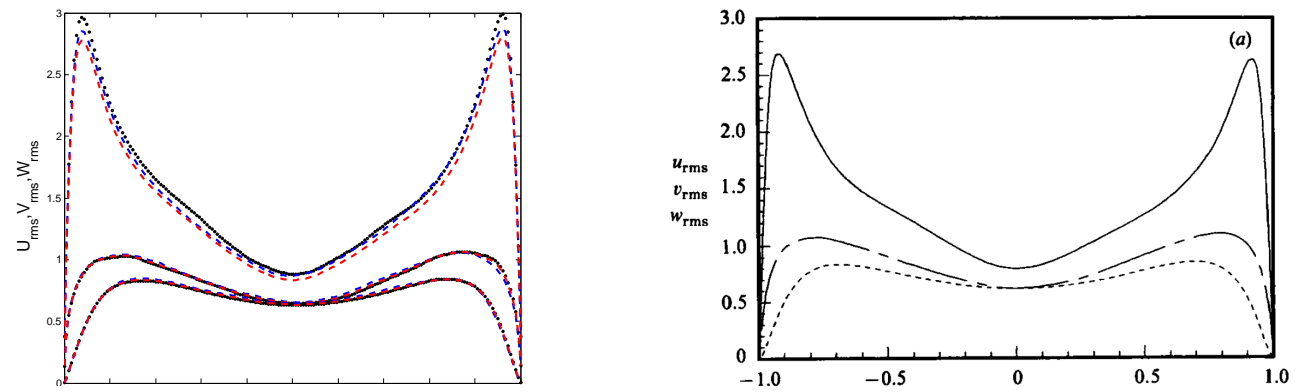

Figure 3.7: Validation of turbulent channel flow simulations. Root-mean-square velocity fluctuations normalized by the wall shear stress, : KMM (solid-blue line), case A (dotted-black line), case B (dashed-blue line), case C (dashed-red line).

without particles, and finally the largest grid in case $\mathrm{C}$, with $6.7 \delta \times 3.4 \delta \times 2 \delta$ $(600 \times 300 \times 180$ lattice nodes; 32.4 million), for which we used two GPGPUs. Note, this single attempt at using 2 GPGPUS is only applicable to single fluid flows, and this model has not yet been extended to particle-laden flows, for which the management of particles moving between GPGPU regions and flow interpolation is much more complicated.

Figures 3.5, 3.6, and 3.7 show the mean streamwise velocity, normalized Reynolds stresses, and root-mean-square velocities computed for cases A, B, C, respectively in comparison to KMM's results. While differences for the mean streamwise velocity are quite small for all cases, for the turbulent properties, as should be expected LBM results obtained in the larger domains $\mathrm{B}$ and $\mathrm{C}$ are in better agreement with KMM's benchmark study, with case C results having nearly converged to those of KMM. Even for case A, however, results are in reasonable agreement with KMM's results, which will allow using this case for studying effects of the dispersed particle phase on turbulent flow properties, particularly since this will be based on comparing results with and without particles, for the same 
domain (case A). Clearly, however, more accurate results would be obtained for larger grids, which all require using several GPGPUs for the particle-laden flow simulations as well. This will be left for future work.

\subsubsection{Turbulent particle-laden flow simulations}

As explained above, these simulations are performed in the grid case A. Four non-dimensional parameters have been previously identified, that govern the dynamics of turbulent particle-laden flows. In the present simulations, we kept the flow Reynolds number and particle density ratio constant, while varying the concentration (mass fraction) and size (Stokes number) of particles. Table 3.1 summarizes the different cases that were simulated. Numerical results for each case will be presented and discussed in the following sections. Figures will be presented as a series of curves corresponding to the various cases, obtained first by varying the mass fraction (at constant Stokes number), and then by varying the Stokes number (keeping the mass fraction constant). Line codes in figure legends will be kept identical throughout and are listed in Table 3.2 .

\begin{tabular}{lccccc}
\hline Case & $N_{p}$ & $\Phi_{p m}$ & $\mathbf{S t}^{+}$ & $d_{p}(\mu \mathrm{m})$ & $d_{p}^{+}$ \\
\hline Clean channel & 0 & - & - & - & - \\
I & $6 \times 10^{5}$ & 0.25 & 10 & 26 & 0.104 \\
II & $4.6 \times 10^{4}$ & 0.10 & 30 & 45 & 0.18 \\
III & $1.2 \times 10^{5}$ & 0.25 & 30 & 45 & 0.18 \\
IV & $2.3 \times 10^{5}$ & 0.5 & 30 & 45 & 0.18 \\
V & $2.3 \times 10^{4}$ & 0.25 & 90 & 78 & 0.312 \\
VI & 985 & 0.25 & 720 & 220 & 0.88 \\
VII & 1,980 & 0.5 & 720 & 220 & 0.88 \\
\hline
\end{tabular}

Table 3.1: Turbulent particle-laden flow simulations. Particle properties for seven different DNSs, using $\mathbf{R e}=180$ and $D_{\rho}=1000$. The particle diameter $d_{p}^{+}$has been normalized using the viscous length scale. 


\subsubsection{Mean and r.m.s. flow velocity}

The effects of particles on the turbulence can be observed on the mean streamwise velocity of the carrier fluid across the channel width, shown in Fig. 3.8 in semi-log scale and in Fig. 3.9 in linear scale. In the presence of particles, the mean flow velocity decreases in the logarithmic region and near the center of the channel relatively to the clean channel case, while it is nearly unchanged near the walls.
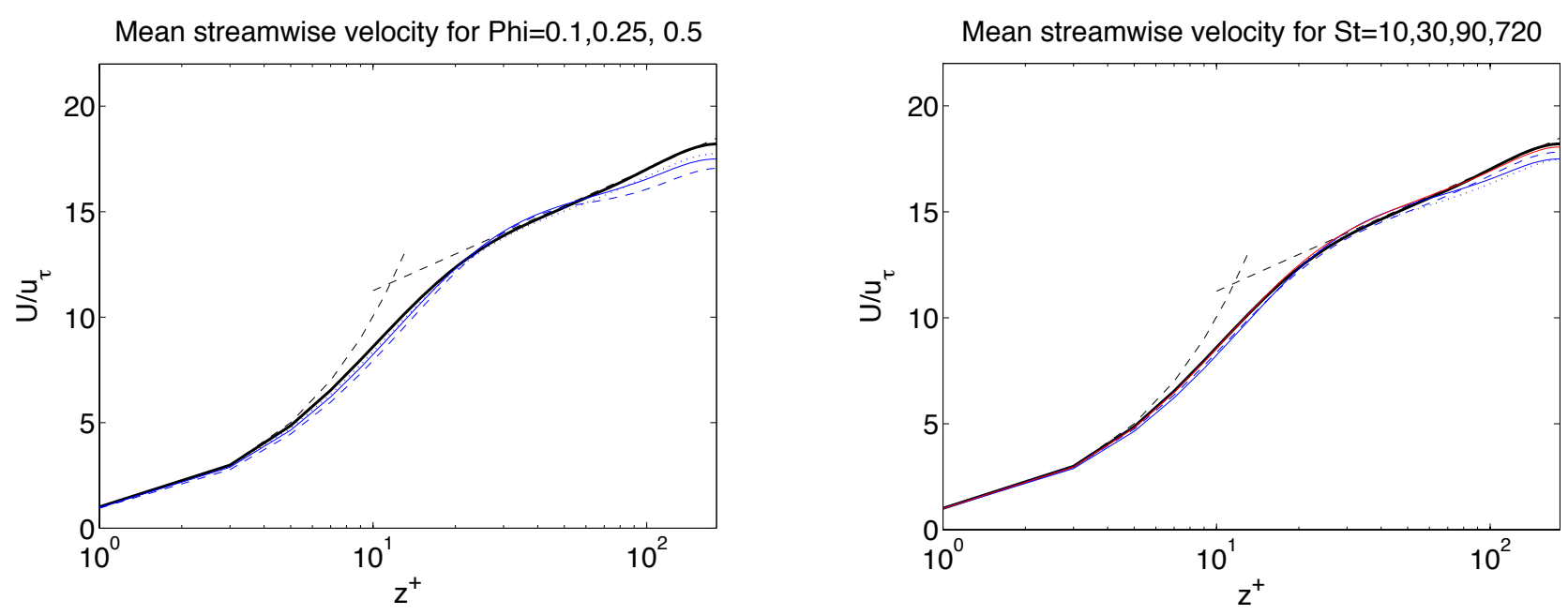

Figure 3.8: Turbulent particle-laden flow simulations. Mean streamwise velocity (log scale), normalized by the friction velocity. Left: as a function of increasing mass fraction: cases II, III, IV $\left(\mathbf{S t}^{+}=30\right)$; Right: as a function of increasing Stokes number: cases I, III, IV, VI $\left(\Phi_{m}=0.25\right)$.

\begin{tabular}{lcr}
\hline DNS runs & Case & Color, symbol \\
\hline \hline Baseline & Clean channel & black solid line \\
\hline \multirow{3}{*}{ Effects of concentration } & II & blue dot line \\
& III & blue solid line \\
& IV & blue dash line \\
\hline \multirow{2}{*}{ Effects of particle size } & I & blue dot line \\
& III & blue solid line \\
\multirow{2}{*}{ Effects of large particles } & V & blue dash line \\
& VII & red solid line \\
& red dash line \\
\hline
\end{tabular}

Table 3.2: Turbulent particle-laden flow simulations. Line codes in figure legends for simulation cases listed in Table 3.1 . 

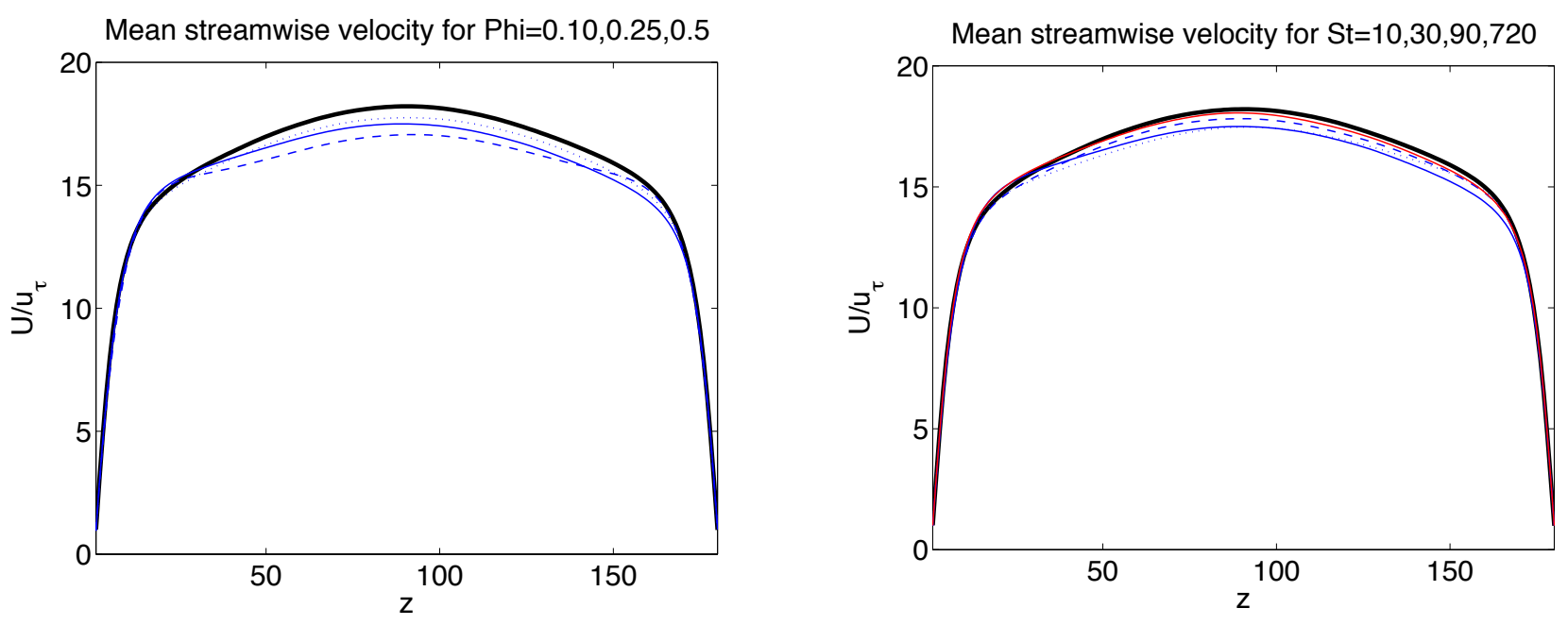

Figure 3.9: Turbulent particle-laden flow simulations. Same results as in Fig. 3.8 in linear scale.

More specifically, for particle-laden flows, the mean velocity profiles are all found to be below the baseline profile from $z^{+} \approx 20$ (i.e., beyond the viscous wall region) to the center of the channel, while they remain very close to the clean channel profile near the wall. In the case where the mass fraction increases from 0.1 to 0.25 and then to 0.5 (left panels), a consistent decrease of the velocity can be noted, with the largest difference occurring for case IV. The right panels indicate that the discrepancy with the baseline (clean channel) is largest for case I (i.e., the smallest particles) and is reduced for the larger particles. The impact nearly disappears for case VI $\left(\mathbf{S t}^{+}=720, \Phi_{p m}=0.25\right)$. This suggests that the smaller particles (for the particle size range investigated) are more effective in modifying the mean profile, given a constant mass fraction. Among all the cases, the case IV $\left(\mathbf{S t}^{+}=30, \Phi_{m}=0.5\right)$ shows the most impact on $\langle u\rangle$.

Next, Fig. 3.10 shows the root-mean-square of turbulent velocity fluctuation components computed across the channel. This is the primary statistics of turbulence, where effects of particles can be easily observed. In general, the presence of particles increases the streamwise turbulence intensities, while reducing 
the spanwise and the wall-normal components. For increasing particle concentration, the streamwise fluctuations are larger relative to the baseline of the clean channel. The increase is non-monotonic, however, and happens mainly outside the viscous sublayer, after the maximum of the fluctuations has been reached (around $z / \delta \approx-0.9)$. The spanwise and wall-normal fluctuations noticeably drop across the channel width, as compared to the baseline, and the maximum of each curve is displaced further away from the wall. The disparity with the clean channel becomes more pronounced when a larger number of particles is dispersed in the flow. For increasing Stokes number, the streamwise velocity fluctuations are higher than for the clean channel, though the difference is negligible for the case VI. The enhancement of the streamwise fluctuations is particularly evident for cases III and V. Dampening of the $y$ - and $z$-fluctuation components can be seen in all cases. Moreover, case I with the smallest particles displays an interesting behavior: the curves for $v_{r m s}$ and $w_{r m s}$ start at the wall between cases III and V, but end up crossing the curves beyond the viscous wall region.

Overall, we may conclude that there is a global effect of $\Phi_{p m}$ and of $\mathbf{S t}^{+}$ on the lower-order statistics of turbulence, though the underlying mechanisms of turbulence modulation may be different whether $\mathbf{S t}^{+}$or $\Phi_{p m}$ are changed in the DNS runs. According to Zhao et al. [9], it is thought that the inertia of particles may play an important role in modifying the low-order moments of turbulence, at any concentration. The attenuation of the wall-normal fluctuations, combined with the enhancement of the streamwise turbulence intensities, has a profound impact on many aspects of turbulent channel flows.

\subsubsection{Streamwise force balance}

One way to verify that the two-way coupling approach is correctly implemented in the LBM model is to compute a stream-wise force balance between 

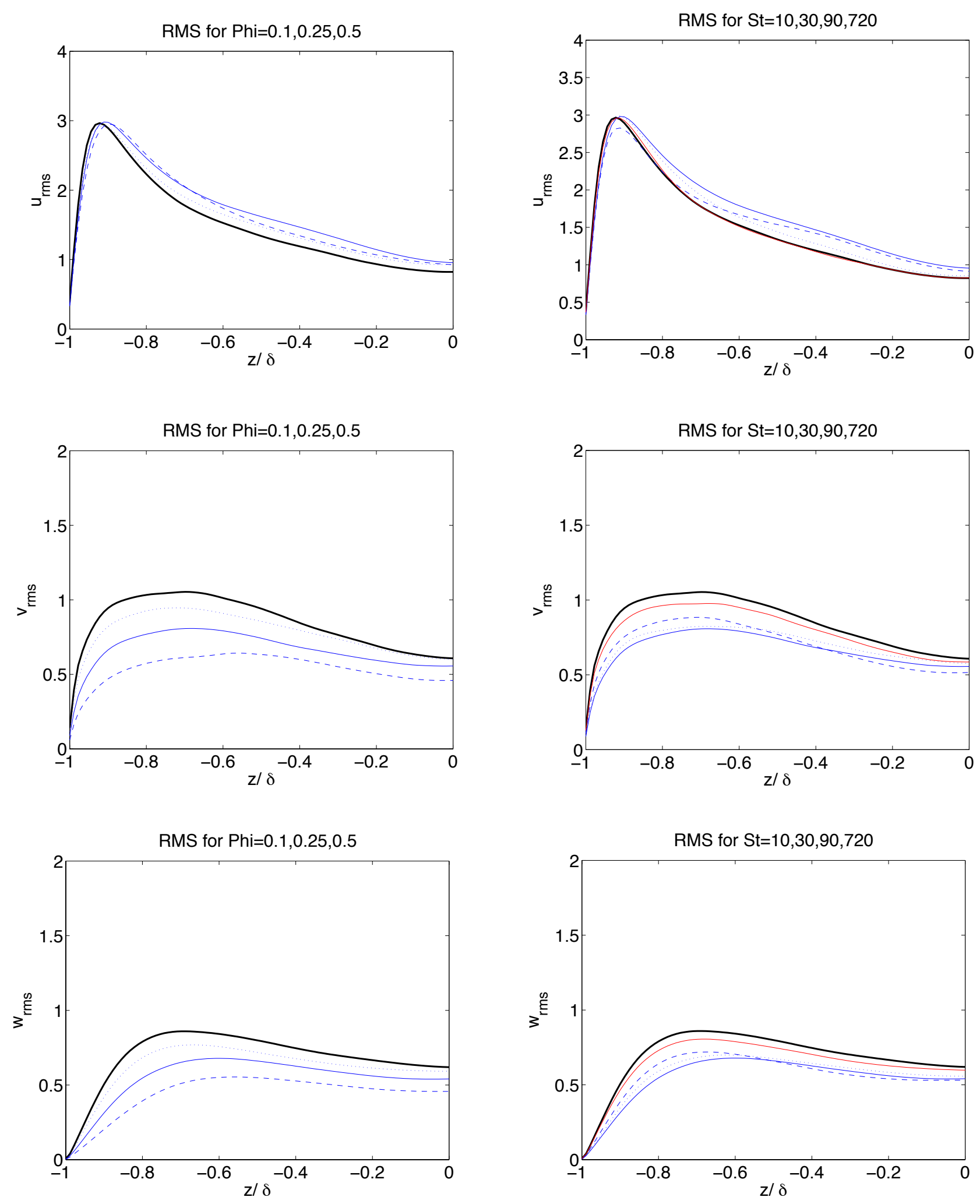

Figure 3.10: Turbulent particle-laden flow simulations. Root-mean-square velocity fluctuations. Top: streamwise, middle: spanwise, bottom: wall-normal. Left: increasing mass fraction, right: increasing Stokes number. See Fig. 3.8 for details of cases. 
the feedback volume force, which is included in the LBM equation, as a result of momentum exchanged between the fluid and the dispersed phase and other components of the momentum balance, in the stream wise $x$ direction. Once we verify that the conservation of momentum is satisfied, we can focus on understanding the particles' role in the global force balance. The "mean" operator (denoted by the angle brackets) here refers to results that are both spatially averaged in the streamwise lattice direction and time averaged over the simulation.

In the case of the clean channel, the force balance consists of the mean viscous force and the Reynolds force, which should be exactly compensating the mean pressure gradient,

$$
F_{V}+F_{R}=\frac{\partial\langle p\rangle}{\partial x}, \text { with } F_{V}=\frac{\partial}{\partial z}\left(\mu \frac{\partial\langle u\rangle}{\partial z}\right) \text { and } F_{R}=-\frac{\partial}{\partial z}\left(\rho_{f}\left\langle u^{\prime} w^{\prime}\right\rangle\right)
$$

Note that while many studies examine the stress balance and compute the viscous and Reynolds stresses in turbulent Poiseuille DNS, examining the force balance is more relevant in the case where particles are dispersed in the computational domain. We emphasize that both formulations are equivalent, and we can relate the forces described in Eq. (3.42) to the viscous and Reynolds stresses as,

$$
\tau_{V}=\mu \frac{\partial\langle u\rangle}{\partial z} \text { and } \tau_{R}=-\rho_{f}\left\langle u^{\prime} w^{\prime}\right\rangle
$$

In the case of particle-laden flows, the force balance becomes:

$$
F_{V}+F_{R}+F_{p}=\frac{\partial\langle p\rangle}{\partial x}, \text { with } F_{p}=\rho_{f}\left\langle B_{1}\right\rangle
$$

where $B_{1}$ denotes the $x$-component of the particle feedback volume force computed with Eq. (3.41).

Figure 3.11 shows the repartition of the various forces for channel flow simulations, as a function of the normalized distance from the wall. [Note that the legend in this figure differs from that of the previous plots.] The first plot corresponds 
to the clean channel case, where we see that the sum of the viscous and Reynolds forces nearly exactly compensate the mean pressure gradient applied to the flow (within a small numerical error). This shows that there are no sink or source of momentum in the channel flow DNS, confirming the accuracy of the LBM solution. The second plot illustrates the repartition of the forces among viscous, Reynolds, and particle feedback forces, for cases II, III and IV (for increasing particle concentration), while the third plot shows a similar family of curves, for cases I, III, and V (for increasing Stokes number). The last plot, on the lower right, shows the force components, for the case with the largest concentration of particles (case IV).

As expected, for the clean channel case, the absolute value of the viscous force is maximal in the viscous layer [18], and reaches zero beyond the buffer layer $\left(z^{+} \approx 30\right)$. The Reynolds force has an opposite sign to the viscous force, and balances it by reaching the value of the mean pressure gradient at the point where viscous effects vanish away from the wall. The effect of an increasing particle mass fraction on the force balance can be seen in the second plot, in the upper right corner: inside the viscous sublayer the magnitude of both the viscous force and the Reynolds force progressively decreases, while the particle force $\left\langle B_{1}\right\rangle$ increases at the same time, and gradually more the larger $\Phi_{p m}$. A small discrepancy between the sum of the forces and the mean pressure gradient is observed near the wall, which is likely due to the treatment of the no-slip boundary condition in the LBM (bounce-back scheme). Similar observations can be made in the third plot (lowerleft) for the forces and force balance for the case of an increasing Stokes number. Again, the magnitude of the forces are smaller than for the clean channel case. However, the evolution of the forces is non-monotonic: the curves for case I are found to be in between cases III and V (Reynolds force) and case V (viscous 
force). The lesser impact of particles is seen for case VI (not plotted here), which is expected since it corresponds to the smallest amount of particles. In both cases (second and third plot), besides the numerical artifact near the wall, it is clear that the force balance is well satisfied across the channel width in the presence of dispersed particles, thus confirming the relevance and accuracy of the proposed LBM-DNS approach for two-way coupled particle-fluid problems.

Additional observations are that the Reynolds force decreases faster than the viscous force near the wall for increasing mass fraction or Stokes number. On the other hand, for cases II, III and IV, the locations of the particle force maxima are closer to the wall than the maxima of both $F_{V}$ and $F_{R}$, which indicates that the particles impact the turbulence mainly close to the wall, rather than in a homogeneous fashion over the whole domain. While the particle force magnitude can reach up to five times that of the mean pressure gradient, it remains very localized in the domain. Outside the viscous sublayer the particle force is small but remains finite, and reduces the Reynolds force accordingly. Lastly, the fourth plot in Fig. 3.11 on the lower right shows the mean particle force component variation across the channel for Case IV. As expected, $\left\langle B_{1}\right\rangle$ in the streamwise direction provides the maximum contribution while $\left\langle B_{3}\right\rangle$ is zero everywhere. It is interesting to note that $\left\langle B_{2}\right\rangle$ is not zero near the wall, but its magnitude is much smaller than that of $\left\langle B_{1}\right\rangle$.

From Fig. 3.11, we may conclude that regardless of their size or concentration, small inertial particles allowed to interact directly with the carrier fluid impact the distribution of the forces by dampening the Reynolds force (across the channel) and the viscous force (within the viscous wall region). 

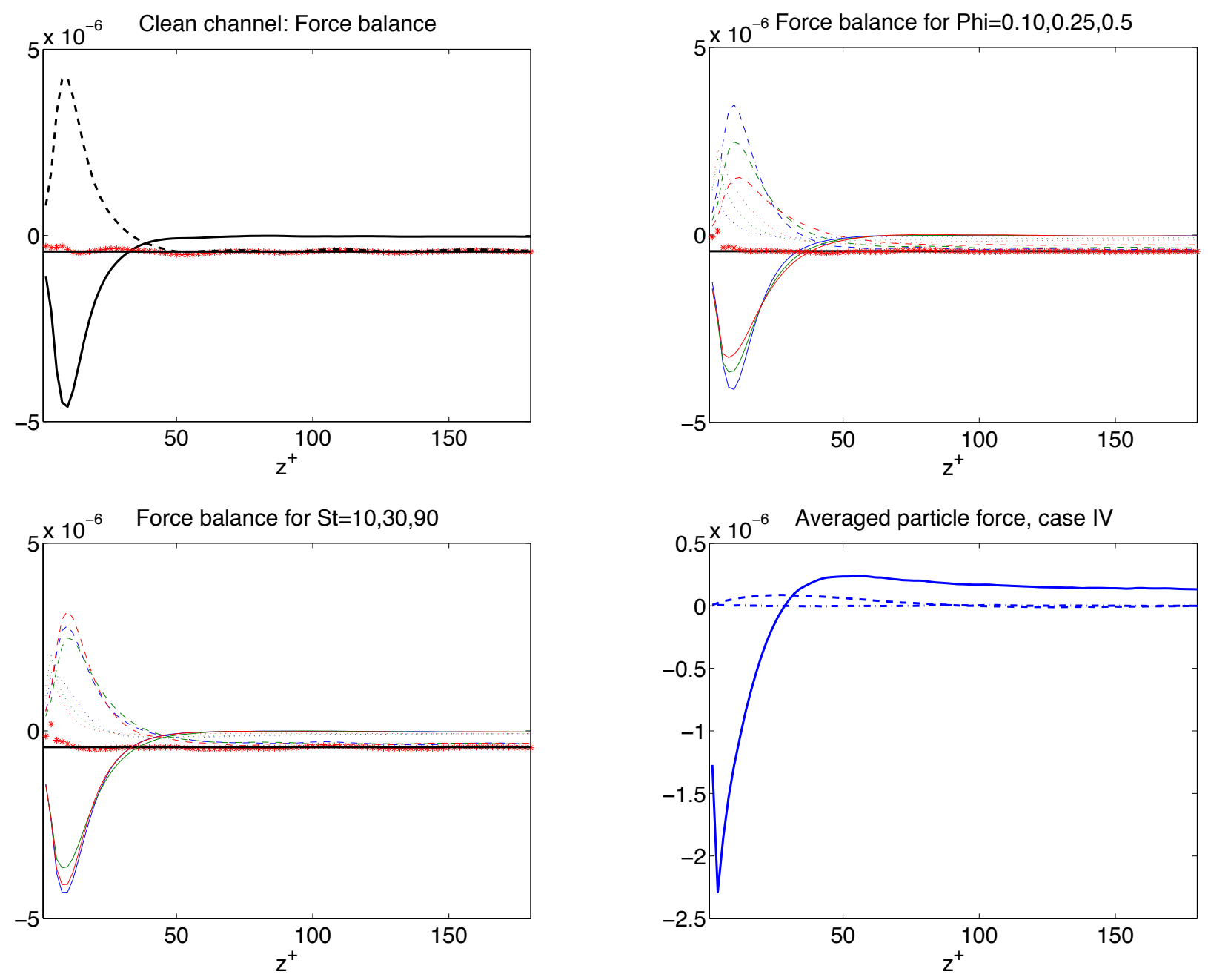

Figure 3.11: Turbulent particle-laden flow simulations. Average force balance in streamwise direction: clean channel (upper left); varying mass fraction (upper right: case II-blue, III-green, IV-red); varying Stokes number (lower left: case Iblue, III-green, V-red); and particle force components for case IV (lower right). Legend for first 3 plots: viscous force $F_{V}$ (solid line), Reynolds force $F_{R}$ (dash line), particle feedback force $F_{p}$ (dotted line), pressure gradient $\partial\langle p\rangle / \partial x$ (black thin horizontal line marked at at $-4.34 \times 10^{-7} M L^{-2} T^{-2}$ ), and sum of all forces (red starred line). Legend for last plot: $\left\langle B_{1}\right\rangle$ (solid line), $\left\langle B_{2}\right\rangle$ (dash line), $\left\langle B_{3}\right\rangle$ (dashdotted line).

\subsubsection{Production and dissipation of mean TKE}

Zhao et al. [9] investigated particle effects on the mean turbulent kinetic energy (TKE) budget of particle-laden fluid flows, focusing on the energy transfer between the fluid and the particle phases and on the dissipation rate in the wall 
turbulence. This budget is based on a conservation equation that can be derived from the NS Eqs. (3.21) and 3.22 as,

$$
\begin{aligned}
\frac{D k}{D t}= & -\frac{1}{\rho_{f}} \frac{\partial\left\langle u_{i}^{\prime} p\right\rangle}{\partial x_{i}}-\frac{\partial\left\langle k u_{i}^{\prime}\right\rangle}{\partial x_{i}}+\nu \frac{\partial^{2} k}{\partial x_{j} \partial x_{j}} \\
& -\left\langle u_{i}^{\prime} u_{j}^{\prime}\right\rangle \frac{\partial\left\langle u_{i}\right\rangle}{\partial x_{j}}-\nu \frac{\partial\left\langle u_{i}^{\prime} u_{j}^{\prime}\right\rangle}{\partial x_{j} \partial x_{j}}+\left\langle u_{i}^{\prime} B_{i}^{\prime}\right\rangle .
\end{aligned}
$$

with $k=(1 / 2)\left\langle u_{i}^{\prime} u_{i}^{\prime}\right\rangle$ the mean TKE, in which there are 7 terms, from left to right, the mean TKE: (1) time rate of change, (2) pressure transport, (3) turbulent transport, (4) viscous diffusion, (5) production, (6) dissipation rate, and (7) particle production.

For a statistically stationary turbulent channel flow, the mean TKE is conserved and its time rate of change, which is expressed in the left-hand-side of Eq. (3.44) by a material derivative, corresponding to the sum of a local rate of change and the advection of $k$ by the mean flow, must be equal to zero. Since gravity has been neglected in this work, buoyancy does not intervene in the mean TKE balance. Here, we are mainly interested in the repartition of mean TKE among production terms and the dissipation rate. We will denote the mean TKE production by $P$, particle production by $P_{p}$ and dissipation rate by $\varepsilon$. In wall-bounded turbulent flows, the production term $P$ can be simplified to,

$$
P=\left\langle u^{\prime} w^{\prime}\right\rangle \frac{\partial\langle u\rangle}{\partial z}
$$

Figure 3.12 plots $P, P_{p}$ and $\varepsilon$ across the channel width, for cases I to VI, with the clean channel results being shown as a baseline. The left-side figures represent cases II, III and IV with increasing mass fraction; due to particle feedback effects particles, we see a reduction of the TKE mean production and the presence of a non-zero production term $P_{p}$. The magnitude of the dissipation rate also decreases with increasing mass fraction. The top and bottom panels show that the particle production is small compared to the TKE production, and negative across the 
channel width for the cases III and IV. The fact that $P_{p}$ becomes negligible for case II is due to the very low concentration of particles dispersed in the flow. The right-side figures represent cases with increasing Stokes number; we see that particles of various diameters impact in a different and complex manner the TKE production and dissipation terms, although both the production and dissipation terms are reduced in the presence of particles. The impact is much smaller however for case VI $\left(\mathbf{S t}^{+}=720, \Phi_{p m}=0.25\right)$ which has a very small number of number of particles. We also see that the particle production term changes sign for some cases, being mostly negative for cases I and III, then positive across the channel width for case V, and finally nearly zero for case VI, where as indicated, very few particles were dispersed in the flow (like in case II).

To summarize, the introduction of many small particles in a turbulent channel flow modifies the TKE budget across the channel width, in the wall-normal direction, although change is not monotonic and varies depending on values of the mass fraction and Stokes parameters. The fine turbulent flow structures that influence the TKE production and dissipation are further discussed in the next section.

\subsubsection{Turbulent coherent structures}

It is commonly accepted in the wall turbulence research community that turbulence appears in the flow as organized structures, spanning several length and time scales [25]. According to Adrian [26], wall turbulence is characterized by the presence of packets of hairpin vortices and associated quasi-streamwise vortices (QSV) near the walls. Such turbulent structures, which are referred to as coherent because they persist for a long time in the flow, have been extensively studied both experimentally and numerically. In particular, a mechanism for generating QSVs in a turbulent channel flows was suggested by Zhou et al. [27. They argued that the turbulent boundary layer contains a large number of hairpin 

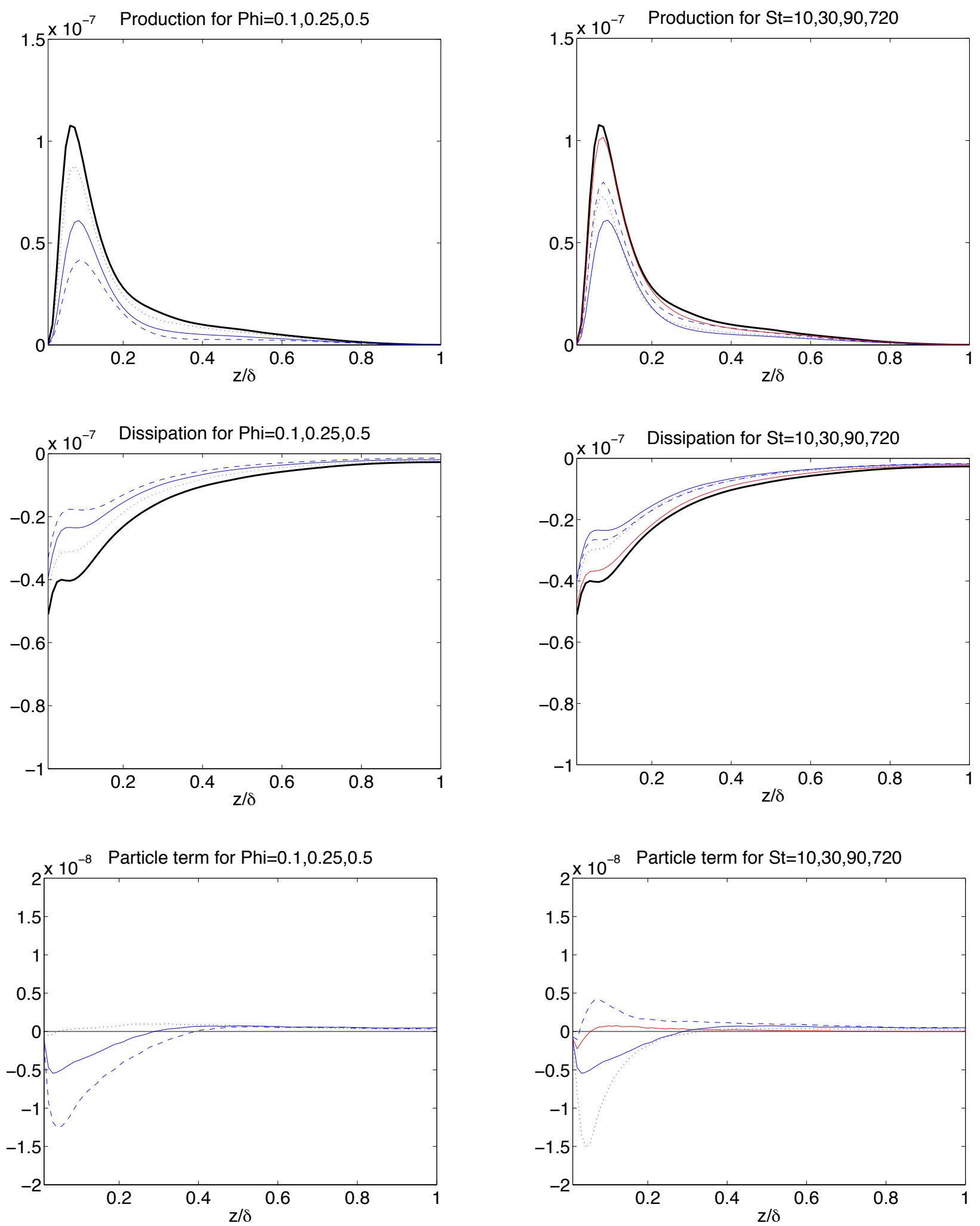

Figure 3.12: Turbulent particle-laden flow simulations. TKE production and dissipation terms, across the channel width. Top: $P$, middle: $\varepsilon$, bottom: $P_{p}$. Left: cases with increasing mass fraction, right: cases increasing Stokes number. See definitions in Table 3.2 . 
vortices, aligned in the streamwise direction as coherent packets. Although they first studied the evolution of a single idealized symmetric hairpin vortex, they also investigated asymmetric hairpin vortices and concluded that QSVs generated from asymmetric structures occurred more often singly and rarely as counter-rotating pairs of equal strength. Zhou et al. added that asymmetric vortex generation was more frequently observed experimentally in turbulent boundary layers. Jeong et al. 28] investigated the role of near wall coherent structures in turbulent channel flows and proposed a model with overlapping and alternating-sign QSVs, as the dominant near-wall coherent structures (Figure 3.13). Note, similar to KMM's study, the convention used in this figure for the coordinates is that the $y$-direction corresponds to the wall-normal direction.

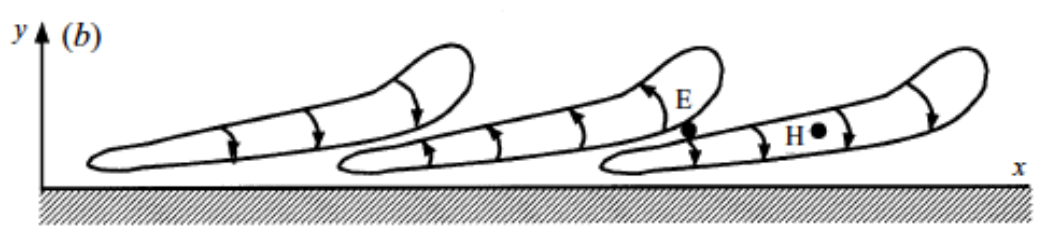

Figure 3.13: Schematics of an array of quasi-streamwise vortices (QSV) (from Jeong et al. [28]).

Figure 3.14 shows instantaneous snapshots of streamwise velocity $u$ from a side view $(x-z)$, as well as the second component of vorticity $\omega_{y}$ (rotation around our $y$ direction). Vorticity is computed by a finite difference scheme, based on its 

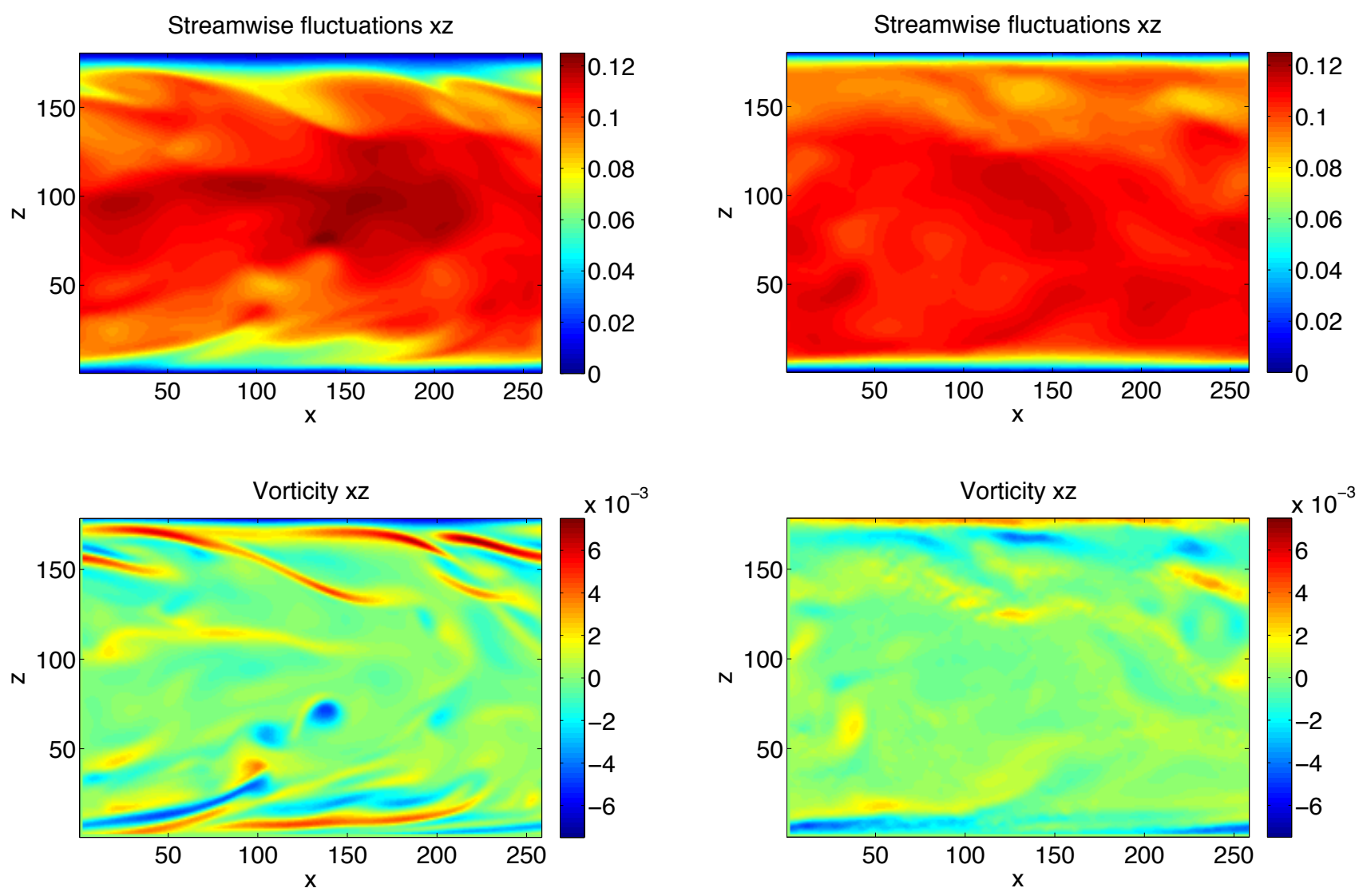

Figure 3.14: Turbulent particle-laden flow simulations. Instantaneous snapshots of: (up-left) streamwise velocity $u$ for the clean channel, (up-right) streamwise velocity for case IV, (bottom-left): vorticity $\omega_{y}$ (rotation around $y$ direction) for the clean channel, and (bottom-right) vorticity for case IV.

general definition,

$$
\omega_{i}=\epsilon_{i j k} \frac{\partial u_{k}}{\partial x_{j}},
$$

with $\epsilon_{i j k}$ being the 3rd-order antisymmetric unit tensor. For channel flow turbulence, where the flow is strongly anisotropic, the vorticity component of interest is,

$$
\omega_{y}=\frac{\partial u}{\partial z}-\frac{\partial w}{\partial x}
$$

which can be computed based on LBM results at various lattice nodes at a given 
time as,

$$
\begin{aligned}
\omega_{y}= & \frac{1}{10 \Delta x}\left(2 w_{i+2, j, k}+w_{i+1, j, k}-w_{i-1, j, k}-2 w_{i-2, j, k}\right) \\
& -\frac{1}{10 \Delta z}\left(2 u_{i, j+2, k}+u_{i, j+1, k}-u_{i, j-1, k}-2 u_{i, j-2, k}\right),
\end{aligned}
$$

Snapshots in Fig. 3.14 clearly capture the dominant turbulent structures in the computational domain. In particular, for the clean channel flow, the pattern of vorticity seen in the lower left corner figure matches the locations of slower streamwise velocity in the upper left corner plot. Comparing these simulations to the schematics of Jeong et al. 28] in Fig. 3.13, suggests that the structures of positive and negative vorticity $\omega_{y}$ indeed correspond to QSVs, or more generally to turbulent coherent structures. In the upper left corner plot, the enforced noslip boundary condition results in a thin blue layer near each wall, where the streamwise velocity $u \approx 0$. Conversely, the velocity is maximum near the center of the channel. The upper right plot shows, for particle laden flows, the striking absence of the coherent structures near the walls, as well as a reduction of the velocity at the centerline of the channel. Vorticity in the lower right plot is also significantly reduced, except for a few traces of negative vorticity near the walls. It is evident that the major effects of small inertial particles in the flow is to dampen the turbulent coherent structures near the boundaries of the channel.

Patterns observed for the streamwise velocity and QSVs are further investigated in Fig. 3.15 where left-side plots are for the clean channel and right-side plots are case IV particle-laden flow. The upper plots show the streamwise velocity $u$ observed from a top view $(x-y$ plane $)$ at a small distance from the channel bottom wall, while the lower plots show $u$ at the mid-channel width. The scale of velocity at the channel center (from 0.1 to 0.12 ) takes into account the fact that the velocity at the center of the channel has a large mean value and smaller fluctuations. Consistent with Fig. 3.14, in the upper plots, we see that turbulent 

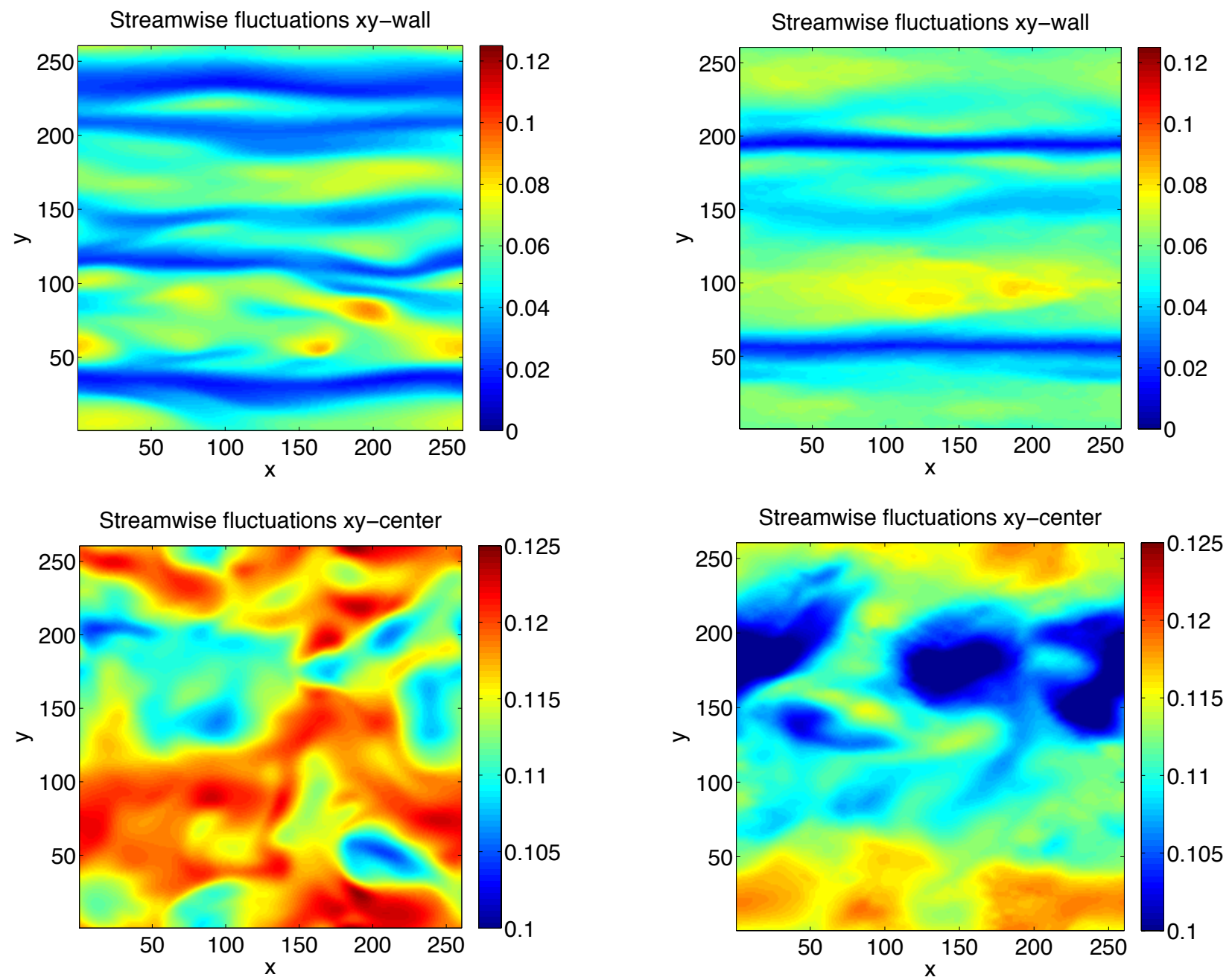

Figure 3.15: Turbulent particle-laden flow simulations. Snapshots of velocity fluctuations in horizontal $(x-y)$ planes. Clean channel flow (left plots), case IV (right plots)

structures near the wall are drastically modified by the particles. The vortices present in the clean channel flow are weakened under the action of particles, leading to less low-speed regions near the wall. In the lower plots the effects of particles on turbulent structures are less obvious, other than the fact that the mean velocity is slightly reduced. 


\subsection{Summary and conlusions}

In this work, we efficiently implemented, on a heavily parallel GPGPU board, a 3D-LBM model for DNS of turbulent particle-laden flows and used it to simulate and study a variety of channel flow turbulent properties, as a function of various mass fractions, numbers, and sizes of particles.

We first validated the fluid-only model by simulating turbulent boundary layer flows, in a periodic channel with two solid walls without particles, and comparing results to those of the reference paper by Kim et al. [19]. We concluded that, despite using a smaller computational domain in the wall-parallel directions than normally necessary to resolve all the turbulent eddies, our results were in good agreement with KMM's results, in particular for statistics of turbulent fields (e.g., mean velocity, normalized Reynolds stresses). In the absence of particles, larger computational domains were used on a single GPGPU and even larger using two GPGPUs, that showed a clear convergence of LBM towards KMM's results. In the presence of particles, the LBM grid has to be smaller on the single GPGPU and clearly this is a limitation of present simulations. Future work could extend the simulation of particle-laden flows to multiple GPGPUs. Nevertheless, this validation study confirmed that turbulent flow properties were adequately simulated in the selected model grid, particularly in the across wall boundary layer direction, to allow us accurately study particle effects on turbulent properties and structures.

Hence, next, we explored the effects of having a dispersed phase made of many small inertial particles in turbulent channel flows. Once particles were properly dispersed in the flow, we performed several DNS runs by varying the particle concentration and size (i.e., the mass fraction: $\Phi_{m}$, and the viscous Stokes number $\mathbf{S t}^{+}$). Overall, compared to the clean channel, these DNSs showed that effects of particles are to reduce the mean streamwise velocity, turbulent structures and 
intensity near the walls, and spanwise and wall-normal velocity fluctuations, while accentuating streamwise turbulent intensities and velocity fluctuations.

The momentum conservation and force balance were computed as well. In the clean channel case, the Reynolds and viscous forces compensate each other, so that their sum equals the mean pressure gradient applied to sustain the mean flow. With particles (cases I-VI), feedback force appears in the force balance, and a new force distribution takes place: both the Reynolds and viscous forces are much lower than in the clean channel case, while the particle force progressively increases for larger mass fractions and decreases for larger Stokes number (since a lower number of particles is required to reach the given mass fraction of 0.25 for cases V and VI) . Additionally, the turbulent kinetic energy (TKE) budget, as well as the mean-shear production, dissipation rate and particle production were evaluated. The particle production is usually small relative to the flow production term, and can change sign depending on the particle size (cases I and III: $P_{p}<0$, case V: $P_{p}>0$, case VI: $\left.P_{p} \approx 0\right)$. Both $P$ and $\varepsilon$ are found smaller than for the clean channel case, in all six cases studied with particles, implying that the general effect of particles on turbulence is to reduce its production and attenuate the dissipation. Finally, we investigated the interactions between the turbulent coherent structures and the dispersed phase. From the various snapshots of instantaneous velocity and vorticity, we may argue that the coherent structures (quasi-streamwise vortices and sweeps) are weakened near the channel walls in presence of particles.

\section{List of References}

[1] S. Elghobashi, On predicting particle-laden turbulent flows. Applied Scientific Research (1994) 52, 309-329

[2] S. Balachandar, J.K. Eaton, Turbulent dispersed multiphase flow. Annual Review of Fluid Mechanics (2010), 42, 111-133 
[3] M. Rashidi,G. Hetsroni, S. Banerjee, Particle turbulence interaction in a boundary layer. Intl J. Multiphase Flow (1990) 16, 935-949

[4] K.D. Squires, J.K. Eaton, Particle response and turbulence modification in isotropic turbulence. Phys. Fluids (1990) A2, 1191-1203

[5] Y. Pan, S. Banerjee, Numerical simulation of particle interactions with wall turbulence. Phys. Fluids (1996) 8, 2733-2755.

[6] Y. Li, K. Kontomaris, J. B. McLaughlin. DNS of Particle-Laden Channel Flows with Feedback, American Institute of Chemical Engineers (1999).

[7] D.H. Richter, P.P. Sullivan, Momentum transfer in a turbulent, particle-laden Couette flow. Physics of Fluids, (2013), 25

[8] D.H. Richter, P.P. Sullivan, Sea surface drag and the role of sea spray. Geophysical Research Letters, (2013) 40, 656-660

[9] L. Zhao, H.I. Andersson, J.J. Gillissen, Interphasial energy transfer and particle dissipation in particle-laden wall turbulence. J Fluid Mech (2013) 715, $32-59$

[10] J. Tölke, M. Krafczyk, Implementation of a lattice Boltzmann kernel using the compute unified device architecture developed by nVIDIA. Computing and Visualization in Science (2008) 1, 29-39.

[11] Y. Qian, d'Humieres, Lallemand, Lattice BGK models for Navier Stokes equation. Europhysical Letters (1992) 17, 479-84.

[12] D. Yu, R. Mei, L. Luo, W. Shyy, Viscous flow computations with the method of lattice Boltzmann equation. Progress in Aerospace Sciences (2003) 39, 329 367

[13] C. Janßen, M. Krafczyk, Free surface flow simulations on GPGPUs using LBM. Computers and Mathematics with Applications (2011) 61 (12), 35493563.

[14] J. Buick, C. Greated, Gravity in lattice Boltzmann models. Physical Review (2000) E61, 5307-5320.

[15] M. Krafczyk, J. Tölke, L.S. Luo, Large-eddy simulations with a multiplerelaxation-time LBE model. Intl. J. Modern Phys. (2003) B17(01-02) , 33-39

[16] D. DHumieres, I. Ginzburg, L. Luo, Multiple relaxation time lattice Boltzmann models in three dimensions. Philosophical Transactions Royal Society of London (2002) A, 360-437 
[17] A.J.C Ladd . Numerical simulation of particular suspensions via a discretized Boltzmann equation. Part 1. Theoreticalfoundation. J Fluid Mech (1994) 271, 285-309

[18] S.B. Pope, Turbulent Flows: Cambridge University Press (2000)

[19] J. Kim,P. Moin,R. Moser, Turbulence statistics in fully developed channel flow at low Reynolds number. J. Fluid Mech. (1987) 177, 133-166

[20] D.J. Bespalko, Validation of the Lattice Boltzmann Method for direct numerical simulations of wall-bounded turbulence, $\mathrm{PhD}$ thesis (Queen's University) (2011)

[21] C.F. Janßen, Kinetic approaches for the simulation of non-linear free surface flow problems in civil and environmental engineering, $\mathrm{PhD}$ thesis (Technische Universitaet Braunschweig) (2010)

[22] R. Moser,J. Kim,N.N. Mansour, Direct numerical simulation of turbulent channel flow up to $R e=590$. Physics of Fluids (1999) 11, 943-945

[23] M.R. Maxey, J.J. Riley, Equation of motion for a small rigid sphere in a nonuniform flow. Physics of Fluids,(1982) 26, 883-889

[24] F. Lekien, J. Marsden, Tricubic interpolation in three dimensions. Int. J. Numerical Methods in Engineering, (2005) 63, 455-471

[25] J.R. Fessler, J.D Kulick, J.K Eaton,Preferential concentration of heavy particles in a turbulent channel flow. Physics of Fluids (1994) 6, 3742-3749

[26] R.J. Adrian, Hairpin vortex organization in wall turbulence. Physics of Fluids (2007) 19,

[27] J. Zhou, R.J. Adrian,S. Balachandar,T.M. Kendall, Mechanisms for generating coherent packets of hairpin vortices in channel flow. J. Fluid Mech. (1999) $387,353-396$

[28] J. Jeong,F. Hussain,W. Schoppa,J. Kim,Coherent structures near the wall in a turbulent channel flow. J. Fluid Mech. (1997) 322, 185-214 


\section{APPENDIX A}

\section{Chapman-Enskog expansion for pressure-less NS equations}

Here, we verify that the mesoscopic evolution Eq. (1.21), with the equilibrium distribution functions defined in Eqs. (1.28) to (1.31), converges to the macroscopic "pressureless" NS equations of motion (1.33), by applying the Chapman-Enskog multiple-scale expansion. The latter first performs a Taylor series expansion of the left hand side of the LB Eq. (1.21) in space and time, yielding (for $i=0, \ldots, 8)$,

$$
\begin{aligned}
g_{i}\left(\boldsymbol{x}+e_{i \alpha} \Delta t, t+\Delta t\right) & =g_{i}(\boldsymbol{x}, t)+\epsilon\left(\partial_{t}+e_{i \alpha} \partial_{\alpha}\right) g_{i}(\boldsymbol{x}, t) \\
& +\frac{1}{2} \epsilon^{2}\left(\partial_{t}+e_{i \alpha} \partial_{\alpha}\right)\left(\partial_{t}+e_{i \beta} \partial_{\beta}\right) g_{i}(\boldsymbol{x}, t)+\mathcal{O}\left(\epsilon^{3}\right),
\end{aligned}
$$

with the expansion parameter $\epsilon=\Delta t$ being small compared to the macroscopic time scales. Then, we expand the particle distribution function $g_{i}(\boldsymbol{x}, t)$ as,

$$
g_{i}=g_{i}^{(e q)}+\epsilon g_{i}^{(1)}+\epsilon^{2} g_{i}^{(2)}+\mathcal{O}\left(\epsilon^{3}\right) \text {, }
$$

with the following multiple-scale expansion for the time derivative,

$$
\partial_{t}=\partial_{t_{0}}+\epsilon \partial_{t_{1}}+\mathcal{O}\left(\epsilon^{2}\right),
$$

where $t_{0}$ denotes the "slow" (macroscopic) time and $t_{1}$ is the "fast" time.

Substituting the latter two equations into Eq (A.1) and sorting out terms in orders of $\epsilon$, we obtain,

$$
\begin{array}{r}
\epsilon\left(\partial_{t_{0}}+e_{i \alpha} \partial_{\alpha}\right) g_{i}^{(e q)}+\epsilon^{2}\left\{\partial_{t_{1}} g_{i}^{(e q)}+\left(\partial_{t_{0}}+e_{i \alpha} \partial_{\alpha}\right) g_{i}^{(1)}+\frac{1}{2}\left(\partial_{t_{0}}+e_{i \alpha} \partial_{\alpha}\right)\right. \\
\left.\left(\partial_{t_{0}}+e_{i \beta} \partial_{\beta}\right) g_{i}^{(e q)}\right\}+\mathcal{O}\left(\epsilon^{3}\right)=-\frac{1}{\tau_{g}^{\prime}}\left(\epsilon g_{i}^{(1)}+\epsilon^{2} g_{i}^{(2)}\right)+\mathcal{O}\left(\epsilon^{3}\right),
\end{array}
$$

where $g_{i}(\boldsymbol{x}, t)$ has been subtracted and $\tau_{g}^{\prime}=\tau_{g} / \Delta t$. Matching 1st and 2nd orders terms in $\epsilon$ in this equation yields,

$$
\left(\partial_{t_{0}}+e_{i \alpha} \partial_{\alpha}\right) g_{i}^{(e q)}=-\frac{1}{\tau_{g}^{\prime}} g_{i}^{(1)},
$$




$$
\partial_{t_{1}} g_{i}^{(e q)}+\left(\partial_{t_{0}}+e_{i \alpha} \partial_{\alpha}\right) g_{i}^{(1)}+\frac{1}{2}\left(\partial_{t_{0}}+e_{i \alpha} \partial_{\alpha}\right)\left(\partial_{t_{0}}+e_{i \beta} \partial_{\beta}\right) g_{i}^{(e q)}=-\frac{1}{\tau_{g}^{\prime}} g_{i}^{(2)},
$$

respectively. Substituting Eq. A.5 into Eq. A.6 yields,

$$
\partial_{t_{1}} g_{i}^{(e q)}+\frac{2 \tau_{g}^{\prime}-1}{2 \tau_{g}^{\prime}}\left(\partial_{t_{0}}+e_{i \alpha} \partial_{\alpha}\right) g_{i}^{(1)}=-\frac{1}{\tau_{g}^{\prime}} g_{i}^{(2)} .
$$

According to the definitions of the zero-th and first moments of $g_{i}$ in Eqs. 1.25 and (1.26), we have for $k=1,2, \ldots$,

$$
\sum_{i=0}^{b} g_{i}^{(k)}=0 \quad \text { and } \quad \sum_{i=0}^{b} g_{i}^{(k)} e_{i \alpha}=0,
$$

and we can also calculate the third moment of $g_{i}$ as [?],

$$
\sum_{i=0}^{b} g_{i} e_{i \alpha} e_{i \beta} e_{i \gamma}=c_{s}^{2}\left(\delta_{\alpha \beta} u_{\gamma}^{*}+\delta_{\alpha \gamma} u_{\beta}^{*}+\delta_{\gamma \beta} u_{\alpha}^{*}\right)
$$

Now, taking the zero-th order moment of Eq. A.5 yields,

$$
\sum_{i=0}^{b}\left(\partial_{t_{0}}+e_{i \alpha} \partial_{\alpha}\right) g_{i}^{(e q)}=-\frac{1}{\tau_{g}^{\prime}} \sum_{i=0}^{b} g_{i}^{(1)}
$$

With the definition of $g_{i}^{(e q)}$ in Eq. 1.25, this yields,

$$
\partial_{\alpha} u_{\alpha}^{*}=0
$$

which is the incompressible mass conservation Eq. 1.14) (i.e., continuity equation).

The first-order moment of Eq. A.5 similarly yields,

$$
\sum_{i=0}^{b} e_{i \alpha}\left(\partial_{t_{0}}+e_{i \beta} \partial_{\beta}\right) g_{i}^{(e q)}=-\frac{1}{\tau_{g}^{\prime}} \sum_{i=0}^{b} e_{i \alpha} g_{i}^{(1)} .
$$

Using Eqs, (1.25), (1.26) and (1.27) in this equation, we obtain,

$$
\partial_{t_{0}} u_{\alpha}^{*}+\partial_{\beta}\left\{\left(c_{s}^{2}+\frac{k}{\rho} \frac{\partial \phi}{\partial x_{\gamma}} \frac{\partial \phi}{\partial x_{\gamma}}\right) \delta_{\alpha \beta}-\frac{k}{\rho} \frac{\partial \phi}{\partial x_{\alpha}} \frac{\partial \phi}{\partial x_{\beta}}+u_{\alpha}^{*} u_{\beta}^{*}\right\}=0 .
$$


Additionally, the first moments of Eq. A.7) reads,

$$
\partial_{t_{1}} u_{\alpha}^{*}+\frac{2 \tau_{g}^{\prime}-1}{2 \tau_{g}^{\prime}} \partial_{\beta} \Pi_{\alpha \beta}^{(1)}=0
$$

with the definition,

$$
\Pi_{\alpha \beta}^{(1)}=\sum_{i=0}^{b} g_{i}^{(1)} e_{i \alpha} e_{i \beta}
$$

By substituting $g_{i}^{(1)}$ from Eq. A.5 into Eq. A.15 we find,

$$
\begin{aligned}
\Pi_{\alpha \beta}^{(1)}=-\tau_{g}^{\prime} \sum_{i=0}^{b} e_{i \alpha} e_{i \beta}\left(\partial_{t_{0}}+e_{i \gamma} \partial_{\gamma}\right) g_{i}^{(e q)} & =-\tau_{g}^{\prime}\left\{\partial_{t_{0}} \sum_{i=0}^{b} e_{i \alpha} e_{i \beta} g_{i}^{(e q)}\right. \\
& \left.+\partial_{\gamma} \sum_{i=0}^{b} e_{i \alpha} e_{i \beta} e_{i \gamma} g_{i}^{(e q)}\right\}
\end{aligned}
$$

and, using Eqs. A.9 and 1.28,

$$
\begin{gathered}
\Pi_{\alpha \beta}^{(1)}=-\tau_{g}^{\prime}\left\{\partial_{t_{0}}\left(\left(c_{s}^{2}+\frac{k}{\rho} \frac{\partial \phi}{\partial x_{\gamma}} \frac{\partial \phi}{\partial x_{\gamma}}\right) \delta_{\alpha \beta}-\frac{k}{\rho} \frac{\partial \phi}{\partial x_{\alpha}} \frac{\partial \phi}{\partial x_{\beta}}+u_{\alpha}^{*} u_{\beta}^{*}\right)\right. \\
\left.+\partial_{\gamma} c_{s}^{2}\left(\delta_{\alpha \beta} u_{\gamma}^{*}+\delta_{\alpha \gamma} u_{\beta}^{*}+\delta_{\gamma \beta} u_{\alpha}^{*}\right)\right\} .
\end{gathered}
$$

Finally, substituting Eqs. (A.11) and (A.13), we have,

$$
\Pi_{\alpha \beta}^{(1)}=-\tau_{g}^{\prime}\left\{c_{s}^{2}\left(\partial_{\alpha} u_{\beta}^{*}+\partial_{\beta} u_{\alpha}^{*}\right)+\partial_{\gamma}\left(u_{\alpha}^{*} u_{\beta}^{*} u_{\gamma}^{*}\right)-\frac{k}{\rho} \partial_{t_{0}}\left(\frac{\partial \phi}{\partial x_{\alpha}} \frac{\partial \phi}{\partial x_{\beta}}\right)\right\} .
$$

With this definition, Eq. A.14 becomes,

$$
\begin{aligned}
\partial_{t_{1}} u_{\alpha}^{*} & -\partial_{\beta}\left\{c_{s}^{2}\left(\tau_{g}^{\prime}-\frac{1}{2}\right)\left(\partial_{\alpha} u_{\beta}^{*}+\partial_{\beta} u_{\alpha}^{*}\right)\right\}+\partial_{\beta}\left\{\left(\tau_{g}^{\prime}-\frac{1}{2}\right) \partial_{\gamma}\left(u_{\alpha}^{*} u_{\beta}^{*} u_{\gamma}^{*}\right)\right\} \\
& -\left\{\left(\tau_{g}^{\prime}-\frac{1}{2}\right) \frac{k}{\rho} \partial_{t_{0}}\left(\frac{\partial \phi}{\partial x_{\alpha}} \frac{\partial \phi}{\partial x_{\beta}}\right)\right\}=0 .
\end{aligned}
$$

The last term in this equation $\left(u_{\alpha}^{*} u_{\beta}^{*} u_{\gamma}^{*}\right)$ is $\mathcal{O}\left(\mathrm{Ma}^{3}\right)$, in Mach number Ma (with $M a \ll 1$ ), and hence can be neglected versus the other terms (Xiaoyi et al.1997). Thus,

$$
\partial_{t_{1}} u_{\alpha}^{*}-\partial_{\beta}\left\{c_{s}^{2}\left(\tau_{g}^{\prime}-\frac{1}{2}\right)\left(\partial_{\alpha} u_{\beta}^{*}+\partial_{\beta} u_{\alpha}^{*}\right)\right\}-\left\{\left(\tau_{g}^{\prime}-\frac{1}{2}\right) \frac{k}{\rho} \partial_{t_{0}}\left(\frac{\partial \phi}{\partial x_{\alpha}} \frac{\partial \phi}{\partial x_{\beta}}\right)\right\}=0 .
$$


To recover the NS momentum equation, we multiply Eq. A.20 by $\epsilon=\Delta t$ and add it to Eq. A.13, yielding,

$$
\begin{aligned}
\partial_{t} u_{\alpha}^{*} & +\partial_{\beta}\left\{\left(c_{s}^{2}+\frac{k}{\rho} \frac{\partial \phi}{\partial x_{\gamma}} \frac{\partial \phi}{\partial x_{\gamma}}\right) \delta_{\alpha \beta}-\frac{k}{\rho} \frac{\partial \phi}{\partial x_{\alpha}} \frac{\partial \phi}{\partial x_{\beta}}+u_{\alpha}^{*} u_{\beta}^{*}\right\} \\
& -\partial_{\beta}\left\{c_{s}^{2} \Delta t\left(\tau_{g}^{\prime}-\frac{1}{2}\right)\left(\partial_{\alpha} u_{\beta}^{*}+\partial_{\beta} u_{\alpha}^{*}\right)\right\} \\
& -\frac{1}{c_{s}^{2}}\left\{c_{s}^{2} \Delta t\left(\tau_{g}^{\prime}-\frac{1}{2}\right) \frac{k}{\rho} \partial_{t_{0}}\left(\frac{\partial \phi}{\partial x_{\alpha}} \frac{\partial \phi}{\partial x_{\beta}}\right)\right\}=0 .
\end{aligned}
$$

The last term can be neglected as it is $\mathcal{O}\left(1 / c_{s}^{2}\right)$ (with $c_{s} \gg 1$ ) compared to the previous terms, yielding,

$$
\begin{aligned}
\partial_{t} u_{\alpha}^{*}+\partial_{\beta}\left(u_{\alpha}^{*} u_{\beta}^{*}\right) & =\frac{k}{\rho} \partial_{\beta}\left\{\frac{\partial \phi}{\partial x_{\gamma}} \frac{\partial \phi}{\partial x_{\gamma}} \delta_{\alpha \beta}-\frac{\partial \phi}{\partial x_{\alpha}} \frac{\partial \phi}{\partial x_{\beta}}\right\} \\
& +\partial_{\beta}\left\{c_{s}^{2}\left(\tau_{g}-\frac{1}{2} \Delta t\right)\left(\partial_{\alpha} u_{\beta}^{*}+\partial_{\beta}^{*} u_{\alpha}^{*}\right)\right\}
\end{aligned}
$$

which, using continuity equation, is identical to Eq. (1.32), excluding the body forces, which are directly added to Eq. (1.34). 


\section{APPENDIX B}

\section{Chapman-Enskog expansion for Cahn-Hilliard equation}

By applying a procedure similar to that in Appendix A to Eq. (1.42), we obtain,

$$
\epsilon\left(\partial_{t_{0}}+e_{i \alpha} \partial_{\alpha}\right) f_{i}^{(e q)}=-\frac{1}{\tau_{f}^{\prime}} \epsilon f_{i}^{(1)},
$$

with $\tau_{f}^{\prime}=\tau_{f} / \Delta t$, and,

$$
\partial_{t_{1}} f_{i}^{(e q)}-\left(\tau_{f}^{\prime}-\frac{1}{2}\right)\left(\partial_{t_{0}}+e_{i \alpha} \partial_{\alpha}\right)\left(\partial_{t_{0}}+e_{i \beta} \partial_{\beta}\right) f_{i}^{(e q)}=-\frac{1}{\tau_{f}^{\prime}} f_{i}^{(2)},
$$

on the $\mathcal{O}(\epsilon)$ scale and the $\mathcal{O}\left(\epsilon^{2}\right)$ scale, respectively.

From the definitions of $f_{i}$ and $f_{i}^{(e q)}$ we find,

$$
\sum_{i=0}^{b} f_{i}^{(k)}=0 \quad \text { and } \quad \sum_{i=0}^{b} f_{i} e_{\alpha}^{(k)}=0 \quad(k=1,2, \ldots) .
$$

Taking the zeroth-order moments of Eqs. (B.1) and (B.2) and using Eqs. (1.43)(1.45) yields,

$$
\partial_{t_{0}} \phi+\partial_{\alpha}\left(\phi u_{\alpha}\right)=0
$$

and,

$$
\begin{aligned}
\partial_{t_{1}} \phi & -\left(\tau_{f}^{\prime}-\frac{1}{2}\right)\left\{\partial_{t_{0}} \partial_{t_{0}} \phi+\partial_{\alpha} \partial_{t_{0}}\left(\phi u_{\alpha}\right)\right\} \\
& -\left(\tau_{f}^{\prime}-\frac{1}{2}\right)\left\{\partial_{\beta} \partial_{t_{0}}\left(\phi u_{\beta}\right)+\partial_{\alpha} \partial_{\beta}\left(\Gamma \mu_{\phi} \delta_{\alpha \beta}+\phi u_{\alpha} u_{\beta}\right)\right\}=0 .
\end{aligned}
$$

From Eq. (B.4) we have,

$$
\partial_{t_{0}} \partial_{t_{0}} \phi+\partial_{\alpha} \partial_{t_{0}}\left(\phi u_{\alpha}\right)=\partial_{t_{0}}\left(\partial_{t_{0}} \phi+\partial_{\alpha}\left(\phi u_{\alpha}\right)\right)=0,
$$

and simplify Eq. (B.5) into,

$$
\partial_{t_{1}} \phi=\left(\tau_{f}^{\prime}-\frac{1}{2}\right)\left\{\partial_{\beta} \partial_{t_{0}}\left(\phi u_{\beta}\right)+\partial_{\alpha} \partial_{\beta}\left(\Gamma \mu_{\phi} \delta_{\alpha \beta}+\phi u_{\alpha} u_{\beta}\right)\right\},
$$


or

$$
\partial_{t_{1}} \phi=\left(\tau_{f}^{\prime}-\frac{1}{2}\right) \partial_{\beta}\left\{\partial_{t_{0}}\left(\phi u_{\beta}\right)+\partial_{\alpha}\left(\phi u_{\alpha} u_{\beta}\right)\right\}+\left(\tau_{f}^{\prime}-\frac{1}{2}\right) \partial_{\alpha} \partial_{\alpha} \Gamma \mu_{\phi} .
$$

Multiplying Eq. (B.8) by $\Delta t$ and adding it to Eq. (B.4), we get,

$$
\begin{aligned}
\partial_{t} \phi+\partial_{\alpha}\left(\phi u_{\alpha}\right)= & \Delta t\left(\tau_{f}^{\prime}-\frac{1}{2}\right) \Gamma \nabla^{2} \mu_{\phi} \\
& +\Delta t\left(\tau_{f}^{\prime}-\frac{1}{2}\right) \partial_{\beta}\left\{\partial_{t_{0}}\left(\phi u_{\beta}\right)+\partial_{\alpha}\left(\phi u_{\alpha} u_{\beta}\right)\right\} .
\end{aligned}
$$

For low Mach number flows, the last term can be neglected, since it has a small magnitude and is multiplied by the small parameter $\Delta t$, so that we finally get,

$$
\partial_{t} \phi+\partial_{\alpha}\left(\phi u_{\alpha}\right)=M \nabla^{2} \mu_{\phi}
$$

which corresponds to the Cahn-Hilliard equation with diffusivity coefficient (Mobility) defined as,

$$
M=\left(\tau_{f}^{\prime}-\frac{1}{2}\right) \Gamma \Delta t
$$




\section{APPENDIX C}

\section{Chapman-Enskog expansion for pressure Poisson equation}

Here, the Chapman-Enskog multiscale expansion procedure is applied to the Lattice Boltzmann equation for solving the pressure Poisson equation (1.38), which reads,

$$
h_{i}^{n}\left(\boldsymbol{x}+\boldsymbol{e}_{i} \Delta t, t+\Delta t\right)=h_{i}^{n}(\boldsymbol{x}, t)-\frac{1}{\tau_{h}^{\prime}}\left(h_{i}^{n}-h_{i}^{(e q, n)}\right)-\Delta t w_{i}\left(\nabla \cdot \boldsymbol{u}^{*}(t)\right),
$$

where $\tau_{h}^{\prime}=\tau_{h} / \Delta t$. Again we apply a Taylor series expansion to the left hand side, yielding,

$$
\begin{aligned}
h_{i}^{n}\left(\boldsymbol{x}+\boldsymbol{e}_{i} \Delta t, t+\Delta t\right) & =h_{i}^{n}(\boldsymbol{x}, t)+\epsilon\left(\partial_{t}+e_{i \alpha} \partial_{\alpha}\right) h_{i}^{n}(\boldsymbol{x}, t) \\
& +\frac{1}{2} \epsilon^{2}\left(\partial_{t}+e_{i \alpha} \partial_{\alpha}\right)\left(\partial_{t}+e_{i \beta} \partial_{\beta}\right) h_{i}^{n}(\boldsymbol{x}, t)+\mathcal{O}\left(\epsilon^{3}\right),
\end{aligned}
$$

and expand the particle distribution function with $\epsilon=\Delta t$ being small compared to the macroscopic time scales,

$$
\begin{aligned}
& h_{i}^{n}=h_{i}^{(e q, n)}+\epsilon h_{i}^{(1)}+\epsilon^{2} h_{i}^{(2)}+\mathcal{O}\left(\epsilon^{3}\right), \\
& \partial_{t}=\partial_{t_{0}}+\epsilon \partial_{t_{1}}+\mathcal{O}\left(\epsilon^{2}\right) .
\end{aligned}
$$

Substituting these equations into Eq. (C.1) and using Eq. (C.2), we find,

$$
\begin{aligned}
& \epsilon\left(\partial_{t_{0}}+e_{i \alpha} \partial_{\alpha}\right) h_{i}^{(e q, n)}+\epsilon^{2}\left\{\partial_{t_{1}} h_{i}^{(e q, n)}+\left(\partial_{t_{0}}+e_{i \alpha} \partial_{\alpha}\right) h_{i}^{(1)}+\frac{1}{2}\left(\partial_{t_{0}}+e_{i \alpha} \partial_{\alpha}\right)\right. \\
& \left.\left(\partial_{t_{0}}+e_{i \beta} \partial_{\beta}\right) h_{i}^{(e q, n)}\right\}=-\frac{1}{\tau_{h}^{\prime}}\left(\epsilon h_{i}^{(1)}+\epsilon^{2} h_{i}^{(2)}\right)-\epsilon w_{i} \nabla \cdot \boldsymbol{u}^{*}+\mathcal{O}\left(\epsilon^{3}\right),
\end{aligned}
$$

and, after matching different orders of $\epsilon$, we get,

$$
\left(\partial_{t_{0}}+e_{i \alpha} \partial_{\alpha}\right) h_{i}^{(e q, n)}=-\frac{1}{\tau_{h}^{\prime}} h_{i}^{(1)}-w_{i} \nabla \cdot \boldsymbol{u}^{*}
$$

and,

$$
\partial_{t_{1}} h_{i}^{(e q, n)}+\left(\partial_{t_{0}}+e_{i \alpha} \partial_{\alpha}\right) h_{i}^{(1)}+\frac{1}{2}\left(\partial_{t_{0}}+e_{i \alpha} \partial_{\alpha}\right)\left(\partial_{t_{0}}+e_{i \beta} \partial_{\beta}\right) h_{i}^{(e q, n)}=-\frac{1}{\tau_{h}^{\prime}} h_{i}^{(2)},
$$


for the $\mathcal{O}(\epsilon)$ and $\mathcal{O}\left(\epsilon^{2}\right)$ terms, respectively. Substituting (C.6) into (C.7), we obtain,

$$
\partial_{t_{1}} h_{i}^{(e q, n)}+\frac{2 \tau_{h}^{\prime}-1}{2 \tau_{h}^{\prime}}\left(\partial_{t_{0}}+e_{i \alpha} \partial_{\alpha}\right) h_{i}^{(1)}-\frac{w_{i}}{2}\left(\partial_{t_{0}}+e_{i \alpha} \partial_{\alpha}\right) \nabla \cdot \boldsymbol{u}^{*}=-\frac{1}{\tau_{h}^{\prime}} h_{i}^{(2)} .
$$

From the definitions of $h_{i}^{n}$ and $h_{i}^{(e q, n)}$ we find,

$$
\sum_{i=0}^{b} h_{i}^{(k)}=0 \quad(k=1,2, \ldots) ;
$$

we also have from Eq. (1.39), for the zero-th and 2nd-moments (1st moment is zero),

$$
\begin{aligned}
& \sum_{i=0}^{b} h_{i}^{n}=\frac{p^{n+1}}{\rho_{0} c^{2}}, \\
& \sum_{i=0}^{b} h_{i}^{n} e_{i \alpha} e_{i \beta}=c_{s}^{2} \delta_{\alpha \beta} \frac{p^{n+1}}{\rho_{0} c^{2}} .
\end{aligned}
$$

The zeroth-order moment of Eq. (C.6) leads to,

$$
\partial_{t_{0}}\left(\frac{p}{\rho_{0} c^{2}}\right)=-\nabla \cdot \boldsymbol{u}^{*}
$$

and, given $\sum w_{i}=1$ and $\sum w_{i} e_{i \alpha}=0$, the zeroth-order moment of Eq. (C.8) yields,

$$
\partial_{t_{1}}\left(\frac{p}{\rho_{0} c^{2}}\right)+\Pi^{(1)}-\frac{1}{2} \partial_{t_{0}}\left(\nabla \cdot \boldsymbol{u}^{*}\right)=0,
$$

where the scalar $\Pi^{(1)}$ is defined as,

$$
\Pi^{(1)}=\frac{2 \tau_{h}^{\prime}-1}{2 \tau_{h}^{\prime}} \sum_{i=0}^{b}\left(\partial_{t_{0}}+e_{i \alpha} \partial_{\alpha}\right) h_{i}^{(1)} .
$$

by using (C.9) we have,

$$
\Pi^{(1)}=\frac{2 \tau_{h}^{\prime}-1}{2 \tau_{h}^{\prime}} \partial_{\alpha} \sum_{i=0}^{b} e_{i \alpha} h_{i}^{(1)} .
$$

Fom Eq. (C.6) we have,

$$
h_{i}^{(1)}=-\tau_{h}^{\prime}\left\{\left(\partial_{t_{0}}+e_{i \alpha} \partial_{\alpha}\right) h_{i}^{(e q, n)}+w_{i} \nabla \cdot \boldsymbol{u}^{*}\right\},
$$


then,

$$
\Pi^{(1)}=\frac{1-2 \tau_{h}^{\prime}}{2} \sum_{i=0}^{b}\left(e_{i \alpha} \partial_{\alpha}\right)\left\{\left(\partial_{t_{0}}+e_{i \beta} \partial_{\beta}\right) h_{i}^{(e q, n)}+w_{i} \nabla \cdot \boldsymbol{u}^{*}\right\} .
$$

Therefore, using Eqs. C.10, given $\sum e_{i \alpha} h_{i}^{n}=0$,

$$
\Pi^{(1)}=\partial_{\alpha}\left\{\frac{1-2 \tau_{h}^{\prime}}{2 \rho_{0} c^{2}}\left(c_{s}^{2} \partial_{\alpha} p^{n+1}\right)\right\}
$$

Replacing $\Pi^{(1)}$ into Eq. C.12 we have,

$$
\partial_{t_{1}}\left(\frac{p}{\rho_{0} c^{2}}\right)+\partial_{\alpha}\left(\frac{1-2 \tau_{h}^{\prime}}{2 \rho_{0} c^{2}}\left(c_{s}^{2} \partial_{\alpha} p^{n+1}\right)\right)-\frac{1}{2} \partial_{t_{0}}\left(\nabla \cdot \boldsymbol{u}^{*}\right)=0
$$

Equation (C.18) is multiplied by $\epsilon=\Delta t$ and added to Eq. C.11 one gets,

$$
\partial_{t}\left(\frac{p^{n+1}}{\rho_{0} c^{2}}\right)+\partial_{\alpha}\left\{\frac{1-2 \tau_{h}^{\prime}}{2 \rho_{0} c^{2}} \Delta t c_{s}^{2} \partial_{\alpha} p^{n+1}\right\}=-\nabla \cdot \boldsymbol{u}^{*}
$$

where we neglected $\frac{1}{2} \partial_{t_{0}}\left(\nabla \cdot \boldsymbol{u}^{*}\right)$, as compared to larger zero-th order terms.

Now, if $\partial_{t} p^{n+1} \rightarrow 0$, then Eq. C.19 becomes the diffusive Poisson Eq. (1.37) provided,

$$
\frac{2 \tau_{h}^{\prime}-1}{2 \rho_{0} c^{2}} \Delta t c_{s}^{2}=\frac{\Delta t}{\rho}
$$

or,

$$
\tau_{h}=\left(\frac{1}{2}+\frac{c^{2} \rho_{0}}{c_{s}^{2} \rho}\right) \Delta t
$$




\section{APPENDIX D}

\section{Chapman-Enskog expansion to LB equations for solving multiphase} flow with high density ratio and high Re number flows to retrieve Navier Stokes equation

Here we apply Chapman-Enskog expansion to verify that the mesoscopic evolution Eq. 2.4 , with the equilibrium distribution functions defined in Eqs. 22.5. 2.8), converges to the macroscopic "pressureless" NS equations of motion Eq. 2.3.

It should be mentioned that the below properties of the D3Q19 lattice scheme have been used in deriving the equations in this section,

$$
\begin{aligned}
& \sum_{i=0}^{b} w_{i}=1 \\
& \sum_{i=0}^{b} w_{i} e_{i \alpha}=0 \\
& \sum_{i=0}^{b} w_{i} e_{i \alpha} e_{i \beta}=c_{s}^{2} \delta_{\alpha \beta} \\
& \sum_{i=0}^{b} w_{i} e_{i \alpha} e_{i \beta} e_{i \gamma}=0 \\
& \sum_{i=0}^{b} w_{i} e_{i \alpha} e_{i \beta} e_{i \gamma} e_{i \zeta}=c_{s}^{4}\left(\delta_{\alpha \beta} \delta_{\gamma \zeta}+\delta_{\alpha \gamma} \delta_{\beta \zeta}+\delta_{\alpha \zeta} \delta_{\gamma \beta}\right) \\
& \sum_{i=0}^{b} w_{i} e_{i \alpha} e_{i \beta} e_{i \gamma} e_{i \zeta} e_{i \gamma}=0
\end{aligned}
$$


The zeroth-, first-, second- and third-order moments of equilibrium distribution functions Eq. (2.5) are calculated as,

$$
\begin{aligned}
& \sum_{i=0}^{b} g_{i}^{(e q)}=\sum_{i=0}^{b} g_{i}=1, \\
& \sum_{i=0}^{b} g_{i}^{(e q)} e_{i \alpha}=\sum_{i=0}^{b} g_{i} e_{i \alpha}=u_{\alpha}^{*}, \\
& \sum_{i=0}^{b} g_{i}^{(e q)} e_{i \alpha} e_{i \beta}=\Pi_{\alpha \beta}^{(0)}=\left(c_{s}^{2}+\frac{k}{\rho} \frac{\partial \phi}{\partial x_{\gamma}} \frac{\partial \phi}{\partial x_{\gamma}}\right) \delta_{\alpha \beta}-\frac{k}{\rho} \frac{\partial \phi}{\partial x_{\alpha}} \frac{\partial \phi}{\partial x_{\beta}} \\
& +u_{\alpha}^{*} u_{\beta}^{*}+c_{s}^{2}\left(\tau_{g}^{*}-\frac{1}{2}\right)\left(\frac{\partial u_{\alpha}}{\partial x_{\beta}}+\frac{\partial u_{\beta}}{\partial x_{\alpha}}\right) \Delta t \\
& \sum_{i=0}^{b} g_{i} e_{i \alpha} e_{i \beta} e_{i \gamma}=c_{s}^{2}\left(\delta_{\alpha \beta} u_{\gamma}^{*}+\delta_{\alpha \gamma} u_{\beta}^{*}+\delta_{\gamma \beta} u_{\alpha}^{*}\right) .
\end{aligned}
$$

The Taylor series expansion in space and time of the left hand side of LB Eq. (2.4) yields,

$$
\begin{aligned}
g_{i}\left(\boldsymbol{x}+e_{i \alpha} \Delta t, t+\Delta t\right) & =g_{i}(\boldsymbol{x}, t)+\epsilon\left(\partial_{t}+e_{i \alpha} \partial_{\alpha}\right) g_{i}(\boldsymbol{x}, t) \\
& +\frac{1}{2} \epsilon^{2}\left(\partial_{t}+e_{i \alpha} \partial_{\alpha}\right)\left(\partial_{t}+e_{i \beta} \partial_{\beta}\right) g_{i}(\boldsymbol{x}, t)+\mathcal{O}\left(\epsilon^{3}\right)
\end{aligned}
$$

with the expansion parameter $\epsilon=\Delta t$ being small compared to the macroscopic time scales. Then, we expand the particle distribution function $g_{i}(\boldsymbol{x}, t)$ as,

$$
g_{i}=g_{i}^{(e q)}+\epsilon g_{i}^{(1)}+\mathcal{O}\left(\epsilon^{2}\right)
$$

with the following multiple-scale expansion for the time derivative,

$$
\partial_{t}=\partial_{t_{0}}+\epsilon \partial_{t_{1}}+\mathcal{O}\left(\epsilon^{2}\right)
$$

where $t_{0}$ denotes the "slow" (macroscopic) time and $t_{1}$ is the "fast" time.

Substituting the latter two equations into Eq (D.11) and sorting out terms in 
orders of $\epsilon$, we obtain,

$$
\begin{array}{r}
\epsilon\left(\partial_{t_{0}}+e_{i \alpha} \partial_{\alpha}\right) g_{i}^{(e q)}+\epsilon^{2}\left\{\partial_{t_{1}} g_{i}^{(e q)}+\left(\partial_{t_{0}}+e_{i \alpha} \partial_{\alpha}\right) g_{i}^{(1)}+\frac{1}{2}\left(\partial_{t_{0}}+e_{i \alpha} \partial_{\alpha}\right)\right. \\
\left.\left(\partial_{t_{0}}+e_{i \beta} \partial_{\beta}\right) g_{i}^{(e q)}\right\}+\mathcal{O}\left(\epsilon^{3}\right)=-\frac{1}{\tau_{g}^{*}}\left(\epsilon g_{i}^{(1)}+\epsilon^{2} g_{i}^{(2)}\right)+\mathcal{O}\left(\epsilon^{3}\right)
\end{array}
$$

where $g_{i}(\boldsymbol{x}, t)$ has been subtracted and $\tau_{g}^{*}=\tau_{g} / \Delta t$. Matching terms of 1 st and 2nd orders in $\epsilon$ in this equation yields,

$$
\begin{gathered}
\left(\partial_{t_{0}}+e_{i \alpha} \partial_{\alpha}\right) g_{i}^{(e q)}=-\frac{1}{\tau_{g}^{*}} g_{i}^{(1)} \\
\partial_{t_{1}} g_{i}^{(e q)}+\left(\partial_{t_{0}}+e_{i \alpha} \partial_{\alpha}\right) g_{i}^{(1)}+\frac{1}{2}\left(\partial_{t_{0}}+e_{i \alpha} \partial_{\alpha}\right)\left(\partial_{t_{0}}+e_{i \beta} \partial_{\beta}\right) g_{i}^{(e q)}=-\frac{1}{\tau_{g}^{*}} g_{i}^{(2)} .
\end{gathered}
$$

respectively. Substituting Eq. (D.15) into Eq. (D.16) yields,

$$
\partial_{t_{1}} g_{i}^{(e q)}+\frac{2 \tau_{g}^{*}-1}{2 \tau_{g}^{*}}\left(\partial_{t_{0}}+e_{i \alpha} \partial_{\alpha}\right) g_{i}^{(1)}=-\frac{1}{\tau_{g}^{*}} g_{i}^{(2)}
$$

According to the definitions of the zeroth- and first-order moments of $g_{i}$ in Eqs. (D.7) and (D.8), we get for $k=1,2, \ldots$,

$$
\sum_{i=0}^{b} g_{i}^{(k)}=0 \quad \text { and } \quad \sum_{i=0}^{b} g_{i}^{(k)} e_{i \alpha}=0,
$$

Now taking zero-th order moment of Eq. (D.15,

$$
\sum_{i=0}^{b}\left(\partial_{t_{0}}+e_{i \alpha} \partial_{\alpha}\right) g_{i}^{(e q)}=-\frac{1}{\tau_{g}^{*}} \sum_{i=0}^{b} g_{i}^{(1)}
$$

with the definition of $g_{i}^{(e q)}$ in Eq. (D.7), yields,

$$
\partial_{\alpha} u_{\alpha}^{*}=0
$$

which is the incompressible mass conservation Eq. (2.2) (i.e., continuity equation). Taking the first-order moment of Eq. (D.15) yields,

$$
\sum_{i=0}^{b} e_{i \alpha}\left(\partial_{t_{0}}+e_{i \beta} \partial_{\beta}\right) g_{i}^{(e q)}=-\frac{1}{\tau_{g}^{*}} \sum_{i=0}^{b} e_{i \alpha} g_{i}^{(1)}
$$




$$
\partial_{t_{0}} \sum_{i=0}^{b} e_{i \alpha} g_{i}^{(e q)}+\partial_{\beta} \Pi_{\alpha \beta}^{(0)}=0
$$

Using Eqs, (D.7), (D.8) and (D.9) we obtain,

$$
\begin{array}{r}
\partial_{t_{0}} u_{\alpha}^{*}+\partial_{\beta}\left\{\left(c_{s}^{2}+\frac{k}{\rho} \frac{\partial \phi}{\partial x_{\gamma}} \frac{\partial \phi}{\partial x_{\gamma}}\right) \delta_{\alpha \beta}-\frac{k}{\rho} \frac{\partial \phi}{\partial x_{\alpha}} \frac{\partial \phi}{\partial x_{\beta}}\right. \\
\left.+u_{\alpha}^{*} u_{\beta}^{*}+c_{s}^{2}\left(\tau_{g}^{*}-\frac{1}{2}\right)\left(\frac{\partial u_{\alpha}}{\partial x_{\beta}}+\frac{\partial u_{\beta}}{\partial x_{\alpha}}\right) \Delta t\right\}=0
\end{array}
$$

Additionally, the first-order moment of Eq. (D.17) reads,

$$
\partial_{t_{1}} u_{\alpha}^{*}+\frac{2 \tau_{g}^{*}-1}{2 \tau_{g}^{*}} \partial_{\beta} \Pi_{\alpha \beta}^{(1)}=0,
$$

with the definition,

$$
\Pi_{\alpha \beta}^{(1)}=\sum_{i=0}^{b} g_{i}^{(1)} e_{i \alpha} e_{i \beta}
$$

Substituting $g_{i}^{(1)}$ from Eq. D.15 into Eq. D.25, and using Eqs. D.10 and (D.9), we find,

$$
\begin{aligned}
& \Pi_{\alpha \beta}^{(1)}=-\tau_{g}^{*} \sum_{i=0}^{b} e_{i \alpha} e_{i \beta}\left(\partial_{t_{0}}+e_{i \gamma} \partial_{\gamma}\right) g_{i}^{(e q)}=-\tau_{g}^{*}\left\{\partial_{t_{0}} \sum_{i=0}^{b} e_{i \alpha} e_{i \beta} g_{i}^{(e q)}\right. \\
&\left.+\partial_{\gamma} \sum_{i=0}^{b} e_{i \alpha} e_{i \beta} e_{i \gamma} g_{i}^{(e q)}\right\} \\
& \Pi_{\alpha \beta}^{(1)}=-\tau_{g}^{*}\left\{\partial_{t_{0}} \Pi_{\alpha \beta}^{(0)}+\partial_{\gamma} c_{s}^{2}\left(\delta_{\alpha \beta} u_{\gamma}^{*}+\delta_{\alpha \gamma} u_{\beta}^{*}+\delta_{\gamma \beta} u_{\alpha}^{*}\right)\right\}
\end{aligned}
$$

then,

$$
\begin{aligned}
\Pi_{\alpha \beta}^{(1)}= & -\tau_{g}^{*}\left\{\partial _ { t _ { 0 } } \left(\left(c_{s}^{2}+\frac{k}{\rho} \frac{\partial \phi}{\partial x_{\gamma}} \frac{\partial \phi}{\partial x_{\gamma}}\right) \delta_{\alpha \beta}-\frac{k}{\rho} \frac{\partial \phi}{\partial x_{\alpha}} \frac{\partial \phi}{\partial x_{\beta}}\right.\right. \\
& \left.+u_{\alpha}^{*} u_{\beta}^{*}+c_{s}^{2}\left(\tau_{g}^{*}-\frac{1}{2}\right)\left(\frac{\partial u_{\alpha}}{\partial x_{\beta}}+\frac{\partial u_{\beta}}{\partial x_{\alpha}}\right) \Delta t\right) \\
& \left.+\partial_{\gamma} c_{s}^{2}\left(\delta_{\alpha \beta} u_{\gamma}^{*}+\delta_{\alpha \gamma} u_{\beta}^{*}+\delta_{\gamma \beta} u_{\alpha}^{*}\right)\right\}
\end{aligned}
$$


which can be simplified to,

$$
\begin{gathered}
\Pi_{\alpha \beta}^{(1)}=-\tau_{g}^{*}\left\{\partial _ { t _ { 0 } } \left(\frac{k}{\rho} \frac{\partial \phi}{\partial x_{\gamma}} \frac{\partial \phi}{\partial x_{\gamma}} \delta_{\alpha \beta}-\frac{k}{\rho} \frac{\partial \phi}{\partial x_{\alpha}} \frac{\partial \phi}{\partial x_{\beta}}+u_{\alpha}^{*} u_{\beta}^{*}\right.\right. \\
\left.\left.+c_{s}^{2}\left(\tau_{g}^{*}-\frac{1}{2}\right)\left(\frac{\partial u_{\alpha}}{\partial x_{\beta}}+\frac{\partial u_{\beta}}{\partial x_{\alpha}}\right) \Delta t\right)+c_{s}^{2}\left(\partial_{\alpha} u_{\beta}^{*}+\partial_{\beta} u_{\alpha}^{*}\right)\right\}
\end{gathered}
$$

where continuity equation Eq. (2.2) has been used in the last term. Using chain rule in the term $\partial t_{0}\left(u_{\alpha}^{*} u_{\beta}^{*}\right)$ yields,

$$
\partial t_{0}\left(u_{\alpha}^{*} u_{\beta}^{*}\right)=u_{\alpha}^{*} \partial t_{0}\left(u_{\beta}^{*}\right)+u_{\beta}^{*} \partial t_{0}\left(u_{\alpha}^{*}\right)
$$

with, from Eq. (D.22),

$$
\begin{gathered}
\partial t_{0}\left(u_{\beta}\right)=-\partial_{\gamma}\left(\Pi_{\beta \gamma}^{0}\right) \\
\partial t_{0}\left(u_{\alpha}\right)=-\partial_{\gamma}\left(\Pi_{\alpha \gamma}^{0}\right)
\end{gathered}
$$

yielding after substitution,

$$
\partial t_{0}\left(u_{\alpha}^{*} u_{\beta}^{*}\right)=-u_{\alpha}^{*} \partial_{\gamma}\left(\Pi_{\beta \gamma}^{0}\right)-u_{\beta}^{*} \partial_{\gamma}\left(\Pi_{\alpha \gamma}^{0}\right)
$$

Therefore,

$$
\begin{aligned}
& \partial t_{0}\left(u_{\alpha}^{*} u_{\beta}^{*}\right)=-u_{\alpha}^{*} \partial_{\gamma}\left\{\left(c_{s}^{2}+\frac{k}{\rho} \frac{\partial \phi}{\partial x_{\zeta}} \frac{\partial \phi}{\partial x_{\zeta}}\right) \delta_{\beta \gamma}\right. \\
& \left.-\frac{k}{\rho} \frac{\partial \phi}{\partial x_{\beta}} \frac{\partial \phi}{\partial x_{\gamma}}+u_{\beta}^{*} u_{\gamma}^{*}+c_{s}^{2}\left(\tau_{g}^{*}-\frac{1}{2}\right) \Delta t\left(\frac{\partial u_{\beta}}{\partial x_{\gamma}}+\frac{\partial u_{\gamma}}{\partial x_{\beta}}\right)\right\} \\
& -u_{\beta}^{*} \partial_{\gamma}\left\{\left(c_{s}^{2}+\frac{k}{\rho} \frac{\partial \phi}{\partial x_{\zeta}} \frac{\partial \phi}{\partial x_{\zeta}}\right) \delta_{\alpha \gamma}-\frac{k}{\rho} \frac{\partial \phi}{\partial x_{\alpha}} \frac{\partial \phi}{\partial x_{\gamma}}\right. \\
& \left.+u_{\alpha}^{*} u_{\gamma}^{*}+c_{s}^{2}\left(\tau_{g}^{*}-\frac{1}{2}\right) \Delta t\left(\frac{\partial u_{\alpha}}{\partial x_{\gamma}}+\frac{\partial u_{\gamma}}{\partial x_{\alpha}}\right)\right\}
\end{aligned}
$$


then,

$$
\begin{aligned}
& \partial t_{0}\left(u_{\alpha}^{*} u_{\beta}^{*}\right)=-u_{\alpha}^{*} \partial_{\gamma}\left(u_{\beta}^{*} u_{\gamma}^{*}\right)-u_{\beta}^{*} \partial_{\gamma}\left(u_{\alpha}^{*} u_{\gamma}^{*}\right) \\
& -u_{\alpha}^{*} \partial_{\gamma}\left\{\left(c_{s}^{2}+\frac{k}{\rho} \frac{\partial \phi}{\partial x_{\zeta}} \frac{\partial \phi}{\partial x_{\zeta}}\right) \delta_{\beta \gamma}\right. \\
& \left.-\frac{k}{\rho} \frac{\partial \phi}{\partial x_{\beta}} \frac{\partial \phi}{\partial x_{\gamma}}+c_{s}^{2}\left(\tau_{g}^{*}-\frac{1}{2}\right) \Delta t\left(\frac{\partial u_{\beta}}{\partial x_{\gamma}}+\frac{\partial u_{\gamma}}{\partial x_{\beta}}\right)\right\} \\
& -u_{\beta}^{*} \partial_{\gamma}\left\{\left(c_{s}^{2}+\frac{k}{\rho} \frac{\partial \phi}{\partial x_{\zeta}} \frac{\partial \phi}{\partial x_{\zeta}}\right) \delta_{\alpha \gamma}\right. \\
& \left.-\frac{k}{\rho} \frac{\partial \phi}{\partial x_{\alpha}} \frac{\partial \phi}{\partial x_{\gamma}}+c_{s}^{2}\left(\tau_{g}^{*}-\frac{1}{2}\right) \Delta t\left(\frac{\partial u_{\alpha}}{\partial x_{\gamma}}+\frac{\partial u_{\gamma}}{\partial x_{\alpha}}\right)\right\}
\end{aligned}
$$

The first term in the above equation can be replaced by $-\partial_{\gamma}\left(u_{\beta}^{*} u_{\alpha}^{*} u_{\gamma}^{*}\right)$. Now, replacing $\partial t_{0}\left(u_{\alpha}^{*} u_{\beta}^{*}\right)$ from the above equation into Eq. D.36 results in,

$$
\begin{gathered}
\Pi_{\alpha \beta}^{(1)}=-\tau_{g}^{*}\left\{\partial_{t_{0}}\left(\frac{k}{\rho} \frac{\partial \phi}{\partial x_{\gamma}} \frac{\partial \phi}{\partial x_{\gamma}} \delta_{\alpha \beta}-\frac{k}{\rho} \frac{\partial \phi}{\partial x_{\alpha}} \frac{\partial \phi}{\partial x_{\beta}}+c_{s}^{2}\left(\tau_{g}^{*}-\frac{1}{2}\right) \Delta t\left(\frac{\partial u_{\alpha}}{\partial x_{\beta}}+\frac{\partial u_{\beta}}{\partial x_{\alpha}}\right)\right)\right. \\
-\partial_{\gamma}\left(u_{\beta}^{*} u_{\alpha}^{*} u_{\gamma}^{*}\right) \\
-u_{\alpha}^{*} \partial_{\gamma}\left(\left(c_{s}^{2}+\frac{k}{\rho} \frac{\partial \phi}{\partial x_{\zeta}} \frac{\partial \phi}{\partial x_{\zeta}}\right) \delta_{\beta \gamma}-\frac{k}{\rho} \frac{\partial \phi}{\partial x_{\beta}} \frac{\partial \phi}{\partial x_{\gamma}}+c_{s}^{2}\left(\tau_{g}^{*}-\frac{1}{2}\right) \Delta t\left(\frac{\partial u_{\beta}}{\partial x_{\gamma}}+\frac{\partial u_{\gamma}}{\partial x_{\beta}}\right)\right) \\
-u_{\beta}^{*} \partial_{\gamma}\left(\left(c_{s}^{2}+\frac{k}{\rho} \frac{\partial \phi}{\partial x_{\zeta}} \frac{\partial \phi}{\partial x_{\zeta}}\right) \delta_{\alpha \gamma}-\frac{k}{\rho} \frac{\partial \phi}{\partial x_{\alpha}} \frac{\partial \phi}{\partial x_{\gamma}}+c_{s}^{2}\left(\tau_{g}^{*}-\frac{1}{2}\right) \Delta t\left(\frac{\partial u_{\alpha}}{\partial x_{\gamma}}+\frac{\partial u_{\gamma}}{\partial x_{\alpha}}\right)\right) \\
\left.+c_{s}^{2}\left(\partial_{\alpha} u_{\beta}^{*}+\partial_{\beta} u_{\alpha}^{*}\right)\right\}
\end{gathered}
$$


With this definition, Eq. (D.24) becomes,

$$
\begin{aligned}
\partial_{t_{1}} u_{\alpha}^{*} & -\partial_{\beta}\left\{c_{s}^{2}\left(\tau_{g}^{*}-\frac{1}{2}\right)\left(\partial_{\alpha} u_{\beta}^{*}+\partial_{\beta} u_{\alpha}^{*}\right)\right\} \\
& +\partial_{\beta}\left\{\left(\tau_{g}^{*}-\frac{1}{2}\right) \partial_{\gamma}\left(u_{\alpha}^{*} u_{\beta}^{*} u_{\gamma}^{*}\right)\right\} \\
& -\partial_{\alpha} \partial_{t_{0}}\left\{\left(\tau_{g}^{*}-\frac{1}{2}\right) \frac{k}{\rho}\left(\frac{\partial \phi}{\partial x_{\gamma}} \frac{\partial \phi}{\partial x_{\gamma}}\right)\right\} \\
& +\partial_{\beta} \partial_{t_{0}}\left\{\left(\tau_{g}^{*}-\frac{1}{2}\right) \frac{k}{\rho}\left(\frac{\partial \phi}{\partial x_{\alpha}} \frac{\partial \phi}{\partial x_{\beta}}\right)\right\} \\
& +\partial_{\beta} \partial_{t_{0}}\left\{c_{s}^{2}\left(\tau_{g}^{*}-\frac{1}{2}\right)^{2} \Delta t\left(\frac{\partial u_{\alpha}}{\partial x_{\beta}}+\frac{\partial u_{\beta}}{\partial x_{\alpha}}\right)\right\} \\
& +u_{\alpha}^{*} \partial_{\beta}^{2}\left\{\left(\tau_{g}^{*}-\frac{1}{2}\right) \frac{k}{\rho}\left(\frac{\partial \phi}{\partial x_{\gamma}} \frac{\partial \phi}{\partial x_{\gamma}}\right)\right\} \\
& -u_{\alpha}^{*} \partial_{\beta} \partial_{\gamma}\left\{\left(\tau_{g}^{*}-\frac{1}{2}\right) \frac{k}{\rho}\left(\frac{\partial \phi}{\partial x_{\beta}} \frac{\partial \phi}{\partial x_{\gamma}}\right)\right\} \\
& +u_{\alpha}^{*} \partial_{\beta} \partial_{\gamma}\left\{c_{s}^{2}\left(\tau_{g}^{*}-\frac{1}{2}\right)^{2} \Delta t\left(\frac{\partial u_{\gamma}}{\partial x_{\beta}}+\frac{\partial u_{\beta}}{\partial x_{\gamma}}\right)\right\} \\
+ & u_{\beta}^{*} \partial_{\beta} \partial_{\alpha}\left\{\left(\tau_{g}^{*}-\frac{1}{2}\right) \frac{k}{\rho}\left(\frac{\partial \phi}{\partial x_{\gamma}} \frac{\partial \phi}{\partial x_{\gamma}}\right)\right\} \\
& +u_{\beta}^{*} \partial_{\beta} \partial_{\gamma}\left\{\left(\tau_{g}^{*}-\frac{1}{2}\right) \frac{k}{\rho}\left(\frac{\partial \phi}{\partial x_{\alpha}} \frac{\partial \phi}{\partial x_{\gamma}}\right)\right\} \\
& \left.+\partial_{\beta} \partial_{\gamma}\left\{c_{s}^{2}\left(\tau_{g}^{*}-\frac{\partial}{2}\right)^{2} \frac{\partial u_{\gamma}}{\partial x_{\alpha}}+\frac{\partial u_{\alpha}}{\partial x_{\gamma}}\right)\right\}=0
\end{aligned}
$$

To recover the pressureless NS momentum equation, we multiply Eq. (D.37) by 
$\epsilon=\Delta t$ and add it to Eq. (D.23), yielding,

$$
\begin{aligned}
\partial_{t} u_{\alpha}^{*} & +\partial_{\beta}\left\{\left(c_{s}^{2}+\frac{k}{\rho} \frac{\partial \phi}{\partial x_{\gamma}} \frac{\partial \phi}{\partial x_{\gamma}}\right) \delta_{\alpha \beta}-\frac{k}{\rho} \frac{\partial \phi}{\partial x_{\alpha}} \frac{\partial \phi}{\partial x_{\beta}}+u_{\alpha}^{*} u_{\beta}^{*}\right. \\
& \left.+c_{s}^{2}\left(\tau_{g}^{*}-\frac{1}{2}\right)\left(\frac{\partial u_{\alpha}}{\partial x_{\beta}}+\frac{\partial u_{\beta}}{\partial x_{\alpha}}\right) \Delta t\right\} \\
& -\partial_{\beta}\left\{c_{s}^{2} \Delta t\left(\tau_{g}^{*}-\frac{1}{2}\right)\left(\partial_{\alpha} u_{\beta}^{*}+\partial_{\beta} u_{\alpha}^{*}\right)\right\} \\
& +\partial_{\beta}\left\{\left(\tau_{g}^{*}-\frac{1}{2}\right) \partial_{\gamma}\left(u_{\alpha}^{*} u_{\beta}^{*} u_{\gamma}^{*}\right) \Delta t\right\} \\
& -\partial_{\alpha} \partial_{t_{0}}\left\{\left(\tau_{g}^{*}-\frac{1}{2}\right) \frac{k}{\rho}\left(\frac{\partial \phi}{\partial x_{\gamma}} \frac{\partial \phi}{\partial x_{\gamma}}\right) \Delta t\right\} \\
& +\partial_{\beta} \partial_{t_{0}}\left\{\left(\tau_{g}^{*}-\frac{1}{2}\right) \frac{k}{\rho}\left(\frac{\partial \phi}{\partial x_{\alpha}} \frac{\partial \phi}{\partial x_{\beta}}\right) \Delta t\right\} \\
& +\partial_{\beta} \partial_{t_{0}}\left\{c_{s}^{2}\left(\tau_{g}^{*}-\frac{1}{2}\right)^{2}\left(\frac{\partial u_{\alpha}}{\partial x_{\beta}}+\frac{\partial u_{\beta}}{\partial x_{\alpha}}\right) \Delta t^{2}\right\} \\
& +u_{\alpha}^{*} \partial_{\beta}^{2}\left\{\left(\tau_{g}^{*}-\frac{1}{2}\right) \frac{k}{\rho}\left(\frac{\partial \phi}{\partial x_{\gamma}} \frac{\partial \phi}{\partial x_{\gamma}}\right) \Delta t\right\} \\
& +u_{\alpha}^{*} \partial_{\beta} \partial_{\gamma}\left\{\left(\tau_{g}^{*}-\frac{1}{2}\right) \frac{k}{\rho}\left(\frac{\partial \phi}{\partial x_{\beta}} \frac{\partial \phi}{\partial x_{\gamma}}\right) \Delta t\right\} \\
& +u_{\beta}^{*} \partial_{\beta} \partial_{\gamma}\left\{c_{s}^{2}\left(\tau_{g}^{*}-\frac{1}{2}\right)^{2}\left(\frac{\partial u_{\gamma}}{\partial x_{\alpha}}+\frac{\partial u_{\alpha}}{\partial x_{\gamma}}\right) \Delta t^{2}\right\}=0 \\
+ & u_{\alpha}^{*} \partial_{\beta} \partial_{\gamma}\left\{c_{s}^{2}\left(\tau_{g}^{*}-\frac{1}{2}\right)\left(\frac{\partial u_{\gamma}}{\partial x_{\beta}}+\frac{\partial u_{\beta}}{\partial x_{\gamma}}\right) \Delta t^{2}\right\} \\
+ & \left.\left.u_{g}^{*}-\frac{1}{2}\right) \frac{k}{\rho}\left(\frac{\partial \phi}{\partial x_{\gamma}} \frac{\partial \phi}{\partial x_{\gamma}}\right) \Delta t\right\} \\
& \left.\left.+\frac{\partial}{\partial x_{\gamma}}\right) \Delta t\right\}
\end{aligned}
$$

In the latter equation, the terms including $\partial_{\beta}\left\{c_{s}^{2} \Delta t\left(\tau_{g}^{*}-\frac{1}{2}\right)\left(\partial_{\alpha} u_{\beta}^{*}+\partial_{\beta} u_{\alpha}^{*}\right)\right\}$ cancel each other out. The last term $\left(u_{\alpha}^{*} u_{\beta}^{*} u_{\gamma}^{*}\right)$ is $\mathcal{O}\left(\mathrm{Ma}^{3}\right)$, in Mach number and, hence, can be neglected. The terms $\partial_{\alpha} \partial_{t_{0}}\left\{\left(\tau_{g}^{*}-\frac{1}{2}\right) \frac{k}{\rho}\left(\frac{\partial \phi}{\partial x_{\gamma}} \frac{\partial \phi}{\partial x_{\gamma}}\right) \Delta t\right\}$, $\partial_{\beta} \partial_{t_{0}}\left\{\left(\tau_{g}^{*}-\frac{1}{2}\right) \frac{k}{\rho}\left(\frac{\partial \phi}{\partial x_{\alpha}} \frac{\partial \phi}{\partial x_{\beta}}\right) \Delta t\right\}, u_{\alpha}^{*} \partial_{\beta}^{2}\left\{\left(\tau_{g}^{*}-\frac{1}{2}\right) \frac{k}{\rho}\left(\frac{\partial \phi}{\partial x_{\gamma}} \frac{\partial \phi}{\partial x_{\gamma}}\right) \Delta t\right\}$, $u_{\alpha}^{*} \partial_{\beta} \partial_{\gamma}\left\{\left(\tau_{g}^{*}-\frac{1}{2}\right) \frac{k}{\rho}\left(\frac{\partial \phi}{\partial x_{\beta}} \frac{\partial \phi}{\partial x_{\gamma}}\right) \Delta t\right\}, \quad u_{\beta}^{*} \partial_{\beta} \partial_{\alpha}\left\{\left(\tau_{g}^{*}-\frac{1}{2}\right) \frac{k}{\rho}\left(\frac{\partial \phi}{\partial x_{\gamma}} \frac{\partial \phi}{\partial x_{\gamma}}\right) \Delta t\right\} \quad$ and $u_{\beta}^{*} \partial_{\beta} \partial_{\gamma}\left\{\left(\tau_{g}^{*}-\frac{1}{2}\right) \frac{k}{\rho}\left(\frac{\partial \phi}{\partial x_{\alpha}} \frac{\partial \phi}{\partial x_{\gamma}}\right) \Delta t\right\}$ contain the small parameter $k$ multiplying 
$\Delta t$ and thus can be neglected. Finally, $\partial_{\beta} \partial_{t_{0}}\left\{c_{s}^{2}\left(\tau_{g}^{*}-\frac{1}{2}\right)^{2}\left(\frac{\partial u_{\alpha}}{\partial x_{\beta}}+\frac{\partial u_{\beta}}{\partial x_{\alpha}}\right) \Delta t^{2}\right\}$, $u_{\alpha}^{*} \partial_{\beta} \partial_{\gamma}\left\{c_{s}^{2}\left(\tau_{g}^{*}-\frac{1}{2}\right)^{2}\left(\frac{\partial u_{\gamma}}{\partial x_{\beta}}+\frac{\partial u_{\beta}}{\partial x_{\gamma}}\right) \Delta t^{2}\right\}$ and $u_{\beta}^{*} \partial_{\beta} \partial_{\gamma}\left\{c_{s}^{2}\left(\tau_{g}^{*}-\frac{1}{2}\right)^{2}\left(\frac{\partial u_{\gamma}}{\partial x_{\alpha}}+\frac{\partial u_{\alpha}}{\partial x_{\gamma}}\right) \Delta t^{2}\right\}$ are on the order of $\Delta t^{2}$ and can be eliminated.

After accounting for the above simplifications, Eq. D.38 becomes,

$$
\partial_{t} u_{\alpha}^{*}+\partial_{\beta}\left(u_{\alpha}^{*} u_{\beta}^{*}\right)=\frac{k}{\rho} \partial_{\beta}\left\{\frac{\partial \phi}{\partial x_{\gamma}} \frac{\partial \phi}{\partial x_{\gamma}} \delta_{\alpha \beta}-\frac{\partial \phi}{\partial x_{\alpha}} \frac{\partial \phi}{\partial x_{\beta}}\right\}
$$

which is identical to Eq. (2.3) without viscous forces, gravitational force and pressure gradient. The effects of viscous and gravitational forces are added as body forces to the LB scheme as proposed in Guo et al. 2002. 


\section{APPENDIX E}

\section{Lattice Boltzmann method with non-uniform grid}

This section is based on the article "On the Finite Difference-Based Lattice Boltzmann Method in Curvilinear Coordinates, Renwei, Mei and Wei Shyy, Journal of Computational Physics,Volume 143, Issue 2, 1998, Pages 426-448"

Lattice Boltzmann Method is based on Boltzmann equation which had been derived in 1872 by Ludwig Boltzmann. The Boltzmann equation governs particle distribution function $f(\boldsymbol{x}, t, \boldsymbol{\xi})$, which specifies the probability to find a particle of fluid at position $\boldsymbol{x}$ at time t with particle velocity $\boldsymbol{\xi}$.

$$
\frac{\partial f(\boldsymbol{x}, t, \boldsymbol{\xi})}{\partial t}+\boldsymbol{\xi} \cdot \frac{\partial f(\boldsymbol{x}, t, \boldsymbol{\xi})}{\partial \boldsymbol{x}}=\Omega
$$

The left hand side of the Boltzmann equation is of advection type, while the right hand side contains the collision operator $\Omega$, which describes the interaction of particles.

For continuum flows with low Knudsen numbers, discretized velocities $\boldsymbol{e}_{i}$ may be introduced in order to obtain a model with reduced computational costs. A particle is only allowed to move in the limited number of directions and specific distances, so that Eq E.1 can be transformed to a set of discrete Boltzmann equations:

$$
\frac{\partial f_{i}(\boldsymbol{x}, t)}{\partial t}+\boldsymbol{e}_{i} \cdot \frac{\partial f_{i}(\boldsymbol{x}, t)}{\partial \boldsymbol{x}}=\Omega_{i}
$$

In this work the D3Q19 model is used for the discretization of the velocity 
space (Fig. 3.1). The discrete velocities $e_{i}$ for this model are $(i=0, \ldots, 18)$ :

$$
\boldsymbol{e}_{i}=c .\left\{\begin{array}{r|rrrrrr|rrrrrrrrrrrr}
0 & 1 & -1 & 0 & 0 & 0 & 0 & 1 & -1 & 1 & -1 & 1 & -1 & 1 & -1 & 0 & 0 & 0 & 0 \\
0 & 0 & 0 & 1 & -1 & 0 & 0 & 1 & -1 & -1 & 1 & 0 & 0 & 0 & 0 & 1 & -1 & 1 & -1 \\
0 & 0 & 0 & 0 & 0 & 1 & -1 & 0 & 0 & 0 & 0 & 1 & -1 & -1 & 1 & 1 & -1 & -1 & 1
\end{array}\right\}
$$

where $c$ is called particle speed in lattice. A finite difference discretization in space and time on a grid leads this equation to

$$
f_{i}^{n+1}=f_{i}^{n}-\Delta t e_{i} \cdot \nabla_{h} f_{i}^{n}+\Delta t \Omega_{i}
$$

For collision operator BGK single relaxation time (SRT)collision operator is used

$$
\Omega_{i}=\frac{1}{\tau}\left(f_{i}^{n}-f_{i}^{(e q) n}\right)
$$

where

$$
f_{i}^{(e q)}=w_{i}\left\{1+\rho_{0}\left(\frac{e_{i \alpha} u_{\alpha}}{c_{s}^{2}}+\frac{\left(e_{i \alpha} u_{\alpha}\right)^{2}}{2 c_{s}^{4}}-\frac{|u|^{2}}{2 c_{s}^{2}}\right)\right\}
$$

where $\tau$ is relaxation time and related to the fluid viscosity as

$$
\tau=3 \frac{\nu}{c^{2}}
$$

The velocity and pressure field are derived from representing momenta:

$$
\begin{aligned}
& u_{\alpha}=\frac{1}{\rho_{0}} \sum_{0}^{18} f_{i} e_{i \alpha} \\
& \rho=\sum_{0}^{18} f_{i}
\end{aligned}
$$




$$
p=c s^{2} \rho
$$

It can been shown with Champan-Enskog expansion that the solution of Lattice Boltzmann Method converges to the incompressible Navier-Stokes equation.

For the discretization of the spatial gradient.$\nabla$ for the general curvilinear coordinate $\boldsymbol{\xi}$, we can use procedure below :

$$
\boldsymbol{e}_{i} \cdot \nabla_{h} f_{i}=e_{i \alpha} \frac{\partial f_{i}}{\partial x_{\alpha}}=e_{i \alpha} \frac{\partial \xi_{\beta}}{\partial x_{\alpha}} \frac{\partial f_{i}}{\partial \xi_{\beta}}
$$

For one-dimensional stretching $(x=x(\xi), y=y(\eta)$ and $z=z \gamma)$ Eq. E.4 becomes

$$
f_{i}^{n+1}=f_{i}^{n}-\Delta t\left(\frac{e_{i x}}{h_{x}} \frac{\partial f_{i}}{\partial \xi}+\frac{e_{i y}}{h_{y}} \frac{\partial f_{i}}{\partial \eta}+\frac{e_{i z}}{h_{z}} \frac{\partial f_{i}}{\partial \gamma}-\Omega_{i}\right)
$$

where $h_{x}=d x / d \xi, h_{y}=d y / d \eta$ and $h_{z}=d z / d \gamma$.

\section{Finite difference calculation}

Central finite difference is easy to implement but for the flow with high Re number could produce artificial wiggles and make the simulation unstable. On the other hand the upwind FD often is stable but could lead to high numerical dissipation which could change the physics of the flow particularly for high Re number flows. Therefore for calculation of the gradient a mixed of central and upwind finite difference has been used by

$$
\frac{\partial f_{i}}{\partial \xi_{\beta}}=\left.\epsilon \frac{\partial f_{i}}{\partial \xi_{\beta}}\right|_{\text {central }}+\left.(1-\epsilon) \frac{\partial f_{i}}{\partial \xi_{\beta}}\right|_{\text {upwind }}
$$


where

$$
\left.\frac{\partial f_{i}}{\partial \xi_{\beta}}\right|_{c e n t r a l}=\frac{1}{2 \Delta \xi_{\beta}}\left[f_{i}\left(\xi_{\beta}+\Delta \xi_{\beta}, .\right)-f_{i}\left(\xi_{\beta}-\Delta \xi_{\beta}, .\right)\right]
$$

$$
\left.\frac{\partial f_{i}}{\partial \xi_{\beta}}\right|_{\text {upwind }}== \begin{cases}\frac{1}{2 \Delta \xi_{\beta}}\left[3 f_{i}\left(\xi_{\beta}+\Delta \xi_{\beta}, .\right)-4 f_{i}\left(\xi_{\beta}-\Delta \xi_{\beta}, .\right)+2 f_{i}\left(\xi_{\beta}-2 \Delta \xi_{\beta}, .\right)\right] & e_{i \beta \geq 0} \\ \frac{-1}{2 \Delta \xi_{\beta}}\left[3 f_{i}\left(\xi_{\beta}+\Delta \xi_{\beta}, .\right)-4 f_{i}\left(\xi_{\beta}+\Delta \xi_{\beta}, .\right)+2 f_{i}\left(\xi_{\beta}+2 \Delta \xi_{\beta}, .\right)\right] & e_{i \beta<0}\end{cases}
$$

\section{Validation}

In Fig. (E.1), two dimensional Poiseuille flow using non uniform grid has been shown and profiles of normalized velocity component are compared with analytical profile.

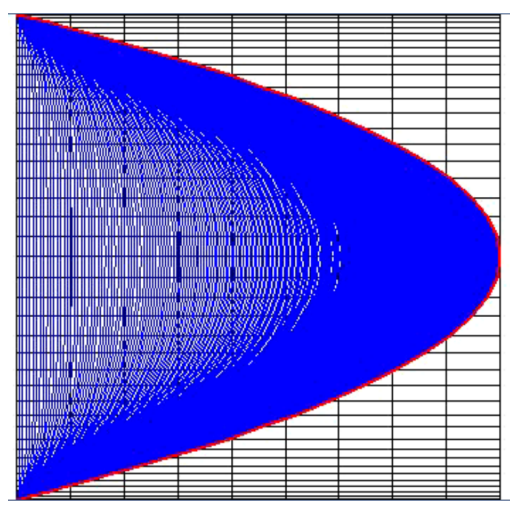

Figure E.1: Poiseuille flow,blue lines current result, red line, analytical profile

In Fig. E.2 an unsteady Couette flow velocity profile is shown. The velocity profile converges to linear profile as expected.

Cavity flow simulation results are shown in Fig. (E.3). In this simulation Reynolds number $R e=400$ based on lid velocity and cavity height. Profiles of normalized velocity component $u$ through the geometric center of the cavity at Re $=400$ is compared to result of Lee et al. 2001 . 

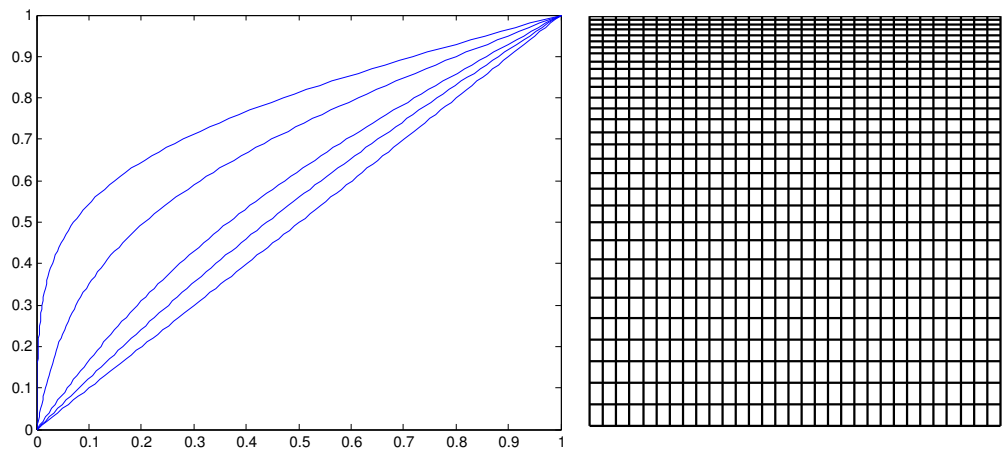

Figure E.2: Velocity profiles at different times of unsteady Couette flow and the computational mesh
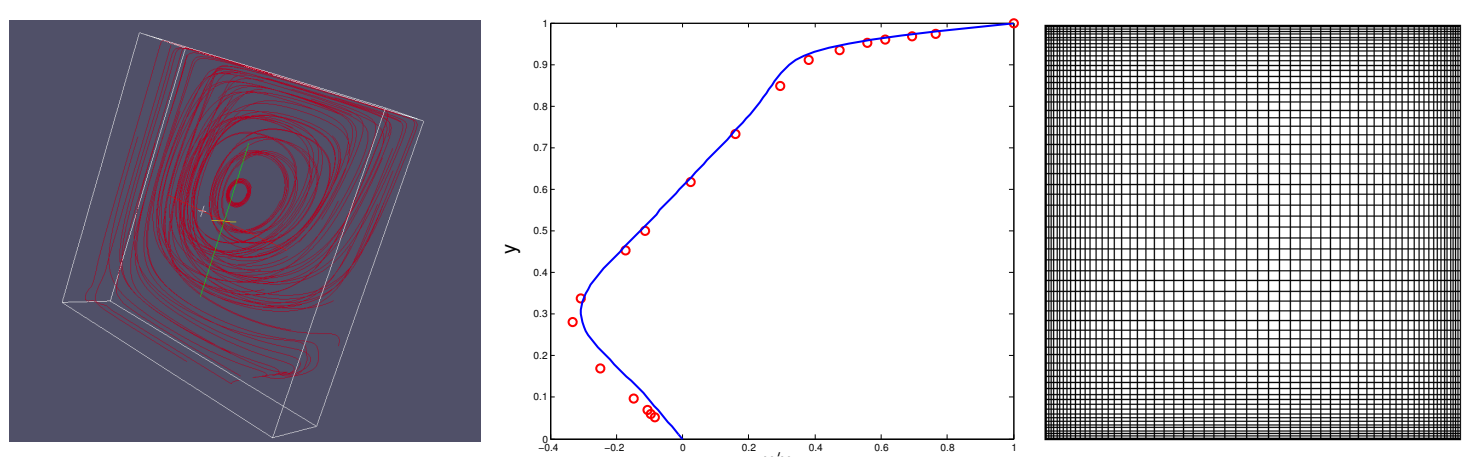

Figure E.3: Profiles of normalized velocity component $u$ through the geometric center of the cavity at $\operatorname{Re}=400$ circle-result of Lee et all 2001 blue line-current result 